\title{
Nuclear export of actin: \\ A biochemical and structural perspective
}

\author{
Dissertation \\ in partial fulfillment of the requirements \\ for the degree \\ "Doctor rerum naturalium (Dr. rer. nat)" \\ in the Molecular Biology Program \\ at the Georg-August University Göttingen, \\ Faculty of Biology
}

submitted by

Kevser Gençalp

born in

İzmir, Turkey

Göttingen, September 2014 


\section{Thesis Committee}

Prof. Dr. Dirk Görlich

(Supervisor)

Prof. Dr. Jörg Grosshans

Prof. Dr. Reinhard Jahn
Department of Cellular Logistics

Max Planck Institute for biophysical chemistry

Göttingen, Germany

Department of Developmental Biochemistry

Göttingen University Medical School

Göttingen, Germany

Department of Neurobiology

Max Planck Institute for biophysical chemistry

Göttingen, Germany

Members of the Examination Board

Prof. Dr. Dirk Görlich

Department of Cellular Logistics

(Referee)

Max Planck Institute for biophysical chemistry

Göttingen, Germany

Prof. Dr. Jörg Grosshans

Department of Developmental Biochemistry

( $2^{\text {nd }}$ Referee $)$

Göttingen University Medical School

Göttingen, Germany

\section{Further members of the Examination Board}

Prof. Dr. Peter Rehling

Prof. Dr. Markus Bohnsack

Prof. Dr. Holger Stark

Prof. Dr. Detlef Doenecke
Department of Cellular Biochemistry

Georg August University Göttingen

Göttingen, Germany

Department of Biochemistry I

Georg August University Göttingen

Göttingen, Germany

Research Group 3D Cryo-Microscopy

Max Planck Institute for biophysical chemistry

Göttingen, Germany

Universitätsmedizin Göttingen (UMG)

Georg August University

Göttingen, Germany 
I hereby declare that I completed my dissertation entitled "Nuclear export of actin: A biochemical and structural perspective" independently and with no other sources and aids than quoted.

This dissertation has not been submitted elsewhere for any academic award or qualification.

Kevser Gençalp

Göttingen, September 2014 
What is a scientist after all? It is a curious man looking through a keyhole, the keyhole of nature, trying to understand what's going on.

Jacques Yves Cousteau 


\section{Table of Contents}

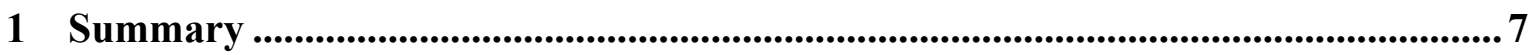

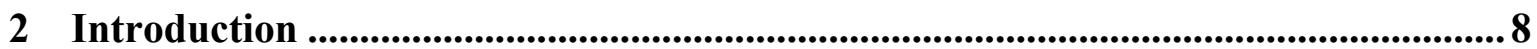

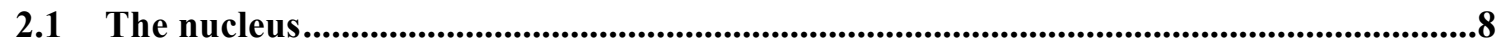

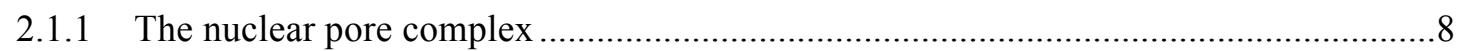

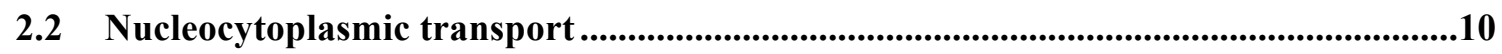

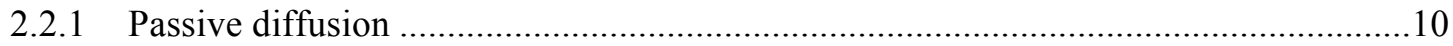

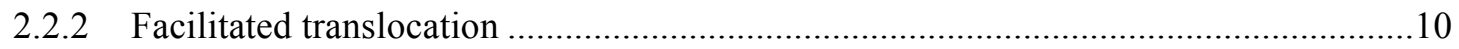

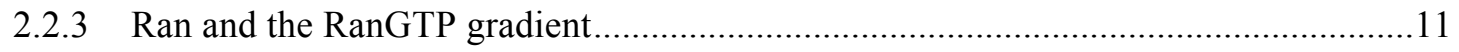

2.2.4 Nuclear import and export cycles..............................................................................

2.3 Nuclear Transport Receptors ..............................................................................................15

2.3.1 Structural features of $\operatorname{Imp} \beta$ family NTRs …….......................................................16

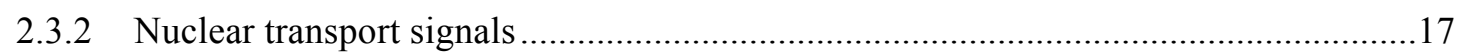

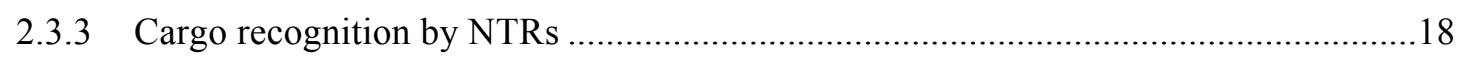

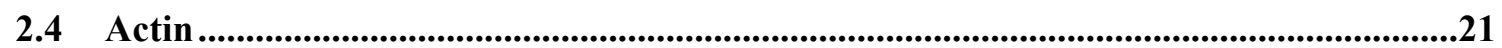

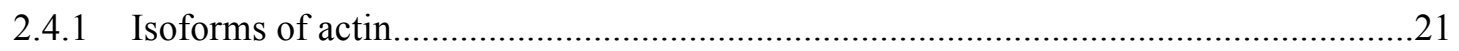

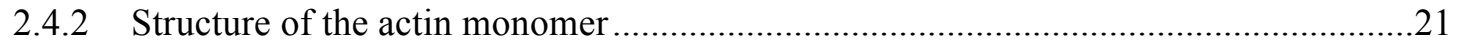

2.4.3 The actin filament and regulation of polymerization ...............................................22

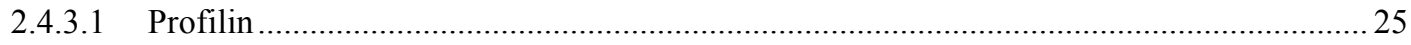

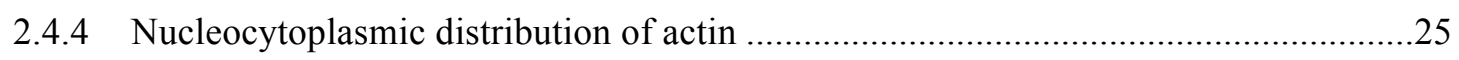

2.4.5 Exportin 6-mediated actin nuclear export pathway.................................................27

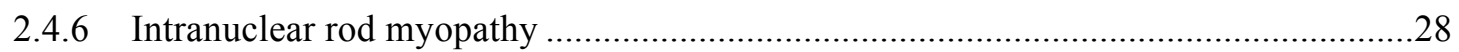

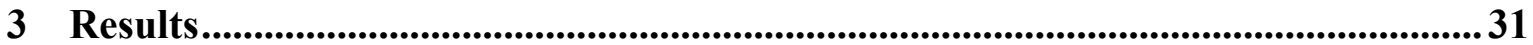

3.1 The actin isoforms differ in their affinity for the complex ............................................31

3.1.1 First attempts with $\alpha$-actin from skeletal muscle .......................................................31

3.1.2 Non-muscle actins form a more stable complex with profilin .....................................33

3.2 Screening for optimal conditions for the actin nuclear export complex .........................36

3.3 Xpo6 and RanGTP can form a stable dimeric complex in vitro......................................37

3.4 Actin export complex can be formed via two stable sub-complexes .................................38

3.5 Actin nuclear export complex is sensitive to ionic strength ..........................................40

3.5.1 The ionic species in the solution affect the export complex formation .......................42

3.6 Improving the strategy for the actin nuclear export complex formation.......................45

3.7 Topological analysis of the actin nuclear export complex ...................................................48

3.8 Thermal stability analysis of Exportin 6 and its complexes .............................................50

3.9 Crystallization of Exportin 6 and its complexes ............................................................52 


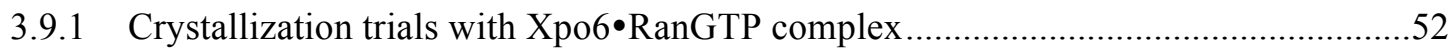

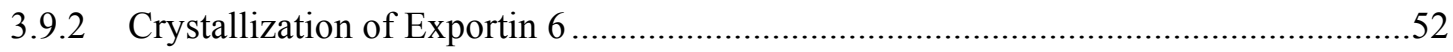

3.9.2.1 Limited proteolysis and secondary structure prediction of Xpo6 ……………………......59

3.9.2.2 Sequence conservation of Xpo6 in evolutionary distant species....................................... 64

3.9.3 Crystallization of the actin nuclear export complex.....................................................67

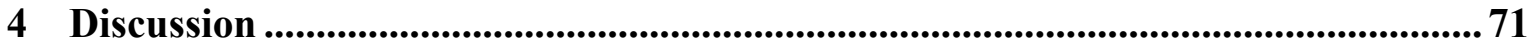

4.1 Actin isoforms and the complex stability …….....................................................................71

4.2 Stable Xpo6• RanGTP interaction in the absence of the cargo .........................................72

4.3 Crystallization of human Xpo6 ..........................................................................................72

4.4 Xpo6 in evolutionary distant species..................................................................................75

4.5 Ionic sensitivity of the actin nuclear export complex ...................................................75

4.6 Phenyl sepharose stabilizes the actin nuclear export complex ..........................................76

4.7 Topological analysis and crystallization of the actin nuclear export complex...............77

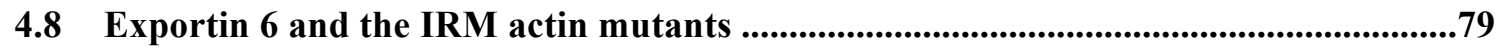

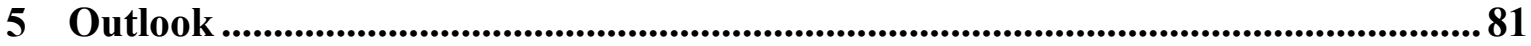

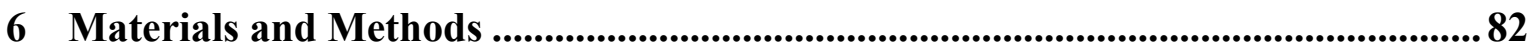

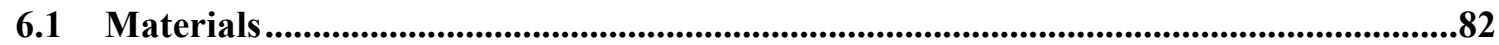

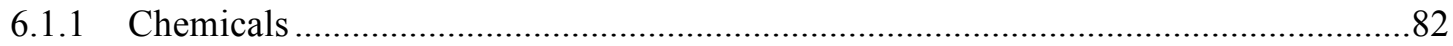

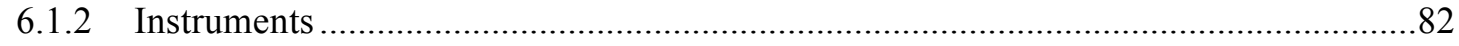

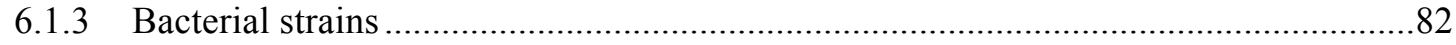

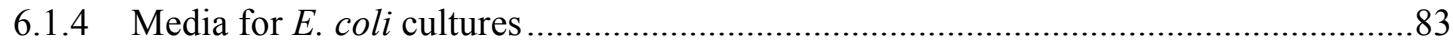

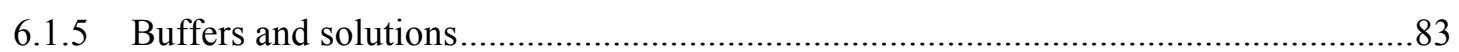

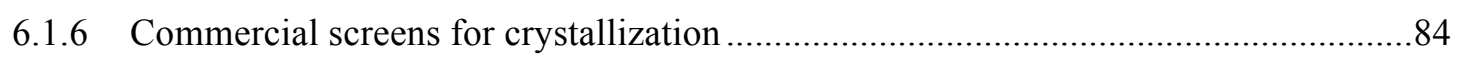

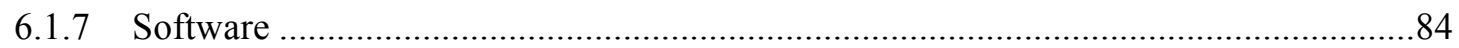

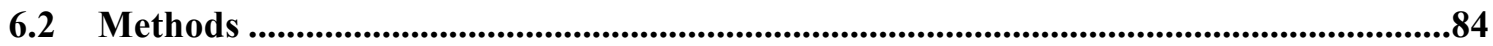

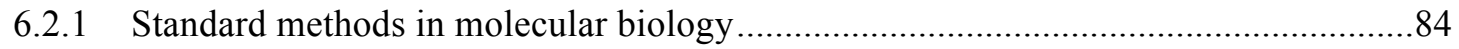

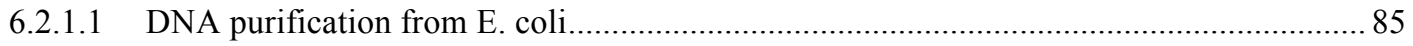

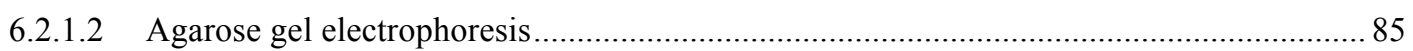

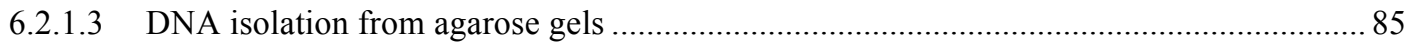

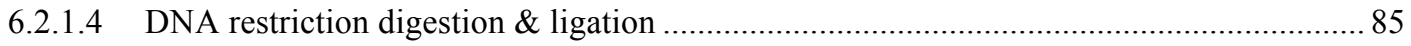

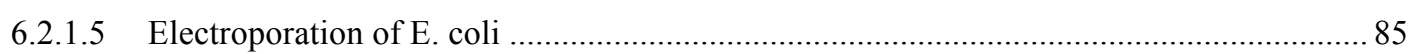

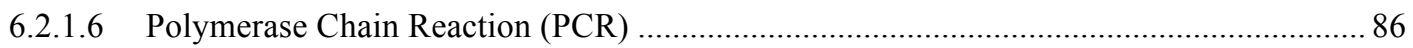

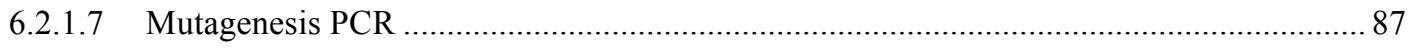

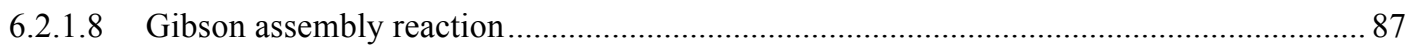

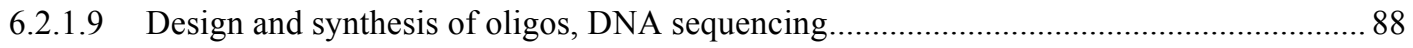

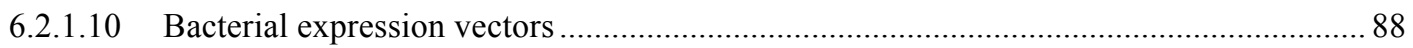

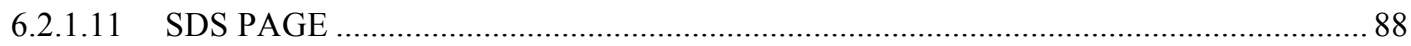




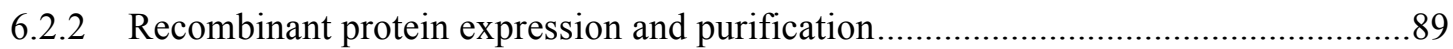

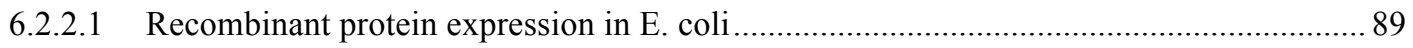

6.2.2.2 Native protein purification with $\mathrm{Ni}^{2+}$ affinity chromatography ....................................... 90

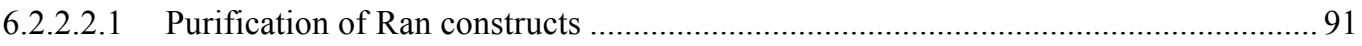

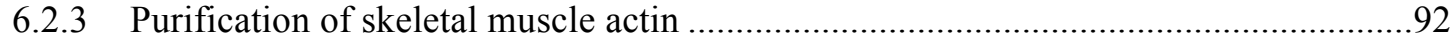

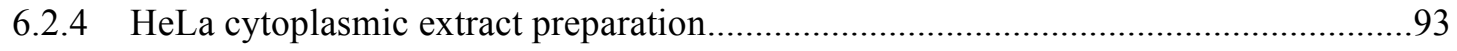

6.2.5 DNaseI coupling to cyanogen bromide activated sepharose.....................................94

6.2.6 Binding assays for complex formation.....................................................................94

6.2.7 Actin export complex formation for crystallization ....................................................95

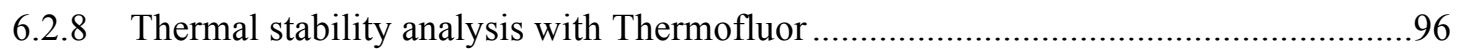

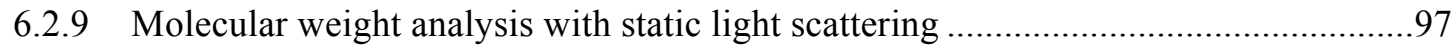

6.2.10 Polydispersity analysis by dynamic light scattering ….............................................97

6.2.11 Limited proteolysis of crystallization substrates .......................................................97

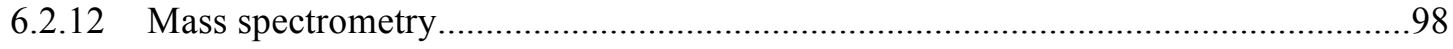

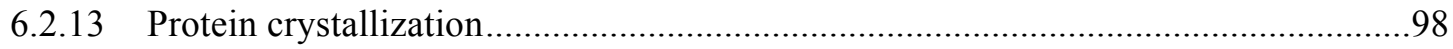

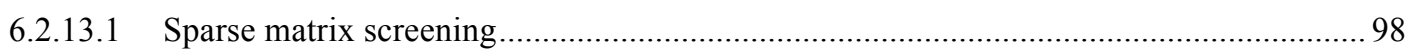

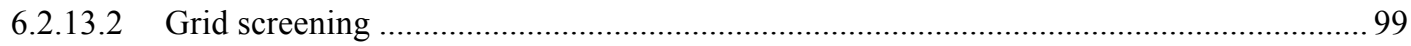

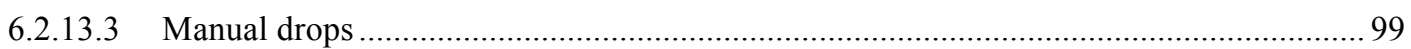

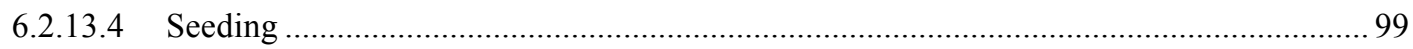

6.2.13.5 Cryoprotection, crystal fishing and data collection ..................................................... 100

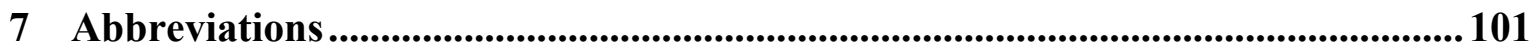

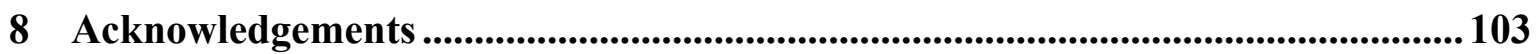

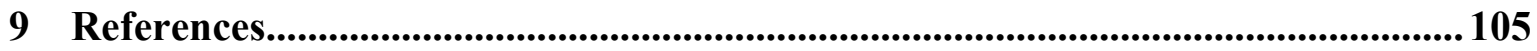

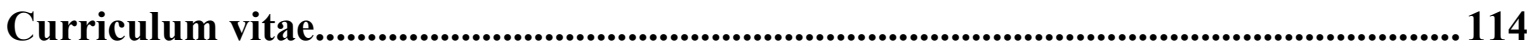




\section{List of Figures}

Figure 2-1 Nucleus, nuclear envelope and the nuclear pore complex...........................................9

Figure 2-2 Conformational changes in Ran upon nucleotide exchange ............................................12

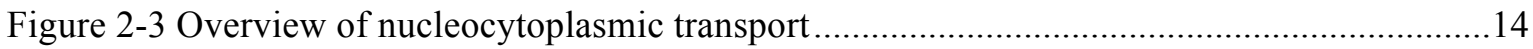

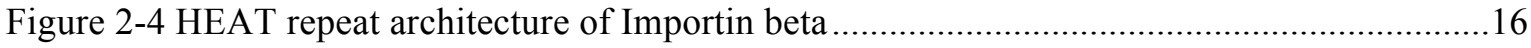

Figure 2-5 Comparison of structurally characterized exportins in cytoplasmic and nuclear states ..20

Figure 2-6 Structure of G-actin, profilactin and the actin filament ...................................................23

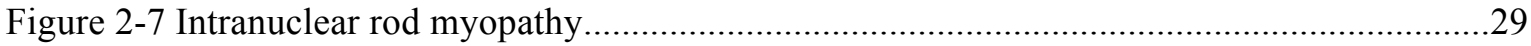

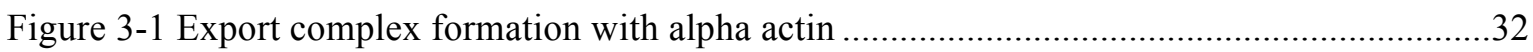

Figure 3-2 Profilactin with muscle and non-muscle actin isoforms.................................................34

Figure 3-3 A stable profilactin complex can be formed with cytoplasmic actin ..............................35

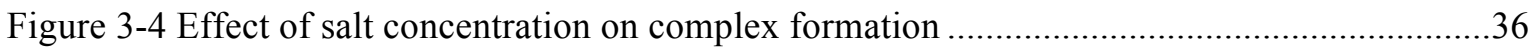

Figure 3-5 Effect of buffer ions and $\mathrm{pH}$ on complex formation ....................................................

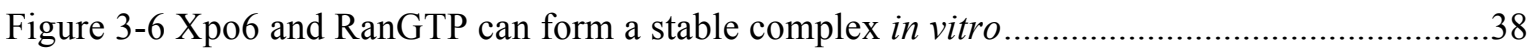

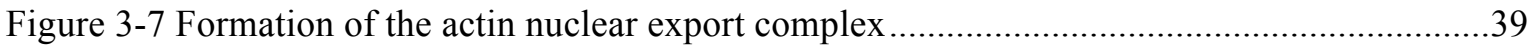

Figure 3-8 Increasing salt concentration dissociates a pre-formed actin export complex .................41

Figure 3-9 Formation of the actin nuclear export complex is salt sensitive......................................42

Figure 3-10 The ionic species in the buffer have an effect on the complex formation......................43

Figure $3-11 \mathrm{pH}$ and buffer ions have an effect on complex formation .............................................4

Figure 3-12 Purification of the actin nuclear export complex in large scale for crystallization ........46

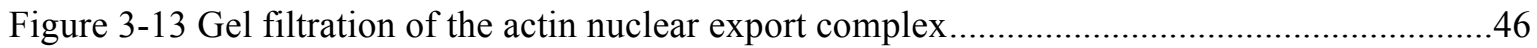

Figure 3-14 Molecular weight analysis of the actin nuclear export complex with MALS ...............47

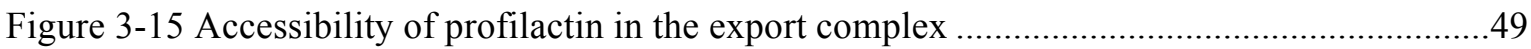

Figure 3-16 DNaseI can dissociate a pre-formed actin nuclear export complex .............................50

Figure 3-17 Themofluor measurements for Xpo6, Xpo6•RanGTP and the export complex ...........51

Figure 3-18 Melting curves for Xpo6, Xpo6•RanGTP and the export complex ……......................51

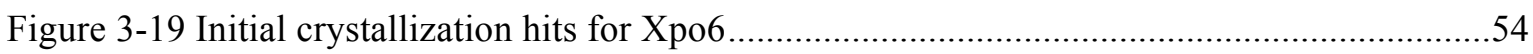

Figure 3-20 Xpo6 crystals from the refinement of "MBclass E8" ....................................................56

Figure 3-21 Xpo6 crystals from refinement of "Midas G1" and "MBclassE12" ...............................57

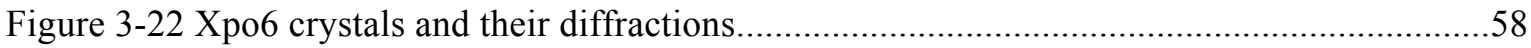

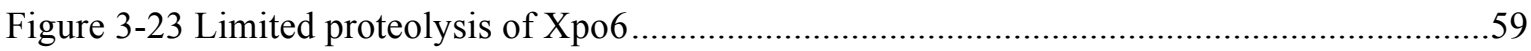

Figure 3-24 Secondary structure prediction for Xpo6 with "psipred" and identified protease

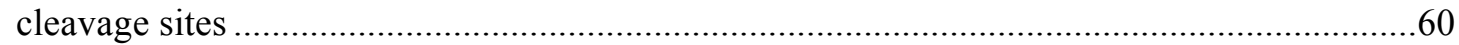

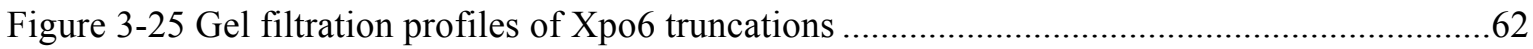

Figure 3-26 Actin export complex formation with Xpo6 truncations..............................................63

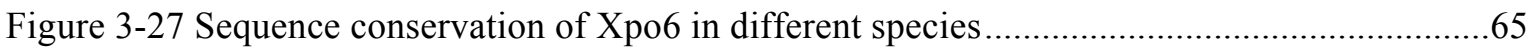




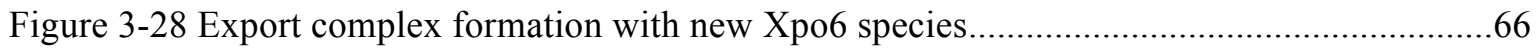

Figure 3-29 Initial hits for actin nuclear export complex crystallization ...........................................67

Figure 3-30 Reproduction of the actin nuclear export complex crystals ..........................................69

Figure 3-31 SDS PAGE analysis of the actin nuclear export complex crystals................................70

Figure 4-1 Mapping of the binding sites on the actin monomer ...................................................... 


\section{List of Tables}

Table 2-1 Mammalian nuclear transport receptors and selected cargos ..........................................15

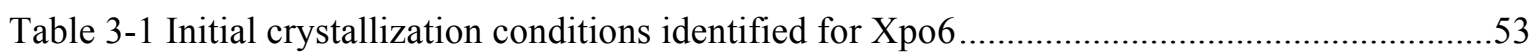

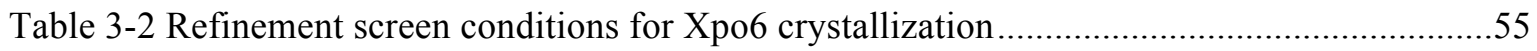

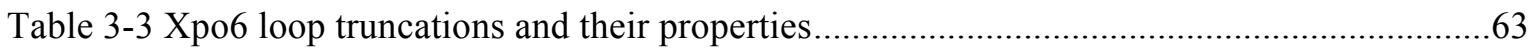

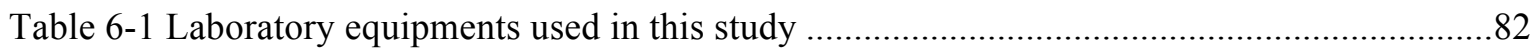

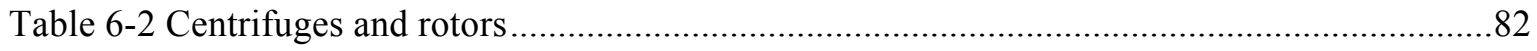

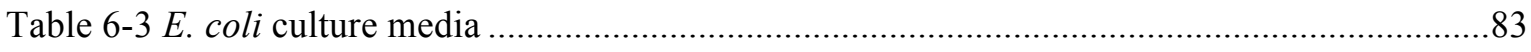

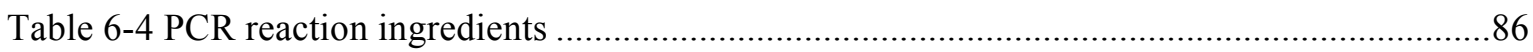

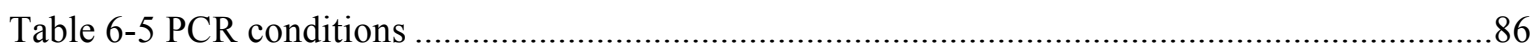

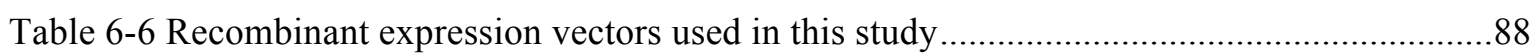

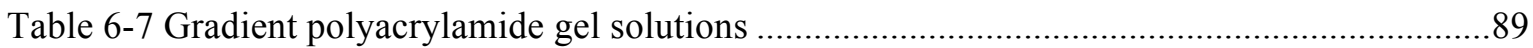




\section{Summary}

Macromolecular exchange between the nucleus and the cytoplasm is mainly mediated by the RanGTP-dependent nuclear transport receptors (NTRs) of the $\operatorname{Imp} \beta$ family. NTRs are classified as importins and exportins according to the direction of transport. Exportins carry their cargos from the nucleus to cytoplasm as a ternary export complex together with RanGTP. Imp $\beta$-like NTRs are made up of multiple HEAT repeats and share an inherently flexible structure. Nevertheless, structural analyses so far revealed unique cargo recognition mechanisms for each NTR, and even for different cargos recognized by the same receptor.

Actin is the well-known constituent of the cytoplasmic microfilaments. Monomeric actin with its small size and globular fold can slowly diffuse into the nucleus. Exportin 6 (Xpo6) is a dedicated export receptor found in vertebrates and insects, whose only known function is to export nuclear actin•profilin back to cytoplasm. In the absence of Xpo6, actin accumulates in the nucleus. Amphibian oocytes exploit this to stabilize their giant nuclei with an intranuclear actin cytoskeleton by blocking Xpo6 expression. On the other hand, nuclear accumulation of actin is also seen in intranuclear rod myopathies (IRM), devastating muscle diseases caused by several mutations of the skeletal alpha actin gene. We hypothesize that these mutations of actin interfere with its recognition and nuclear export by Xpo6, resulting in the characteristic nuclear accumulations.

To decipher the cargo recognition by Xpo6 and to understand the mechanisms underlying the IRM, we set out to crystallize the Xpo6•RanGTP•actin•profilin complex. It turned out that the actin export complex is extremely salt sensitive, and muscle and non-muscle actin isoforms differ in their affinity for profilin and hence, for the export complex. We developed a new single-step protocol for purification of profilin $\bullet \beta / \gamma$-actin complexes from cytoplasmic extracts, which enabled us to purify a stable actin export complex. Topological analysis of the actin export complex showed that the Poly-Proline-binding pocket of profilin is accessible in the complex, whereas the binding site for DNaseI on actin overlaps with the Xpo6 binding site. Crystals of cargo-free Xpo6 were obtained in several different conditions. However, the diffraction is currently limited to $7.4 \AA$ resolution. Also the actin nuclear export complex was successfully crystallized. These crystals will be the substrates for future structural analyses and will help us to understand the cargo recognition by Xpo6. 


\section{Introduction}

\subsection{The nucleus}

One of the key "inventions" in evolution is the organization of eukaryotic cells into membrane bound compartments. Confining certain processes to specific compartments allows the cell to carry out counteracting reactions simultaneously, and complementary reactions sequentially. More importantly, compartmentalization equips the cell with a tighter control over these processes. Advantages of compartmentalization can be best understood by the fact that only eukaryotes developed into complex multicellular organisms.

Nucleus is the defining compartment of a eukaryotic cell. It encloses the genome; separates DNA replication, DNA repair, transcription and mRNA processing (splicing of introns, polyadenylation, capping) events from the cytoplasmic translation, spatially and temporally. Nuclear mRNAs are only exported into the cytoplasm after being processed, which ensures that only the processed mRNAs are translated. This allows a level of control that makes intron-bearing genes possible. Intron-containing genes and alternative splicing provide increasing complexity to eukaryotes on functional level, without increasing the genome size proportionally. Also, containment of the genome in a specialized organelle protects it from mechanical and metabolic damage, and pathogens, increasing the genomic stability and enabling eukaryotes to handle larger genomes.

\subsubsection{The nuclear pore complex}

Nuclear envelope (NE) is a double membrane structure that defines the borders of the nucleus. The inner membrane of the NE faces the nuclear interior, whereas the outer membrane is continuous with the endoplasmic reticulum (ER) and faces cytoplasm. The space between the inner and the outer nuclear membranes (perinuclear space, PNS) is as well continuous with the ER lumen (Watson, 1955; Subramanian and Meyer, 1997). The nuclear envelope is punctured with disc-shaped openings, the nuclear pores, where the otherwise parallel outer and inner membranes of the NE meet (Watson, 1954). Nuclear pores are decorated with gigantic proteinaceous assemblies with octagonal symmetry, called nuclear pore complexes (NPCs) (Watson, 1959). NPCs are the main routes for nucleocytoplasmic exchange in the cell (Feldherr, 1962). 


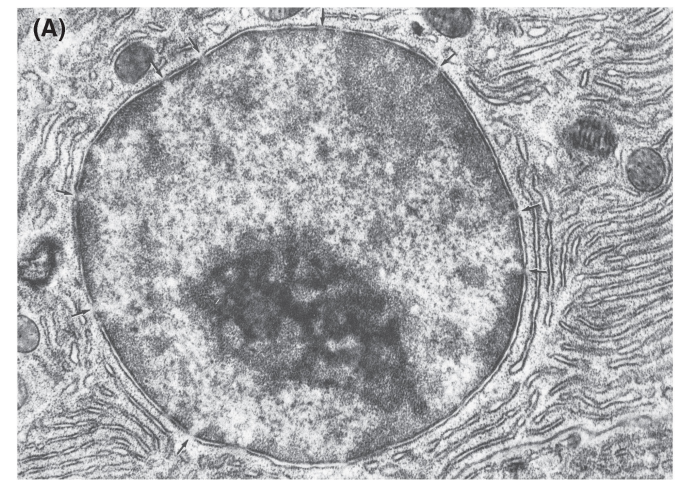

(C)

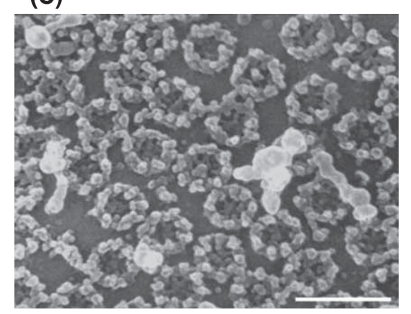

(D)

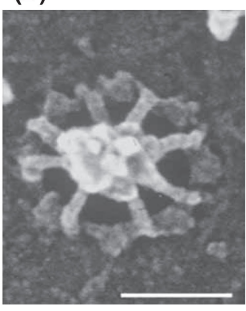

(B)

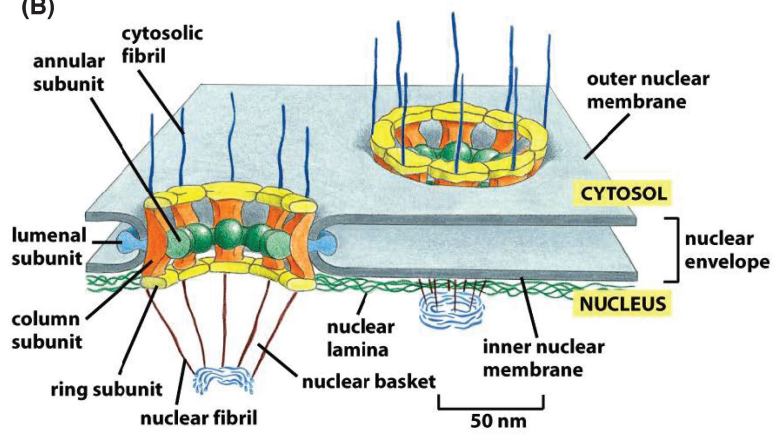

(E)

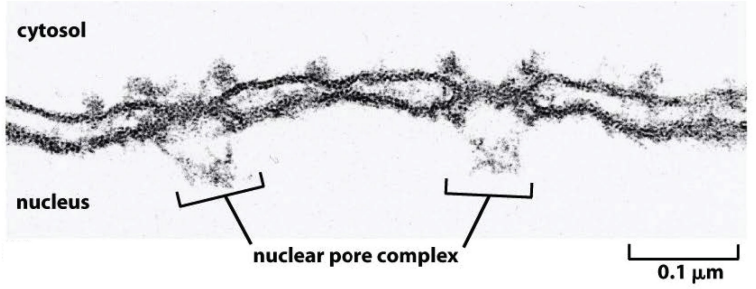

Figure 2-1 Nucleus, nuclear envelope and the nuclear pore complex

(A) Electron micrograph of pancreatic acinar cell nucleus from the bat Myotis lucifugus. (Fawcett, 1981). (B) Schematic representation of the nuclear membrane and NPCs (Alberts et al., 2007). Nuclear pore complexes have octagonal symmetry, which is easily recognized by observing the cytoplasmic fibrils and the nuclear basket (C) Cytoplasmic face of Xenopus laevis nuclear envelope and the nuclear pore complexes. Scale bar 200nm (Allen et al., 2007). (D) Nuclear basket of a single NPC from Xenopus laevis nuclear envelope. Scale bar $50 \mathrm{~nm}$ (Allen et al., 2007). (E) Side view of the nuclear envelope perforated with NPCs (Alberts et al., 2007).

The NPC is one of the largest complexes found in the cell with molecular weight reaching $\approx 66$ MDa for yeast NPCs (Rout and Blobel, 1993; Yang et al., 1998) and $\approx 125$ MDa for vertebrate NPCs (Reichelt et al., 1990). Despite its large size, the NPC is made up of only 30 different proteins, nucleoporins (Nup), which are present in multiple copies in the complex (see (Ori et al., 2013) for exact stoichiometries). Nups can be classified according to their location within the NPCs. Transmembrane Nups anchor to the NE and provide base for the channel to be build on. Scaffold Nups form the ring-like structure that surrounds the periphery of the central channel. Another class of Nups have dispersed phenylalanine-glycine (FG) repeats and these FG domains lack a defined fold (Denning et al., 2002, 2003). The FG-Nups bind to the scaffold and plug the center of the channel via their FG domains, fulfilling a barrier function. The structural organization of the NPC proteins within the pore and the principles of the NPC permeability barrier are not yet fully resolved. One of the best models to explain the NPC permeability barrier is the "selective phase" model (Ribbeck and Gorlich, 2001). The model states that the FG-Nups form a meshwork through inter- and intramolecular interactions via the FG repeats, which forms an aqueous passive diffusion barrier. This meshwork excludes inert molecules larger than the passive diffusion limit $(5 \mathrm{~nm})$, whereas molecules smaller than this limit can diffuse 
through this sieve-like structure. Molecules larger than the passive diffusion limit require assistance of specialized soluble receptors for nuclear transport, which can dissolve their way through this meshwork by interacting with the FG repeats.

\subsection{Nucleocytoplasmic transport}

The separation of the cytoplasm and the nucleus comes at the cost of active and selective transport between the two compartments. Translation being a solely cytoplasmic process, all the nuclear proteins that are needed for DNA stability, replication and repair, proteins necessary for transcription, RNA processing and gene expression regulation have to be imported from the cytoplasm. On the other hand, translation strictly depends on nuclear products such as the mRNAs, tRNAs, and the ribosomal subunits, which have to be exported from the nucleus.

Nucleocytoplasmic transport is an essential activity for the cell. In order to keep the cytoplasmic and nuclear contents separated and to move the necessary macromolecules across the NPC, the cell invests considerable amounts of energy and dedicated proteins. The following calculations give an impression about the extent of the energetic investment: a proliferating HeLa cell uses a nuclear transport capacity of $\sim 20 \mathrm{MDa}$ per NPC per second, which approximates to a total mass flow of $>200$ GDa per second between nucleus and cytoplasm (Ribbeck and Gorlich, 2001). Both passive diffusion and facilitated transport contribute to the material exchange through the NPC.

\subsubsection{Passive diffusion}

Small molecules and proteins up to $5 \mathrm{~nm}$ in diameter $(20-40 \mathrm{kDa})$ can freely diffuse through the NPC permeability barrier, whereas larger proteins require assistance for efficient translocation (Mohr et al., 2009). Passive diffusion does not require specific interactions between the translocating species and the NPC. Small proteins that are equally distributed between cytoplasm and nucleus and almost all metabolites and ions passively diffuse through the NPC.

\subsubsection{Facilitated translocation}

Most of the molecules that cross the NPC in either direction are indeed too large to be let through by the permeability barrier. Such molecules traverse the NPC with the aid of specialized soluble receptors. The nuclear transport receptors (NTRs) of the Imp $\beta$ family have the ability to dissolve into the FG meshwork of the NPC, explaining the rapid 
translocation rates of the NTRs compared to passive diffusion events (Ribbeck and Gorlich, 2001).

A facilitated active transport, however, is not only required for the large molecules. Proteins with regulatory function, including the transcription factors, are needed in the nucleus only at certain times. Obviously, passive diffusion for such molecules can not provide the necessary strictness. Like only the processed mRNAs being exported from the nucleus, these molecules can interact with their corresponding NTRs only when they represent the required properties. Regulating the NPC passage of such molecules by NTRs is an elegant solution to the problem.

NPC forms a tight, yet imperfect barrier. Even proteins that are slightly larger than the diffusion limit may slowly cross the NPC. This becomes more prominent, when long time scales are considered for the passive influx. For this reason, the lack of an active import mechanism does not guarantee the exclusion of a protein from nucleus. Specific nuclear exclusion requires an active export mechanism, which can work against a free concentration gradient, unlike the passive diffusion.

For example, the major cytoskeletal protein actin is $42 \mathrm{kDa}$ in its monomeric form and can slowly diffuse into the nucleus (De Robertis et al., 1978). Since actin can reach cytoplasmic concentrations of about $200 \mu \mathrm{M}$ (Alberts et al., 2002), it is essential that the leakage is counteracted by active transport. Exportin 6 is a specialized NTR, whose only function is to pump actin back into the cytoplasm (Stuven et al., 2003). The importance of the active transport becomes evident, when the polymerization capability of actin is considered. In the absence of the actin binding proteins to keep it monomeric, actin can quickly polymerize in the nucleus and interfere with the nuclear processes.

\subsubsection{Ran and the RanGTP gradient}

A $25 \mathrm{kDa}$ GTP binding protein, Ran (Ras-related nuclear antigen) (Bischoff and Ponstingl, 1991a), provides directionality to the active nucleocytoplasmic transport (Gorlich et al., 1996b). Ran can be found in two distinct nucleotide bound states, RanGTP and RanGDP (Gorlich et al., 1996b). Upon the hydrolysis of the gamma phosphate, the core of the protein (G-domain) remains mostly unchanged, whereas three regions, called switches, assume drastically different conformations. For details of the conformational changes of Ran, see Figure 2-2. These conformational changes make the GTP and GDP states of Ran functionally distinct. RanGTP (active form) binds to NTRs with high affinity, where 
RanGDP (inactive form) does not. The directionality of the active nuclear transport is determined by the differential localization of Ran species on either side of the NE. In the nucleus, RanGTP concentration is approximately 1000 fold higher than the cytoplasm (Gorlich et al., 2003). This steep concentration gradient is the driving force of facilitated translocation (Gorlich et al., 1996b).

RanGDP

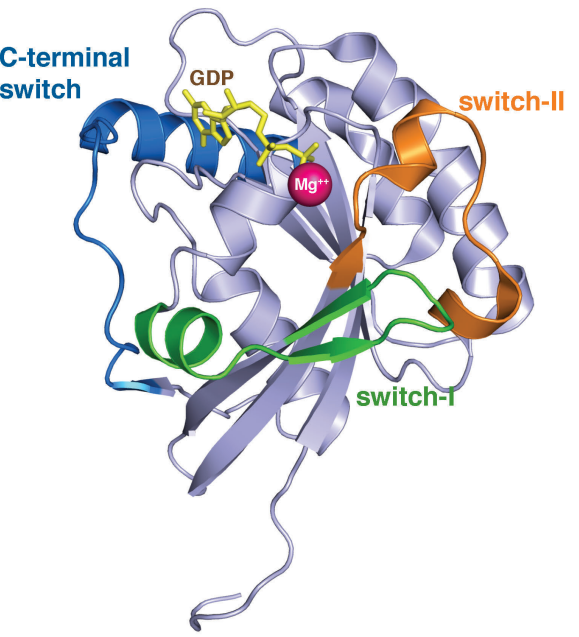

RanGTP

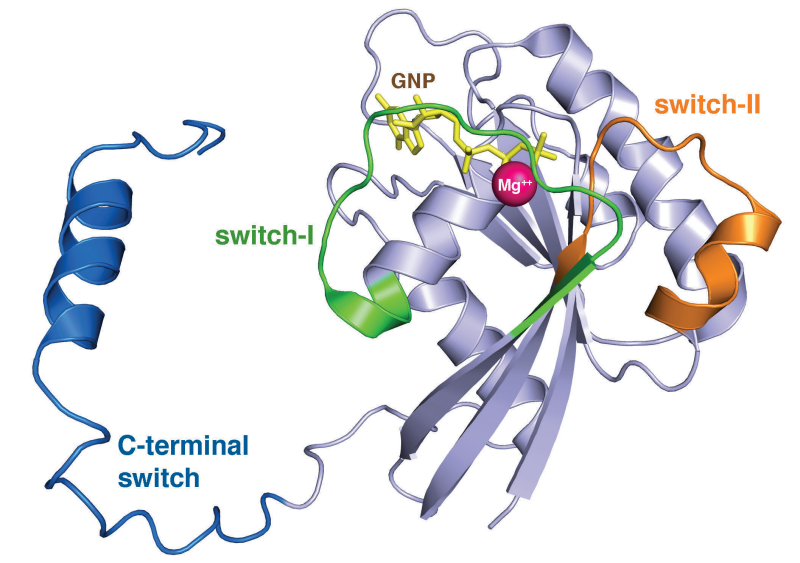

Figure 2-2 Conformational changes in Ran upon nucleotide exchange

Ran is shown in GDP and GTP bound states. For emphasis on the conformational changes, three main switch regions are colored in green (switch I, residues 30-47), in orange (switch II, residues 65-80) and in blue (C-terminal switch, residues 177-216). In the GDP bound state, Ran represents a more compact folding, with the C-terminal switch folded back onto the protein core. At the extreme C-terminus of Ran an acidic DEDDDL motif, which is not resolved in the crystal structure, is most likely to fold on a basic patch on the protein core. The switches I and II do not contact the nucleotide. Upon nucleotide exchange, major rearrangements occur in the molecule. Switch I undergoes a drastic conformational change, now making extensive contacts with the nucleotide. The changes in switch II are more subtle but no less important. Switch II bears the Gln 69 residue, which is essential for GTP hydrolysis, and with this subtle change in switch II, it is brought to close proximity to the $\gamma$ phosphate of GTP. C-terminal switch is now in an extended conformation and does not contact the core. The basic patch, which becomes free in the GTP conformation, is important for contact with NTRs. The conformation of the C-terminal switch will depend on the binding partner, which in this case is RanBP1. Ran GTP is taken from the structure with PDB-ID: 1K5D (Seewald et al., 2002). RanGDP is taken from crystal structure with PDB-ID: 3GJ0 (Partridge and Schwartz, 2009). The representation was prepared using PyMol.

Several proteins are involved in creating and maintaining this gradient. The intrinsic GTPase activity of Ran is very low, such that the GTP hydrolysis strictly depends on the Ran GTPase activating protein RanGAP (Bischoff et al., 1994; Klebe et al., 1995). RanGAP, however, can not act on a RanGTP molecule that is bound to an NTR. For the removal of Ran from the NTR, additional proteins are required. RanBP1 or RanBP2 (Nup358) help to destabilize the NTR·RanGTP complexes and the eventual hydrolysis of GTP together with RanGAP (Bischoff and Gorlich, 1997). RanBP2 is a part of the NPC on the cytoplasmic side (Yokoyama et al., 1995), whereas RanBP1 and RanGAP are kept exclusively cytoplasmic (Matunis et al., 1996; Richards et al., 1996; Mahajan et al., 1997). 
It is important that all these proteins are confined to the cytoplasm, to ensure that the GTP hydrolysis only takes place in this compartment. A fraction of soluble RanGAP is SUMOylated and is recruited to the cytoplasmic side of NPC via RanBP2 (Matunis et al., 1996). This localization of RanGAP not only serves a rapid recycling of the NTRs, but also ensures that the steep RanGTP gradient is maintained across the NE.

On the nuclear side of the NE, another protein acts in the opposite way: generation of RanGTP. The chromatin bound guanine nucleotide exchange factor of Ran, RCC1 (regulator of chromosome condensation 1 ) catalyzes the exchange of Ran bound GDP to GTP, and constantly replenishes the nuclear RanGTP levels (Bischoff and Ponstingl, 1991b). It is essential to confine the regulatory proteins to their respective compartments, in order to create and maintain the RanGTP gradient across the NE (Izaurralde et al., 1997).

\subsubsection{Nuclear import and export cycles}

NTRs shuttle between the cytoplasm and the nucleus, bind to their cargos via specific signals, carry them through the NPC, and release them on the destination site (Gorlich and Kutay, 1999). NTRs are classified according to their transport directions: importins (Gorlich et al., 1994) bind their cargos in the cytoplasm and release them in the nucleus; and exportins (Fornerod et al., 1997) bind their cargos in the nucleus and release them in the cytoplasm. A more detailed analysis of the NTRs of Imp $\beta$ superfamily will be presented in the section 2.3. Importins bind their cargos in the cytoplasm, where RanGTP concentration is low. Upon translocation to the nucleus, RanGTP dissociates the importin॰cargo complex. Cargo is released and the RanGTP bound importin shuttles back to the cytoplasm. In the cytoplasm, RanGTP is dissociated from the importin making it available for another round of transport. Exportins, on the other hand, bind their cargos in the nucleus together with RanGTP. There is cooperativity in cargo and RanGTP binding, such that binding of RanGTP increases the affinity towards the cargo and vice versa. The ternary export complex translocates through the NPC, and is dissociated upon reaching the cytoplasm. Cargo and Ran are released, and the free exportin shuttles back to nucleus for another round.

As described, both import and export cycles result in the removal of one RanGTP from the nucleus per cycle. A small transporter, Nuclear Transport Factor 2 (NTF2), shuttles Ran back to the nucleus counteracts this constant RanGTP drain. NTF2 is a $15 \mathrm{kDa}$ protein that 
works as a homodimer and imports two RanGDP molecules to the nucleus (Moore and Blobel, 1994; Ribbeck et al., 1998). In the nucleus RanGDP is converted to RanGTP by $\mathrm{RCC} 1$ and is released from the NTF2. Figure 2-3 gives an overview of nuclear import and export cycles. The GTP hydrolysis by Ran is the only form of energetic input to the nuclear transport

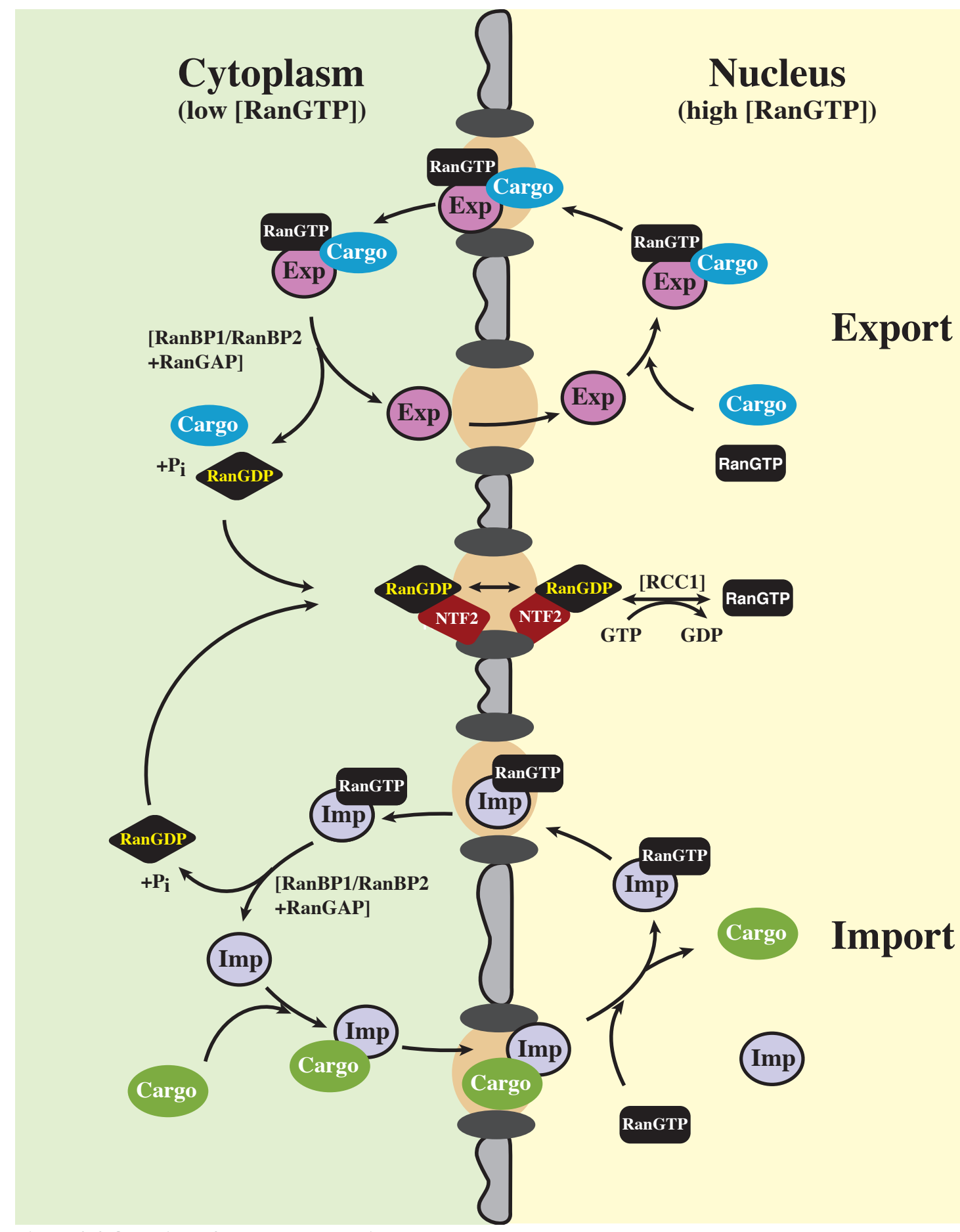

Figure 2-3 Overview of nucleocytoplasmic transport

The scheme summarizes the nuclear import, export and Ran cycles. Importins are abbreviated as Imp, exportins as Exp. The figure was adopted and modified from (Gorlich and Kutay, 1999). 


\subsection{Nuclear Transport Receptors}

To date, there are more than 20 NTRs of the Importin $\beta$ superfamily known in mammals. While most NTRs are dedicated to either import or export, there are also NTRs shown to work in both directions with different cargos. Table 2-1 shows a list of functionally characterized mammalian NTRs and a selection of their respective cargos.

Table 2-1 Mammalian nuclear transport receptors and selected cargos

\begin{tabular}{|c|c|c|}
\hline NTR & Selected cargos & References \\
\hline \multicolumn{3}{|l|}{ Export receptors } \\
\hline Exportin 1 (CRM1) & $\begin{array}{l}\text { Leu-rich NES cargos } \\
\text { HIV-Rev } \\
\text { Snurportin } 1 \\
\text { 60S ribosomal subunit } \bullet \text { Nmd3 }\end{array}$ & $\begin{array}{l}\text { (Wen et al., 1995) } \\
\text { (Fischer et al., 1995) } \\
\text { (Fornerod et al., 1997) } \\
\text { (Fukuda } \text { et al., 1997) } \\
\text { (Paraskeva et al., 1999) } \\
\text { (Trotta et al., 2003) }\end{array}$ \\
\hline Exportin 2 (CAS) & Importin $\alpha$ & (Kutay et al., 1997) \\
\hline Exportin 3 (Exp-t) & tRNA & (Kutay et al., 1998) \\
\hline Exportin 5 (Xpo5) & $\begin{array}{l}\text { aa-tRNA } \bullet \text { eIF1A } \\
\text { dsRNA binding proteins } \\
\text { pre-miRNA }\end{array}$ & $\begin{array}{l}\text { (Bohnsack et al., 2002) } \\
\text { (Brownawell and Macara, 2002) } \\
\text { (Bohnsack et al., 2004) }\end{array}$ \\
\hline Exportin 6 (Xpo6) & actin•profilin & (Stuven et al., 2003) \\
\hline Exportin 7 (Xpo7) & p50RhoGAP, $14-3-3 \sigma$ & (Mingot et al., 2004) \\
\hline \multicolumn{3}{|l|}{ Import receptors } \\
\hline $\operatorname{Importin} \beta(\operatorname{Imp} \beta)$ & $\begin{array}{l}\text { Ribosomal proteins } \\
\text { HIV Rev, HIV Tat proteins } \\
\text { Snurportin•m }{ }_{3} \text { Gcap-UsnRNPs } \\
\text { XRIP } \alpha \cdot R P A\end{array}$ & $\begin{array}{l}\text { (Jakel and Gorlich, 1998) } \\
\text { (Truant and Cullen, 1999) } \\
\text { (Huber et al., 1998) } \\
\text { (Jullien et al., 1999) }\end{array}$ \\
\hline Importin $\beta$ / importin $\alpha$ & Classical NLS cargos & $\begin{array}{l}\text { (Gorlich et al., 1994) } \\
\text { (Gorlich et al., 1995) }\end{array}$ \\
\hline Importin $\beta$ / importin 7 & Histone $\mathrm{H} 1$ & (Jakel et al., 1999) \\
\hline Transportin (Impß-2) & $\begin{array}{l}\text { hnRNP M9 } \\
\text { Ribosomal proteins } \\
\text { Histones } \\
\text { SRP19 }\end{array}$ & $\begin{array}{l}\text { (Pollard et al., 1996) } \\
\text { (Jakel and Gorlich, 1998) } \\
\text { (Muhlhausser et al., 2001) } \\
\text { (Dean et al., 2001) }\end{array}$ \\
\hline Transportin-SR & SR proteins & (Kataoka et al., 1999) \\
\hline Importin 4 (Imp4) & Ribosomal proteins & (Jakel et al., 2002) \\
\hline Importin 5 (Imp5) & $\begin{array}{l}\text { Ribosomal proteins } \\
\text { histones }\end{array}$ & $\begin{array}{l}\text { (Jakel and Gorlich, 1998) } \\
\text { (Muhlhausser et al., 2001) }\end{array}$ \\
\hline Importin 7 (Imp7) & $\begin{array}{l}\text { Ribosomal proteins } \\
\text { histones }\end{array}$ & $\begin{array}{l}\text { (Jakel and Gorlich, 1998) } \\
\text { (Muhlhausser et al., 2001) }\end{array}$ \\
\hline Importin 8 (Imp8) & $\begin{array}{l}\text { SRP19 } \\
\text { Argonaute proteins }\end{array}$ & $\begin{array}{l}\text { (Dean et al., 2001) } \\
\text { (Weinmann et al., 2009) }\end{array}$ \\
\hline Importin 9 (Imp9) & $\begin{array}{l}\text { Histones } \\
\text { Ribosomal proteins }\end{array}$ & $\begin{array}{l}\text { (Muhlhausser et al., 2001) } \\
\text { (Jakel et al., 2002) }\end{array}$ \\
\hline Importin 11 (Imp11) & $\begin{array}{l}\text { UbcM9 } \\
\text { Ribosomal protein L12 }\end{array}$ & $\begin{array}{l}\text { (Plafker and Macara, 2000) } \\
\text { (Plafker and Macara, 2002) }\end{array}$ \\
\hline \multicolumn{3}{|l|}{ Bidirectional receptors } \\
\hline Importin 13 (Imp13) & $\begin{array}{l}\text { Mago-Y14, hUBC9 (import) } \\
\text { eIF1A (export) }\end{array}$ & (Mingot et al., 2001) \\
\hline Exportin 4 (Xpo4) & $\begin{array}{l}\text { eIF5A, SMAD3 (export) } \\
\text { Sox2, SRY (import) }\end{array}$ & $\begin{array}{l}\text { (Lipowsky et al., 2000) (Kurisaki } \\
\text { et al., 2006) } \\
\text { (Gontan et al., 2009) }\end{array}$ \\
\hline
\end{tabular}




\subsubsection{Structural features of $\operatorname{Imp} \beta$ family NTRs}

All members of the Imp $\beta$ family of NTRs are sequence related to importin $\beta$. Additionally, they all share common features like their large sizes (90-140 kDa), relatively acidic isoelectric points (4.0-6.0), their ability to bind Ran (Gorlich et al., 1997), their ability to interact with FG nups (Iovine et al., 1995; Bayliss et al., 2000; Bednenko et al., 2003) and with phenyl sepharose matrix (Ribbeck and Gorlich, 2002). NTRs also share a common overall architecture. The smallest structural unit is the HEAT repeat, named after the first class of proteins identified to contain them: huntingtin, elongation factor 3 , the PR65/A subunit of protein phosphatase 2A (PP2A) and the lipid kinase TOR (Andrade and Bork, 1995). A HEAT repeat is composed of two antiparallel alpha helices connected by a short intrarepeat linker. NTRs are usually made up of $\sim 20$ tandem HEAT repeats, all arranged in a slightly shifted angle to give rise to a right-handed superhelix (Figure 2-4). If the helices of a HEAT repeat are denoted as A and B; in an NTR, A helices face outward and B helices face inward. The helices contain hydrophobic residues that form the intra- and inter-repeat interfaces, which also might represent the hydrophobic pockets that are necessary for the interaction with FG nups. Despite their similarities, NTRs share surprisingly low overall sequence homology (sequence identity is around 8-15\%). The highest sequence homology is observed in the N-terminus, which the most acidic region and accounts for the Ran binding activity (Gorlich et al., 1997).

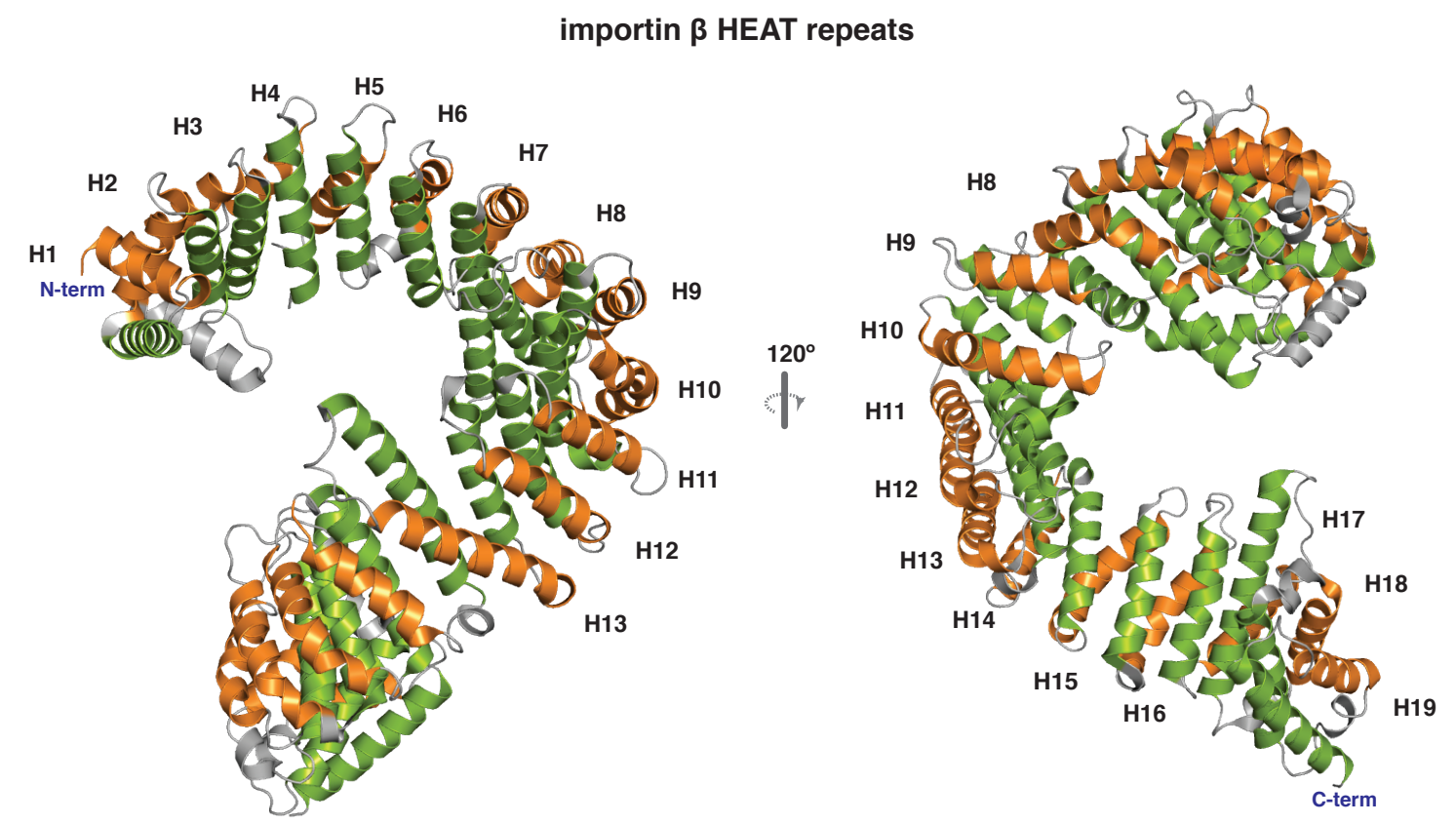

Figure 2-4 HEAT repeat architecture of Importin beta

Importin beta from the structure 2BKU (importin beta - RanGTP) (Lee et al., 2003) is depicted in two different orientations to visualize the HEAT repeat structure and the overall solenoid form. The HEAT repeats are numbered 
starting from the N-terminus. The A helices of each repeat (shown in orange) faces the outside of the solenoid, whereas the B helices (shown in green) line up the inner surface. The intra- and inter-repeat loops are colored in gray. $\operatorname{Imp} \beta$ has 19 HEAT repeats, but this number can vary in other NTRs (CRM1 has 21 HEAT repeats).

\subsubsection{Nuclear transport signals}

As mentioned earlier, the facilitated transport is a tightly controlled process. Where some NTRs carry only one specific cargo, like CAS (importin $\alpha$ ) (Kutay et al., 1997) and Exportin 6 (actin) (Stuven et al., 2003); some other NTRs recognize a large variety of cargos, such as CRM1 (Monecke et al., 2009) and Importin $\alpha / \beta$ (Gorlich et al., 1995). NTRs that transport many different proteins utilize certain signals to recognize their cargo, termed as nuclear import and nuclear export signals.

The first nuclear localization signal (NLS) to be identified was from the Large T-antigen of Simian Virus 40 (Kalderon et al., 1984a, 1984b). This sequence was a short patch containing basic amino acids: PKKKRKVE. Another type of basic NLS was later identified in Xenopus laevis nucleoplasmin (Robbins et al., 1991). In this case, the NLS consisted of two basic patches separated by a short spacer. The "monopartite" SV40 type and the "bipartite" nucleoplasmin type signals are collectively referred as the classical NLSs (cNLS). These sequences, when fused to cytoplasmic proteins, were enough to result in a nuclear localization. Later, many other nuclear proteins have been identified with similar basic patches. The mechanism of the Imp $\alpha / \beta$ mediated import was understood much later than the identification of the signals that drive proteins into the nucleus. The cNLSs are recognized by importin $\alpha$, which in turn interacts with importin $\beta$ through its importin $\beta$ binding (IBB) domain (Gorlich et al., 1995, 1996a). Considering that most DNA binding motifs consist of exposed basic patches, it is an elegant mechanism to use basic patches for nuclear import in order to sort such DNA-interacting proteins to nucleus via their functional domains. Another function that importins (Imp4, Imp5, Imp7, Imp9, $\operatorname{Imp} \beta$ ) fulfill by recognizing exposed basic patches in ribosomal proteins and histones is to prevent them from ionic aggregation in the cytoplasm (Jakel et al., 2002). In that way, importins function as chaperones for basic patches, like heat shock proteins for hydrophobic regions.

CRM1 is the main export receptor in the cell, responsible for the nuclear exclusion of many proteins and complexes that vary in size and function. CRM1 cargos, as versatile as they are, share a common Leu-rich signal that confer binding to CRM1, the classical 
nuclear export signal (NES). The first NES was discovered in the Protein Kinase A Inhibitor (PKI): a short stretch of interspersed hydrophobic amino acids was found to be responsible for nuclear exclusion (Wen et al., 1994). Another type NES was identified in the HIV protein Rev, which had a different spacing of the hydrophobic residues (Fischer et al., 1995; Wen et al., 1995). Later on, other proteins with similar hydrophobic stretches have been shown to bind CRM1. CRM1 has a hydrophobic cleft created by the HEAT repeats 11 and 12 and the NES nicely fits into this hydrophobic groove (Monecke et al., 2009). A more comprehensive study later revealed the structural basis for the NES-CRM1 interaction (Guttler et al., 2010). This study revised the known consensus sequences so far by showing that there is another hydrophobic residue at the $\mathrm{N}$ terminus of the known stretch involved in the binding. It also showed that the unstructured PKI and Rev type NESs assume different conformations to fit their hydrophobic residues to the hydrophobic pockets of the CRM1 cleft.

The classical nuclear transport signals do not explain all nuclear transport events. The NTRs with a limited number of cargos usually make extensive surface contacts with their cargo. Hence, one can talk about a three dimensional recognition of the substrate. These interactions have to be unraveled individually to understand the dynamics of the cargo and the respective NTR.

\subsubsection{Cargo recognition by NTRs}

The helical construction of NTRs makes them inherently flexible, which is core to their cargo recognition (Conti et al., 2006; Cansizoglu and Chook, 2007). Exportins accommodate RanGTP and their cargo at the same time, where the binding of one increases the NTR affinity towards the other. The cooperativity in cargo and Ran binding is caused by direct interactions of Ran and the cargo, and by the conformational changes throughout the molecule. CRM1 presents a special example where RanGTP and the cargo do not contact each other in the ternary complex (Monecke et al., 2009). Ran binds to the inner surface of the NTR, whereas cargo is accommodated on a cleft on the outer surface. Despite the positional distance of the cargo and RanGTP, CRM1 can only bind cargo when Ran is bound to it. The conformational changes upon Ran binding are propagated via flexible HEAT repeats, such that the hydrophobic cleft at the other end of the molecule assumes an open conformation for cargo binding. 
For other NTRs with specific cargos such as CAS or Exportin-t, cargo (imp $\alpha$ and tRNA respectively) is accommodated on the inner surface of the molecule, where it also has direct contacts with Ran (Matsuura and Stewart, 2004; Cook et al., 2009). This direct interaction contributes greatly to the cooperativity of cargo and Ran binding to the NTR (Kutay et al., 1997, 1998). In addition to the direct contact of Ran, the NTRs themselves undergo drastic conformational changes to accommodate the cargo (Guttler and Gorlich, 2011). Exportin 6 is also a very specific NTR, whose only known cargo is actin•profilin complex. Whether Exportin 6 engulfs its cargo like CAS and Exportin-t, or whether actin contacts Ran in the export complex remains to be elucidated.

In most cases, the way an NTR interacts with its cargo can not be pre-determined without structural evidence. The flexible construction of NTRs enables them to assume very different conformations to specifically interact with a given cargo. Importin $\beta$, for example, has been crystallized with many different cargos so far, and found to assume a different conformation each time (Vetter et al., 1999; Lee et al., 2003; Mitrousis et al., 2008; Choi et al., 2014). This holds true for other NTRs as well. The crystallographic data shows that cargo recognition by NTRs can be quite different than another (Figure 2-5). This makes it impossible for us to predict any mechanisms for NTR-cargo interaction without structural evidence. To date, several NTRs have been structurally characterized, however, there are still many of them that are waiting to be investigated. How exportin 6 recognizes actin will be the main question we will try to address in this study. 


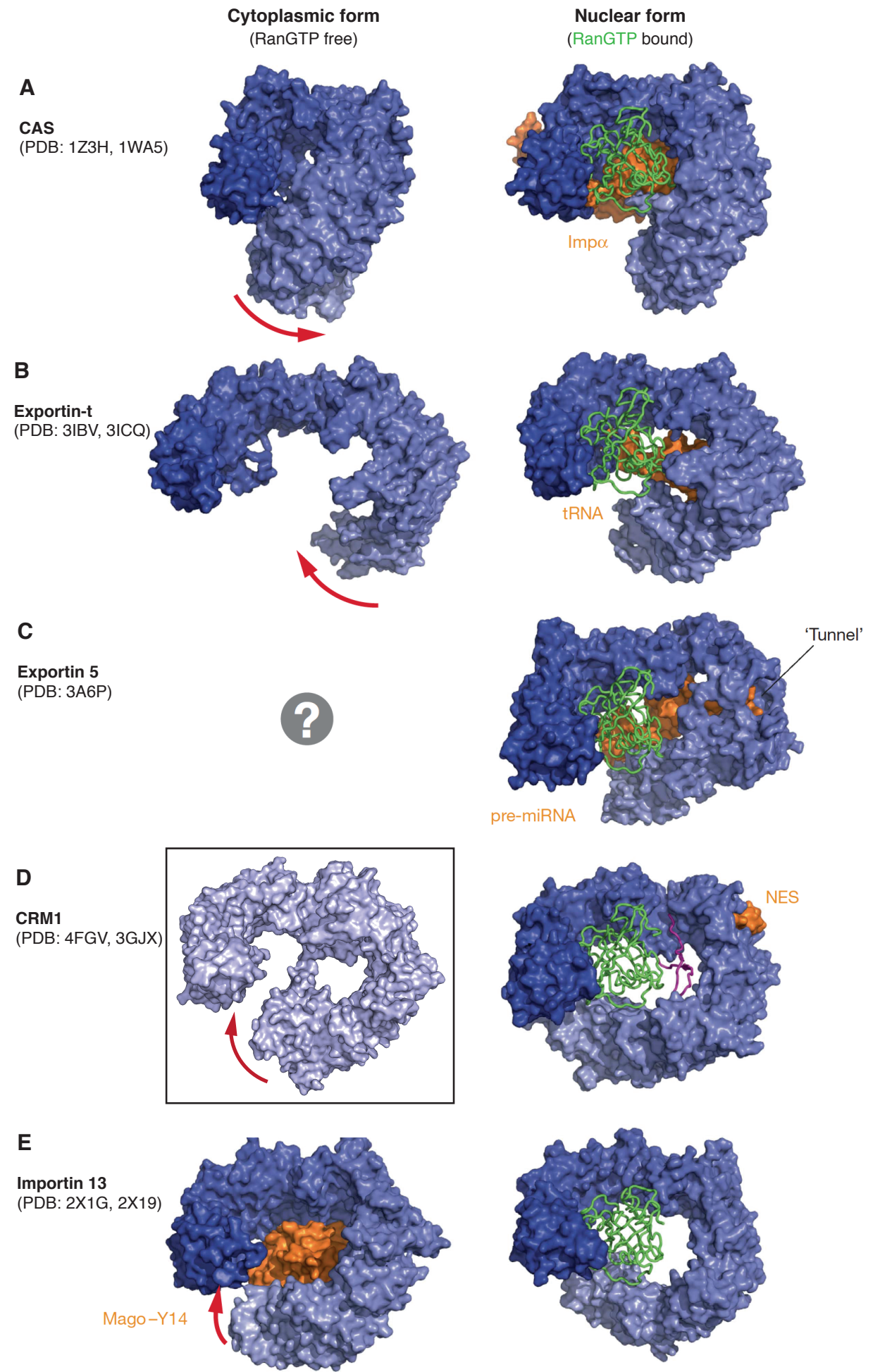

Figure 2-5 Comparison of structurally characterized exportins in cytoplasmic and nuclear states

This figure (Guttler and Gorlich, 2011) shows a structural comparison of the exportins that are structurally characterized. The receptors are shown in blue surface representation, Ran is shown in green and the cargo is shown in orange. The structure of the cargo-free CRM1 (Monecke et al., 2013) is added to the figure for complementation. The direction of movement upon cargo binding is indicated with red arrows on the left panel. Despite their similar constructions, exportins here are shown to assume drastically different conformations during the recognition of the cargo. In its free form CAS has a closed conformation, whereas Exportin-t is widely open. CAS opens up to accommodate Ran and Impa, whereas Exportin-t assumes a more packed conformation when it is bound to Ran and tRNA. Also note that the extent of surface contacts of the cargo with exportin and Ran is different for each complex. 


\subsection{Actin}

Actin is one of the major constituents of the cytoskeleton and also one of the most abundant proteins found in all eukaryotic cells. The main function of actin is to polymerize into microfilaments that form the cytoskeleton, and it is essential for cellular functions like cell division, vesicle trafficking, migration and cell shape regulation. Actin is involved in more protein-protein interactions than any other known protein, and has very complex dynamics as the main cytoskeletal component. It would therefore be a tremendous task to introduce actin from every aspect. For this reason, I will mainly focus on the isoforms, structure, binding partners and the nucleocytoplasmic distribution of actin in the following sections and refer to reviews for further details.

\subsubsection{Isoforms of actin}

Actin is invariably present in all eukaryotic cells with a striking degree of conservation. It is also suggested to be one of the ancestral genes of the last common ancestor prokaryotes and eukaryotes shared (Erickson, 2007). Prokaryotic homologs of actin (MreB, FtsA, ParM) do have little sequence homology to eukaryotic actin, but they share a strikingly similar fold (Shaevitz and Gitai, 2010). Mammals and birds have six isoforms that arose by gene duplications, which share more than 93\% sequence identity (Perrin and Ervasti, 2010). The isoforms are expressed in a regulated and tissue specific manner and despite their extreme conservation, show divergent properties that contribute to the varying functions of actin in different tissues (Perrin and Ervasti, 2010). All $\alpha$ isoforms $\left(\alpha_{\text {skeletal }}\right.$, $\left.\alpha_{\text {cardiac }}, \alpha_{\text {smooth }}\right)$ and $\gamma_{\text {cardiac }}$ actin are mainly expressed in the respective muscle tissues, whereas $\beta$ and $\gamma$ actin are expressed ubiquitously in muscle and nonmuscle cells. The isoforms $(\alpha, \beta, \gamma)$ differ only by subtle amino acid changes, most of them confined to the extreme N-terminus of the protein (Herman, 1993). The isoactins may differ in their binding affinities to certain actin binding proteins (ABPs) such as cofilin, thymosin $\beta 4$ and profilin, show differential localization patterns within the same cell and differ in their polymerization rates under non-physiological conditions (reviewed in (Herman, 1993; Perrin and Ervasti, 2010)

\subsubsection{Structure of the actin monomer}

Actin is a single polypeptide chain of 375 amino acids that makes up a $42 \mathrm{kDa}$ globular protein and shares a unique fold with other members of its ATPase superfamily: sugar kinases and Hsp70 (Bork et al., 1992). The protein has two major domains that are 
connected to each other by a flexible hinge region. The domains are termed as small and large or outer and inner domains (according to their positions in an actin filament) respectively. These two domains are further divided into subdomains, which are historically referred as subdomains 1-4 (Figure 2-6). The hinge region between the domains creates two clefts that are essential to actin function: the nucleotide cleft and target-binding (hydrophobic) cleft (Dominguez and Holmes, 2011). The nucleotide cleft accommodates the adenine nucleotide (ATP or ADP) and the divalent cation $\left(\mathrm{Mg}^{++}\right.$in vivo), which in turn provides a further contact between the subdomains 2 and 4 (Dominguez and Holmes, 2011). The smaller cleft between the subdomains 1 and 3 is lined with hydrophobic residues, which serve as a docking side for most of the actin binding proteins (ABPs) (Dominguez, 2004). The two clefts communicate with each other via conformational changes of the subdomains relative to each other, which is essential for the modulation of the nucleotide and ABP binding affinities. DNaseI is an exceptional ABP, which binds to a specific loop in the subdomain 2, D-loop (39-51), rather than the hydrophobic cleft (Dominguez and Holmes, 2011).

\subsubsection{The actin filament and regulation of polymerization}

Actin monomers can self assemble in a directional manner to form long filaments. An actin filament can be considered as two arrays of actin molecules (protofilaments) that wind around each other to form a right-handed helix with a long pitch (Alberts et al., 2007). Similar to the actin monomer (G-actin, globular actin) the resulting actin filaments (Factin) are polar in nature (Figure 2-6). The two ends of an actin filament polymerize at different rates: the fast growing end is termed as the plus (barbed) end and the slow growing end is called minus (pointed) end (Pollard, 1986). Polymerization, like crystallization, starts with energetically unfavorable nucleation step. Once a stable core is formed, filaments grow rapidly until equilibrium is reached between monomers and filaments. The free monomer concentration at this equilibrium is referred as the critical concentration $\left(\mathrm{C}_{\mathrm{c}}\right)$. All actin monomers above this concentration polymerize into filaments (Carlier, 1990). 
A) The actin monomer

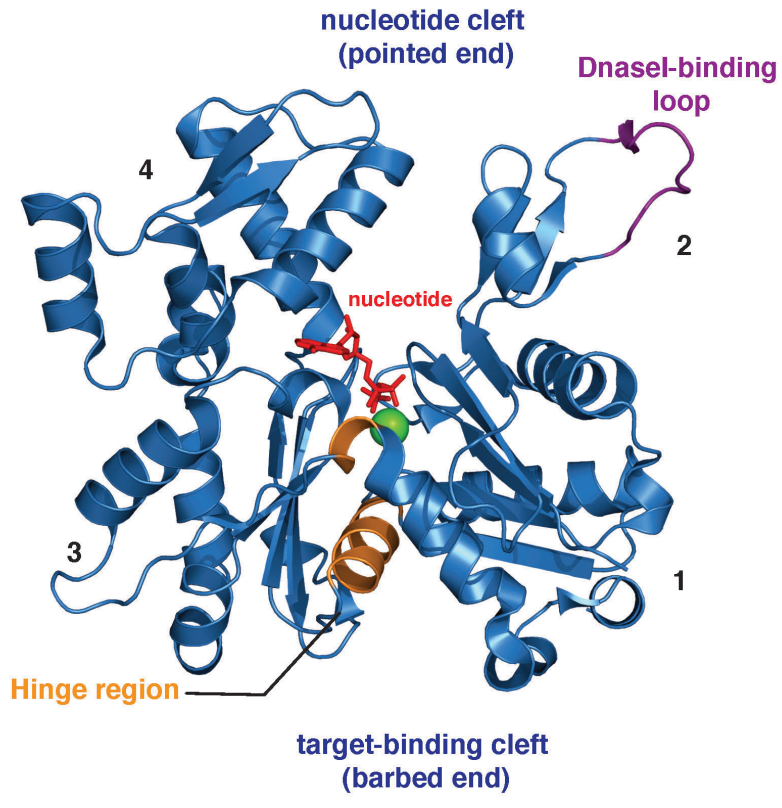

B) actin-profilin complex

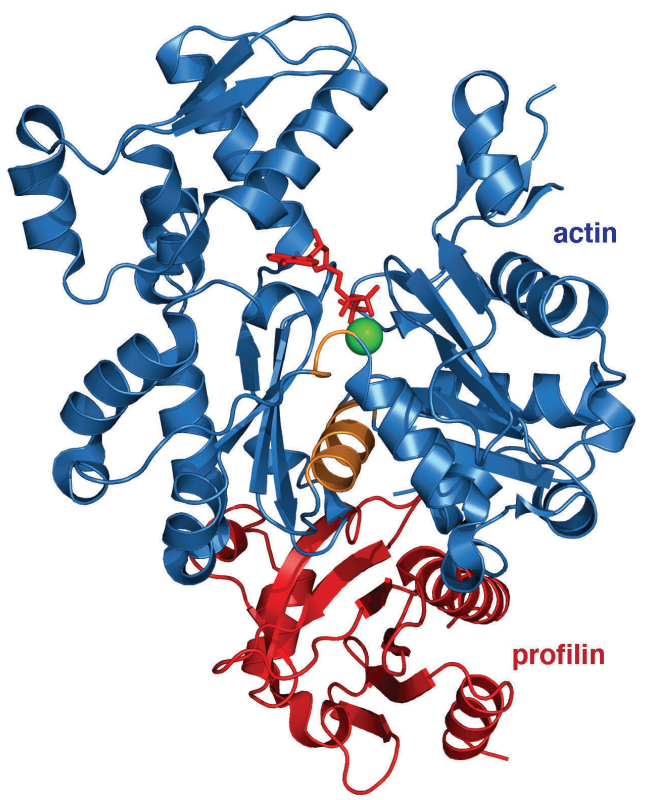

C) The actin filament

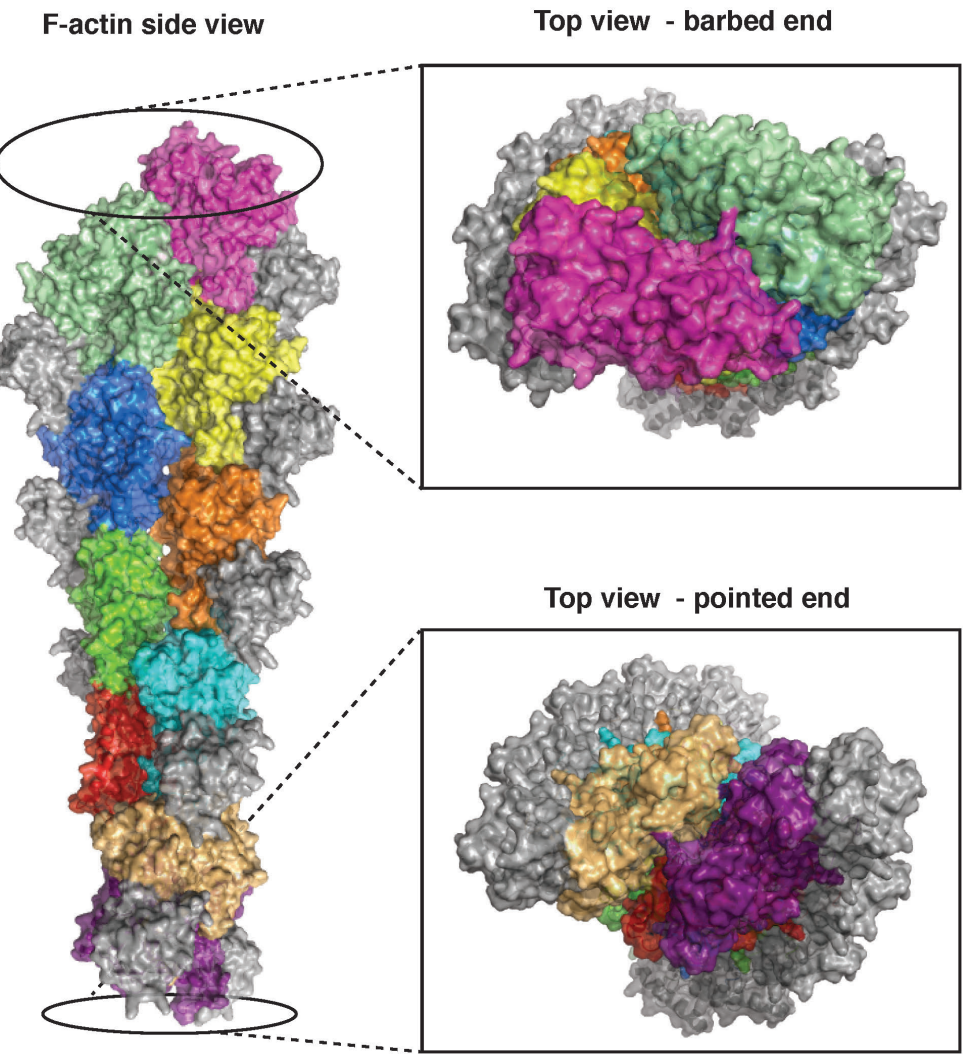

Figure 2-6 Structure of G-actin, profilactin and the actin filament

A) Structure of the skeletal alpha actin monomer (G-actin) PDB-ID: 2A42 (actin-DNaseI complex)(Chereau et al., 2005). The bound nucleotide (ATP) and the cation $\left(\mathrm{Ca}^{++}\right.$here $)$are shown in red and green respectively. Hinge region, where the subdomains 1 and 3 connect, is shown in orange. DNase-I binding loop (D-loop) is shown in violet. The cleft between subdomains 2 and 4 is the nucleotide cleft, and this also constitutes the pointed (-) end of the actin monomer. The cleft opposite to the nucleotide cleft is the hydrophobic target binding cleft, where most ABPs bind actin. B) Structure of alpha actin profilin complex (also referred as profilactin) PDB-ID: 2PBD (Ferron et al., 2007). Profilin binds to the target binding cleft at the barbed end of an actin molecule. Note that the D-loop is disordered in this structure since it is not stabilized by DNaseI. C) Structure of the actin filament (F-actin) from the model with PDB-ID 3LUE(Galkin et al., 2010). In this model, the F-actin filament is decorated with actin binding (CH1) domain of $\alpha$-actinin, which binds to the 
subdomains 1-2 near the D-loop. The CH1 domains, shown in gray, indicates the orientation and the twist of the actin monomers (each in different colors) within the filament. On the right, both ends of a filament are shown in close-up top views.

Polymerization triggers conformational changes on the actin monomer, which increase its intrinsic ATPase activity. Nucleotide is rapidly hydrolyzed and inorganic phosphate is released at a much slower rate. The nucleotide state marks the age of an actin filament as the release of phosphate causes destabilization of the actin-actin interactions and structural changes in the filament (Carlier, 1990). Treadmilling, the assembly of new (ATP) subunits at the barbed end and simultaneous dissociation of old (ADP) subunits at the pointed end, occurs at sufficiently high monomer concentrations, as a result of the differences in the association kinetics at the two ends of a filament and irreversible ATP hydrolysis (Wegner, 1982). Keeping monomeric actin concentration high enables the cell to rearrange the cytoskeleton in a very short time upon external stimuli.

The actin $\mathrm{C}_{\mathrm{c}}$ for polymerization depends on the bound nucleotide $\left(\mathrm{C}_{\mathrm{c}} \mathrm{ATP}<\mathrm{C}_{\mathrm{c}} \mathrm{ADP}+\mathrm{P}_{\mathrm{i}}<\right.$ $\left.\mathrm{C}_{\mathrm{c}} \mathrm{ADP}\right)$, the nucleotide bound divalent cation $\left(\mathrm{C}_{\mathrm{c}} \mathrm{Mg}^{++}<\mathrm{C}_{\mathrm{c}} \mathrm{Ca}^{++}\right)$and the type and concentration of the ions in the solution (Kang et al., 2013). Salts and temperature lower the actin $\mathrm{C}_{\mathrm{c}}$, such that addition of potassium, magnesium (or calcium) and ATP will already initiate polymerization of an actin solution (Spudich and Watt, 1971; Wegner, 1982). In vitro, actin $\mathrm{C}_{\mathrm{c}}$ near physiological conditions are as low as $0.1-0.2 \mu \mathrm{M}$, whereas in vivo cellular monomeric actin concentration can reach 50-200 $\mu \mathrm{M}$ (Alberts et al., 2002). The cell invests considerable amount of resources to regulate the actin cytoskeleton dynamics. There are a myriad of actin binding proteins that are involved in functions such as keeping actin monomeric (profilin, cofilin, thymosin $\beta 4$ ), filament nucleation and branching (formins, Arp2/3 complex), filament elongation at the barbed end (formins), capping of the filament ends (capping protein, CapZ, tropomodulin), filament severing/depolymerization (gelsolin, severin, cofilin), filament stabilization (tropomyosin), filament bundling/crosslinking ( $\alpha$-actinin, spectrin, dystrophin, utrophin) and motor proteins that move on actin filaments (myosins) (Pollard and Cooper, 1986; dos Remedios et al., 2003; Pollard and Borisy, 2003; Pollard and Cooper, 2009).

The actin cytoskeleton is not only regulated by actin binding proteins, but also by a number of small molecules. Plants, fungi, bacteria and some marine organisms produce toxins that can act on the actin cytoskeleton. Some of these toxins bind to actin monomers and cause rapid actin depolymerization (Latrunculins, Cytochalasins, Swinholide A, etc), 
some others bind to actin filaments and stabilize them (Phalloidin, Jasplakinolide, etc) (Allingham et al., 2006). These toxins are intensively used in actin research: cytochalasins and latrunculins as actin depolymerization drugs (Cooper, 1987; Morton et al., 2000), fluorescently labeled phalloidin for actin filament visualization (Wulf et al., 1979).

\subsubsection{Profilin}

Among all actin-binding proteins, profilin will be of special importance for this project. It is not only an important regulator of actin polymerization, but also is the cofactor for actin nuclear export. Profilin is a small $(15 \mathrm{kDa})$ protein, which was first identified in a 1:1 complex with actin as an inhibitor for DNaseI (Carlsson et al., 1976, 1977). Figure 2-6 shows the crystal structure of profilactin complex. In mammals there are four isoforms of profilin, encoded by different genes either ubiquitously (profilin I) or in a tissue specific manner (profilin II - brain, profilin III,IV - testis) (Birbach, 2008). The first function attributed to profilin was inhibition of polymerization (Carlsson et al., 1977). Indeed, profilin is present in eukaryotic cells from protozoa through humans in varying but high concentrations (20-100uM) (dos Remedios et al., 2003; Dominguez and Holmes, 2011) and is responsible for a large portion of the monomeric actin pool. But a true sequestering factor for actin is thymosin $\beta 4$ (Safer et al., 1991). Functions of profilin are much more diverse and complex. Profilin inhibits actin nucleation (Reichstein and Korn, 1979), catalyzes nucleotide exchange (Mockrin and Korn, 1980) and inhibits ATP hydrolysis on actin (Tobacman and Korn, 1982), promotes ATP-actin addition to the barbed end of actin filaments (Tilney et al., 1983). Profilin binds to Poly-L-proline sequences (Tanaka and Shibata, 1985), through which it is associated to formins like VASP (Ferron et al., 2007). Its high affinity to $\mathrm{PIP}_{2}$ makes profilin a likely mediator between the receptor tyrosine kinase signaling pathways and the cytoskeleton (Lassing and Lindberg, 1985). Last but not least, profilin is an essential binding partner for the nuclear export of actin (Stuven et al., 2003).

\subsubsection{Nucleocytoplasmic distribution of actin}

Actin is a protein with prominent cytoplasmic functions. Together with microtubules, actin filaments (microfilaments) make up the cytoskeleton. In non-muscle cells they are organized in bundles and networks. The actin cytoskeleton shapes the cell, is essential for cytokinesis, provides cellular motility (lamellipodia, filopodia, pseudopodia) and forms the tracks for intracellular transport of macromolecules, vesicles, even organelles. The cytoskeleton is very dynamic and responds to a variety of intra- and extracellular stimuli. 
In muscle cells, actin and actin motor protein myosin form the contractile bundles (myofibrils) together with several other ABPs, to provide force and motion. Here, the actin filaments are not nearly as dynamic as the cytoskeleton.

Actin with its globular fold and $42 \mathrm{kDa}$ molecular weight is at the limit of the passive diffusion barrier of the NPC (Mohr et al., 2009). Indeed, actin was shown to diffuse passively into the nucleus (De Robertis et al., 1978). Another route for actin into the nucleus is the breakdown of the nuclear envelope during mitosis, which causes an intermixing of the nuclear and cytoplasmic contents. More recently, an active nuclear import pathway for actin by Imp9 has been suggested (Dopie et al., 2012), but there is still no convincing biochemical data for this interaction. Despite the ability of actin to enter the nucleus, the predominant cytoplasmic localization of actin in vast majority of the cells indicates an active export mechanism. Actin was first claimed to be exported by CRM1 via two potential NESs (Wada et al., 1998). But in reality these suggested NESs correspond to rigid folds in the three dimensional structure of the protein, which would be inaccessible for CRM1 binding. Indeed, a couple of years later, a new NTR, exportin 6, was identified and shown to be the only responsible factor for actin nuclear export (Stuven et al., 2003).

While the cytoplasmic functions of actin have been understood quite well, the presence and the role of actin in the nucleus have been debated in the field since a long time. First reports of nuclear actin in somatic cells (Ohnishi et al., 1964), and amphibian oocytes (Clark and Merriam, 1977) date back almost 50 years by now. While the nuclear actin network of amphibian oocytes is widely supported by further research; the presence, form and functions of actin in the somatic cell nuclei is still under heavy debate. There are various reports for actin being involved in nuclear functions such as transcriptional regulation by binding to RNA polymerases (Fomproix and Percipalle, 2004; Hofmann et al., 2004; Hu et al., 2004; Philimonenko et al., 2004), chromatin remodeling (Zhao et al., 1998) and histone modification (Sjolinder et al., 2005). However, these studies fail to elucidate the molecular mechanisms of actin function in the mentioned complexes. A more convincing study shows that actin regulates the nuclear transport and the activity of the transcriptional activator MAL (Vartiainen et al., 2007).

Even if actin is involved in nuclear functions, the form of actin (monomeric or filamentous) in the nucleus remains unsettled so far. To date, no phalloidin stainable filaments have been observed in the somatic nuclei (Hofmann and de Lanerolle, 2006), and 
other observations with GFP-actin indicate an immobile pool of actin (McDonald et al., 2006), which does not necessarily refer to filamentous actin as we know it. Even in the presence of the active export mechanism, not all actin molecules can be removed from the nucleus. The facilitated transport can function within the limits of the RanGTP gradient (1000 fold, (Gorlich et al., 2003)), hence the actin concentration in the nucleus is probably kept at $0.1-1 \%$ of cytoplasmic G-actin levels. Also, any actin molecule that is bound to other nuclear binding partners is out of the reach of Exportin 6. This residual amount of actin in the nucleus is sufficient to explain the regulatory functions that G-actin might be involved. However, it efficiently prevents the formation of the filaments similar to the cytoplasmic ones.

\subsubsection{Exportin 6-mediated actin nuclear export pathway}

Exportin 6 (Xpo6) is conserved in all vertebrates and insects and even in the evolutionary distant slime mold Dictyostelium discoideum; however, no Xpo6 has been identified in fungi, plants and in C. elegans (Stuven et al., 2003). BLAST analyses show that CRM1 is the closest relative of Xpo6 within the Imp- $\beta$ superfamily of NTRs in terms of amino acid sequence. Xpo6 interacts with only a couple of functionally related proteins in a Ran dependent manner: $\beta$-actin, profilin, Mena, Vasp and mDia, which all are part of actin cytoskeleton (Stuven et al., 2003). From those, only actin and profilin are identified as the actual cargos. The identified export complex, Xpo6•Ran• $\beta$-actin•profilin, is reported to have a 1:1:1:1 stoichiometry. Mena, Vasp and mDia are all members of the formin family, which bear proline-rich profilin binding sites (Holt and Koffer, 2001) and might be recruited to the export complex via the Poly-Pro binding pocket of profilin. The sequence conservation between Xpo6 orthologs is rather low compared with other NTRs. Zebrafish (D. rerio) Xpo6 is $\sim 72 \%$ identical in amino acid sequence with the human protein, however the identity between Drosophila and human Xpo6 is as low as 20\% (Stuven et al., 2003). Despite the low conservation, the functionality across species is still conserved (Stuven et al., 2003). Xpo6 can export all human profilin isoforms (Stuven et al., 2003), $\beta$ $\gamma$ - $\alpha$ - isoforms of actin and even actin from yeast (Bohnsack et al., 2006).

The fact that Xpo6-mediated actin export is conserved in higher eukaryotes, indicates a selective pressure on keeping nuclear actin levels low in these organisms. There are however, some exceptions to the rule. Amphibian oocyes, as mentioned, accumulate large amounts of phalloidin stainable actin in their nuclei (Roeder and Gard, 1994). It was found that they maintain this nuclear actin pool by specifically blocking Xpo6 expression at 
translational level, which is only relieved during meiotic maturation (Bohnsack et al., 2006). The inhibition of actin nuclear export is essential for the stabilization of the giant nuclei $(400 \mu \mathrm{m}$ in diameter) of this cell type, as the microinjection of exogenous Xpo6 to the Xenopus oocyte nuclei caused immediate export of all actin and rendered the nuclei very fragile (Bohnsack et al., 2006). It is now known that there is an actin nucleoskeleton in Xenopus oocytes, whose function is not only the mechanical stabilization of the nuclei, but also proper segregation of the chromosomes and polar body extrusion during meiosis (Samwer et al., 2013).

When Xpo6 is silenced in Drosophila Schneider cells, intranuclear accumulation of rodshaped actin aggregates was observed (Stuven et al., 2003). Also, a lethal Drosophila mutant identified 25 years ago (Perrimon et al., 1989) turned out to have a defective Xpo6 gene. Recently, a genome-wide morphology RNAi screen has been performed in Drosophila S2R+ cells in parallel to a more focused RNAi screen in HeLa cells for all known actin regulatory proteins (Rohn et al., 2011). It has been shown that knockdown of Xpo6 and Drosophila profilin (chic) leads to the formation of phalloidin stainable actin rods within the nucleus. Several other studies showed that actin accumulation occurs as a result of the disruptions to the actin nuclear export pathway. In senescent cells, where RanGTP gradient is disrupted, nuclear actin accumulation is commonly seen (Park et al., 2011). ATP depletion (and concomitant GTP depletion) has caused rod-like actin accumulation in the nucleus of mouse cell lines (Domazetovska et al., 2007). DMSO treatment in amoeba, Dictyostelium and in HeLa cells (Fukui and Katsumaru, 1979; Osborn and Weber, 1980; Sanger et al., 1980), which probably breaks down the permeability barrier of the NPC (Ribbeck and Gorlich, 2002), results similarly in actin bundles in the nucleus. Accumulation of actin in these cases is only plausible if the main route of actin into the nucleus is passive diffusion. After the depletion of the RanGTP gradient, which is also an eventual result of the permeability barrier breakdown, all Randependent nucleocytoplasmic transport comes to a stall. With no active nuclear export mechanism to pump out, actin accumulates in the nucleus.

\subsubsection{Intranuclear rod myopathy}

A special case of nuclear actin accumulation is seen in patients suffering from intranuclear rod myopathies (IRM). IRM are muscle diseases that are associated with muscle weakness, hypotonia, hyporeflexia, a high-arched palate and autonomic dysfunctions, while the onset and the severity of the disease varies between patients; in many cases lethal in the early 
stages of life (Hutchinson et al., 2006; Kaimaktchiev et al., 2006; Chou et al., 2013; Nowak et al., 2013). IRM is characterized by the accumulation of rod-like actin structures in the nuclei of the muscle cells (Goebel and Warlo, 1997) (Figure 2-7). Each identified case of IRM so far revealed a mutation in the skeletal $\alpha$-actin gene (ACTA1) (Feng and Marston, 2009). To date there are roughly 180 known mutations of the human ACTAl gene (Laing et al., 2009). These mutations cause a wide range of muscle abnormalities, collectively referred as myopathies: nemaline myopathy (NM), actin myopathy (AM), core myopathy (CM), intranuclear rod myopathy (IRM), congenital fiber type disproportion (CFTD) (Feng and Marston, 2009; Laing et al., 2009). 13 mutations, causing amino acid changes in 12 different positions in ACTA1 have been associated with IRM so far (Goebel and Warlo, 1997; Laing et al., 2009). Where some of the mutations localize around the hinge region (Figure 2-7), it is hard to associate the mutations with the loss of a particular function of the molecule.
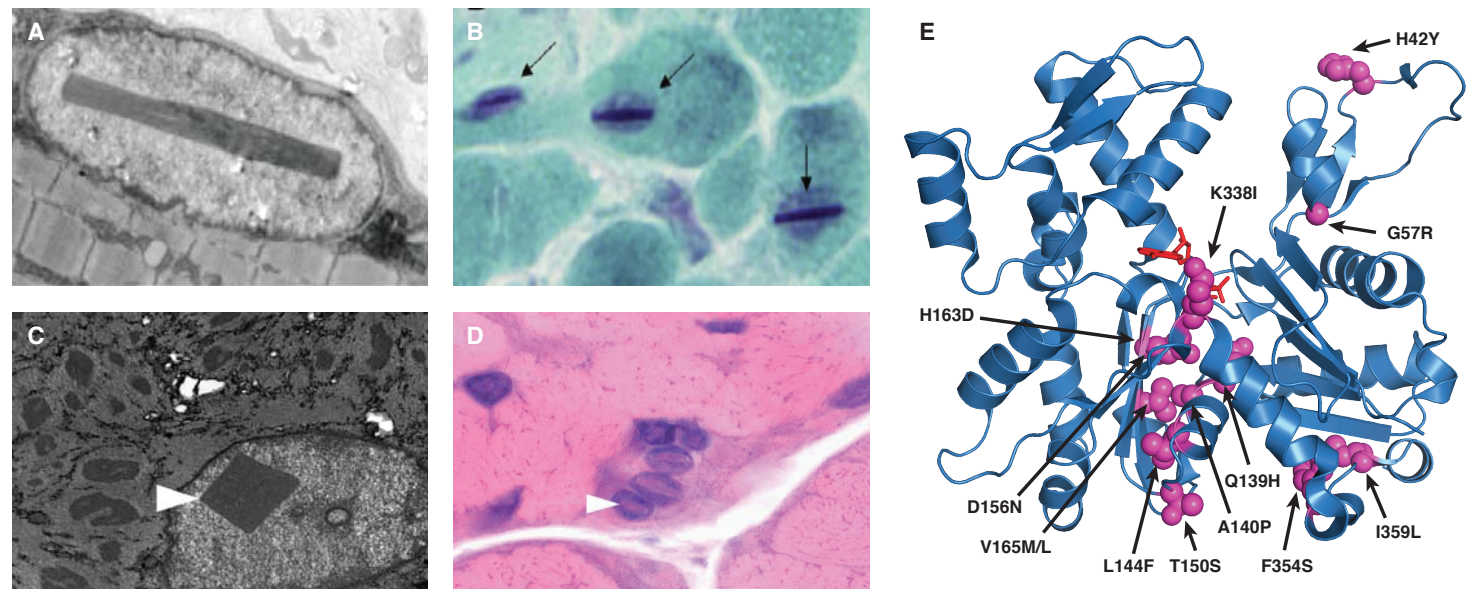

Figure 2-7 Intranuclear rod myopathy

A) Intranuclear rod caused by V165M mutation (Sparrow et al., 2003). B) Muscle biopsy from the left anterior tibial muscle, Gomori trichome staining, intranuclear rods are shown with arrows (Arai et al., 2009). C-D) muscle biopsy of a patient with V165M mutation c) electron micrograph; the intranuclear rod is indicated with a white arrowhead. d) hematoxylin-eosin staining; all nuclei contain actin rods. (Hutchinson et al., 2006) E) 13 known IRM mutations illustrated on the monomeric alpha actin (PDB-ID: 2A42). Note that most of the mutations localize near the hinge region.

Why these mutations lead to an exclusive nuclear accumulation in the first place still remains to be elucidated. There are several hypotheses on the mechanisms that lead to nuclear accumulation of IRM mutants, some of which are based on flawed arguments. Several studies hypothesized that some IRM mutations interfere with the so-called NESs of actin, impairing the nuclear export (Ilkovski et al., 2004; Kaimaktchiev et al., 2006; Ravenscroft et al., 2011), others hypothesized an increased trafficking into the nucleus (Domazetovska et al., 2007) or alterations in the polymerization and nucleotide binding 
properties due to the mutations in the hinge region (Sparrow et al., 2003; Ravenscroft et al., 2011). Based on the conserved nuclear actin export pathway, we hypothesize that at least some of these mutations might compromise Xpo6 binding of actin, leading to a nuclear accumulation and consequent rod formation.

There are many open questions that need to be answered concerning the nucleocytoplasmic transport of actin. How does Xpo6 interact with its cargo on molecular level? Does the cargo and Ran interact in the complex? What kind of conformational changes occur in Xpo6 between the cargo-bound and free forms? Is profilin making contacts with Xpo6, or how exactly does it contribute to the recognition of actin by Xpo6? Are any of the IRM mutations in the Xpo6-actin binding interface? How else can they interfere with the Xpo6actin interaction? Can we design any profilin or Xpo6 mutants that can recognize IRM actins? These questions can only be addressed with structural information of the actin export complex at atomic resolution. For this reason, we aspired to structurally characterize the actin nuclear export complex by X-ray crystallography. We want to understand the dynamics of cargo recognition by Xpo6, the details of actin nucleocytoplasmic shuttling, and eventually elucidate the molecular mechanisms leading to IRM. 


\section{Results}

Crystallization of a macromolecule requires sample preparations of highest purity and homogeneity. Once this is achieved, one has to identify the conditions for crystallization and diffraction at an optimum resolution. This may represent a very narrow window in a vast amount of possible conditions. In this project, we set out to crystallize a fourcomponent complex (Xpo6•RanGTP•actin •profilin) with a size of $200 \mathrm{kDa}$. This certainly is a challenge on its own. There were however, other unanticipated obstacles we had to overcome on the way towards successful crystallization. First challenge was the low affinity of skeletal alpha actin for the other components of the complex. Later, we found out that the export complex was extremely salt sensitive in vitro. Also, the recombinant expression and purification of individual proteins, especially Xpo6, had to be optimized for better yield and purity. But the expression/purification optimizations will not be discussed here for the sake of brevity. The latest purification conditions are described in materials and methods section. In this section, we will rather focus on the formation of the export complex and sub-complexes in vitro, the biochemical properties of the export complex and the crystallization trials for the Xpo6•RanGTP complex, the free Xpo6 and the actin nuclear export complex.

\subsection{The actin isoforms differ in their affinity for the complex}

\subsubsection{First attempts with a-actin from skeletal muscle}

In our first attempts to assemble the export complex, we have used purified, tag-free proteins. Actin was purified from rabbit skeletal muscle as described in section 6.2.3. We simply mixed them in near stoichiometric ratios and incubated them in the cold room for 1.5 hours. Actin, profilin and Ran were added in 1.2 fold molar excess over Xpo6, in order to be able to saturate Xpo6. Later, the solution was exchanged to a low ionic strength buffer (50 mM Tris pH 7.5, $50 \mathrm{mM} \mathrm{NaCl}, 0.5 \mathrm{mM} \mathrm{MgCl}_{2}, 5 \mathrm{mM}$ DTT), centrifuged and applied to Superdex 200 10/30 gel filtration column, equilibrated with the same buffer. Figure 3-1 shows the gel filtration profile and the SDS PAGE analysis of the fractions. It is seen that the proteins indeed form a complex, and run together. However, the second most prominent finding is that the profilin is only weakly associated to the complex. By comparing the band intensities for Ran and actin, one can realize that also the association of actin to the complex is sub-stoichiometric. Profilin might be dissociating from the 
complex during the gel filtration, or there might be a pool that doesn't associate with actin and the complex to start with.
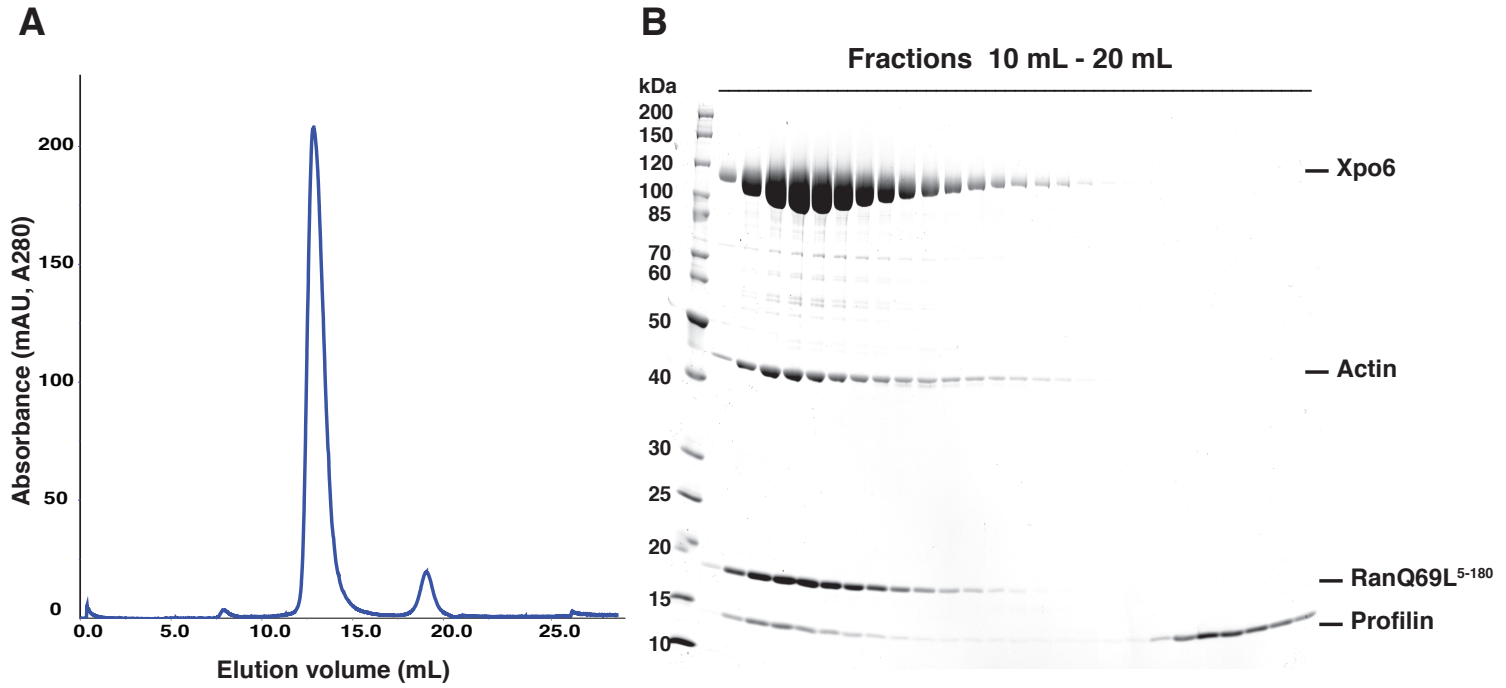

Figure 3-1 Export complex formation with alpha actin

A) The size exclusion chromatogram of the export complex on SD200 10/30 column equilibrated with $50 \mathrm{mM}$ Tris $\mathrm{pH}$ 7.5, $50 \mathrm{mM} \mathrm{NaCl}, 0.5 \mathrm{mM} \mathrm{MgCl} 2,5 \mathrm{mM}$ DTT. Y-axis shows the UV absorbance at $280 \mathrm{~nm}, \mathrm{X}$-axis shows the elution volume. B) Fractions of the gel filtration in (A) on a gradient SDS polyacrylamide gel. See text for experimental details.

Free actin above the critical monomer concentration can polymerize into filaments under suitable ionic conditions (Wegner, 1982; Carlier, 1990). Equal amounts of actin and profilin were present in the initial mixture and a fraction of profilin did not bind actin and eluted from the column as monomers. Since there was not an additional peak for actin in the chromatogram, the unbound actin most likely polymerized and was removed by centrifugation before gel filtration.

In our later experiments, we tried to improve the initial complex, by varying several parameters. We increased the actin and profilin concentrations in the complex formation, we compared actin purified rabbit and chicken skeletal muscle. We realized in our experiments that some components of the actin export complex have buffer requirements that are not compatible with each other. We observed a tendency of Ran to aggregate in low salt buffers, when it is not bound by NTRs. Actin on the other hand, tends to polymerize at salt concentrations of $50 \mathrm{mM}$ (Spudich and Watt, 1971), if it is not kept monomeric by profilin. Salt concentrations that are too low for unbound Ran are already too high for actin to be monomeric, which represents a major dilemma for the complex formation. We used low salt concentrations $(20 \mathrm{mM})$ and cytochalasin $\mathrm{B}(\mathrm{CytB}, 10 \mu \mathrm{M})$ in the reaction in order to prevent actin polymerization and improve complex formation. We could say that the source of $\alpha$ actin did not make any difference on the complex formation. 
Neither could we improve the complex stability by varying concentrations of the components or inhibition of actin polymerization.

\subsubsection{Non-muscle actins form a more stable complex with profilin}

The affinity problems with $\alpha$ actin led us to alternative sources for actin. In the literature, there are several studies showing that skeletal $(\alpha)$ actin has less affinity towards profilin than non-muscle actin isoforms $(\beta / \gamma)$ (Larsson and Lindberg, 1988; Ohshima et al., 1989; Vinson et al., 1998; Kinosian et al., 2000). We therefore decided to use HeLa cytoplasmic extract as a source for non-muscle actin. We first analyzed whether a stable profilactin complex could be formed with non-muscle actin. In a parallel experiment, we tested purified $\alpha$ actin (chicken) or cytoplasmic HeLa extract as an actin source. Profilin with an $\mathrm{N}$-terminal His-tag was used to pull actin from the solution. Actin was supplied in excess in both setups. Proteins were incubated in the presence of $5 \mathrm{mM}$ EDTA and $5 \mu \mathrm{M} \mathrm{CytB}$ for $1 \mathrm{~h}$ in cold room, and then bound to Ni-matrix for $2 \mathrm{~h}$ in a low salt buffer (10 mM Tris 7.5, $20 \mathrm{mM} \mathrm{NaCl}, 1 \mathrm{mM}$ DTT) in the presence of $5 \mathrm{mM}$ imidazole to reduce unspecific binding to the matrix. The unbound proteins were removed, and the bound profilin and profilinbound actin were eluted by the cleavage of the His-tag with SumoStar protease. Later the still Ni-bound fraction was post-eluted with imidazole. Figure 3-2 shows the result of the experiment. It is seen that with the same amount of profilin, more actin was pulled from the HeLa cytoplasmic extract compared to purified $\alpha$ actin.

Our experiment also represents a very elegant one-step method for purification of cytoplasmic actin and profilactin. Muscle actin can be purified in high-yield with a relatively straightforward method that relies on multiple rounds of polymerization and depolymerization (Spudich and Watt, 1971). However, the protocols for non-muscle actin purification have been very cumbersome and low in yield in comparison to muscle actin purification. Multiple purification steps such as $\mathrm{C}_{\gamma}$ alumina gel, DEAE chromatography, hydroxyapatite chromatography, ammonium sulfate precipitation and gel filtration had to be combined for the optimum purity (Lindberg, 1967; Carlsson et al., 1976; Gordon et al., 1976; Carlsson et al., 1977). Here we show that highly pure non-muscle actin and profilactin can be isolated from HeLa cytoplasmic extract in a one-step protocol. This method can also be used with tissue extracts other than HeLa cells. 


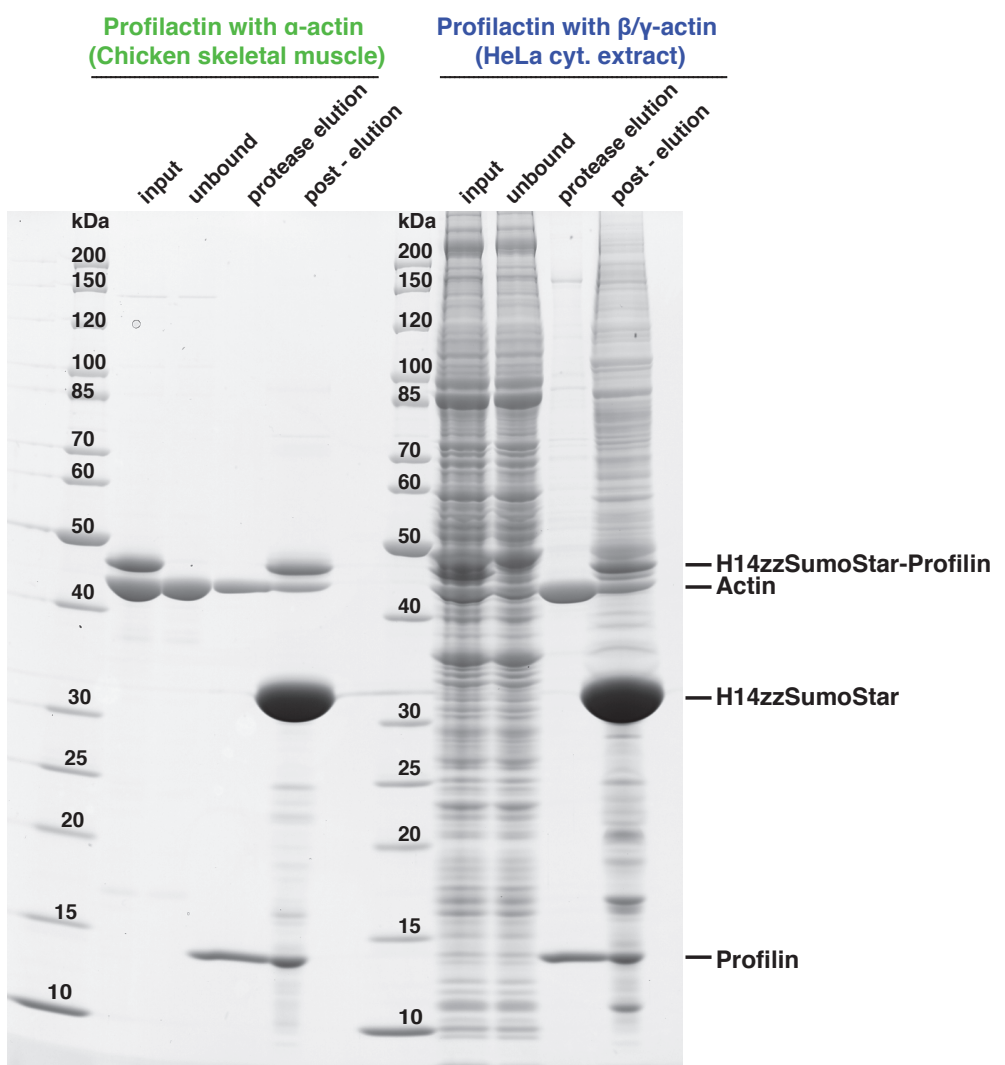

Figure 3-2 Profilactin with muscle and non-muscle actin isoforms

Formation of profilactin complexes with muscle and non-muscle actin isoforms. $5.2 \mathrm{nmol}$ His-tagged profilin was incubated with $13 \mathrm{nmol} \alpha$ actin or $500 \mu \mathrm{L}$ HeLa S10 extract. Binding was performed in $10 \mathrm{mM}$ Tris $7.5,20 \mathrm{mM} \mathrm{NaCl}$, $1 \mathrm{mM}$ DTT. Profilin was immobilized to Ni-matrix and the bound proteins were eluted with SumoStar protease cleavage of the His-tag. Input: actin source and His-tagged profilin, unbound: flow through of unbound proteins from Ni-matrix, protease elution: SumoStar elution of profilin and profilin bound actin, post-elution: imidazole elution of Ni- bound proteins.

We then subjected these profilactin complexes to gel filtration and compared their running profiles. The gel filtration was performed in the same buffer, where the complexes have been formed, but lacked EDTA and CytB. The difference between the actin isoforms became more obvious here. It was seen that almost all $\alpha$ actin dissociated from profilin during or before gel filtration and a large portion eluted from the column as high molecular weight assemblies (Figure 3-3). Whether these are true actin filaments or simply aggregates is not clear. On the other hand, profilactin formed with cytoplasmic actin remained stable during gel filtration. The free profilin eluting at the end of the column is not due to dissociation of the complex, but rather from incomplete saturation of the initial profilin in the binding reaction. With this, we could confirm the previous reports about the lower affinity of $\alpha$ actin towards profilin compared with $\beta / \gamma$ actin. This result also indicated that cytoplasmic actin is indeed a better substrate for the formation of an export complex in vitro. From this point on, we have used HeLa cytoplasmic extract as an actin source for complex formation assays. 

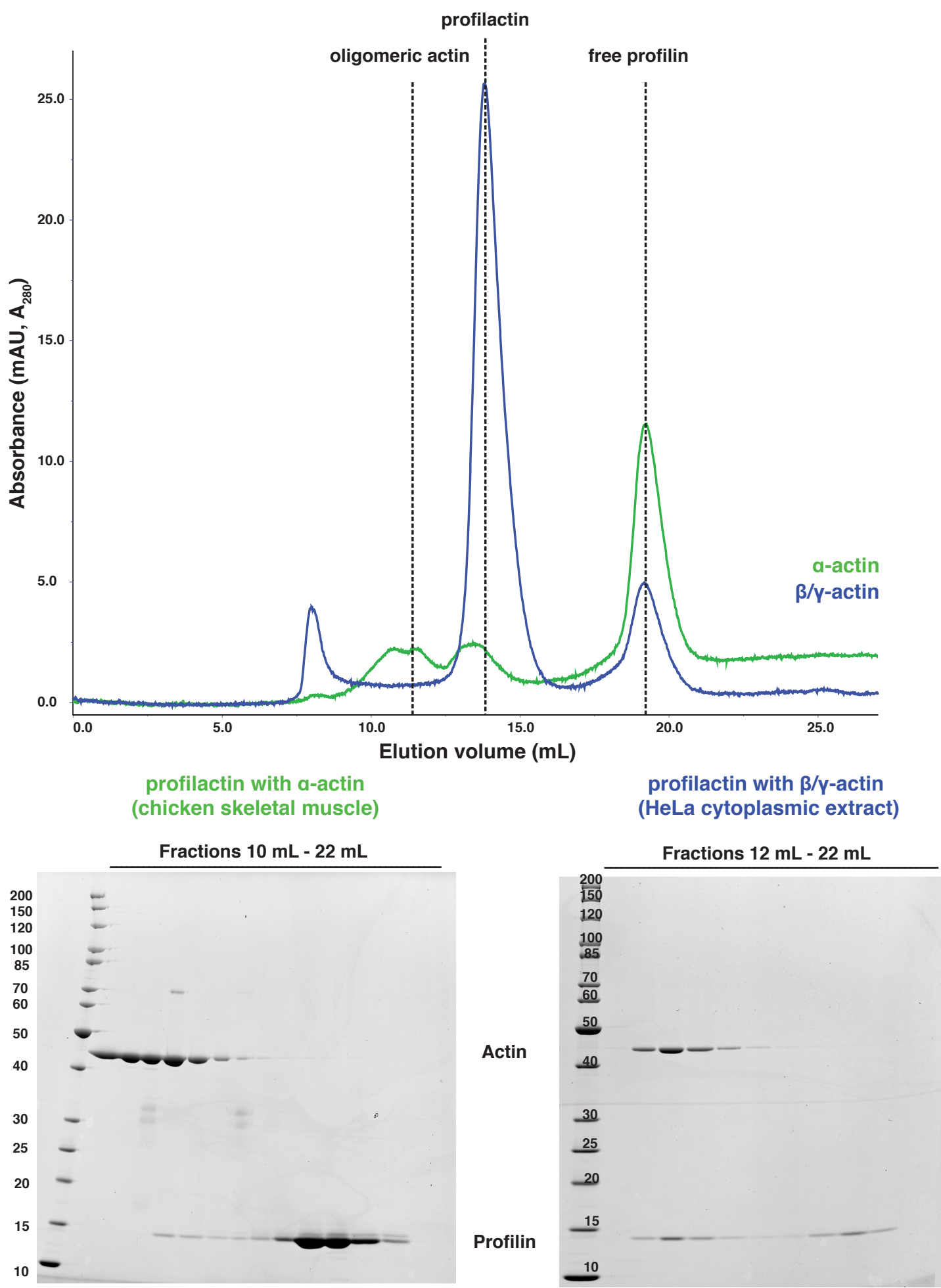

Figure 3-3 A stable profilactin complex can be formed with cytoplasmic actin

The upper panel shows the gel filtration chromatograms of profilactin complexes with $\alpha$ actin and cytoplasmic $(\beta / \gamma)$ actin on SD200 10/30 column equilibrated with $10 \mathrm{mM}$ Tris 7.5, $20 \mathrm{mM} \mathrm{NaCl}, 1 \mathrm{mM}$ DTT. Y-axis shows the UV absorbance at $280 \mathrm{~nm}, \mathrm{X}$-axis shows the elution volume. Green represents the chromatogram with $\alpha$ actin; blue represents the chromatogram with $\beta / \gamma$ actin. Lower panel shows the analysis of the gel filtration fractions via SDS PAGE. Left gel shows the fractions of the profilactin with $\alpha$ actin, right gel shows the fractions of the profilactin with $\beta / \gamma$ actin. 


\subsection{Screening for optimal conditions for the actin nuclear export complex}

In order to assemble a stable actin export complex, the optimal conditions have to be determined first. For screening a variety of buffer and salt conditions, we designed a phenyl sepharose (PS) based binding assay. Phenyl sepharose binds to NTRs with high affinity (Ribbeck and Gorlich, 2002), and the experimental setup for PS based binding assay is straightforward. We used purified, tag-free Xpo6 and RanGTP, and used HeLa cytoplasmic extract as a source for actin and profilin. The concentrations of the components were titrated to come to a saturation of Xpo6. In this assay, the components were mixed and diluted in a $500 \mu \mathrm{L}$ binding reaction with the buffer condition to be tested. PS matrix was included in the binding reaction for $3 \mathrm{~h}$, and after the removal of unbound material, bound proteins were eluted with SDS buffer. The specificity of complex formation on PS matrix was also confirmed with +/- Ran controls (Figure 3-4). While screening for the effects of salt on the complex formation, we found that with increasing salt concentration, less profilactin was bound to Xpo6. At $300 \mathrm{mM} \mathrm{NaCl}$ concentration, the loss of binding became more drastic.
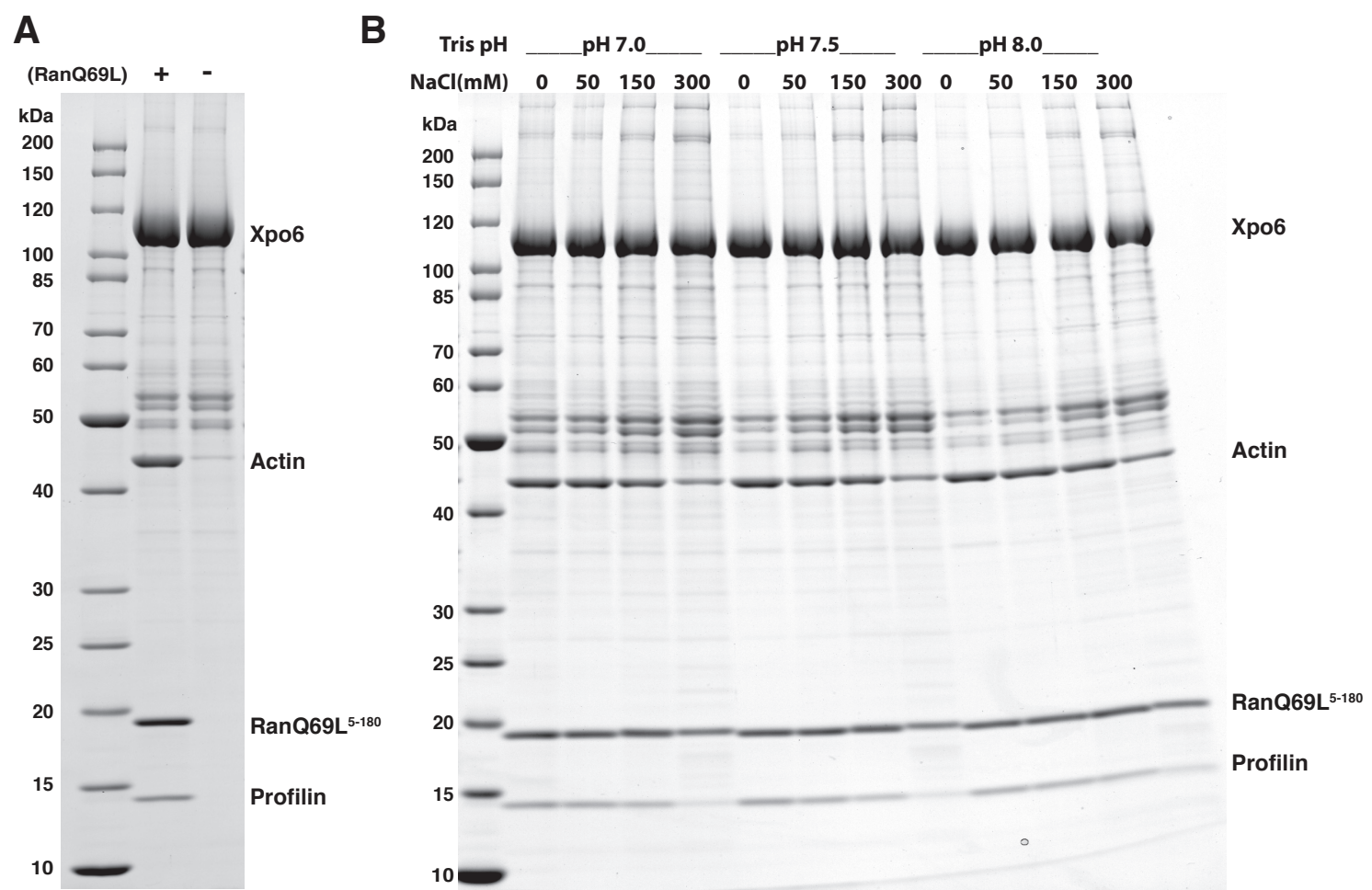

Figure 3-4 Effect of salt concentration on complex formation

$50 \mu \mathrm{L}$ HeLa S10 cytoplasmic extract was incubated with $0.25 \mathrm{nmol}$ tag-free Xpo6 and $0.5 \mathrm{nmol}$ tag-free RanGTP(5180)Q69L in indicated buffers. Xpo6 was immobilized to $10 \mu \mathrm{L}$ phenyl sepharose (PS) matrix. The unbound proteins were washed and the bound proteins were eluted with SDS buffer. A) Profilactin from HeLa cytoplasmic extract binds to Xpo6 in a RanGTP dependent manner. The SDS elution from the PS matrix is shown. We confirm the specificity of 
profilactin binding to Xpo6 on phenyl sepharose matrix. B). Binding conditions were varied between 0-300 $\mathrm{mM} \mathrm{NaCl}$ and Tris $\mathrm{pH}$ 7.0-8.0. The SDS elution from the PS matrix is shown.

Next, we screened for different buffers and $\mathrm{pH}$ for their effect on complex formation. We couldn't detect any preference for a certain buffer and pH (Figure 3-5). PS based complex formation was easy to perform, but the complex could only be removed from the matrix by denaturation. We therefore had to transfer the findings of PS experiments to another setup. We decided to proceed with low concentrations of buffer and salt because of our ultimate aim of crystallization.

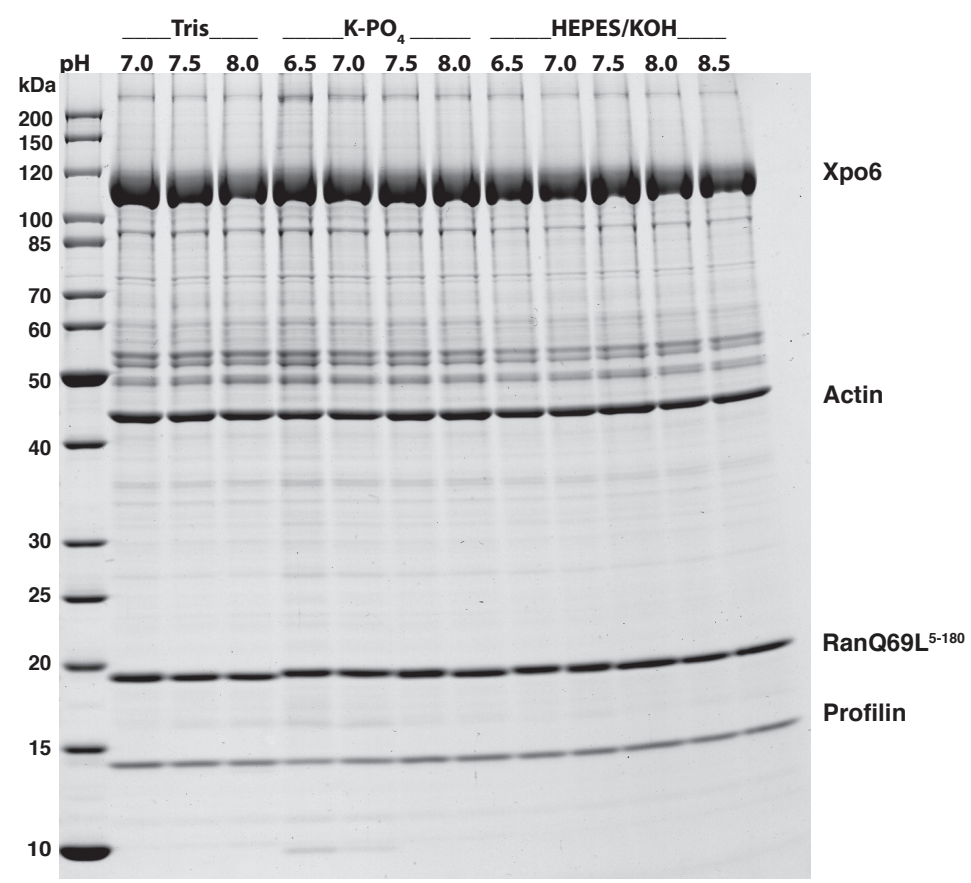

Figure 3-5 Effect of buffer ions and pH on complex formation

RanGTP dependent binding of profilactin to Xpo6 immobilized on phenyl sepharose matrix. Experimental setup was identical with Figure 3-4. Different buffer ions (Tris, HEPES, potassium phosphate $\left(\mathrm{K}_{-}-\mathrm{PO}_{4}\right), 10 \mathrm{mM}$ each) adjusted to various $\mathrm{pH}$ values were tested. $\mathrm{NaCl}$ was kept constant at $50 \mathrm{mM}$ for all experiments. Individual bands are labeled. No visible effect could be detected.

\subsection{Xpo6 and RanGTP can form a stable dimeric complex in vitro}

In our complex formation trials, we observed that RanGTP always migrated together with Xpo6 in gel filtration, even when actin was not incorporated. This is rather unusual for exportins, which usually have mediocre affinity for RanGTP in the absence of a cargo (Fornerod et al., 1997; Kutay et al., 1997). This brought up the question whether Xpo6 and Ran can form a stable dimer in the absence of cargo. We have used His tagged RanGTP and tag-free Xpo6 for complex formation. The complex was immobilized on Ni-matrix via the N-terminal His-tag of RanGTP and eluted with protease digestion of the tag. Xpo6 and RanGTP formed a complex in high ionic strength buffer $(500 \mathrm{mM} \mathrm{NaCl})$, and the complex 
remained stable as well in low salt buffer $(10 \mathrm{mM}$ Tris 7.0, $10 \mathrm{mM} \mathrm{NaCl}, 2 \mathrm{mM}$ DTT) used in gel filtration (Figure 3-6). This also showed that RanGTP remains stable in low salt concentrations, when it is bound by Xpo6.

\section{A}

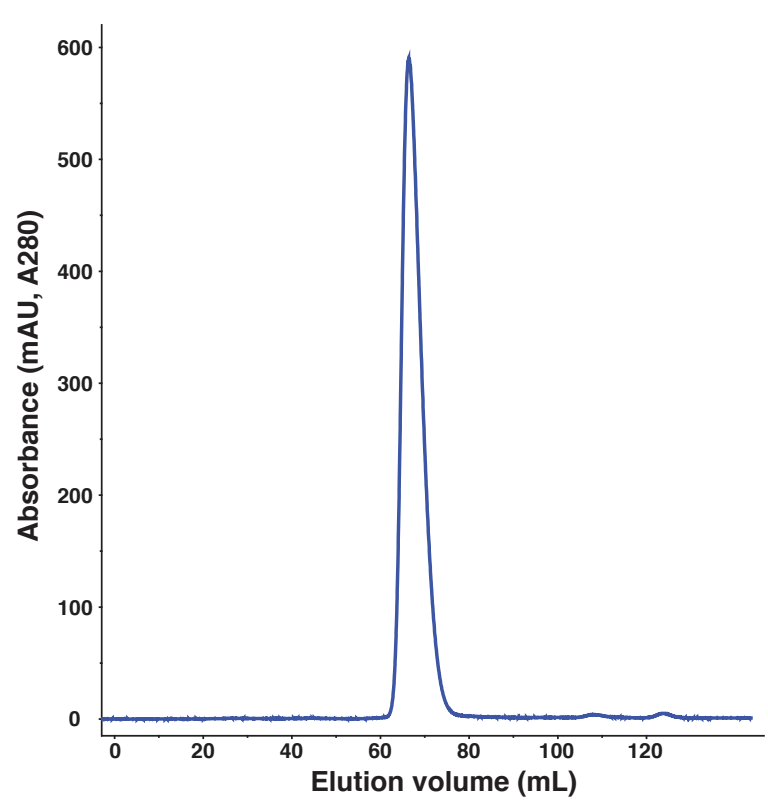

B input Fractions $62-72 \mathrm{~mL} \quad$ Standard

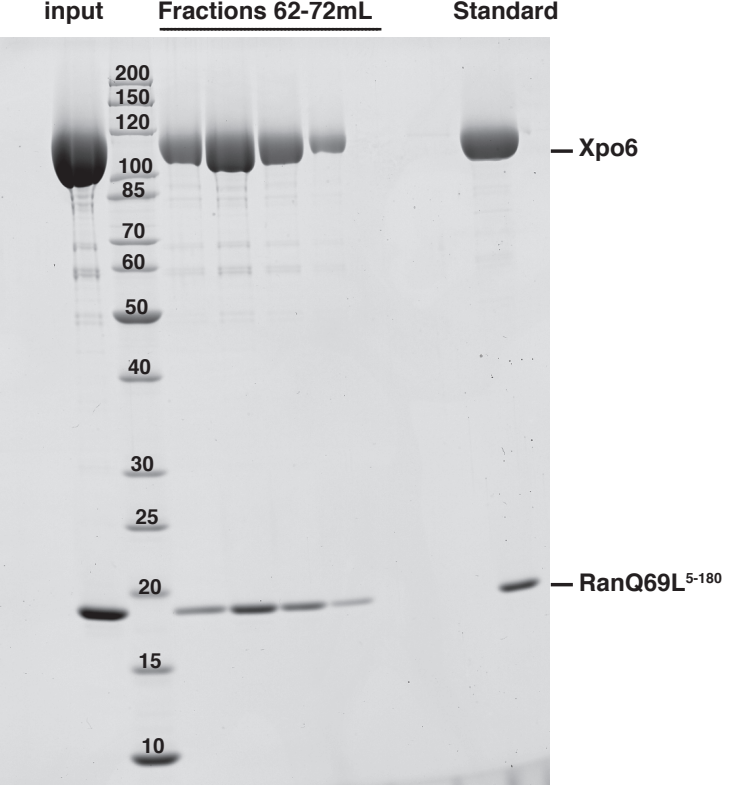

Figure 3-6 Xpo6 and RanGTP can form a stable complex in vitro

200 nmol His-tagged RanGTP(5-180) and tag-free Xpo6 were incubated and immobilized on Ni-matrix. The bound fraction was eluted from the column by Sumo cleavage of the His-tag of RanGTP. This fraction was concentrated and further purified with gel filtration. A) The size exclusion chromatogram for Xpo6•RanGTP complex on SD200 16/60 column equilibrated with $10 \mathrm{mM}$ Tris 7.0, $10 \mathrm{mM} \mathrm{NaCl}, 2 \mathrm{mM}$ DTT. Y-axis shows the UV absorbance at 280nm, X-axis shows the elution volume. B) Fractions of the indicated range from gel filtration in (A) on a gradient SDS polyacrylamide gel. Standard indicates a manually mixed solution of Xpo6 and RanGTP in 1:1 molar ratio. It was loaded for stoichiometry comparison with the peak fractions.

\subsection{Actin export complex can be formed via two stable sub- complexes}

We have used the approach to form the tetrameric export complex via two sub-complexes: profilactin and Xpo6•RanGTP. We have previously shown that these sub-complexes were stable in gel filtration in low salt buffers. This approach enabled us to overcome the discrepancy between the buffer conditions required for free actin and free RanGTP. Free actin tends to polymerize in high salt conditions, whereas free RanGTP precipitates in low salt conditions. As shown above, RanGTP forms a dimeric complex with Xpo6, which remains stable in salt concentrations as low as $10 \mathrm{mM}$. We have incubated pre-formed complexes of profilactin and Xpo6•RanGTP in this low salt buffer overnight in cold room. We have added the profilactin complex in excess, in order to be able to saturate the Xpo6•RanGTP. We then subjected this mixture to gel filtration. As seen in Figure 3-7, a 
tetrameric complex can be formed with this method. However, the excess profilactin in the mixture can not be separated from the export complex properly. There is also minor dissociation in profilactin such that free actin (as high molecular weight assemblies) and free profilin is seen in the chromatogram. For better purity, we took the peak fraction of the first gel filtration and subjected to gel filtration once more. We obtained a single peak and the analysis of the fractions showed that a stoichiometric complex could be formed with this method.

A

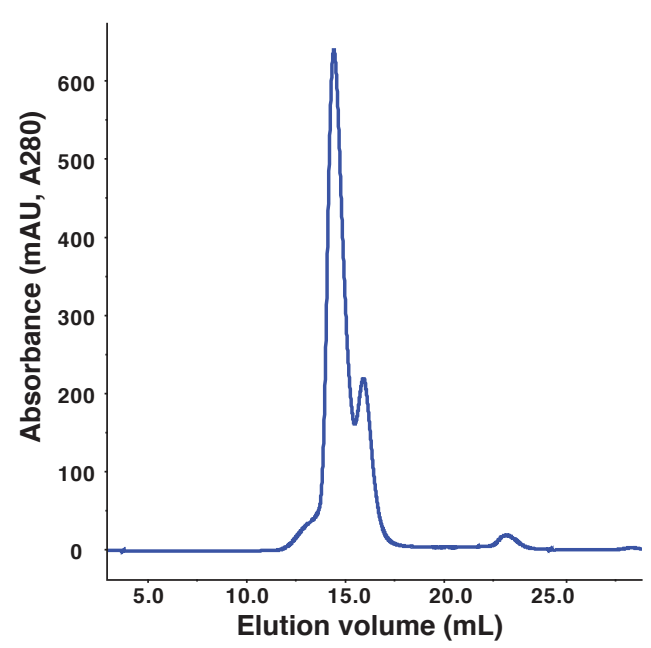

C

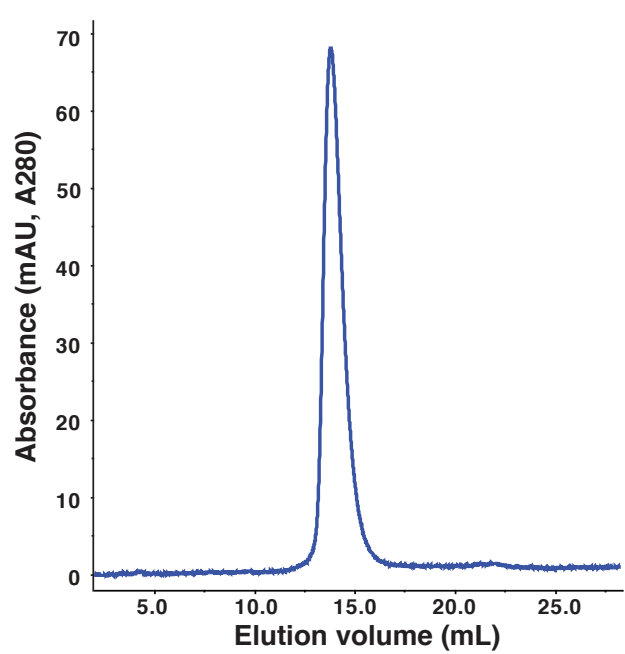

B

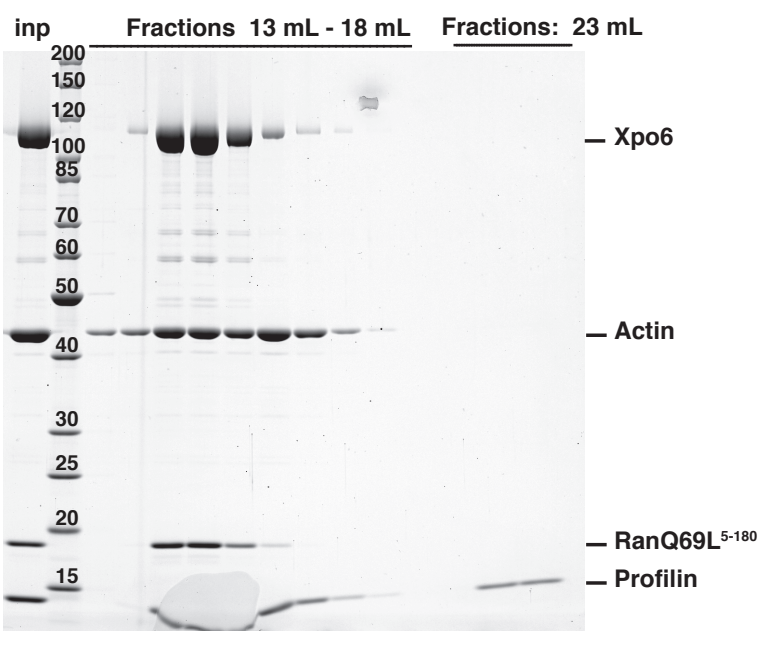

D

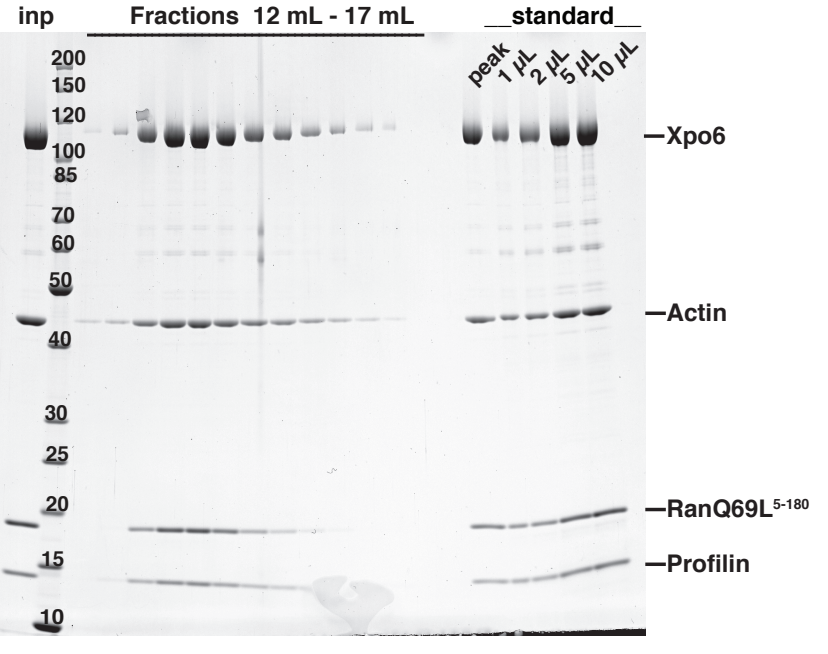

Figure 3-7 Formation of the actin nuclear export complex

Profilactin and Xpo6•RanGTP complexes in $10 \mathrm{mM}$ Tris $7.0,10 \mathrm{mM} \mathrm{NaCl}, 2 \mathrm{mM}$ DTT were mixed in near stoichiometric ratios (profilactin in excess) and incubated overnight in the cold room. The mixture was concentrated and applied to SD200 10/30 column equilibrated with the same buffer. The peak fractions of the first chromatogram were taken, concentrated and subjected to gel filtration again. A) The size exclusion chromatogram for the profilactin and Xpo6•RanGTP mix on SD200 10/30 column. Y-axis shows the UV absorbance at $280 \mathrm{~nm}, \mathrm{X}$-axis shows the elution volume. B) Fractions of the indicated range from gel filtration in (A) on a gradient SDS polyacrylamide gel. C) The size exclusion chromatogram for the peak fraction from B ( $3^{\text {rd }}$ and $4^{\text {th }}$ fractions in the gel) on SD200 10/30 column. Y-axis shows the UV absorbance at $280 \mathrm{~nm}, \mathrm{X}$-axis shows the elution volume. D) Fractions of the indicated range from gel filtration in (C) on a gradient SDS polyacrylamide gel. Standard indicates a manually mixed solution of Xpo6, RanGTP, $\alpha$ actin and profilin in equimolar ratio. It was loaded in varying amounts for stoichiometry comparison with the peak fraction. See text for experimental details. 


\subsection{Actin nuclear export complex is sensitive to ionic strength}

We wanted to test how much salt the actin export complex can tolerate. The PS experiments indicated that $\mathrm{NaCl}$ concentrations of $300 \mathrm{mM}$ significantly impair complex formation. The salt sensitivity can be a problem for crystallization screens, since most crystallization conditions contain salts. Export complex was formed via two subcomplexes in $10 \mathrm{mM}$ Tris 7.0, $10 \mathrm{mM} \mathrm{NaCl}, 1 \mathrm{mM}$ DTT as described in section 3.4. The peak fraction from the first gel filtration was taken, concentrated and divided into two. One aliquot remained in the low salt buffer, whereas the other aliquot was brought to $100 \mathrm{mM}$ $\mathrm{NaCl}$ concentration. After $1 \mathrm{~h}$ incubation, the peak fractions were run on gel filtration in their respective buffer conditions. Figure 3-8 shows the results of the second gel filtration. The effects were striking. Increased salt concentration in one aliquot completely dissociated the pre-formed complex, such that two peaks with two sub-complexes were observed. In the control aliquot, complex remained mostly stable. There was a slight tailing of the peak in the gel filtration, which was partly due to the dissociation on the gel filtration and partly due to inhomogeneous peak fraction sampling of the first gel filtration.

The complete dissociation of the complex at $100 \mathrm{mM} \mathrm{NaCl}$ was unexpected, considering the results of the phenyl sepharose experiments. In order to confirm the new findings, we designed a binding experiment on Ni-matrix. Either His-tagged profilin or His-tagged Xpo6 was used for the immobilization of the complex and different ionic conditions were tested. HeLa lysate and profilin were pre-mixed and incubated, to which pre-mixed Xpo6 and RanGTP were added. $50 \mu \mathrm{L}$ HeLa cytoplasmic extract, $1 \mu \mathrm{M}$ profilin (tagged or untagged), $1 \mu \mathrm{M}$ Xpo6 (tagged or untagged) and 1.2 $\mu \mathrm{M}$ RanGTP were used in a $500 \mu \mathrm{L}$ binding reaction. Low and high salt conditions (10 to $100 \mathrm{mM} \mathrm{NaCl})$ were prepared in 10 and $50 \mathrm{mM}$ Tris buffer $\mathrm{pH}$ 7.5. The immobilized and bound proteins were eluted with protease cleavage of the $\mathrm{N}$-terminal tags.

The results (Figure 3-9) confirmed the complex dissociation on gel filtration, and were dramatically different than PS experiments. The presence of $100 \mathrm{mM} \mathrm{NaCl}$ almost completely abolished complex formation. Not only $\mathrm{NaCl}$, but also increasing buffer concentration affected complex formation negatively, as judged by the further loss of binding in $50 \mathrm{mM}$ Tris conditions. The results were identical for complexes immobilized on profilin and Xpo6. Another interesting observation was that not only Xpo6•RanGTP, 
but also profilactin sub-complex remained stable in the tried conditions, meaning that it is the interface of these two sub-complexes that is sensitive to ionic conditions.

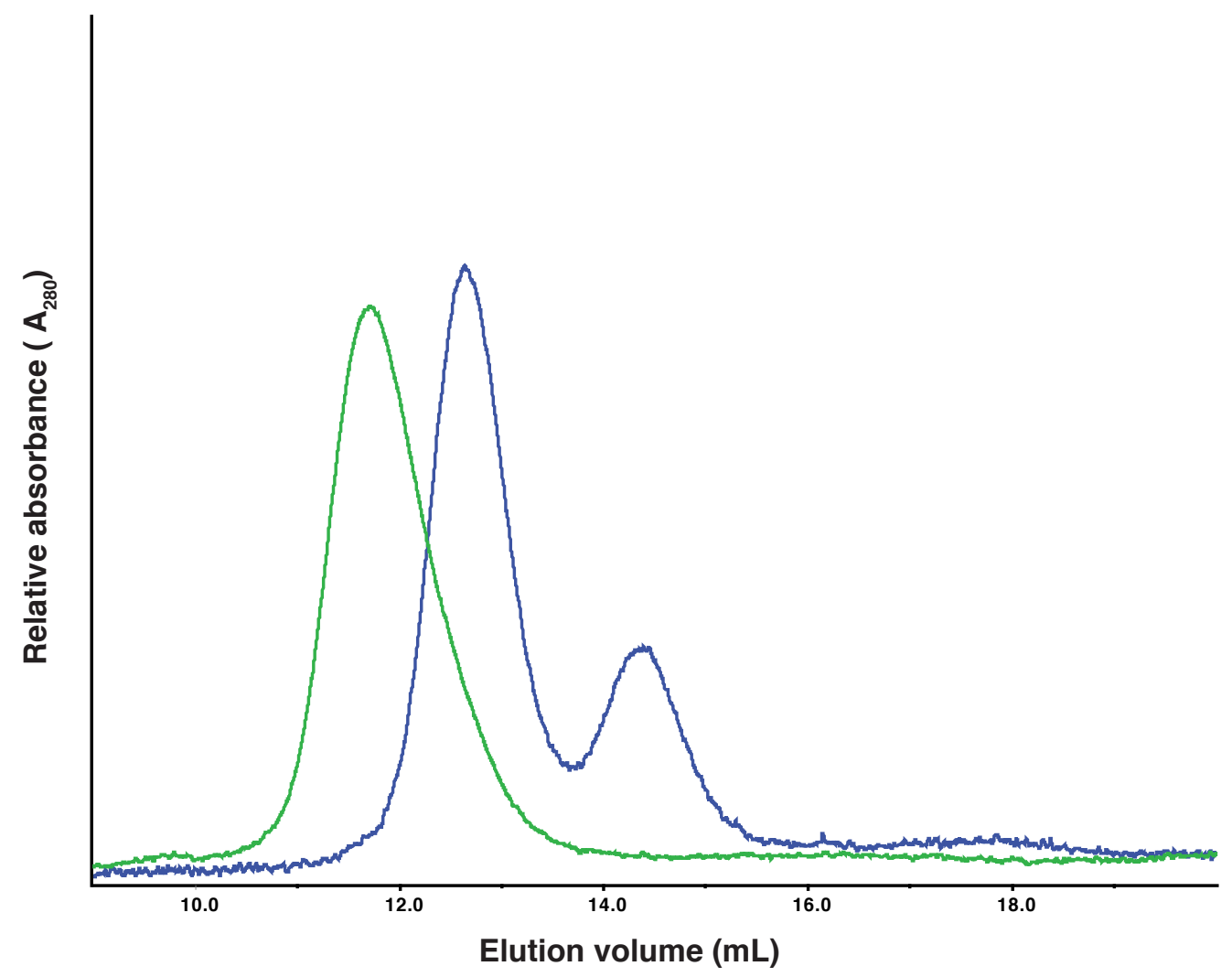

Complex in $10 \mathrm{mM} \mathrm{NaCl}$

Fractions $10.5 \mathrm{~mL}-13.5 \mathrm{~mL}$

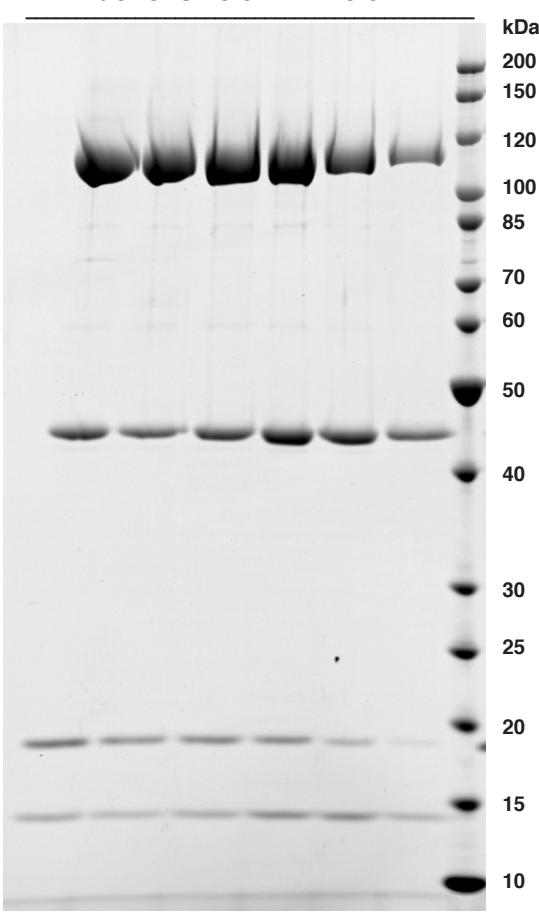

Complex in $100 \mathrm{mM} \mathrm{NaCl}$

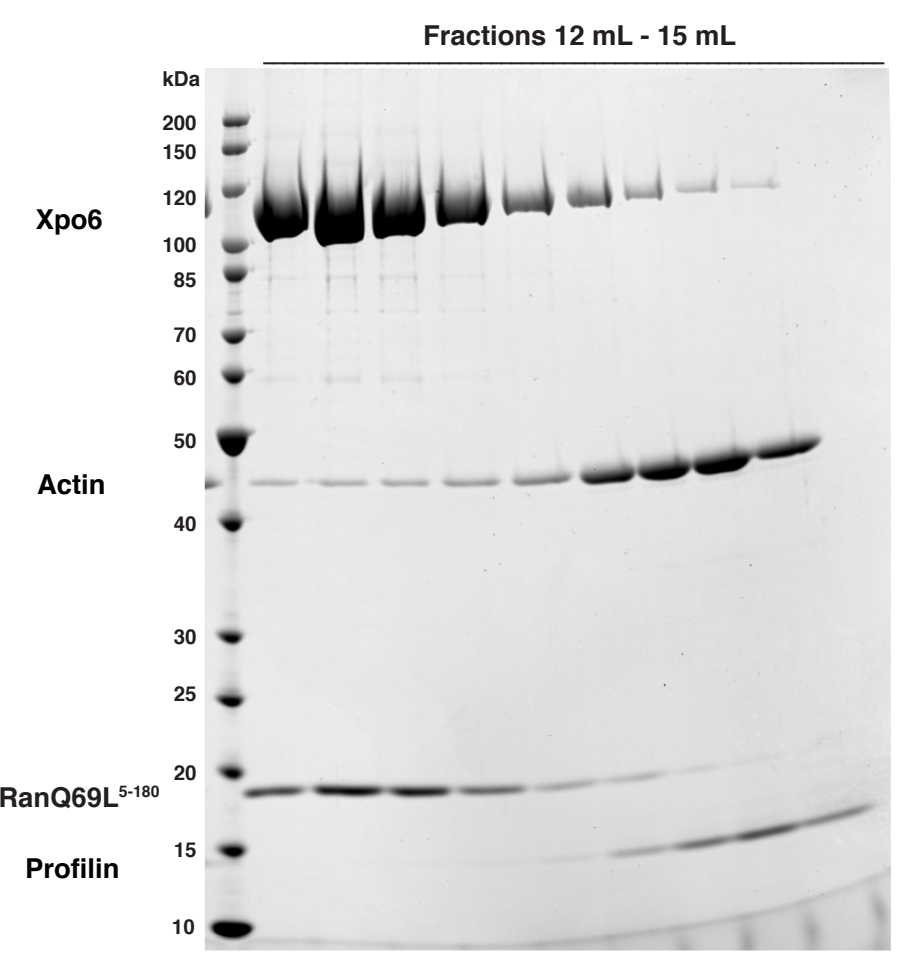

Figure 3-8 Increasing salt concentration dissociates a pre-formed actin export complex

Export complex was formed as described in Figure 3-7. The peak fraction of the first gel filtration was collected, split into two aliquots, and $\mathrm{NaCl}$ concentration in one aliquot has been increased to $100 \mathrm{mM}$. The aliquots were run on gel 
filtration equilibrated with their respective buffers after $1 \mathrm{~h}$ incubation. The upper panel shows the gel filtration chromatograms of actin export complexes incubated in different salt concentrations. Y-axis shows the UV absorbance at $280 \mathrm{~nm}, \mathrm{X}$-axis shows the elution volume. Green represents the chromatogram with low salt condition; blue represents the chromatogram with high salt condition. Lower panel shows the analysis of the gel filtration fractions via SDS PAGE. Left gel shows the fractions of the low salt complex, right gel shows the fractions of the high salt complex.

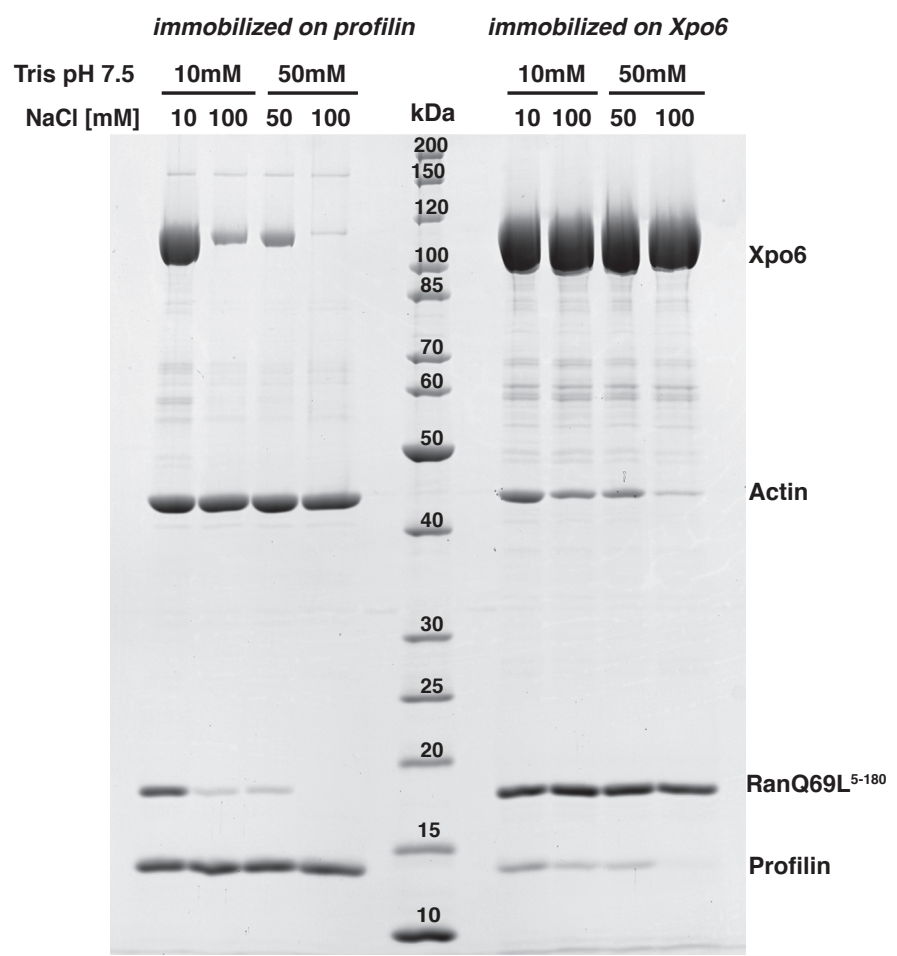

Figure 3-9 Formation of the actin nuclear export complex is salt sensitive

His-tagged Profilin or His-tagged Xpo6 were used to immobilize the export complex on Ni-matrix. $50 \mu \mathrm{L}$ HeLa extract, $0.5 \mathrm{nmol}$ Profilin $0.5 \mathrm{nmol}$ Xpo6 and $0.6 \mathrm{nmol}$ RanGTP were used. Varying $\mathrm{NaCl}$ (10 to $100 \mathrm{mM}$ ) and buffer (Tris $\mathrm{pH}$ $7.510-50 \mathrm{mM}$ ) concentrations were used during binding, to test the effect on complex formation. The complexes were bound to Ni-matrix via the N-terminal tags of profilin or Xpo6, which were eluted by the SumoStar cleavage of the Nterminal tags. The lanes on the left show the SumoStar elution of the complexes immobilized on profilin, the lanes on the left on Xpo6. Individual protein bands are labeled.

\subsubsection{The ionic species in the solution affect the export complex formation}

Latest results indicated that phenyl sepharose might be stabilizing the complex and masking the effect of salt on the complex formation. To be able to screen for optimal conditions for the complex formation, we performed the salt and buffer screens on Nimatrix. $0.5 \mu \mathrm{M}$ Profilin, $50 \mu \mathrm{L}$ HeLa cytoplasmic extract, $1 \mu \mathrm{M}$ Xpo6 and $1.2 \mu \mathrm{M}$ RanGTP were used in a $500 \mu \mathrm{L}$ binding reaction. Profilactin was formed and immobilized on Ni-matrix in a low salt buffer $(10 \mathrm{mM}$ Tris $\mathrm{pH} 7.5,10 \mathrm{mM} \mathrm{NaCl}, 1 \mathrm{mM}$ DTT). The unbound proteins were removed. The pre-incubated Xpo6•RanGTP was added to the matrix in $500 \mu \mathrm{L}$ of the buffer condition to be tested. After $3 \mathrm{~h}$ incubation, the unbound proteins were removed, and the complex was eluted from the matrix by protease cleavage of profilin's N-terminal tag. Low $(10 \mathrm{mM})$ and slightly higher $(50 \mathrm{mM})$ concentrations of $\mathrm{NaCl}, \mathrm{KCl}, \mathrm{CH}_{3} \mathrm{COONa}$ (Sodium acetate, abbreviated as $\mathrm{NaAc}$ ), and $\mathrm{CH}_{3} \mathrm{COOK}$ 
(potassium acetate, abbreviated as KAc) salts were tested. The "high" salt concentration was kept at $50 \mathrm{mM}$ in order to be able to detect subtle differences. The buffer was kept constant at $10 \mathrm{mM}$ Tris $\mathrm{pH}$ 7.5. It was also tested whether the addition of glycerol (5\%) improved the complex stability. A slight reduction in the bound Xpo6 and RanGTP amount was detected for $50 \mathrm{mM}$ salt conditions for $\mathrm{NaCl}, \mathrm{KCl}$ and $\mathrm{KAc}$. For $\mathrm{NaAc}$, this loss of binding was not visible. No difference was observed upon addition of glycerol (Figure 3-10, A).

A

\section{B}

$\mathrm{NaCl}$ $\frac{\mathrm{KCl}}{10 \mathrm{mM} 50}$ $\frac{\mathrm{NaAc}}{10 \mathrm{mM}+50 \mathrm{mM}}$ $\frac{\mathrm{KAC}}{10 \mathrm{mM} 50 \mathrm{mM}}$ Gly $5 \% \frac{10 \mathrm{mM}}{-+\frac{50 \mathrm{mM}}{-++}}$ $\mathrm{Da}-+-$ $++-+$

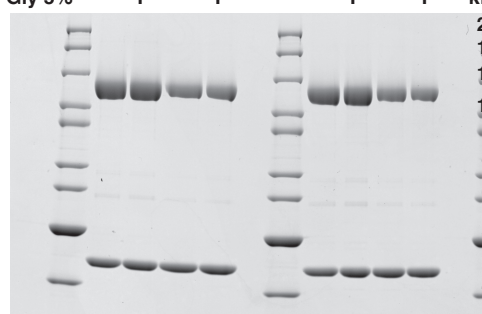

200 150 a 85 $\frac{70}{60}$
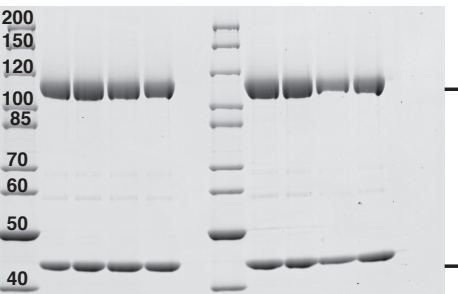

2mM MgAc

$\mathrm{NaCl}$ $10 \mathrm{mM} 100 \mathrm{mM} \quad 10 \mathrm{mM} \quad 100 \mathrm{mM}$ 200
150 Xpo6 - $\cup-120$
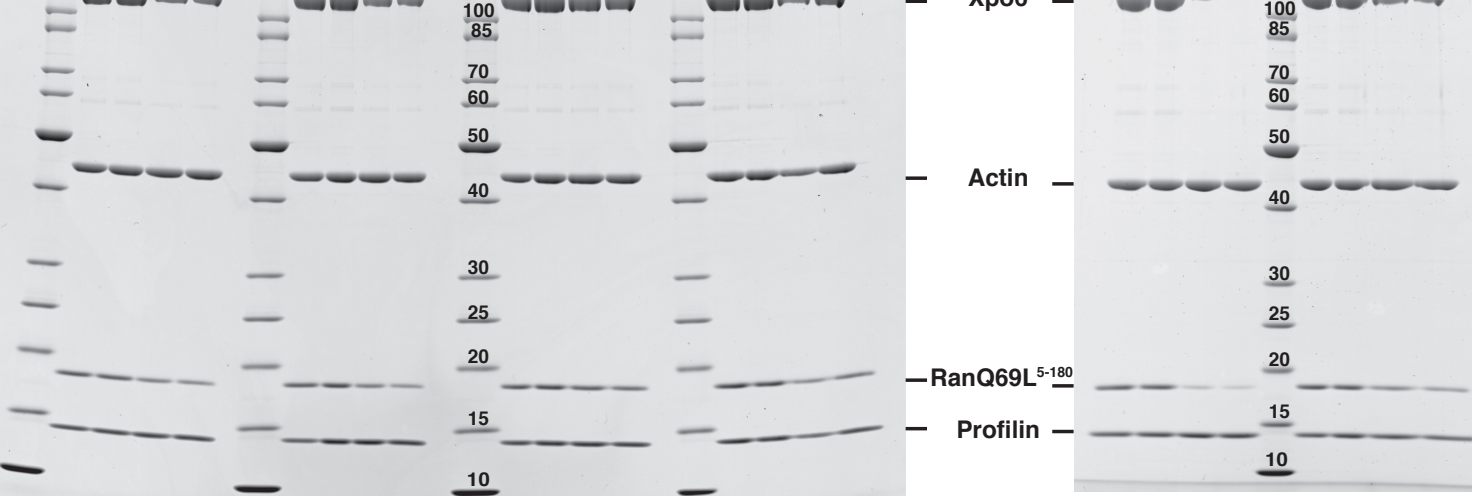

Figure 3-10 The ionic species in the buffer have an effect on the complex formation

Experimental setup was same as Figure 3-9. Here, the complex was immobilized on Ni-matrix via the N-terminal Histag of profilin. Salt conditions were varied to test their effect on complex formation. A) sodium chloride (NaCl), potassium chloride $(\mathrm{KCl})$, sodium acetate $(\mathrm{NaAc})$ and potassium acetate (KAc) salts were used at $10 \mathrm{mM}$ and $50 \mathrm{mM}$ concentrations. +/- 5\% Glycerol was tested for each condition. $10 \mathrm{mM}$ Tris pH 7.5 was kept constant for all reactions. The amount of bound Xpo6•RanGTP indicated the stability of the complex in that condition. B) Effects of sodium chloride and sodium acetate on complex formation were compared at $10 \mathrm{mM}$ and $50 \mathrm{mM}$ concentrations. $+/-5 \%$ Glycerol was tested for each condition. $10 \mathrm{mM}$ Tris $\mathrm{pH} 7.5$ was kept constant for all reactions.

Since the difference between $\mathrm{NaAc}$ and other salts was not very dramatic, another binding assay was performed with higher salt concentrations. $\mathrm{NaCl}$ and $\mathrm{NaAc}$ salts were compared at 10 and $100 \mathrm{mM}$ concentrations, while $10 \mathrm{mM}$ Tris $\mathrm{pH} 7.5$ was kept constant. In parallel, the addition of $2 \mathrm{mM} \mathrm{Mg}\left(\mathrm{C}_{2} \mathrm{H}_{3} \mathrm{O}_{2}\right)_{2}$ (Magnesium acetate, abbreviated $\mathrm{MgAc}$ ) was tested for its effects on complex formation. The binding assay was performed as described above. The differences between $\mathrm{NaCl}$ and $\mathrm{NaAc}$ were more obvious in this setup (Figure 3-10, B). At $100 \mathrm{mM}$ concentration, $\mathrm{NaCl}$ almost completely inhibited complex formation. At 100 $\mathrm{mM} \mathrm{NaAc}$, the binding was impaired as well, but the loss of binding was about $50 \%$. It was obvious that $\mathrm{NaAc}$ was better tolerated than $\mathrm{NaCl}$ by the actin export complex. No significant effect of $\mathrm{MgAc}$ was observed, but we decided to use it in the complex 
formation, because both actin and RanGTP bind divalent cations $\left(\mathrm{Mg}^{++}\right)$to stabilize their nucleotide.

Different buffers in varying $\mathrm{pH}$ values were tested on PS matrix, and no effect was observed. We repeated this experiment on Ni-matrix (as described above) and obtained results that were different than the initial ones (Figure 3-11). The experiment showed the variation in $\mathrm{pH}$ was well tolerated in Tris and HEPES $(/ \mathrm{KOH})$ buffers. But the complex was less stable in potassium phosphate $\left(\mathrm{K}-\mathrm{PO}_{4}\right)$ buffer and the stability showed a strong dependency on the $\mathrm{pH}$. In $\mathrm{K}_{-} \mathrm{PO}_{4} \mathrm{pH} 6.5$ the binding of Xpo6 and RanGTP to profilactin was the weakest, and it gradually increased with the increasing $\mathrm{pH}$. This was an interesting observation since the ionic strength of a $\mathrm{pH} 8.0 \mathrm{~K}-\mathrm{PO}_{4}$ buffer is higher than that of a $\mathrm{pH}$ 6.5 $\mathrm{K}_{-}-\mathrm{PO}_{4}$ buffer. For further experiments we decided to continue using low concentrations of Tris $\mathrm{pH} 7.5$ buffer.

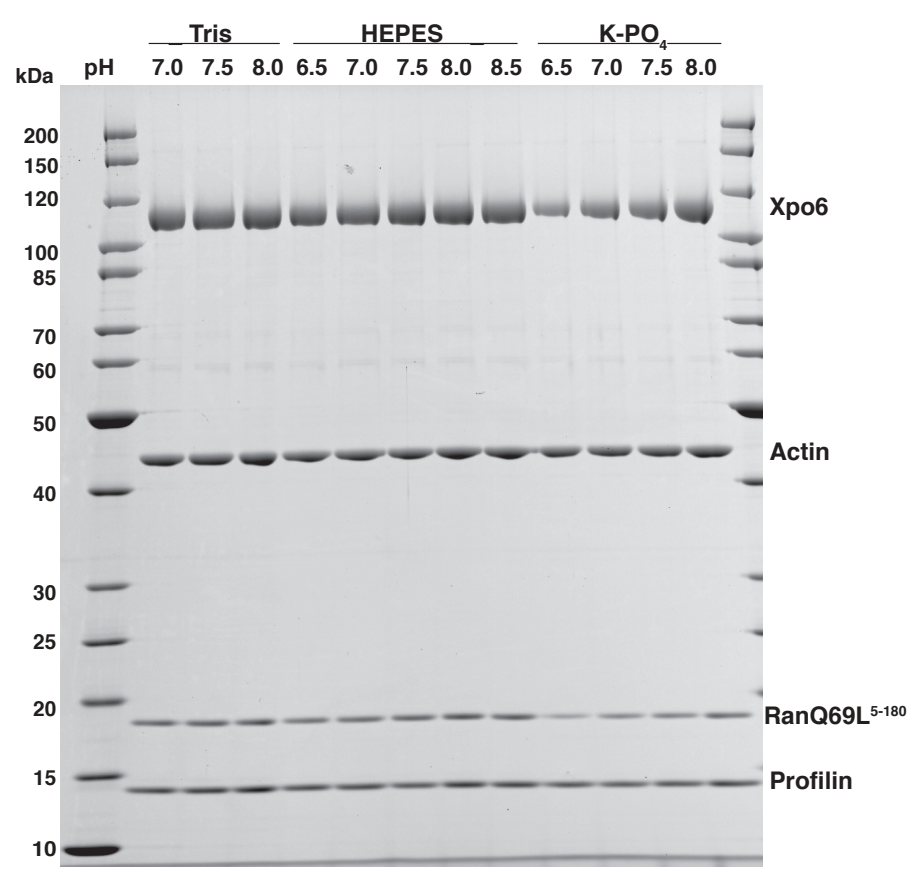

Figure 3-11 pH and buffer ions have an effect on complex formation

The effect of different buffer ions and $\mathrm{pH}$ on complex formation was tested in a binding assay. Experimental setup was identical as in Figure 3-10. $10 \mathrm{mM}$ of Tris, HEPES, and potassium phosphate $\left(\mathrm{K}-\mathrm{PO}_{4}\right)$ adjusted to the indicated pH values were tested. NaAc was kept constant at $50 \mathrm{mM}$ for all experiments. Individual bands are labeled. Complex stability in the tested condition was judged by the amount of $\mathrm{Xpo} 6 \bullet \mathrm{RanGTP}$ bound to profilactin. In $\mathrm{K}_{-} \mathrm{PO}{ }_{4}$ buffers, the complex was less stable and the effect was $\mathrm{pH}$ dependent 


\subsection{Improving the strategy for the actin nuclear export complex formation}

We showed that the actin export complex could be formed via two sub-complexes in low salt conditions. However, the protocol was very long and cumbersome, including 4 gel filtration steps: one for each of the sub-complexes and two consecutive gel filtrations for the tetrameric complex. We therefore used a simpler protocol for the preparative scale purifications of the actin export complex, which were used later for the crystallization trials. This protocol was also employed in small scale for the binding assays: His-tagged profilin was incubated with HeLa cytoplasmic extract and immobilized on Ni-matrix. After washing the unbound material, pre-incubated Xpo6 and RanGTP were added to the matrix in excess, in low salt conditions, instead of the elution of profilactin. This second incubation allowed the formation of a tetrameric complex, which was still immobilized on the Ni-matrix. The unbound proteins were removed and the export complex was eluted with protease cleavage of the N-terminal tag of profilin. We could scale up this protocol for preparative purification of the complex for crystallization. Figure 3-12 shows the preparation steps of the export complex. We optimized our "complex buffer" relying on the previous experiments: $10 \mathrm{mM}$ Tris $\mathrm{pH} 7.5,20 \mathrm{mM} \mathrm{NaAc}$ (pH adjusted to 7.5), $2 \mathrm{mM}$ MgAc, $1 \mathrm{mM}$ DTT. 5mM imidazole was added to the buffers to reduce unspecific binding to Ni-matrix. We have seen that more Xpo6•RanGTP was bound to profilactin with longer incubation times. So for the large-scale preparation we have incubated Xpo6 $\bullet$ RanGTP over night in cold room with profilactin bound to Ni-matrix. The complex was eluted from the matrix with protease cleavage of the N-terminal tag of profilin. The complex was concentrated and applied to a gel filtration column (SD200 16/60) equilibrated with the complex buffer. Figure 3-13 shows the gel filtration profile of the actin export complex and the SDS-PAGE analysis of the fractions. 


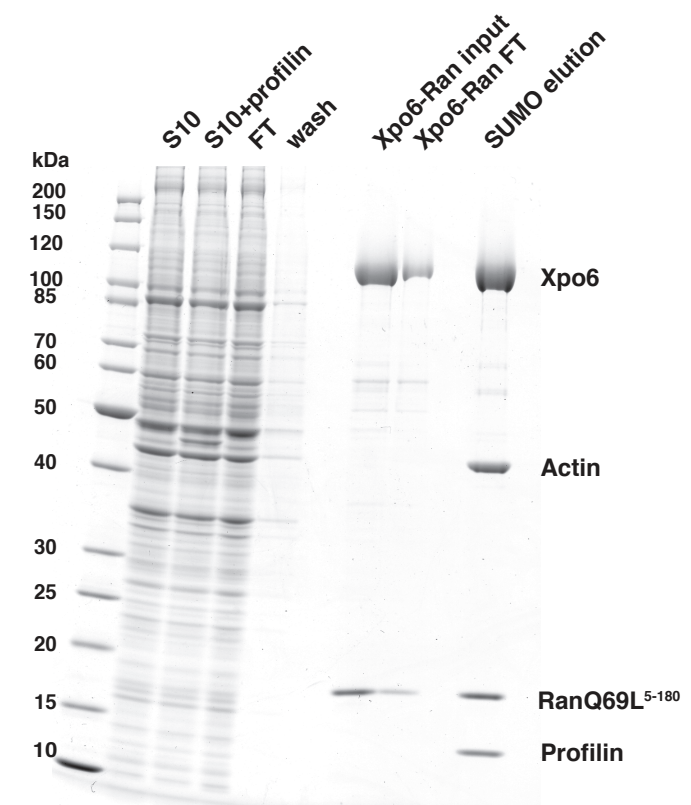

Figure 3-12 Purification of the actin nuclear export complex in large scale for crystallization

Purification steps of the human actin nuclear export complex. HeLa cytoplasmic extract (S10) was incubated with Nterminally tagged profilin, which is then immobilized to Ni-matrix. The unbound matrial was removed, and the matrix was washed with buffer to remove contaminants. Pre-incubated Xpo6 and RanGTP in excess over profilin were added to the matrix, and incubated overnight. The unbound proteins were removed. The complex was eluted by the cleavage of profilins N-terminal tag with SumoStar protease. Note that the complex purified with this strategy is already highly pure as judged by the SDS PAGE analysis.

A

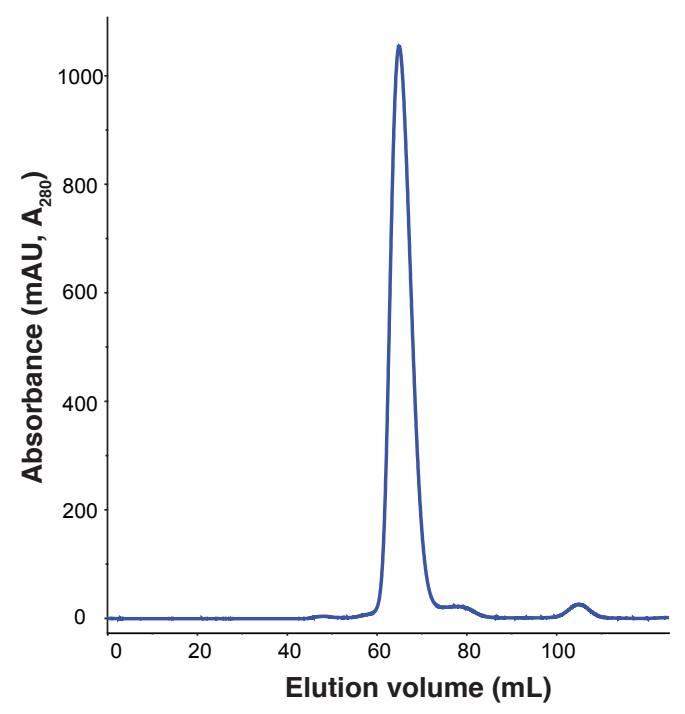

B

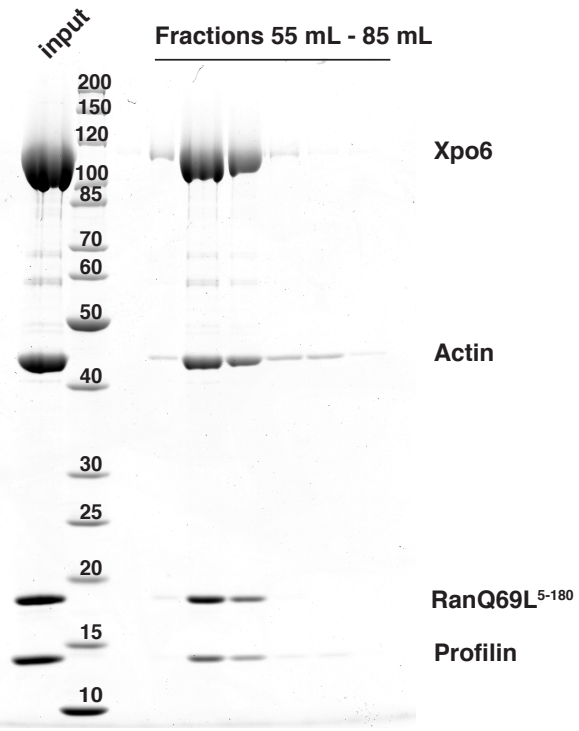

Figure 3-13 Gel filtration of the actin nuclear export complex

A) The size exclusion chromatogram for the actin export complex on SD200 16/60 column. Y-axis shows the UV absorbance at $280 \mathrm{~nm}, \mathrm{X}$-axis shows the elution volume. B) Fractions of the indicated range from gel filtration in (A) on a gradient SDS polyacrylamide gel. Second and third fractions were pooled and concentrated for crystallization experiments. 
As seen in the figure, the complex remains stable on the column and elutes as a symmetric peak, except for the shoulder to the right side of the peak. When the fractions were analyzed, it was seen that the shoulder consists of a small amount of free profilactin. This probably is a result of incomplete saturation of profilactin with Xpo6 $\bullet$ RanGTP, rather than dissociation on the column.

We have subjected the purified complex to multi angle light scattering (MALS) analysis. Using MALS one can measure the absolute molecular weight of particles. The principles of the method are described in (Wyatt, 1993) and briefly explained in 6.2.9. MALS is also employed for the determination of stoichiometry of protein complexes (Mogridge, 2004). Our MALS system is coupled to gel filtration, such that analysis can be restricted to individual peaks on the gel filtration. The purified complex eluted as a single peak with a tailing towards the right side (Figure 3-14). The analysis showed the average molecular weight of the indicated peak as $177 \mathrm{kDa}$. However, the expected molecular weight of the actin nuclear export complex is $205 \mathrm{kDa}(128 \mathrm{kDa}($ Xpo6) $+42 \mathrm{kDa}($ actin $)+20 \mathrm{kDa}$ (RanQ69L(5-180)) + $15 \mathrm{kDa}$ profilin). The difference between the expected and the calculated molecular weight is $28 \mathrm{kDa}$, which corresponds to the half of the molecular weight of profilactin $(57 \mathrm{kDa})$. This is an interesting finding and indicates that there might be a dissociation of the sub-complexes on the gel filtration, resulting in an inhomogeneous population where also cargo-free $\mathrm{Xpo6} \bullet \mathrm{RanGTP}$ is present.

A

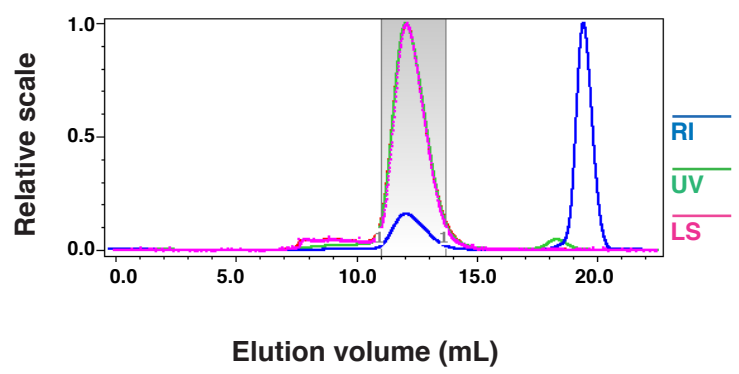

B

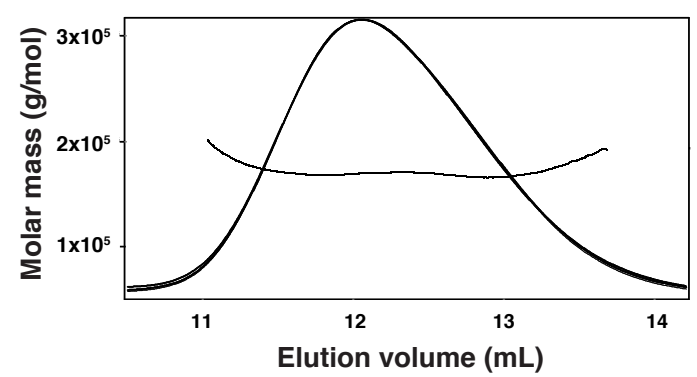

Figure 3-14 Molecular weight analysis of the actin nuclear export complex with MALS

A) The gel filtration profile of the actin export complex for MALS analysis. The peak area for molecular weight calculations is shaded gray. Concentration of the sample is measured by UV absorption (green) and refractive index (blue). Scattered light by the eluting particles is shown in pink. Note the tailing of the peak towards the right side. B) The distribution of the calculated molecular weights (average $177 \mathrm{kDa}$ ) within the selected peak area. A straight line indicates a homogenous mass distribution throughout the selected peak area. The curvature of the line possibly results from an inhomogeneous population migrating together in the peak area. 


\subsection{Topological analysis of the actin nuclear export complex}

We tried to derive the topology of the actin export complex by testing the accessibility of the cargo from different sites. Actin is very well mapped for its interactions with other actin binding proteins and small molecules (dos Remedios et al., 2003; Dominguez, 2004; Dominguez and Holmes, 2011). By analyzing the accessibility of certain binding sites on profilactin molecule, we can infer how Xpo6 might interact with profilactin. One site for testing the accessibility is the Poly-L-Proline binding cleft of profilin. This site is localized directly opposite to the actin-binding surface, in a groove created by the closely positioned N- and C-terminal helices of profilin (Figure 4-1 and (Ferron et al., 2007)). Most ABPs (cofilin, gelsolin, ciboulot, vitamin D binding protein, MAL) bind to the same site (the target binding cleft) of actin as profilin (Dominguez, 2004; Dominguez and Holmes, 2011), and are therefore mutually exclusive with profilin. These proteins can not be used as tools for testing the accessibility. DNaseI, however, binds to a specific loop in the subdomain 2 (Figure 2-6), which is distant from profilin binding site. Actually profilactin can efficiently bind DNaseI, and this was how profilin was identified in the first place (Carlsson et al., 1976). So, we have chosen the D-loop of actin as another site for accessibility testing.

We tested the accessibility of these sites by immobilizing the export complex to Poly-Pro sepharose matrix (prepared by Dirk Görlich) and DNaseI matrix (see section 6.2.5 for matrix preparation). As controls we also used free actin and profilin, as well as the profilactin and Xpo6•RanGTP complexes. For Poly-Pro matrix, profilin and profilactin served as positive controls, whereas free actin and Xpo6•RanGTP complex served as negative controls. On the other hand, free actin and profilactin should efficiently bind DNaseI matrix, where free profilin and Xpo6•RanGTP were negative controls. The proteins were incubated with both matrices for $2 \mathrm{~h}$ in cold room, and after washing the unbound material, the bound proteins were eluted by SDS buffer. Figure 3-15 shows the results of the accessibility assay.

As expected, free proline and profilactin bind to the Poly-Pro matrix, whereas free actin and Xpo6•RanGTP show only low levels of unspecific binding. The actin export complex was bound to Poly-Pro matrix in near stoichiometric ratios, which indicates that the PolyPro binding pocket of profilin is accessible within the export complex. Also, there is a certain level of contamination of the neighboring lanes with the export complex, due to the high concentration of protein in the loaded sample. As for the DNaseI matrix, it was seen 
that the positive controls (actin and profilactin) were bound to the matrix as expected. However, the negative control (Xpo6•RanGTP) was also bound to the matrix quite strongly, such that the results of the export complex binding could not be clearly interpreted. This result might be due to the insufficient quenching of the matrix after DNaseI coupling.

Therefore, we decided to test the accessibility of the actin export complex with a reciprocal binding experiment. In this setup we immobilized actin export complex to Ni-matrix as described in section 3.5.1, and before the elution of the complex with the tag cleavage, we performed an additional $1 \mathrm{~h}$ incubation with DNaseI. In the control sample, we incubated the immobilized complex with buffer for $1 \mathrm{~h}$ before the elution. As seen in Figure 3-16, incubation with DNaseI for 1 hour efficiently dissociates most of the Xpo6 $\bullet$ RanGTP form profilactin. This result indicates that the binding of DNaseI to actin is not compatible with its binding to the export complex.
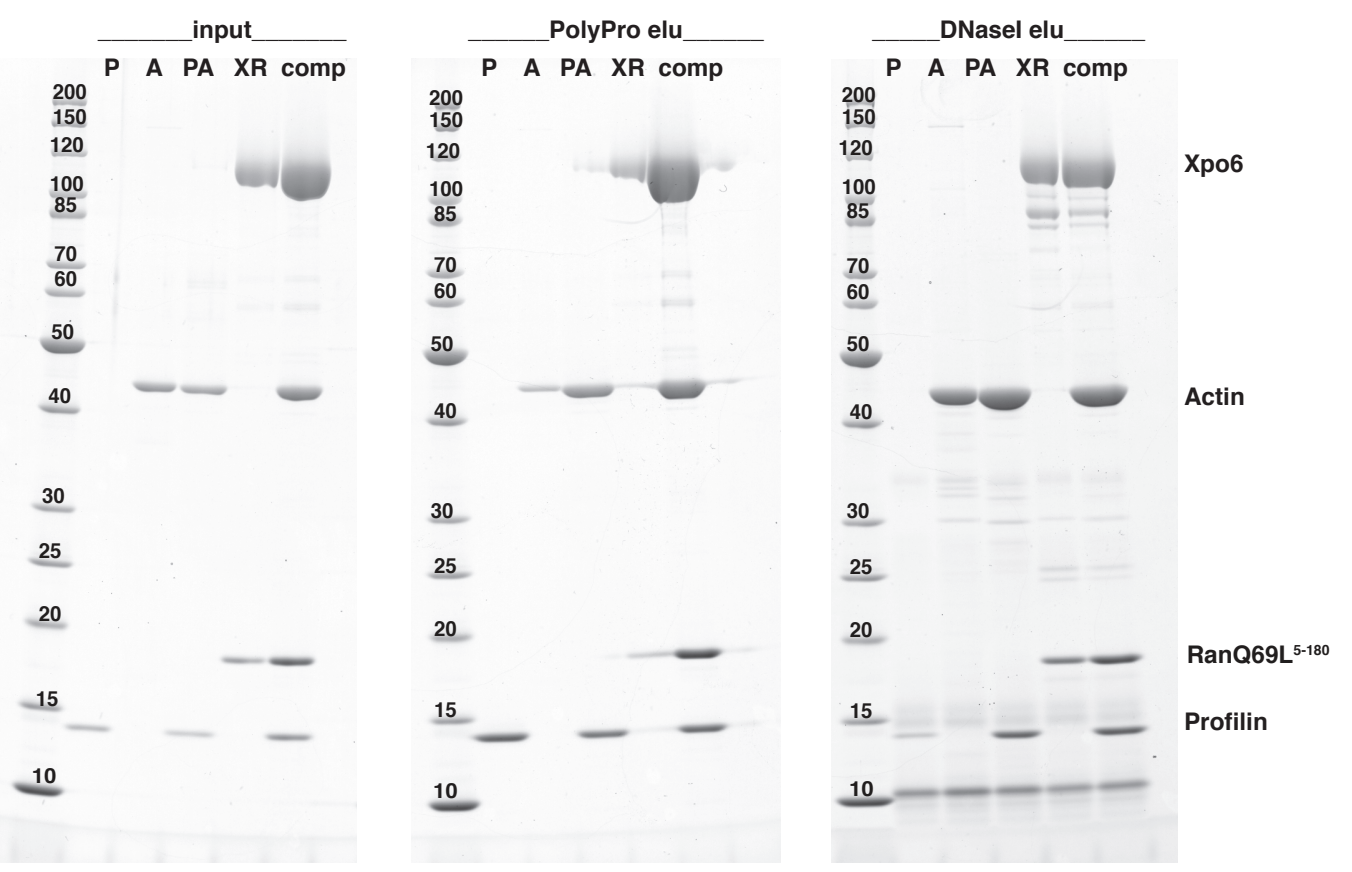

Figure 3-15 Accessibility of profilactin in the export complex

Equal and saturating amounts $(1.5 \mu \mathrm{M})$ of Profilin (P), actin (A), profilactin complex (PA), Xpo6•RanGTP complex (XR) and the actin export complex (comp) were incubated with either Poly-L-Pro or DNaseI matrix to test the accessibility of Poly-Pro binding pocket of profilin and the D-loop of actin in the export complex, respectively. Elution was performed with SDS buffer. Elution fractions were 10 times concentrated as the input fraction. Note that the complete export complex can be trapped on Poly-Pro matrix, showing the accessibility of the Poly-Pro binding pocket. In DNaseI matrix, the negative control (XR) showed unspecific binding to the matrix, so that the results could not be interpreted unambiguously. 


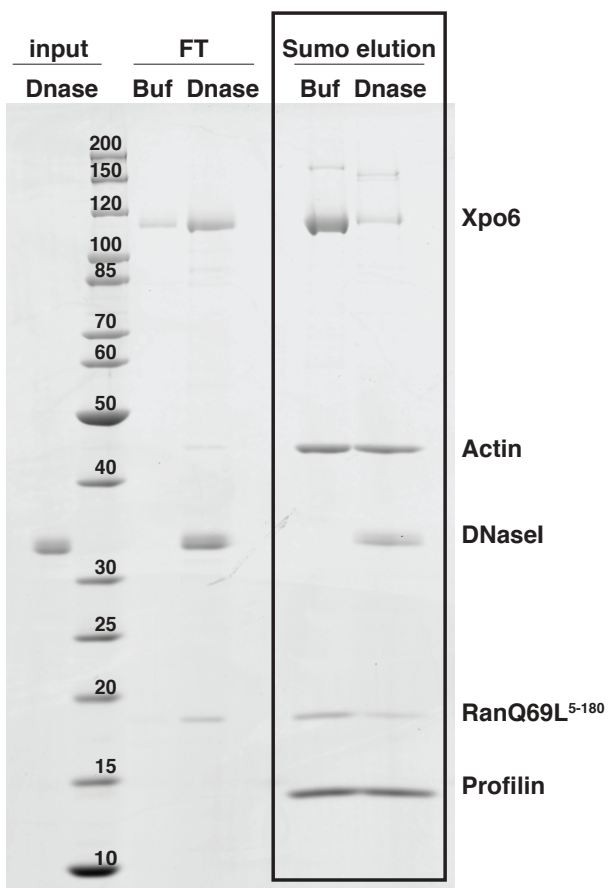

Figure 3-16 DNaseI can dissociate a pre-formed actin nuclear export complex

The export complex immobilized on Ni- matrix via the N-terminal tag of Profilin was incubated for 1 h with DNaseI (in excess) or with buffer. The input (DNaseI), Flow through and the SUMO elution fractions were analyzed on SDS PAGE. Note that DNaseI efficiently displaced a large fraction of Xpo6•RanGTP from profilactin. There is also minor dissociation in the buffer control, but much less compared to the DNaseI sample.

\subsection{Thermal stability analysis of Exportin 6 and its complexes}

Thermofluor assay detects the thermal denaturation of proteins via hydrophobic fluorescent dyes. The principles of the technique and the application areas are reviewed in (Boivin et al., 2013). The fluorescence signal from the dye is quenched in aqueous solutions, and it is enhanced when the dye binds to hydrophobic regions of proteins. For most globular proteins this occurs as the protein unfolds and the hydrophobic core is exposed. At low temperatures, measured fluorescence is low, which increases as the protein unfolds and it reaches its maximum during complete unfolding. As the temperature further increases, proteins start to aggregate, which decreases the accessibility of hydrophobic regions, hence the fluorescence signal drops. For NTRs, which have hydrophobic pockets on their surfaces, the situation is somewhat different. In our experiments, we used equal amounts $(5 \mu \mathrm{M})$ of free Xpo6, Xpo6•RanGTP, and the export complex in a thermofluor assay, where the temperature was increased step-wise from $30^{\circ} \mathrm{C}$ to $95^{\circ} \mathrm{C}$ by $1^{\circ} \mathrm{C}$ increments. We used 1x Sypro-Orange (Life Technologies) in the reaction as a fluorescent dye. Three replicates for each sample were measured. Figure 3-17 shows the fluorescence measurements of the Thermofluor assay. It is important to note that the fluorescence signal does not start from zero, as expected for globular proteins with a 
hydrophobic core and hydrophilic surface. This indicates that the fluorescent dye interacts with Xpo6 also in the folded state, most probably via the hydrophobic FG binding pockets. The starting level fluorescence signals for the export complex are even higher than those for the free Xpo6 and Xpo6•RanGTP. Nonetheless, a shifting trend for the fluorescence peaks of Xpo6, Xpo6 $\bullet$ RanGTP and the export complex can be seen. In order to determine the melting temperature (Tm), at which half of all molecules are denatured, the negative change in the fluorescence signal is calculated as a function of temperature (Figure 3-18). The Tm was calculated as $41^{\circ} \mathrm{C}, 44^{\circ} \mathrm{C}$ and $50^{\circ} \mathrm{C}$ for Xpo6, Xpo6 ${ }^{\circ}$ RanGTP and the export complex, respectively. These results clearly show that Xpo6 is stabilized by binding of RanGTP, and even further stabilized by binding of the cargo.

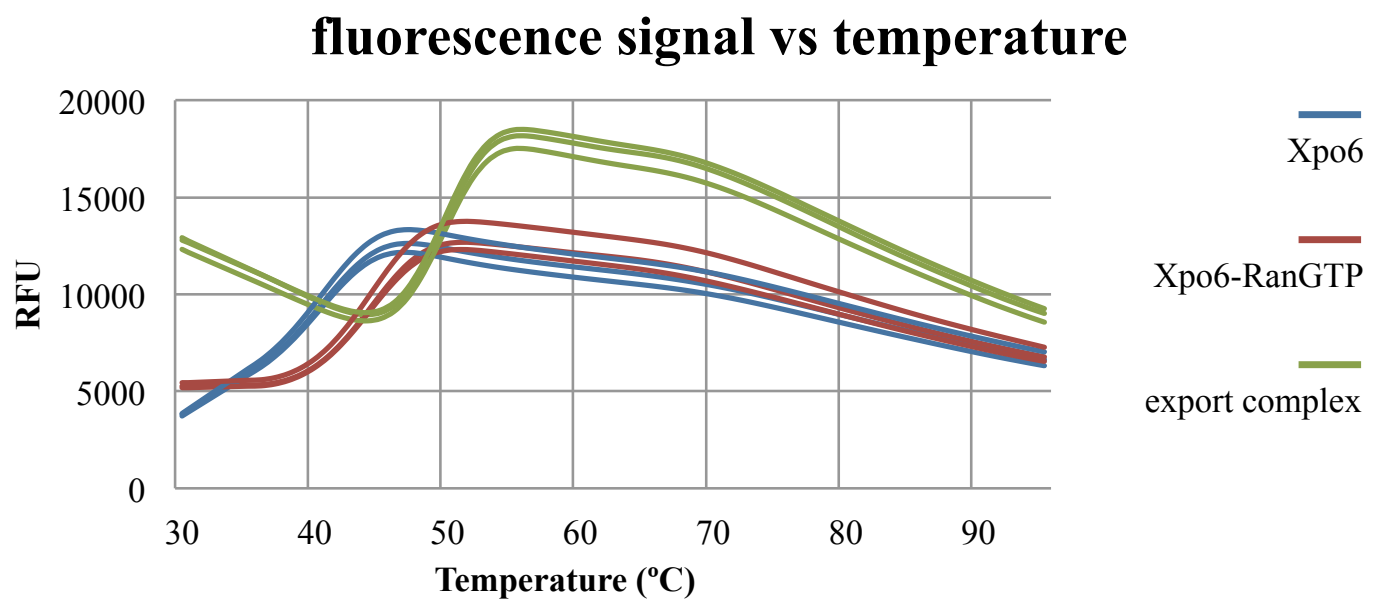

Figure 3-17 Themofluor measurements for Xpo6, Xpo6• RanGTP and the export complex

Sypro-Orange fluorescence signal in relative fluorescence units (RFU) versus temperature. Three lines of each color represent the technical repeats for the respective sample; blue: Xpo6, red: Xpo6•RanGTP, green: export complex. Note that the initial fluorescence signal is different than zero, and that this signal is far greater for the export complex than for $\mathrm{Xpo6} \cdot \mathrm{RanGTP}$ and the free Xpo6.

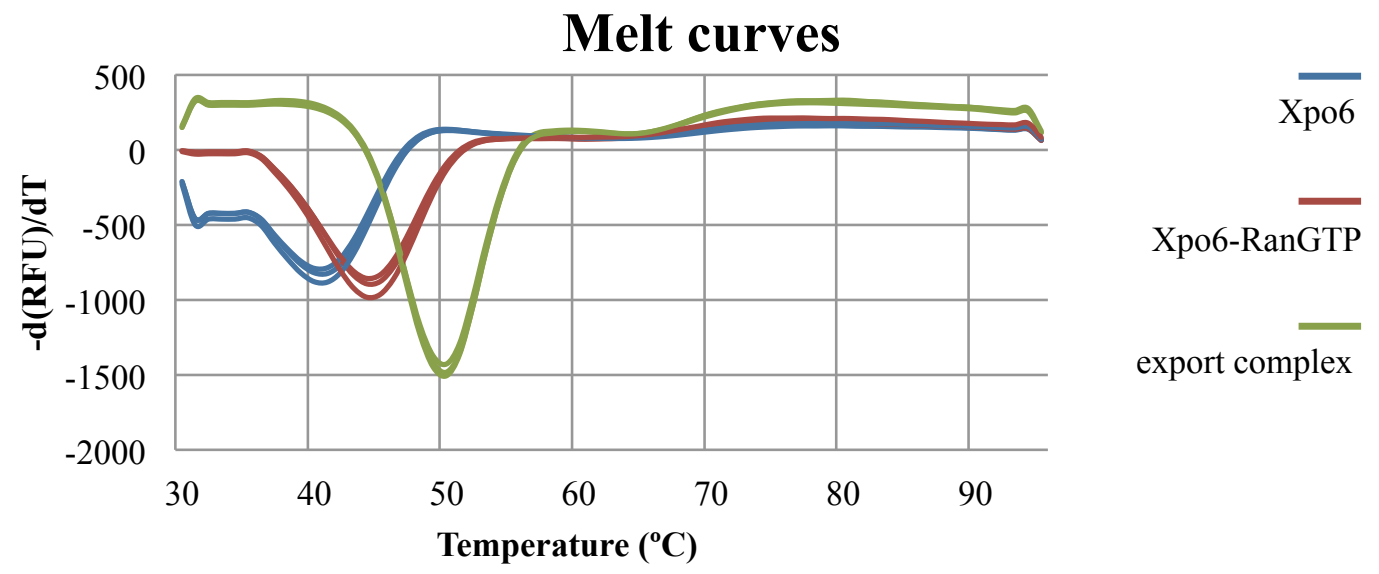

Figure 3-18 Melting curves for Xpo6, Xpo6• RanGTP and the export complex

The graph shows the derivative of the fluorescence signal versus temperature. Three lines of each color represent the technical repeats for the respective sample; blue: Xpo6, red: Xpo6•RanGTP, green: export complex. The dips indicate the melting temperatures: $41^{\circ} \mathrm{C}, 44^{\circ} \mathrm{C}, 50^{\circ} \mathrm{C}$ for free Xpo6, Xpo6 $\bullet$ RanGTP and the export complex, respectively. Melting temperature $(\mathrm{Tm})$ indicates the thermostability of proteins and complexes in the particular buffer condition. Note the increase in thermostability of Xpo6 with more binding partners bound to it. 


\subsection{Crystallization of Exportin 6 and its complexes}

\subsubsection{Crystallization trials with Xpo6•RanGTP complex}

We have used highly pure and concentrated Xpo6•RanGTP complex (Figure 3-6) for crystallization trials. An important quality for proper crystallization substrates is the homogeneity of the sample. We used dynamic light scattering (DLS) to assess the quality of our sample. The principles of the techniques are briefly described in 6.2.10. The purified complex concentrated to $15 \mathrm{mg} / \mathrm{mL}$ was exceptionally pure with polydispersity values of $5-8 \%$ (data not shown). As a rule of thumb, samples with polydispersity lower than $15 \%$ are considered to be good for crystallization (Geerlof et al., 2006). We used commercially available sparse-matrix screens and a robotic system to screen a large variety of crystallization conditions. We have tested two temperatures $\left(4^{\circ} \mathrm{C}\right.$ and $\left.20^{\circ} \mathrm{C}\right)$ for each condition, which brought the crystallization conditions we screened to an estimated 3000 . However, we couldn't identify promising hits for crystallization of this complex

\subsubsection{Crystallization of Exportin 6}

During the crystallization trials of the Xpo6•RanGTP complex, we found a condition with crystals, which we wanted to reproduce and improve. For the optimization screens, we used free Xpo6 as a control. The initial crystals were later found to be salt crystals and hence could not be reproduced. But in the optimization screens performed in the meantime, we saw that free Xpo6 showed crystalline like growth, unlike the Xpo6 $\bullet$ RanGTP complex. This showed us that the free Xpo6 had potential to crystallize on its own. Xpo6 was purified over gel filtration in a low salt buffer (10 Tris pH 7.5, $10 \mathrm{NaCl}, 1 \mathrm{DTT})$. During concentration, the purified protein started to precipitate, which could be reversed by the increase of salt to $50 \mathrm{mM}$. It was possible to concentrate the purified protein (in 10 Tris $\mathrm{pH}$ $7.5,50 \mathrm{NaCl}, 1 \mathrm{DTT})$ up to $25 \mathrm{mg} / \mathrm{mL}$. The sample homogeneity was good, indicated by $11 \%$ polydispersity in dynamic light scattering measurements (data not shown). Several protein concentrations $(8,17,25 \mathrm{mg} / \mathrm{mL})$ were tried for the initial trials. The first plates already showed that $8 \mathrm{mg} / \mathrm{mL}$ was too low (all drops remained clear) and $25 \mathrm{mg} / \mathrm{mL}$ was too high (heavy precipitation in all drops) for crystallization. $17 \mathrm{mg} / \mathrm{mL}$ protein concentration resulted in a dynamic precipitation behavior, which is desirable for crystallization. We used the robotic system for a high throughput screening of available commercial screens. Each screen was tested at two temperatures $\left(4^{\circ} \mathrm{C}-20^{\circ} \mathrm{C}\right)$, which multiplied the number of conditions. Several different conditions were identified in the 
first couple of days, where crystals or crystalline precipitations were observed. All of these conditions included polymer-based precipitants such as different Polyethylene glycol (PEG) polymers, Jeffamine or a variety of Sokalan ${ }^{\circledR}$ polymers. Jeffamine is a polyether diamine polymer, which is similar to and slightly more hydrophobic than PEG. Sokalan ${ }^{\circledR}$ is a brand name for a group of hydrophilic copolymers. Sokalan ${ }^{\circledR}$ CP5 and CP 7 are maleic acid/acrylic acid copolymers and Sokalan ${ }^{\circledR}$ CP $12 \mathrm{~S}$ is a modified polyacrylic acid. Table 3-1 summarizes the initial hits identified for Xpo6 crystallization. All hits were obtained at $4{ }^{\circ} \mathrm{C}$. Selected images from the crystal drops are shown in Figure 3-19.

Table 3-1 Initial crystallization conditions identified for Xpo6

\begin{tabular}{|c|c|c|c|c|c|c|c|c|}
\hline screen/condition & buffer & pH & precipitant & salt & alcohol & $\begin{array}{c}\text { Protein } \\
\text { concentration }\end{array}$ & scoring & $\begin{array}{l}\text { Time } \\
\text { scale }\end{array}$ \\
\hline MIDAS H10 & $\begin{array}{c}100 \mathrm{mM} \\
\text { Tris } \mathrm{HCl}\end{array}$ & 8.5 & $\begin{array}{c}25 \% \\
\text { Sokalan } \\
\text { CP5 }\end{array}$ & - & - & $17 \mathrm{mg} / \mathrm{mL}$ & $\begin{array}{l}\text { rod shaped } \\
\text { crystals } \\
\text { from clear } \\
\text { drops }\end{array}$ & day 1 \\
\hline MIDAS H9 & $\begin{array}{c}100 \mathrm{mM} \\
\mathrm{HEPES} \\
\mathrm{NaOH}\end{array}$ & 7 & $\begin{array}{c}25 \% \\
\text { Sokalan } \\
\text { HP56 }\end{array}$ & $\begin{array}{c}200 \mathrm{mM} \\
\text { Ammonium } \\
\text { acetate }\end{array}$ & - & $17 \mathrm{mg} / \mathrm{mL}$ & $\begin{array}{l}\text { crystals are } \\
\text { not } \\
\text { regularly } \\
\text { shaped }\end{array}$ & day 3 \\
\hline MIDAS G11 & $\begin{array}{l}100 \mathrm{mM} \\
\mathrm{Bis} \text { tris } \\
\mathrm{NaOH}\end{array}$ & 5.5 & $\begin{array}{c}15 \% \\
\text { Sokalan } \\
\text { CP12 S }\end{array}$ & $\begin{array}{l}\text { 100mM tri- } \\
\text { lithium } \\
\text { citrate } \\
\text { tetrahydr. }\end{array}$ & - & $17 \mathrm{mg} / \mathrm{mL}$ & $\begin{array}{l}\text { rod shaped } \\
\text { thick } \\
\text { crystals } \\
\text { (can be } \\
\text { hexagonal) }\end{array}$ & day3 \\
\hline MIDAS G1 & $\begin{array}{c}100 \mathrm{mM} \\
\mathrm{HEPES} \\
\mathrm{NaOH}\end{array}$ & 7 & $\begin{array}{c}25 \% \\
\text { Sokalan } \\
\text { CP7 }\end{array}$ & $\begin{array}{l}100 \mathrm{mM} \\
\text { Potassium } \\
\text { chloride }\end{array}$ & - & $17 \mathrm{mg} / \mathrm{mL}$ & $\begin{array}{l}\text { roundish } \\
\text { crystals } \\
\text { appear } \\
\text { from } \\
\text { precipitate }\end{array}$ & day 9 \\
\hline MIDAS F11 & $\begin{array}{c}100 \mathrm{mM} \\
\mathrm{HEPES} \\
\mathrm{NaOH}\end{array}$ & 7 & $\begin{array}{c}20 \% \\
\text { Sokalan } \\
\text { CP7 }\end{array}$ & $\begin{array}{l}100 \mathrm{mM} \\
\text { Ammonium } \\
\text { formate }\end{array}$ & - & $17 \mathrm{mg} / \mathrm{mL}$ & $\begin{array}{l}\text { roundish } \\
\text { crystals } \\
\text { appear } \\
\text { from } \\
\text { precipitate }\end{array}$ & day 9 \\
\hline MIDAS C1 & - & - & $\begin{array}{c}15 \% \\
\text { Jeffamine } \\
\text { ED2003 }\end{array}$ & - & $\begin{array}{c}10 \% \\
\text { EtOH }\end{array}$ & $17 \mathrm{mg} / \mathrm{mL}$ & $\begin{array}{l}\text { tiny rods } \\
\text { appear } \\
\text { from clear } \\
\text { drop }\end{array}$ & day 5 \\
\hline MBclass E12 & $\begin{array}{c}50 \mathrm{mM} \\
\text { Sodium } \\
\text { phosphate }\end{array}$ & 6.7 & $\begin{array}{c}5 \% \text { PEG } \\
4000\end{array}$ & - & - & $16.5 \mathrm{mg} / \mathrm{mL}$ & $\begin{array}{l}\text { irregular } \\
\text { rods }\end{array}$ & day 9 \\
\hline MBclass E9 & $\begin{array}{l}50 \mathrm{mM} \\
\text { Sodium } \\
\text { citrate }\end{array}$ & 5.6 & $\begin{array}{c}10 \% \text { PEG } \\
3350\end{array}$ & $\begin{array}{c}150 \mathrm{mM} \\
\mathrm{NaCl}\end{array}$ & - & $16.5 \mathrm{mg} / \mathrm{mL}$ & $\begin{array}{c}\text { rod shaped } \\
\text { crystals } \\
\text { (similar to } \\
\text { MidasH10) }\end{array}$ & day1 \\
\hline MBclass E8 & $\begin{array}{c}50 \mathrm{mM} \\
\text { Tris }\end{array}$ & 7.5 & $\begin{array}{l}12 \% \text { PEG } \\
\text { MME } 2000\end{array}$ & $\begin{array}{c}500 \mathrm{mM} \\
\mathrm{NaCl}\end{array}$ & - & $16.5 \mathrm{mg} / \mathrm{mL}$ & $\begin{array}{c}\text { oval } \\
\text { shaped } \\
\text { plates }\end{array}$ & $\begin{array}{l}\text { day } \\
1-3\end{array}$ \\
\hline
\end{tabular}



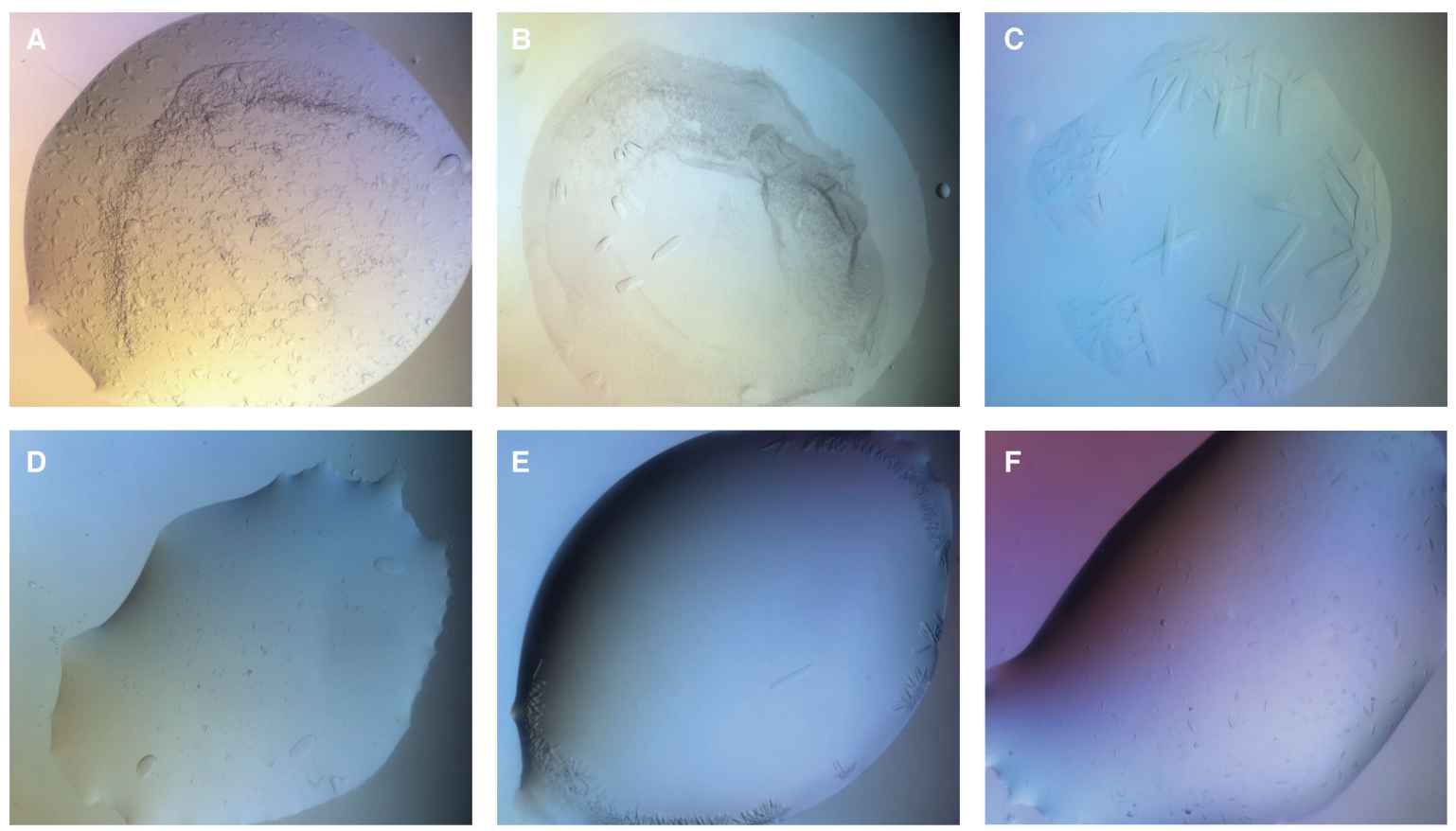

Figure 3-19 Initial crystallization hits for Xpo6

Crystals of Xpo6 grown in various crystallization conditions, all at $4^{\circ} \mathrm{C}$. A) MIDAS-G1: $100 \mathrm{mM}$ HEPES NaOH pH 7.0, $25 \%$ Sokalan ${ }^{\circledR}$ CP7, $100 \mathrm{mM} \mathrm{KCl} \mathrm{B)} \mathrm{MIDAS-G11:} 100 \mathrm{mM}$ Bis-Tris NaOH pH 5.5, 15\% Sokalan ${ }^{\circledR} \mathrm{CP} 12 \mathrm{~S}, 100 \mathrm{mM}$ lithium citrate C) MIDAS-H10: $100 \mathrm{mM}$ Tris $\mathrm{pH}$ 8.5, 25\% Sokalan ${ }^{\circledR}$ CP5 D) MBclass-E8: $50 \mathrm{mM}$ Tris pH 7.5, 12\% PEG MME 2000, $500 \mathrm{mM} \mathrm{NaCl}$ E) MBclass E9: $50 \mathrm{mM}$ sodium citrate $\mathrm{pH}$ 5.6, $10 \%$ PEG 3350, $150 \mathrm{mM} \mathrm{NaCl} \mathrm{F)} \mathrm{MBclass-}$ E12: $50 \mathrm{mM}$ sodium phosphate $\mathrm{pH}$ 6.7, 5\% PEG 4000.

The initial conditions we identified provided the base for the further crystallization screens. Refinement screens were prepared in a way that the buffer, salt, precipitant concentrations and the $\mathrm{pH}$ of the initial hits were varied in small increments to find better crystallization conditions. Table 3-2 summarizes the refinement screens we prepared, based on the initial crystallization hits for Xpo6. Some screens resulted in improved crystals, whereas some hits could not be reproduced. Also for the refinement conditions all crystals were obtained at $4^{\circ} \mathrm{C}$; no crystals appeared if the same conditions were incubated at $20^{\circ} \mathrm{C}$.

Especially the refinements based on initial hit "MBclass E8" yielded improved and reproducible crystals. In the initial drop there were oval-shaped two-dimensional plates, whereas the refinement screens resulted in hexagonal rod-shaped crystals. The crystallization window of Xpo6 in this particular condition was very broad. We obtained crystals at PEG MME 2000 concentrations between 5-9\%, at $\mathrm{NaCl}$ concentrations between 50-500 $\mathrm{mM}$ and in a $\mathrm{pH}$ range of 7.0-7.8. However, the appearance and the quality of the crystals changed within these limits. With increasing PEG MME 2000, nucleation was increased; the crystals were more in number, smaller and roundish. With increasing $\mathrm{NaCl}$, fewer and bigger crystals grew, but they were generally less sharp on their edges. The crystals were on average $60 \mu \mathrm{m}$ in length, but some of them grew as large as $300 \mu \mathrm{m}$. The 
crystals appeared within $24 \mathrm{~h}$ after the setting of the drop, and continue growing for a couple of days. Figure 3-20 shows some examples of Xpo6 crystals obtained from the refinement screens based on "MBclass E8".

Table 3-2 Refinement screen conditions for Xpo6 crystallization

\begin{tabular}{|c|c|c|c|c|c|c|}
\hline Initial hit & Initial condition & screened & precipitant & salt & pH & Crystal range \\
\hline MIDAS/G11 & $\begin{array}{c}\text { 100mM Bis tris } \mathrm{NaOH} \text { pH } \\
5.5 \\
15 \% \text { Sokalan CP } 12 \mathrm{~S} \\
100 \mathrm{mM} \text { lithium citrate }\end{array}$ & $\begin{array}{c}\mathrm{pH} \\
\mathrm{vs} \\
\text { precipitant }\end{array}$ & $10-20 \%$ & $100 \mathrm{mM}$ & $5.5-6.5$ & No crystals \\
\hline MIDAS G1 & $\begin{array}{c}\text { 100mM HEPES NaOH } \mathrm{pH} \\
7.0 \\
100 \mathrm{mM} \mathrm{KCl} \\
25 \% \text { Sokalan CP7 }\end{array}$ & $\begin{array}{c}\mathrm{pH} \\
\mathrm{vs} \\
\text { precipitant }\end{array}$ & $15-25 \%$ & $100 \mathrm{mM}$ & $6.5-7.5$ & $\begin{array}{c}\text { Rods } \\
\text { pH } 6.83 \\
\text { precipitant 21- } \\
25 \%\end{array}$ \\
\hline MIDAS F11 & $\begin{array}{c}\text { 100mM HEPES NaOH pH } \\
7.0 \\
100 \mathrm{mM} \text { ammonium } \\
\text { formate } \\
20 \% \text { Sokalan CP7 }\end{array}$ & $\begin{array}{c}\mathrm{pH} \\
\text { vs } \\
\text { precipitant }\end{array}$ & $15-25 \%$ & $100 \mathrm{mM}$ & $6.5-7.5$ & No crystals \\
\hline MBclass E12 & $\begin{array}{c}\text { 50mM Sodium phosphate } \\
\text { pH } 6.7 \\
\text { 5\% PEG } 4000 \\
\end{array}$ & $\begin{array}{c}\mathrm{pH} \\
\mathrm{vs} \\
\text { precipitant }\end{array}$ & $2.5-15 \%$ & - & $6.0-7.0$ & No crystals \\
\hline MBclass E12 & $\begin{array}{c}\text { 50mM Sodium phosphate } \\
\text { pH } 6.7 \\
5 \% \text { PEG } 4000\end{array}$ & $\begin{array}{c}\mathrm{pH} \\
\text { vs } \\
\text { precipitant }\end{array}$ & $4-6.5 \%$ & - & $5.1-6.8$ & $\begin{array}{c}\text { Rods } \\
\text { pH } 6.56 \\
\text { precipitant } 4 \%\end{array}$ \\
\hline MBclass E8 & $\begin{array}{c}\text { 50mM Tris } \mathrm{pH} 7.5 \\
500 \mathrm{mM} \mathrm{NaCl} \\
12 \% \text { PEG MME } 2000\end{array}$ & $\begin{array}{c}\mathrm{pH} \\
\mathrm{vs} \\
\text { precipitant }\end{array}$ & $5-20 \%$ & $500 \mathrm{mM}$ & $7.0-8.5$ & $\begin{array}{c}\text { Rods } \\
\text { pH 7.0-7.8 } \\
\text { precipitant 5- } \\
7.5 \%\end{array}$ \\
\hline MBclass E8 & $\begin{array}{c}\text { 50mM Tris } \mathrm{pH} 7.5 \\
500 \mathrm{mM} \mathrm{NaCl} \\
12 \% \text { PEG MME } 2000\end{array}$ & $\begin{array}{c}\text { salt } \\
\text { vs } \\
\text { precipitant }\end{array}$ & $5-20 \%$ & $\begin{array}{c}100-500 \\
\mathrm{mM}\end{array}$ & 7.5 & $\begin{array}{c}\text { Rods } \\
\text { salt } 100- \\
443 \mathrm{mM} \\
\text { precipitant 5- } \\
9 \%\end{array}$ \\
\hline MBclass E8 & $\begin{array}{c}\text { 50mM Tris pH } 7.5 \\
500 \mathrm{mM} \mathrm{NaCl} \\
12 \% \text { PEG MME } 2000\end{array}$ & $\begin{array}{l}\text { salt } \\
\text { vs } \\
\text { pH }\end{array}$ & $5 \%$ & $\begin{array}{c}50-500 \\
\mathrm{mM}\end{array}$ & $7.0-7.8$ & $\begin{array}{c}\text { Rods } \\
\text { salt } 50- \\
400 \mathrm{mM} \\
\text { pH } 7.0-7.5\end{array}$ \\
\hline MBclass E9 & $\begin{array}{c}\text { 50mM Sodium citrate } \mathrm{pH} \\
5.6 \\
150 \mathrm{mM} \mathrm{NaCl} \\
10 \% \text { PEG } 3350\end{array}$ & $\begin{array}{c}\mathrm{pH} \\
\mathrm{vs} \\
\text { precipitant }\end{array}$ & $5-15 \%$ & $150 \mathrm{mM}$ & $5.0-6.0$ & No crystals \\
\hline MBclass E9 & $\begin{array}{c}\text { 50mM Sodium citrate } \mathrm{pH} \\
5.6 \\
150 \mathrm{mM} \mathrm{NaCl} \\
10 \% \text { PEG } 3350\end{array}$ & $\begin{array}{l}\text { salt } \\
\text { vs } \\
\text { precipitant }\end{array}$ & $5-15 \%$ & $0-300 \mathrm{mM}$ & 5.6 & No crystals \\
\hline
\end{tabular}



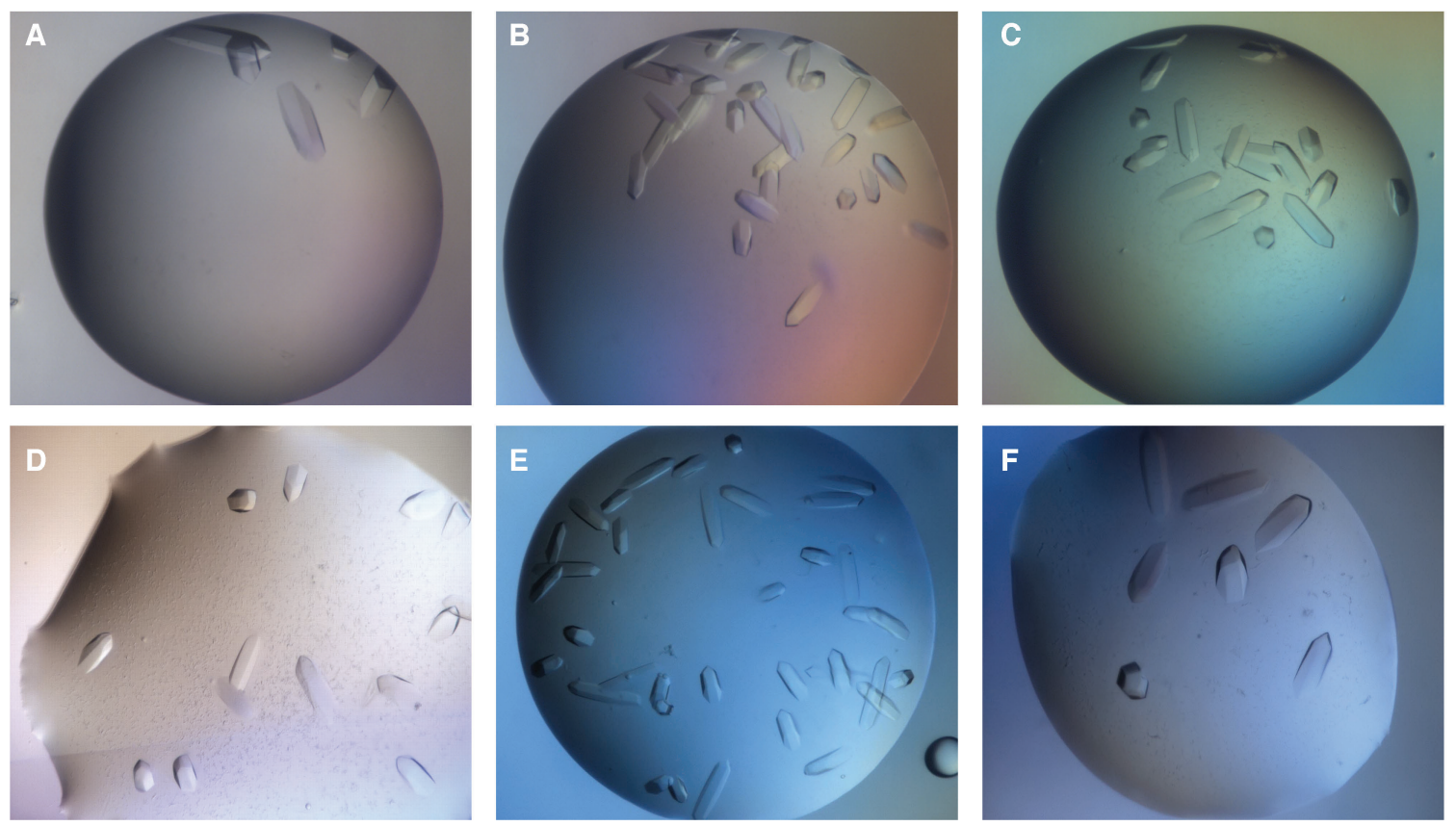

Figure 3-20 Xpo6 crystals from the refinement of "MBclass E8"

Crystals of Xpo6 grown in the refinement screens based on MBclass-E8 condition, all at $4^{\circ} \mathrm{C}$. A) $50 \mathrm{mM}$ Tris pH 7.6, 100 $\mathrm{mM} \mathrm{NaCl}$, 5.09\% PEG MME 2000, B) $50 \mathrm{mM}$ Tris pH 7.39, $100 \mathrm{mM} \mathrm{NaCl}, 5.73 \%$ PEG MME 2000, C) $50 \mathrm{mM}$ Tris $\mathrm{pH}$ 7.6, $100 \mathrm{mM} \mathrm{NaCl}, 5.73 \%$ PEG MME 2000, D) $50 \mathrm{mM}$ Tris pH 7.0, $500 \mathrm{mM} \mathrm{NaCl}, 6.36 \%$ PEG MME 2000, E) $50 \mathrm{mM}$ Tris pH 7.64, $500 \mathrm{mM} \mathrm{NaCl}, 5 \%$ PEG MME 2000, F) $50 \mathrm{mM}$ Tris pH 7.5, $368 \mathrm{mM} \mathrm{NaCl,} \mathrm{5 \%} \mathrm{PEG} \mathrm{MME} 2000$

The refinement screens for "MBClass E12" hit revealed only one single condition where hexagonal rod shaped crystals appeared; at $50 \mathrm{mM}$ sodium phosphate $\mathrm{pH} 6.56$ with $4 \%$ PEG 4000. The crystallization window for this condition was so narrow, that even small changes in $\mathrm{pH}$ were not tolerated. The crystals appeared on day 1, and continue growing until day 5. We harvested this drop on day 5. At pH 6.56, PEG concentrations higher than $4 \%$ produced crystals that were smaller and roundish. These drops became too crowded with many small crystals, such that harvesting was not possible. The refinement screen based on "Midas G1" yielded rod-shaped crystals at 100 mM HEPES pH 6.83 and 100mM $\mathrm{KCl}$, which got bigger with the increasing precipitant concentration (21-25\% Sokalan $^{\circledR}$ CP7). Figure 3-21 shows improved crystals from the refinement screens based on "MBClass E12" and "Midas G1".

For the X-ray diffraction analysis the crystals were frozen and transported to the synchrotron. Prior to freezing, crystals are brought into a cryo-condition, which causes amorphous freezing of the solution in liquid nitrogen. Crystalline ice is undesired as it interferes with the diffraction analysis of the protein crystals. Glycerol, ethylene glycol, and different sugars or alcohols can be used as cryoprotectants. 

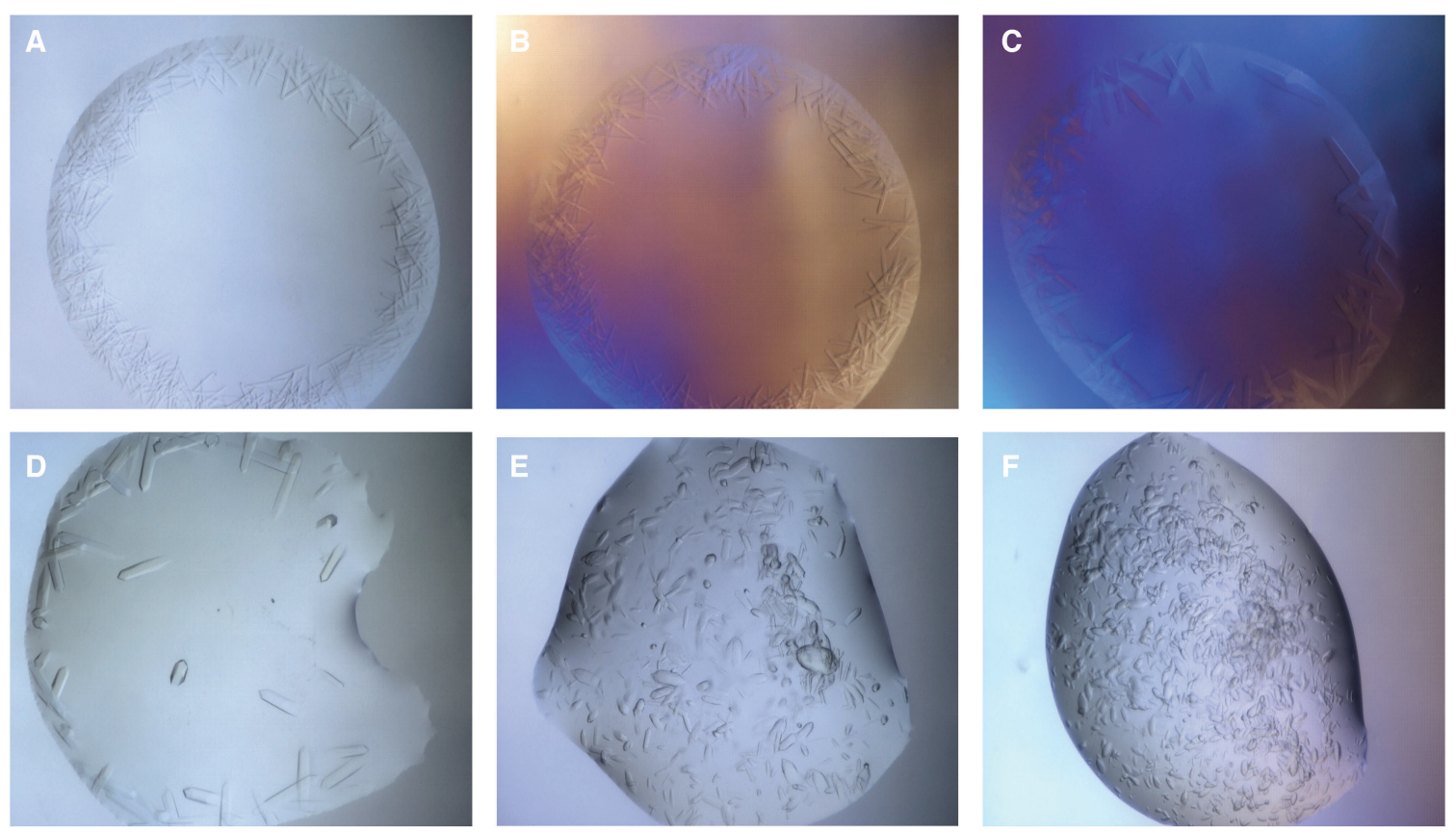

Figure 3-21 Xpo6 crystals from refinement of "Midas G1" and "MBclassE12"

A-C: Crystals of Xpo6 grown in the refinement screens based on MIDAS G1 condition, all at $4^{\circ} \mathrm{C}$. A) $100 \mathrm{mM}$ HEPES $\mathrm{NaOH} \mathrm{pH}$ 6.83, $100 \mathrm{mM} \mathrm{KCl}, 22.3 \%$ Sokalan $^{\circledR}$ CP7, B) $100 \mathrm{mM}$ HEPES NaOH pH 6.83, $100 \mathrm{mM} \mathrm{KCl,} 23.2 \%$ Sokalan ${ }^{\circledR}$ CP7, C) $100 \mathrm{mM}$ HEPES NaOH pH 6.83, $100 \mathrm{mM} \mathrm{KCl}, 24.1 \%$ Sokalan ${ }^{\circledR}$ CP7. D-F: Crystals of Xpo6 grown in the refinement screens based on MBclass E12 condition, all at $4^{\circ} \mathrm{C}$ D) $50 \mathrm{mM}$ sodium phosphate $\mathrm{pH} 6.56,4 \%$ PEG 4000, E) $50 \mathrm{mM}$ sodium phosphate $\mathrm{pH} 6.56,4,23 \%$ PEG 4000, F) $50 \mathrm{mM}$ sodium phosphate $\mathrm{pH} 6.56,4 \%$ PEG 4000

Cryo-conditions were prepared for each condition separately. Buffer and salt concentrations and $\mathrm{pH}$ were kept the same as the crystallization condition, the precipitant concentration was increased to $25 \%$ and $15 \%$ glycerol was added. The crystals were slowly acclimatized to the cryo-condition in order to minimize damage. We could not identify an appropriate cryo-solution for Sokalan ${ }^{\circledR}$ CP7 conditions, as the solution crystallized in liquid nitrogen, despite the addition of glycerol up to $25 \%$. The crystals from the refinement screen based on Sokalan ${ }^{\circledR}$ CP7 could therefore not be analyzed for their diffraction. We analyzed the diffractions of other crystals at Swiss Light Source PX II beam line. The best resolution was obtained from Xpo6 crystals grown in refinement screens "MBclass E8", and the crystals diffracted to $7.4 \AA$. We could determine the space group $\left(152, \mathrm{P} 3{ }_{1} 21\right)$ and unit cell dimensions $\left(203,203,258 \AA / 90^{\circ}, 90^{\circ}, 120^{\circ}\right)$. The crystals grown in different conditions were found to belong to the same space group with similar unit cell dimensions (Figure 3-22). The crystals grown in PEG MME 2000 conditions (MBclass E8 refinements) were the most reproducible crystals, with the best diffractions obtained $(7.4 \AA)$. However, we could not detect a trend between the diffraction quality and the salt, precipitant concentrations and $\mathrm{pH}$ of the conditions. 
A) PEG MME 2000 crystals - space group P3,21 trigonal, resolution $7.4 \AA$
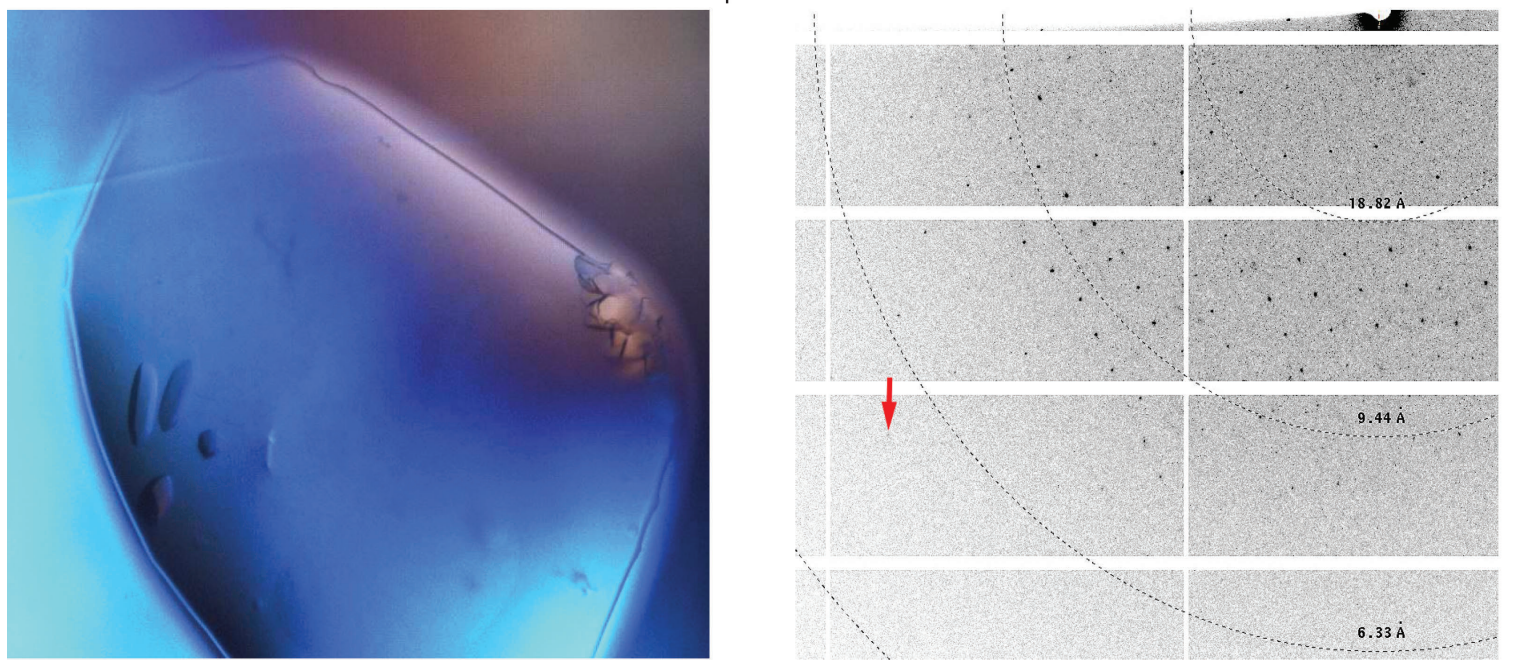

B) PEG 4000 crystals- no diffraction detected
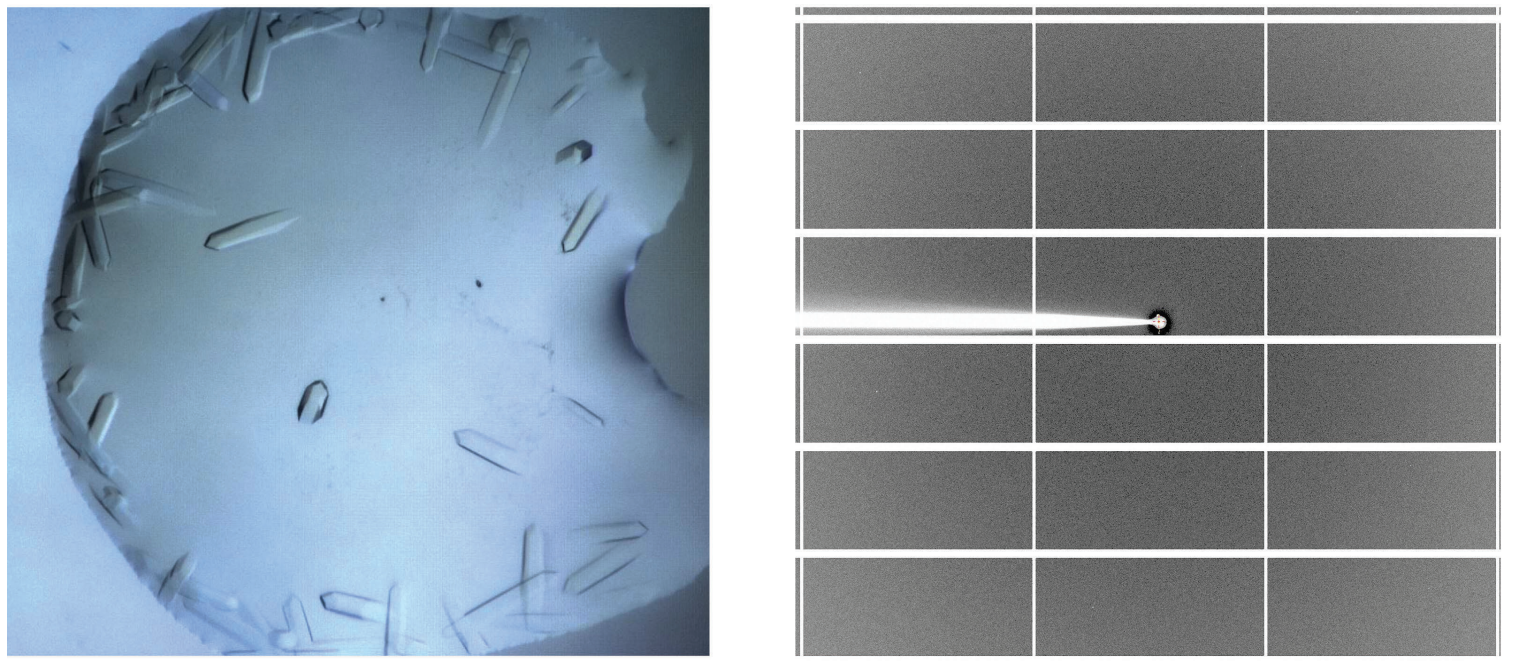

C) Sokalan® CP12S crystals - trigonal P3,21 - resolution $9 \AA$
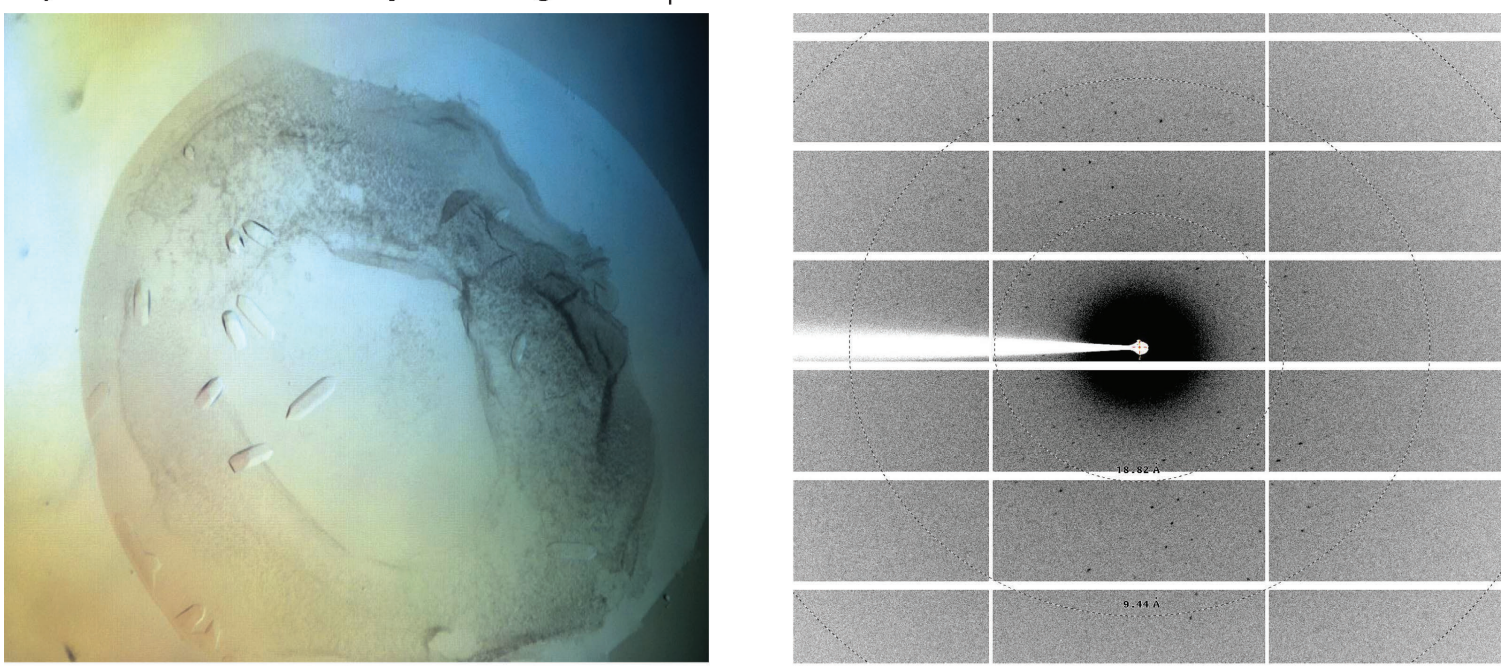

Figure 3-22 Xpo6 crystals and their diffractions

Representative images of Xpo6 crystals and their diffraction patterns. The diffraction data were collected at the Swiss Light source, PXII beamline, at $1 \AA \AA$ wavelength. So far the best diffraction were obtained from the crystals shown in A. Space group of the crystals in $\mathrm{A}$ and $\mathrm{C}$ were found to be the same, although grown in different conditions. No diffraction could be detected for the crystals shown in B. 


\subsubsection{Limited proteolysis and secondary structure prediction of Xpo6}

Flexible regions in proteins or protein complexes may interfere with proper crystal packing, and limit diffraction resolution of crystals. Such flexible regions can be identified by limited proteolysis. Proteases first target the most accessible regions such as loops or flexible ends rather than the folded core of the protein. When proteases are used in very low amounts, the proteolysis pattern can be used to identify the flexible regions of a protein. These regions may then be removed in order to obtain crystallizable products or to improve diffraction of the crystals. For Xpo6, we used three different proteases in limited proteolysis assay. Trypsin cleaves C-terminally at basic residues such as lysine and arginine. Chymotrypsin cleaves C-terminally at aromatic residues such as tryptophan, tyrosine and phenylalanine but also of other hydrophobic residues such as methionine and isoleucine. Glu-C cleaves C-terminally at the acidic glutamate residues. A serial dilution of the proteases in low concentrations was used to obtain a digestion pattern (for concentrations and the protocol see 6.2.11). The digestion pattern is shown in Figure 3-23. The indicated bands were analyzed by mass spectrometry to identify the protease cleavage sites. The identified Trypsin and chymotrypsin digestion sites were towards the C-terminus of the protein whereas Glu-C digested at one site towards the N-terminus of the protein.

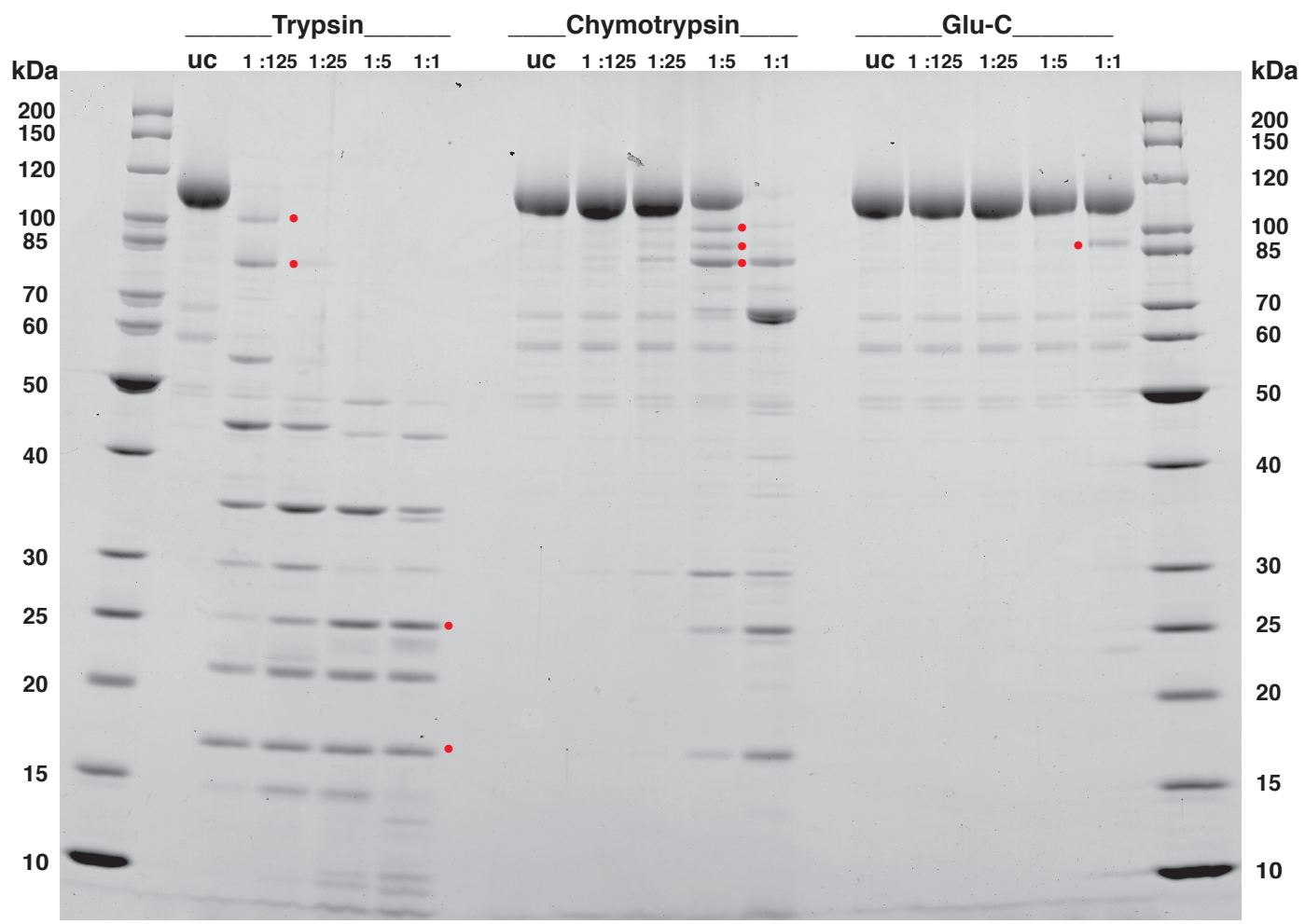

Figure 3-23 Limited proteolysis of Xpo6

$3 \mu \mathrm{g}$ of Xpo6 was incubated with varying concentrations of different proteases for $1 \mathrm{~h}$ at $22{ }^{\circ} \mathrm{C}$. The digestion pattern was analyzed by SDS-PAGE and the fragments indicated with red stars were analyzed by mass spectrometry. The fraction numbers indicate the dilution factor of the protease used for the reaction from the working stock: $0.1 \mathrm{mg} / \mathrm{mL}$. UC: uncut sample with no protease. 

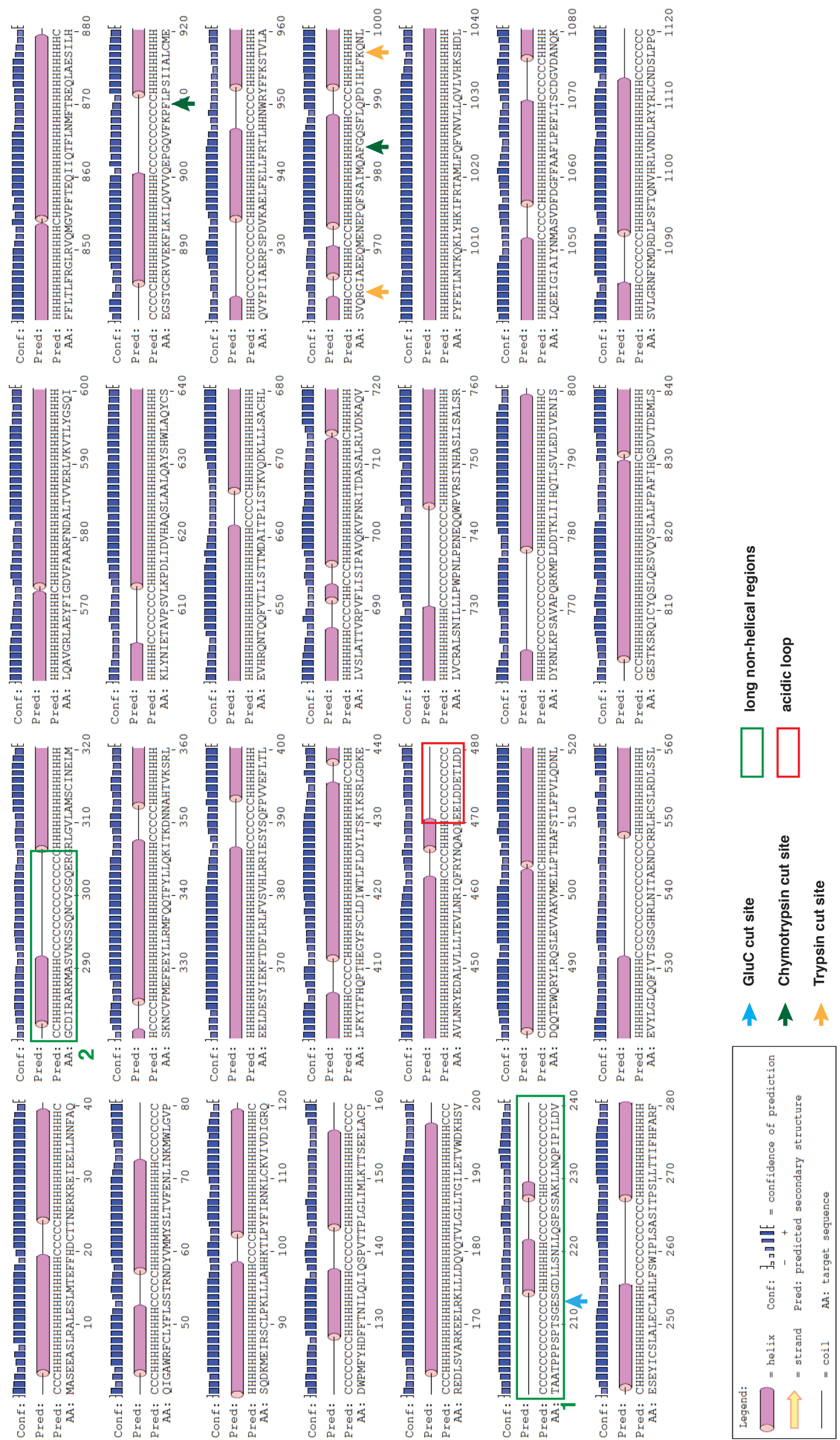

Figure 3-24 Secondary structure prediction for Xpo6 with "psipred" and identified protease cleavage sites 
In parallel, we ran secondary structure predictions with Xpo6 to compare with the limited proteolysis results. We used online secondary structure prediction tools such as Psipred and Consensus (see 6.1.7). Figure 3-24 shows a secondary structure prediction from Psipred online tool. The identified protease cleavage sites are also indicated in the figure.

Two long stretches without helix predictions was the first striking outcome of the structure predictions. The two regions were identified as 1 (aa 200-240) and 2 (aa 280-300) in the Figure 3-24. The helices predicted within these regions were shorter than the typical HEAT repeat helices, and not every prediction algorithm could predict helices in these regions. A very encouraging finding was that the identified Glu-C cut-site was within the first loop region. Trypsin and chymotrypsin cut-sites corresponded to small inter- or intrahelix loops towards the C-terminus. We also found a very acidic stretch corresponding to a helix-free region. By analogy with other nuclear export receptors, we infer that this region (aa 470-480) might correspond to the "acidic loop" that serves as an important binding site for RanGTP. We created several truncations of these three regions, in order to obtain a less-flexible crystallization substrate. We first compared the gel filtration profiles of the truncations with the full length Xpo6. The truncations of the second loop and the acidic loop eluted at the same volume as the full length Xpo6, whereas all truncations of the first loop eluted $0.2 \mathrm{~mL}$ later in a SD200 10/30 column (Figure 3-25). This indicates that the removal of the first loop made Xpo6 smaller in overall size. This can be possible if the region is located on the outer surface of Xpo6. Next, we analyzed the truncated Xpo6 versions for their ability to form complexes with RanGTP and profilactin. The binding assay was performed as described in 3.5.1. Table 3-3 summarizes the Xpo6 truncation constructs and their properties such as gel filtration behavior, aggregation and the ability to form an export complex. 


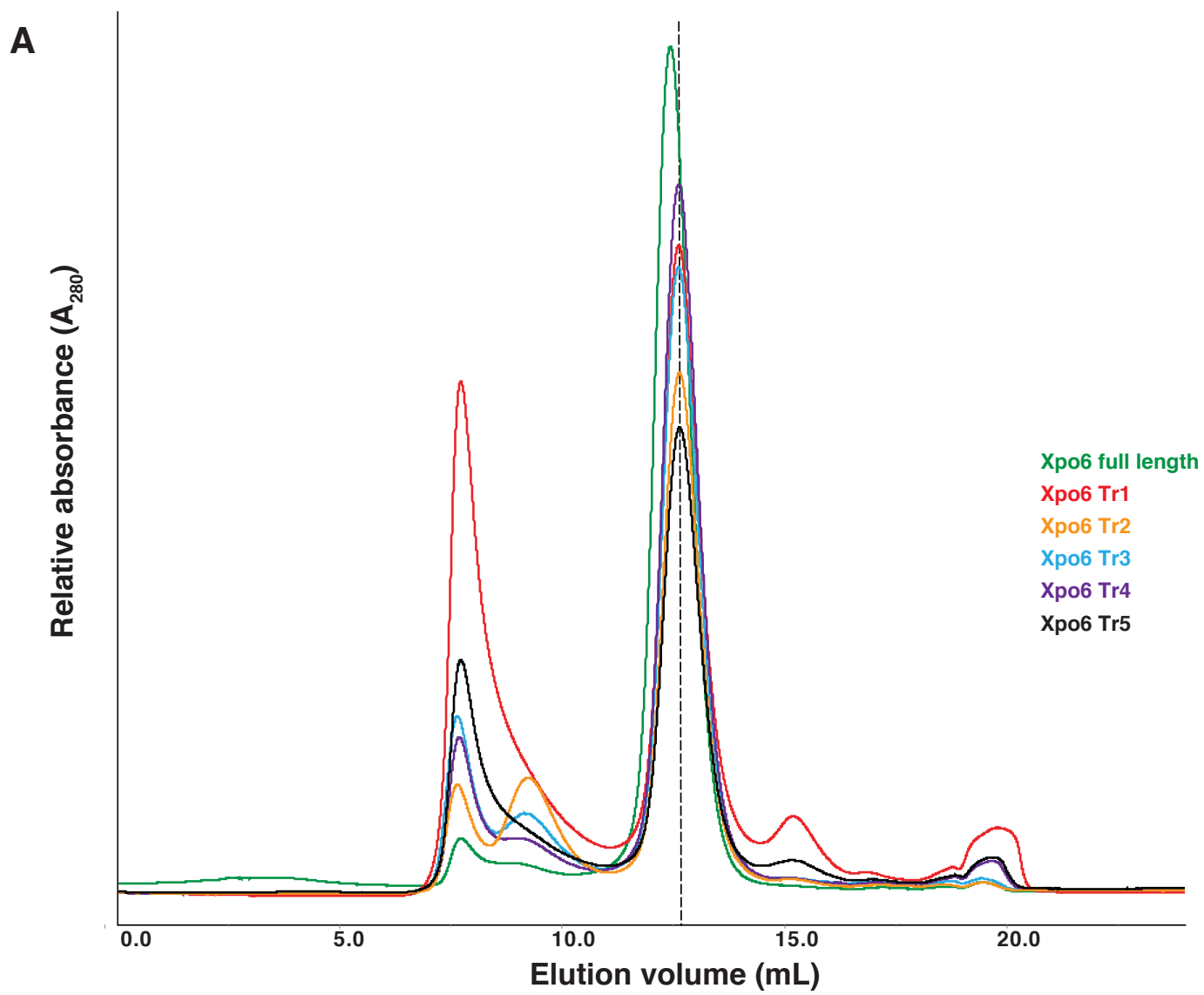

B

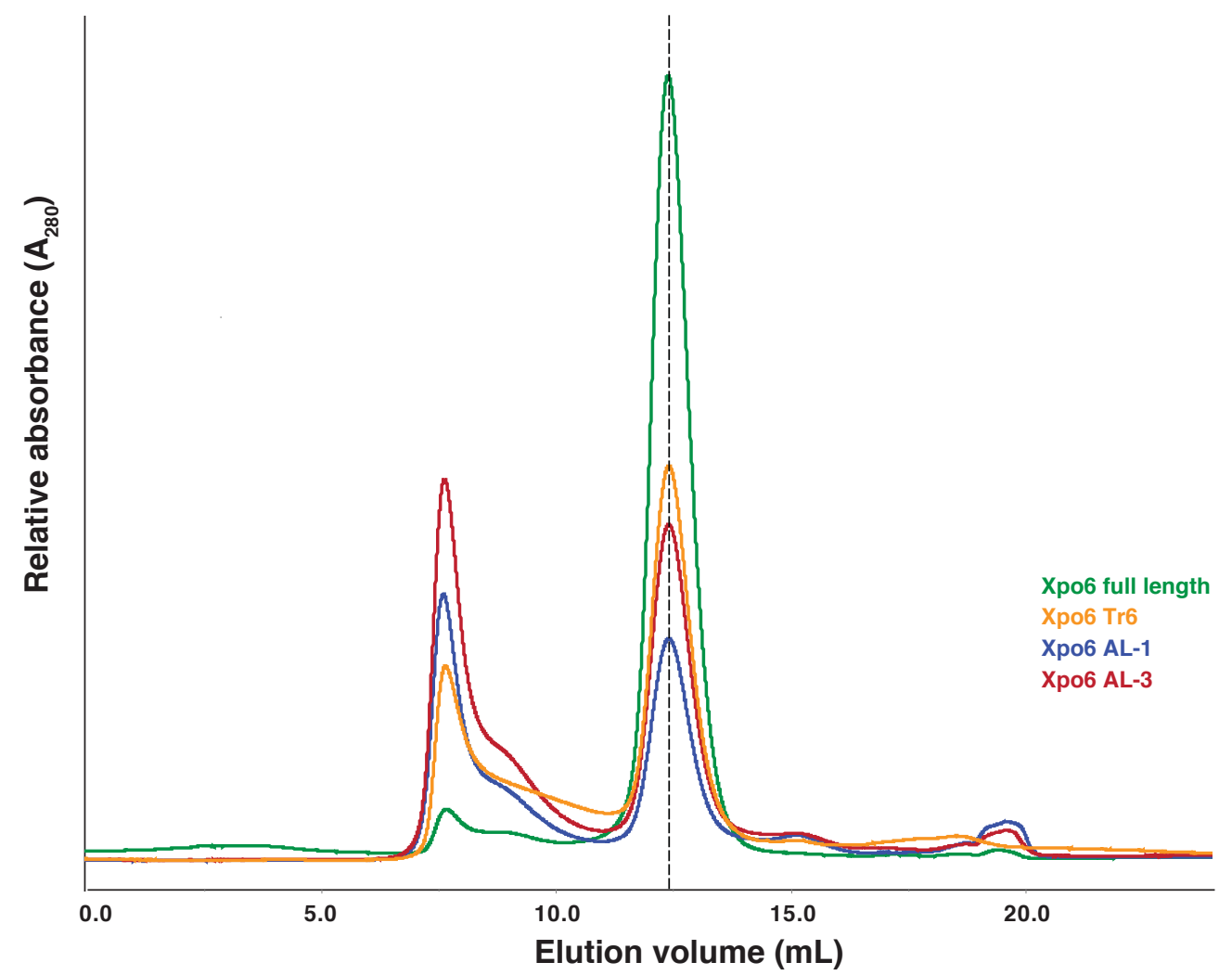

Figure 3-25 Gel filtration profiles of Xpo6 truncations

Gel filtration profiles of the full length and truncated human Xpo6 versions in $50 \mathrm{mM}$ Tris pH 7.5, $500 \mathrm{mM} \mathrm{NaCl}, 5 \mathrm{mM}$ DTT. The chromatograms are overlaid to scale. A) Gel filtration chromatograms of the truncations of the first loop versus full length Xpo6. Note that there is a shift of the peak by $0.2 \mathrm{~mL}$, constant for all truncations. B) Gel filtration 
chromatograms of the truncations of the second and acidic loops versus full length Xpo6. Note that the Xpo6 versions elute at the exact same volume.

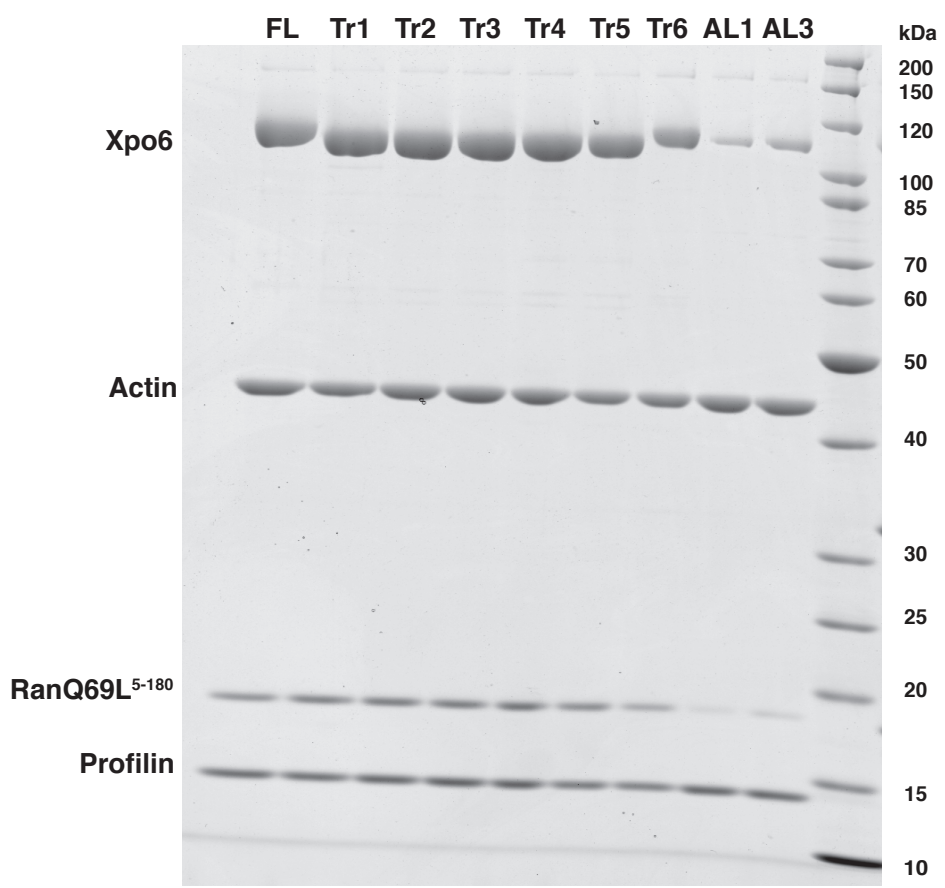

Figure 3-26 Actin export complex formation with Xpo6 truncations

Full length Xpo6 and the truncated versions are compared for their ability to form an export complex. The experimental setup was identical to Figure 3-10. The buffer conditions for binding were kept identical: $10 \mathrm{mM}$ Tris pH 7.5, $20 \mathrm{mM}$ $\mathrm{NaAc}, 1 \mathrm{mM}$ DTT, whereas each reaction had a different Xpo6 variant. Complex was immobilized through profilin, and the amount of Xpo6•RanGTP bound to profilactin was used to judge the complex stability.

Table 3-3 Xpo6 loop truncations and their properties

(\% Aggregation was calculated by the area of the void volume divided by the sum of the void and peak areas)

\begin{tabular}{|c|c|c|c|c|c|}
\hline Name & $\begin{array}{l}\text { Deletion } \\
\text { region }\end{array}$ & Deleted sequence & $\begin{array}{l}\text { Retention } \\
\text { time in } \\
\text { GF }(\mathrm{mL})\end{array}$ & $\begin{array}{l}\text { Aggregation } \\
\%\end{array}$ & $\begin{array}{l}\text { Complex } \\
\text { formation }\end{array}$ \\
\hline $\begin{array}{l}\text { Full } \\
\text { length }\end{array}$ & - & ( & 12.44 & $5 \%$ & yes \\
\hline Tr1 & Loop 1 & $\begin{array}{l}{ }^{201}{ }^{T A A T P P P S P T S G E S G D L L S N L L Q S P S S A ~} \\
\text { KLLNQPIPILDV }\end{array}$ & 12.62 & $46 \%$ & yes \\
\hline Tr2 & Loop 1 & $\begin{array}{l}{ }^{201} \text { TAATPPPSPTSGESGDLLSNLLQSPSSA } \\
\text { KLLNQPIPIL }{ }^{238}\end{array}$ & 12.64 & $29 \%$ & yes \\
\hline Tr3 & Loop 1 & $\begin{array}{l}{ }^{202} \text { AATPPPSPTSGESGDLLSNLLQSPSSAK } \\
\text { LLNQPIPIL }{ }^{238}\end{array}$ & 12.63 & $26 \%$ & yes \\
\hline Tr4 & Loop 1 & $\begin{array}{l}{ }^{203} \text { ATPPPSPTSGESGDLLSNLLQSPSSAKL } \\
\text { LNQPIPIL }{ }^{238}\end{array}$ & 12.63 & $19 \%$ & yes \\
\hline Tr5 & Loop 1 & $\begin{array}{l}{ }^{205} \text { PPPSPTSGESGDLLSNLLQSPSSAKLLN } \\
\text { QPIPILDV }{ }^{240}\end{array}$ & 12.64 & $36 \%$ & yes \\
\hline Tr6 & Loop 2 & ${ }^{281}$ GCDIRARKMASVNGSSQNCVSGQER ${ }^{305}$ & 12.45 & $38 \%$ & yes \\
\hline AL-1 & $\begin{array}{l}\text { Acidic } \\
\text { loop }\end{array}$ & ${ }^{469}$ QLEELDDETLDDDQQ ${ }^{483}$ & 12.44 & $56 \%$ & no \\
\hline AL-3 & $\begin{array}{l}\text { Acidic } \\
\text { loop }\end{array}$ & ${ }^{471}$ EELDDETLDDDQQ ${ }^{483}$ & 12.45 & $55 \%$ & no \\
\hline
\end{tabular}


As seen in Figure 3-26, Xpo6 versions with truncations in the first loop (Tr1-5) and truncation in the second loop (Tr6) retain their ability to form an export complex. This outcome further implied that the removal of these predicted loops did not disturb the overall folding and function of Xpo6. On the other hand, truncation of the acidic loop (AL1, AL3) severely hindered complex formation, indicating that this region might be involved in direct interactions with RanGTP and/or the cargo. We have chosen 2 truncation constructs of the first loop ( $\operatorname{Tr} 3$ and $\operatorname{Tr} 4)$ and one truncation construct of the second loop (Tr6) and used them in crystallization trials. With the truncated versions, we have screened the refined conditions for Xpo6 crystallization, as well as a wide range of commercial sparse-matrix screens, but we couldn't identify suitable conditions for crystallization so far.

\subsubsection{Sequence conservation of Xpo6 in evolutionary distant species}

In parallel to the secondary structure predictions, we also analyzed the sequence conservation of Xpo6 in other species. We used the position-specific-iterated BLAST (PSI-BLAST) tool of NCBI, which was more powerful than normal BLAST tools in identifying the Xpo6 sequences with low overall conservation. We specifically looked at the conservation of the predicted loop regions. Loops that are not directly involved in cargo recognition are more likely to be divergent. Indeed, we found that the conservation in these regions were lower than the regions that corresponded to predicted helices. Especially in evolutionary distant species like the tunicate Ciona intestinalis and the slime mold Dictyostelium species, these loop regions were significantly shorter. Also in the Xpo6 sequences from Xenopus laevis and Xenopus tropicalis, the loop regions are strikingly less conserved compared to the rest of the protein. Also second loop region has an insertion in all Xenopus isoforms we analyzed. This was a further indication that the deletion regions corresponded to loops that are not essential for Xpo6 function. Xpo6 from birds and mammals showed quite high conservation throughout the entire sequence. Figure 3-27 shows the Xpo6 alignments from selected species in comparison to human Xpo6.

Our loop truncations on Xpo6 might be very crude and have negative effect on the overall fold of the protein, and may hinder crystallization, rather than improving. Using an Xpo6 version that is trimmed over millions of years by evolution might be a more elegant approach to solve the problem with flexibility. We have chosen Xpo6 of two species, Dictyostelium purpureum and Dictyostelium fasciculatum for later crystallization studies. 
A) Sequence alignment of human and Xenopus Xpo6

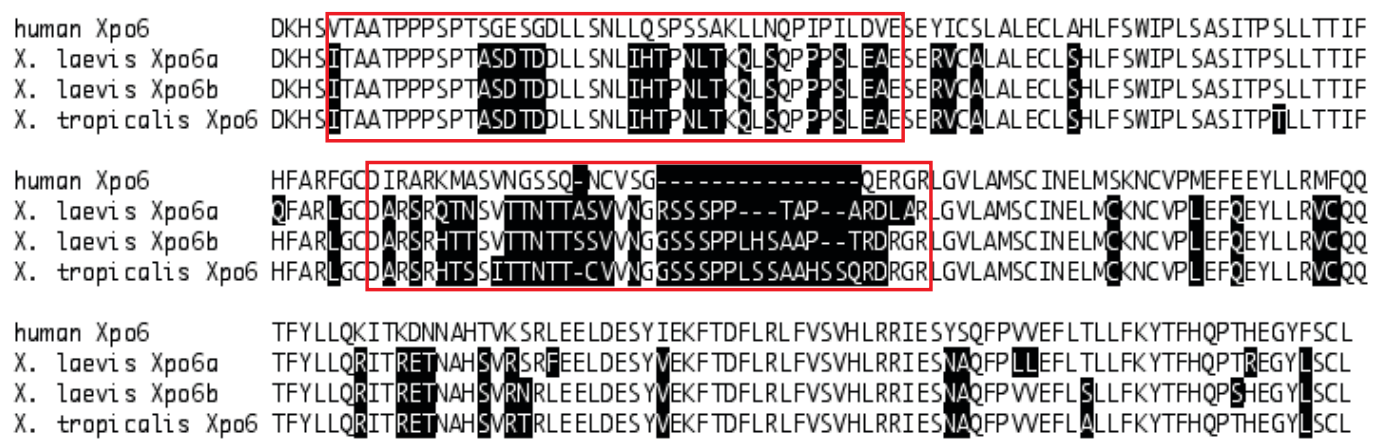

B) Sequence alignment of human, chicken and Dictyostelium Xpo6

human xpo6 LGLLTGILETWDKHSVTAATPPPSPTSGESGDLLSNLLQSPSSAKLLNQPIP ILDVESEYICSLALECLAHLFSWIPLS gallus gallus Xpo6 LGLLTGILESIMDKHSVTAATPPP SPTSGESGDLLSSLLQSPSAAKLLNQPIPILDTDSEY ICSLALECLAHLFSWIPLS D. fasci culatum Xpo6 IQVLTTSLNQLDRTSP-_...-

D. purpureum Xpo6 IGVLSETLNQLFD DNAEKKFK--.----NANLLAFQVGSPDTNTYTGS----FSAETKIITKAVFDALLSYFSWMPLN

human xpo6 ASITPSLLTTIFHFARFGCDIRARKMASWNGSSQNCVSGQERGRLGVLAMSCINELMSKNCVPMEFEEYLLRMFQQTFYL gal lus gallus Xpo6 IISITPSLLTTIFHFARFGdDTRVRKMSSWGSSQNVGLGAERGRLGVLAMSCINELMSKNCVPMEFEEYLLRMFQQTFYL

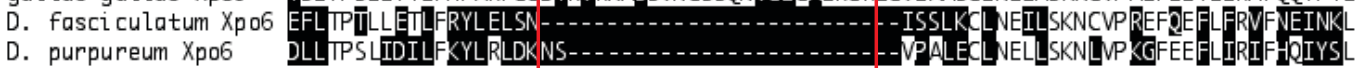

loop regions 1 and 2

Figure 3-27 Sequence conservation of Xpo6 in different species

A) Alignment of Xpo6 sequences from human, X. laevis and X. tropicalis. The predicted loop regions were shown in red boxes. Note the low conservation in these regions compared to the rest of the sequences. Also there is an insertion in the loop 2 regions in frog sequences. B) Alignment of Xpo6 sequences from human chicken and D. purpureum and D. fasciculatum. The predicted loop regions were shown in red boxes. Note the missing sequences in slime mold Xpo6 versions, corresponding to the predicted loop regions. Xpo6 from chicken is highly similar to human Xpo6 throughout the entire sequence.

Using shorter versions of a protein is a frequently used approach in crystallization. Another approach is using proteins from thermophilic organisms. Proteins from thermophiles are evolved to withstand high temperatures, and are therefore more rigid in ambient or low temperatures used for crystallization, compared to their mesophilic counterparts. Since NTRs are only present in eukaryotes, thermophilic fungus Chaetomium thermophilum is the favorite choice for NTR crystallization studies (Monecke et al., 2013). However, Xpo6 being absent in fungi, this species is not a likely solution for us. We therefore decided to use Xpo6 from species with slightly higher body temperatures than humans. Birds with an average $41^{\circ} \mathrm{C}$ body temperature were the first choice. We decided to use Xpo6 from chicken, and would also like to test whether chicken Xpo6 works better with chicken skeletal actin. We expressed and purified Xpo6 from chicken (ggXpo6), D. purpureum (dpXpo6) and D. fasciculatum (dfXpo6) and tested them in a complex formation assay with RanGTP and profilactin in comparison to human Xpo6. The binding assay was performed as described in 3.5.1. We also used Xpo6 from Xenopus laevis as another positive control. ggXpo6 was indistinguishable from human and Xenopus Xpo6 in terms of 
complex formation. However, neither dpXpo6 nor dfXpo6 was bound to profilactin in this setup (Figure 3-28-A). Before giving up hopes on our new species, we wanted to test them on a phenyl sepharose matrix that is now known to increase the stability of the actin export complex.

A

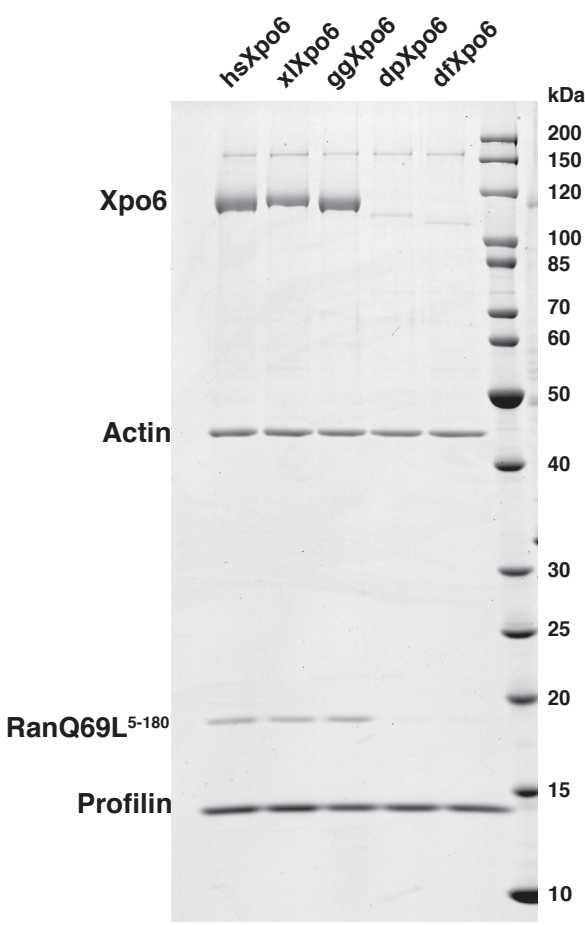

B

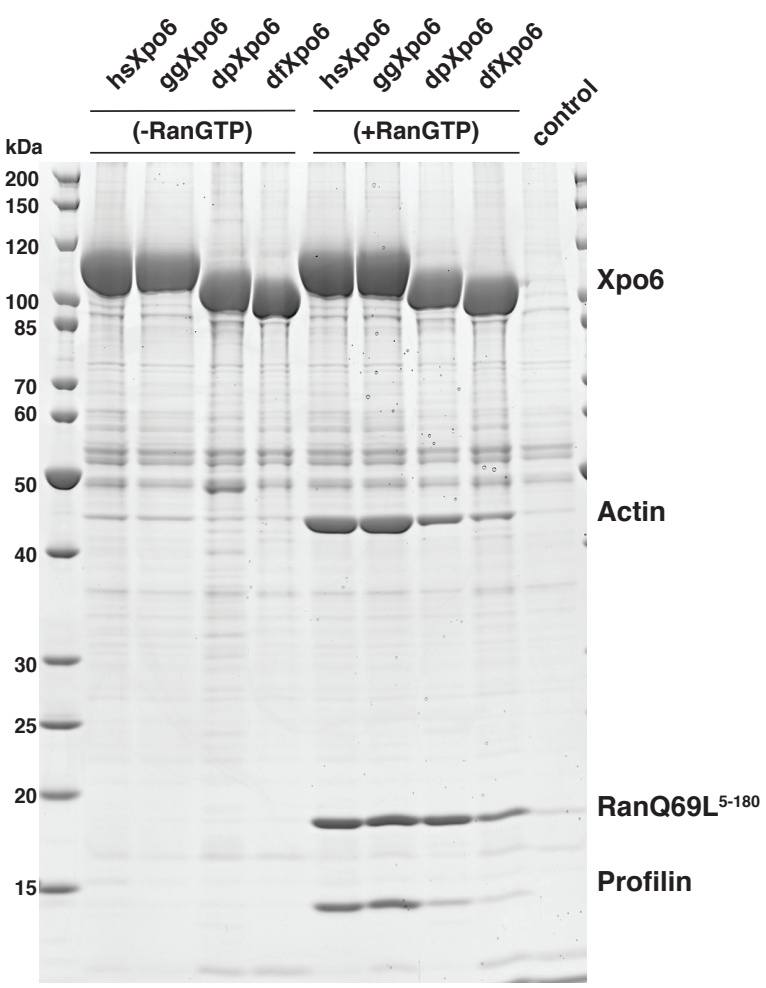

Figure 3-28 Export complex formation with new Xpo6 species

Xpo6 from human, frog, chicken and slime mold were compared for their ability to form an export complex. The buffer for each binding reaction was the same: $10 \mathrm{mM}$ Tris $\mathrm{pH} 7.5,20 \mathrm{mM} \mathrm{NaAc}, 1 \mathrm{mM}$ DTT A) Complex was immobilized through profilin, and the amount of Xpo6•RanGTP bound to profilactin was used to judge the complex stability. Experimental setup was identical to Figure 3-26. Note that human frog and chicken were similar, whereas the Xpo6 from slime molds did not form a complex. B) Complex formation was tested on phenyl sepharose matrix. Experimental setup was identical to Figure 3-4. The Note that human and chicken Xpo6 are similar, whereas the Xpo6 from slime molds bind weaker to RanGTP and profilactin. However, the binding is specific and significantly higher than background as shown by the control: without any Xpo6.

Indeed both dpXpo6 and dfXpo6 bound RanGTP and profilactin. The binding was weaker compared to human and chicken Xpo6, but it was clearly stronger than background control (Figure 3-28-B). The difference between a weak binding and no binding is very significant. We can say that Xpo6 from these two evolutionary distant species still retain the ability to bind Ran, actin and profilin from human. The binding may be enhanced if the binding partners are chosen form the same species. We will use these new Xpo6 constructs for crystallization studies of the free Xpo6, as well as for the crystallization of the actin export complex. 


\subsubsection{Crystallization of the actin nuclear export complex}

We used the purified actin export complex (Figure 3-13) for crystallization trials. The sample was concentrated to $15 \mathrm{mg} / \mathrm{mL}$, with good polydispersity values $(12 \%)$. As a control we have used Xpo6 $\bullet$ RanGTP complex at $15 \mathrm{mg} / \mathrm{mL}$ concentration, purified in the same buffer. Using the robotic system we have screened a broad range of conditions using commercially available sparse matrix screens (6.1.6). For each screen we have prepared plates for two different temperatures $\left(4^{\circ} \mathrm{C}\right.$ and $\left.20^{\circ} \mathrm{C}\right)$. We identified several conditions, where promising crystalline growth was observed. Figure 3-29 shows images of some of the initial hits. Interestingly, for the actin export complex all hits were from plates at $20^{\circ} \mathrm{C}$. in their counterparts at $4^{\circ} \mathrm{C}$, no crystals have been observed. For the conditions termed as initial hits, Xpo6•RanGTP controls did not show any crystalline growth.

A) $10 \%$ PEG $6000,10 \mathrm{mM} \mathrm{MgCl}-20^{\circ} \mathrm{C}$
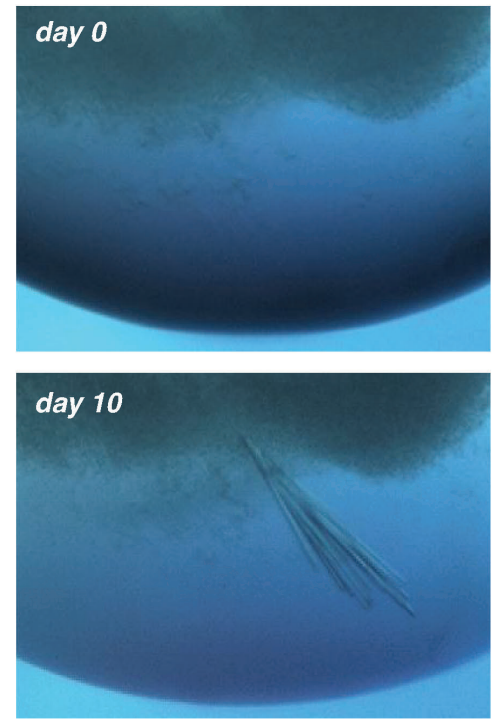

B) $15 \%$ PEG $1500-20^{\circ} \mathrm{C}$
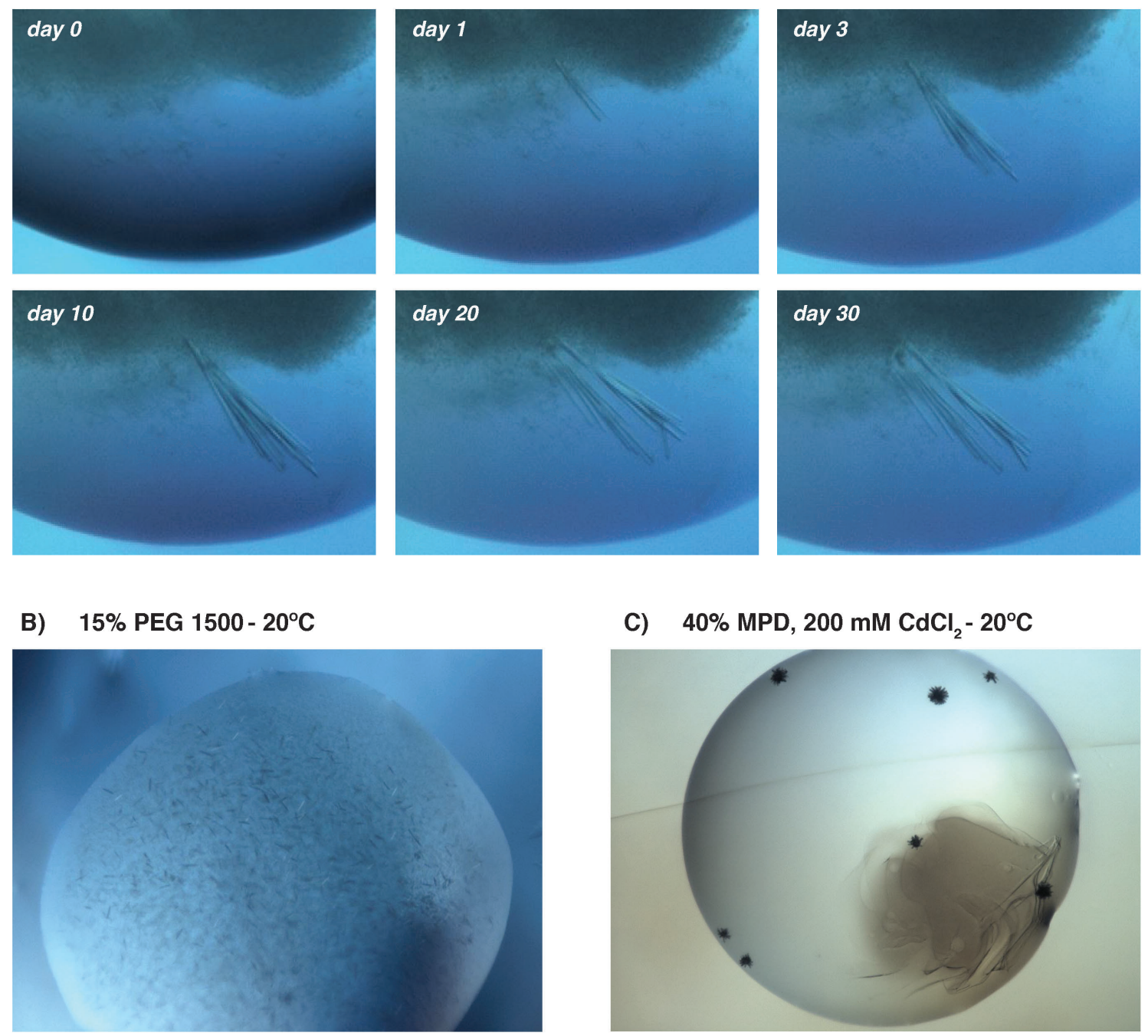

C) $40 \% \mathrm{MPD}, 200 \mathrm{mM} \mathrm{CdCl}-20^{\circ} \mathrm{C}$

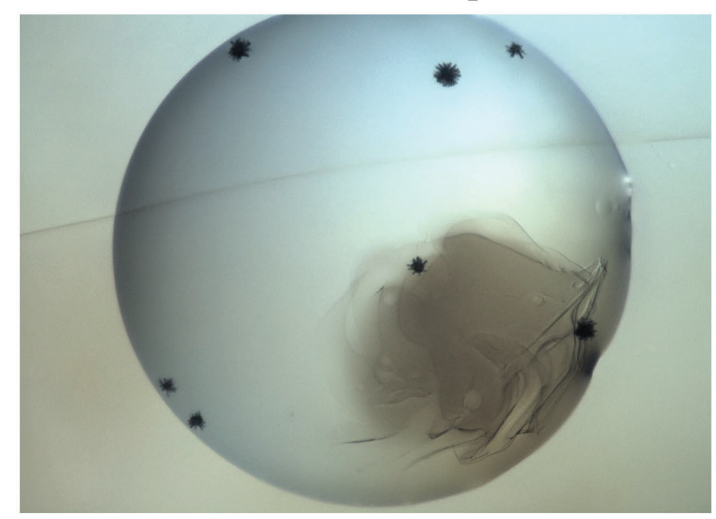

Figure 3-29 Initial hits for actin nuclear export complex crystallization

The crystallization conditions and temperature are indicated. A) needle crystals appear at day one and continue to grow until day 30. The crystals are indicated with the red boxes. B) numerous small needles appear on day 3, but do not grow further. C) Dark precipitates appear on day one and grow outwards as needles. 
It was an interesting observation that all the initial hits were conditions that did not contain any salt. Considering the ionic sensitivity of the actin export complex, this was a positive indication. Two of the initial hits were especially promising. In one condition (10\% PEG $6000,10 \mathrm{mM} \mathrm{MgCl}_{2}$ ) a small needle crystal appeared in day 1, and continued growing as a bunch of needles until day 30. In another very minimalistic condition (15\% PEG 1500), numerous small needles appeared at day 3 , but they did not grow further. Another possible hit is an alcohol-based condition, which also contains a high concentration of heavy metal salt (40\% MPD, 200mM $\mathrm{CdCl}_{2}$ ). In this condition, dark precipitates appeared on day 1 , and continued growing radially in a way that resembles crystalline growth. It is possible, however, that those are crystals of the heavy metal salt.

We prepared refinement screens for conditions based on PEG 6000 and PEG 1500. For the first hit, we kept the $10 \mathrm{mM} \mathrm{MgCl}$ fixed, whereas we varied PEG 6000 concentration from $0-25 \%$ over 96 wells of the screening plate. For the other refinement screen we scanned PEG 1500 concentrations of $0-20 \%$. Unfortunately, we could not identify a better crystallization condition, nor we could reproduce the initial observations.

Nucleation is a rare event, and it is possible that it can not be reproduced. But nucleation step could be by-passed with a technique called seeding. In seeding method, sub-optimal crystals are crushed to form microcrystals, which act as nuclei for the next round of crystallization. We wanted to use the needles obtained in the initial screen $(10 \%$ PEG $6000,10 \mathrm{mM} \mathrm{MgCl}_{2}$ ) as seeds for our refinements. The needle crystals were taken out from the drop and a seed stock solution was prepared (see 6.2.13.4). While preparing new reservoir solution for the manual seeding experiments, we realized that the $\mathrm{pH}$ of the manually prepared PEG 6000 solution was very acidic ( $\mathrm{pH} 4.3)$. Although the commercial screen did not have any buffer in the condition, they might have $\mathrm{pH}$ adjusted the PEG 6000 solution, which was not explicitly described in the manuals. Considering that the $\mathrm{pH}$ might have been the reason why we did not see any crystals in our refinements, we added 100 $\mathrm{mM}$ Tris $\mathrm{pH} 7.5$ to the solution. The $\mathrm{pH}$ was then manually confirmed. Using the seed stock dilutions and the newly prepared reservoir solution (10\% PEG $6000,10 \mathrm{mM} \mathrm{MgCl}_{2}$, $100 \mathrm{mM}$ Tris pH 7.5) we prepared our seeding drops. Serial dilutions of the seed stock with reservoir were mixed with protein $(15 \mathrm{mg} / \mathrm{mL})$ in $1: 1,1: 2$ ratios and sealed in small chambers with reservoir at room temperature. Bunches of needles appeared in almost all dilutions of the seed stock within 1-2 days. On the third day, needles appeared also in the control drops without seed stock, but only reservoir. A characteristic bowtie shape was 
observed: needles from one nucleus usually grew towards both directions radially. Figure 3-30 shows the crystals obtained in manual drops.
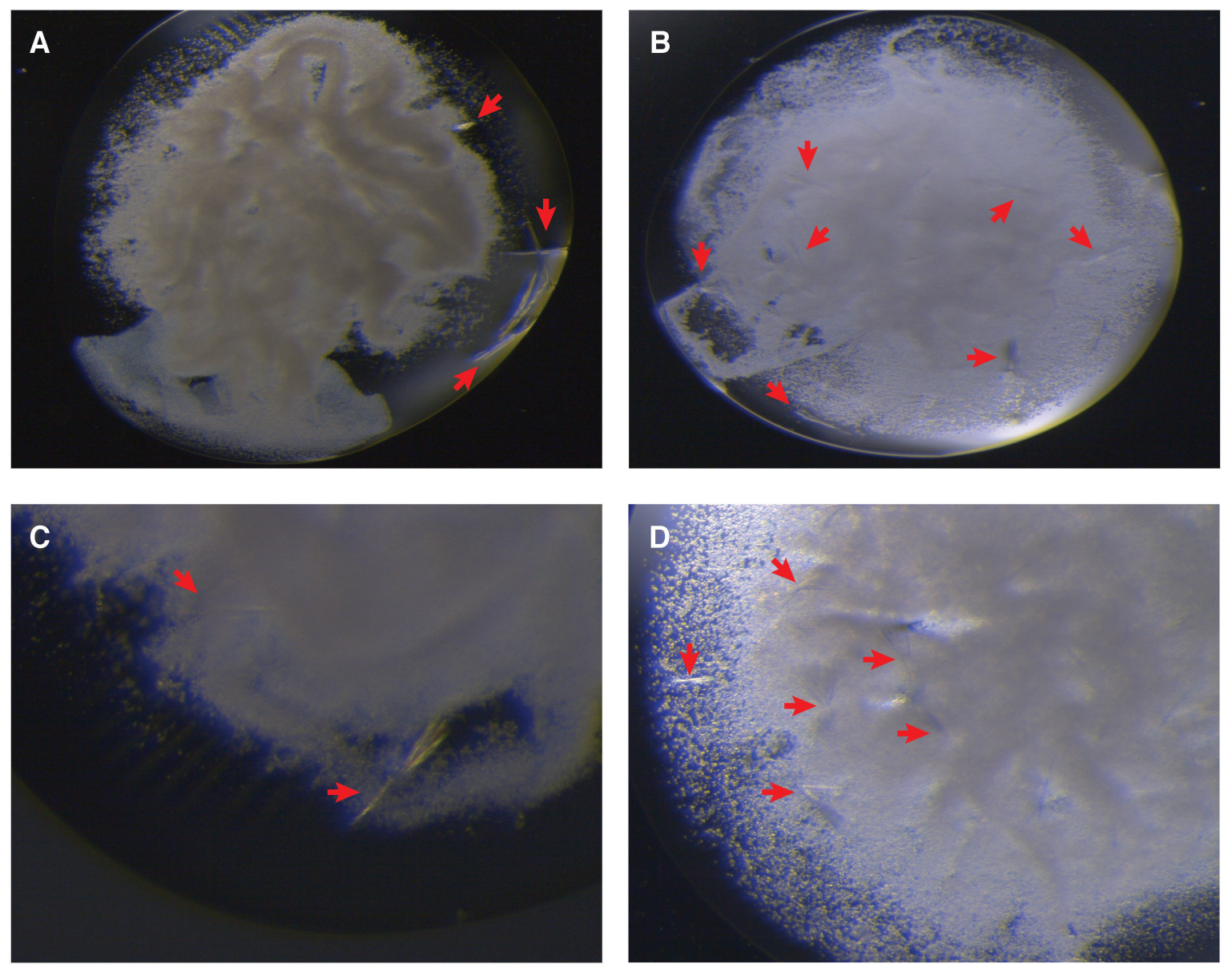

Figure 3-30 Reproduction of the actin nuclear export complex crystals

The needles from the initial hit $\left(10 \%\right.$ PEG $\left.6000,10 \mathrm{mM} \mathrm{MgCl}_{2}\right)$ were used to prepare seeds, which were used in the manual crystallization drops with the following crystallization solution: $100 \mathrm{mM}$ Tris $\mathrm{pH} 7.5,10 \%$ PEG $6000,10 \mathrm{mM}$ $\mathrm{MgCl}_{2}$. In drops with various seed concentrations (A, C, D), as well as in drops without seeds (B), needle shaped crystals could be reproduced in several days at room temperature.

The crystals were reproducible with and without seeding, and there were no visible improvements in the crystal shape due to seeding. We therefore wanted to know what the crystals were made of: was it the tetrameric export complex or any of the sub-complexes? In order to investigate the content of the crystals, we removed them from the crystallization drop with a nylon loop, and washed them in a fresh drop of reservoir in order to prevent carry over of soluble protein from the solution. Finally the crystals were placed in $5 \mu \mathrm{L}$ of fresh reservoir in a tube. Two drops were harvested as described and several crystals from each drop were combined and analyzed by SDS PAGE. As a control, $1 \mu \mathrm{L}$ of protein sample was loaded to the gel. Figure 3-31 shows the SDS PAGE analysis of the crystals from two separate drops. As can be seen, all four proteins were present in both crystals. 
We have managed to crystallize the full actin nuclear export complex from human, and that in a very minimalistic condition: $10 \%$ PEG $6000,10 \mathrm{mM} \mathrm{MgCl}_{2}, 100 \mathrm{mM}$ Tris $\mathrm{pH}$ 7.5. For sure the crystals are still not optimal for diffraction analysis and require further optimizations. But it was very important to show that the full-length human actin nuclear export complex can be crystallized, despite its ionic sensitivity, the low affinity between its sub-complexes and even the possible inhomogeneity in the crystallization sample. In the end the crystallization process itself has been the ultimate purification in a mixed population between the full complex and sub-complexes.

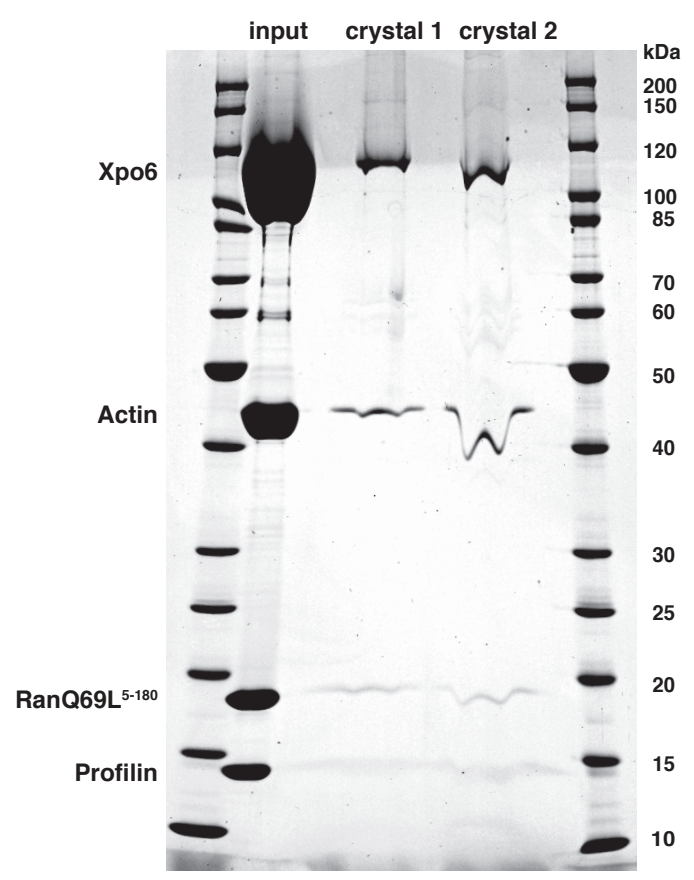

Figure 3-31 SDS PAGE analysis of the actin nuclear export complex crystals

The needle crystals from the manual drops were fished, washed in fresh reservoir and resuspended in reservoir solution with SDS buffer. The content of the crystals were analyzed with SDS-PAGE, and a microliter of the protein sample was loaded as a reference. Crystals from two different drops are shown here to contain all four proteins of the export complex. The strange running behavior of the proteins is probably a result of the PEG 6000 in the loaded sample. 


\section{Discussion}

\subsection{Actin isoforms and the complex stability}

We have shown that the muscle $\alpha$ actin was not a very good substrate for export complex formation in vitro. This was mainly due to the lower affinity of $\alpha$ actin towards profilin. The lower affinity of $\alpha$ actin towards profilin than its cytoplasmic counterparts $(\beta / \gamma)$ has been reported previously (Larsson and Lindberg, 1988; Ohshima et al., 1989; Vinson et al., 1998; Kinosian et al., 2000). Profilin is a multi-functional protein in actin cytoskeleton dynamics. It has a major role in the ATP loading of actin monomers, and their addition to the barbed end of the actin filaments (Sohn and Goldschmidt-Clermont, 1994). Since the cytoplasmic actin filaments are more dynamic and have a higher turnover rate than the sarcoplasmic actin bundles, it makes perfect sense that the cytoplasmic $(\beta / \gamma)$ actin isoforms show higher affinity towards profilin, which assists the re-polymerization of the recycled monomers. Also, $\alpha$ actin was shown to polymerize more readily than $\beta$ actin, especially at low temperatures (Gordon et al., 1977). Actin in its filamentous form is not accessible for profilin or Xpo6. It was actually shown that Xpo6 efficiently exports $\alpha$ actin from the Xenopus oocyte nuclei (Bohnsack et al., 2006). This is, however, not exactly controversial with our findings, considering that many other cellular factors can stabilize the export complex in vivo.

For all the obvious benefits, we decided to use an actin isoform with higher affinity for profilin, and hence for the complex. Using HeLa cytoplasmic extract as a source for actin was the immediate choice. Department of cellular biochemistry at the MPI for biophysical chemistry has an established protocol for preparing nuclear and cytoplasmic HeLa extracts and they have generously supplied us with large amounts of cellular extract. Cytoplasmic actin is a mixture of two isoforms; $\beta$ actin and $\gamma$ actin are found in varying ratios in different tissues (Herman, 1993; Perrin and Ervasti, 2010). The isoforms can be separated via hydroxyapatite chromatography (Segura and Lindberg, 1984), however the use of this resin is cumbersome. At the beginning we were concerned whether the mixed isoforms would be a good substrate for crystallization. However, $\beta$ and $\gamma$ actins are almost identical, except for four very conserved amino acid changes at the extreme $\mathrm{N}$-terminus of the protein. There are also previous studies reporting successful crystallization of $\beta$ and $\gamma$ actin 
isoforms (Carlsson et al., 1976). For this reason we decided to use the cytoplasmic actin without further separation of the isoforms.

\subsection{Stable Xpo6•RanGTP interaction in the absence of the cargo}

Xpo6 binds RanGTP strongly, as shown in section 3.3, even in the absence of its cargo. This indicates a cargo recognition mechanism that is different than CRM1 and CAS, which have both very low affinity towards RanGTP in the absence of their respective cargos (Fornerod et al., 1997; Kutay et al., 1997). A strong affinity towards RanGTP is a feature that is characteristic to importins. RanGTP, due to this high affinity, is able to disassemble import complexes when they arrive in the nucleus. This brings up the question whether Xpo6 might have an import function parallel to the actin export. There are already known bidirectional NTRs, such as the importin 13 (Mingot et al., 2001) and exportin 4 (Lipowsky et al., 2000; Gontan et al., 2009). Also Msn5 in yeast is known to export one set of cargos including transcription factors and cyclin inhibitors, while importing RPA, an important protein in DNA replication and repair (Yoshida and Blobel, 2001). It is possible that Xpo6 is indeed specific for only one single task, but it might as well have other functions in import or export. Relatively high background in the pull-down experiments (Stuven et al., 2003) might have been the reason, why no other cargo for Xpo6 was identified so far. We can now overcome this problem by using the new affinity tag \& matrix systems that are developed in our lab, which show almost no unspecific binding in the cellular extracts. Also, if one is looking for potential import cargos, using a nuclear extract for the pull-down experiments will increase the likelihood of identification. For identifying potential export cargos beside actin, one would rather use a cytoplasmic extract that is actin-depleted, in order to be able to identify less abundant potential cargos.

\subsection{Crystallization of human Xpo6}

While setting up the crystallization screens for Xpo6, we saw that the concentrated Xpo6 $(25 \mathrm{mg} / \mathrm{mL})$ showed some precipitation after standing on ice overnight. The sample was ultra centrifuged to remove precipitates and the pellet was observed under a light microscope. To our great surprise, we saw that the precipitates were actually small needle crystals, some of which we could also detect in the newly pipetted crystallization plates. This was a good indication that free Xpo6 had a potential to crystallize. In accordance with this, we identified several different conditions, where Xpo6 showed some crystalline growth. All identified conditions had polymers as precipitants such as polyethylene glycol 
(PEG), Jeffamine ${ }^{\circledR}$ and a variety of Sokalan ${ }^{\circledR}$ polymers. PEG as precipitant seemed to be preferred at rather low concentrations for crystallization. The identified conditions were reproducible, even after 2 freeze/thaw cycles of the sample. Some protein samples may not tolerate a freeze/thaw cycle, and thus can only be crystallized if they are freshly prepared. That Xpo6 may be crystallized after freeze/thaw is a great advantage, enabling us to prepare protein in large scale, freeze in aliquots and use for crystallization whenever necessary. So far, the diffraction of the Xpo6 crystals was limited to $7.4 \AA$. The mosaicity was low, indicating proper packing of crystals; and the completeness of the data was $100 \%$ up to the resolution limit. We did not observe a trend in the resolution of crystals relative to the $\mathrm{pH}$, salt and precipitant concentration of the crystallization conditions. The space groups for crystals from two different conditions (PEG MME 2000 and Sokalan CP 12S) were found to be the same (trigonal $\mathrm{P} 3_{1} 21$ ). Unit cell dimensions were quite large: 203, 203, $258 \AA$. Considering the average dimensions of NTRs (about $5 \times 10 \mathrm{~nm}$ ) (Monecke et al., 2009; Bono et al., 2010), many Xpo6 molecules are expected to be found within the unit cell.

In order to improve resolution of the Xpo6 crystals, we tried to identify and remove the possible flexible loops from the protein. Combining the limited proteolysis experiments, secondary structure predictions and the sequence conservation analysis two possible loop regions were identified, which we truncated from the full-length construct. Removal of loops would make the protein more compact and hence might enhance the solubility during recombinant production. We did not observe an increase in the solubility for the constructs. Also, a greater portion of the truncated Xpo6 versions eluted from the gel filtration as high molecular weight assemblies. These soluble aggregates might indicate a sub-optimal folding of the truncated Xpo6. While removing internal loops, one has to leave enough amino acids to properly bridge the adjacent folded domains. We created several truncation versions, with up to 4 amino acids as linkers between the two neighboring helices. All the loop truncations did bind to RanGTP and profilactin in the binding assays, indicating that the removal of the loops did not interfere with the function. However, we could not produce any crystals with the three of the Xpo6 truncations we tried in crystallization screens. This could simply be a result of the crude screening for the optimal linker length. A tension created in the molecule because of a too-short linker may result in positional shifts in the potential crystal contact points of the molecule. By screening the linker length in more precision, one could reach a perfectly trimmed Xpo6 for crystallization. These 
loops might not have direct functions in cargo binding, but they might otherwise be related to the Xpo6 function. They may for example, interact with the FG nucleoporins and be involved in the docking or in the NPC passage of Xpo6. These possibilities can be tested by hydrogel permeation assays (Frey and Gorlich, 2007), or by export assays with permeabilized HeLa cells.

Along with the manual truncations of Xpo6 we have chosen Xpo6 from other species as alternative crystallization substrates. Xpo6 from chicken was selected because of the slightly higher body temperatures of these animals. Proteins that are adapted to higher temperatures are preferred for crystallization in order to reduce flexibility. Proteins would be more rigid with the increasing difference between the optimal temperature and the crystallization temperature. Xpo6 species from two slime molds were chosen, because of their overall smaller size and their missing sequences at the two identified loop regions.

Another approach that we will try for improving crystallization is the use of nanbodies. Nanobodies are small $(15 \mathrm{kDa})$ and stable single-domain fragments of the original heavy chain-only antibodies that naturally occur in camelids (Muyldermans, 2013; De Meyer et al., 2014). They retain the full antigen-binding capacity of the antibodies, and have several other features that make them useful aids for improving crystallization. They bind the target with nanomolar affinity and often recognize epitopes that are less immunogenic or accessible for conventional antibodies. Nanobodies generally bind conformational epitopes and might stabilize flexible regions of proteins in a given position. They also might stabilize the protein-protein interfaces in multi-protein complexes. There are already numerous examples of nanobodies successfully being used as crystallization chaperones (Loris et al., 2003; Korotkov et al., 2009; Lam et al., 2009). Our lab recently established a workflow for the development and purification of nanobodies from alpacas we have at the institute. We have used free Xpo6, Xpo6•RanGTP, and actin nuclear export complex for immunization of an alpaca over the course of one year. We then isolated RNA from the animal for the development of libraries. Next step will be the creation of a library, selection of high affinity binders using phage display system and characterization of the nanobodies. We expect to identify several high affinity binders for Xpo6 and also other complexes that may significantly improve crystallization. 


\subsection{Xpo6 in evolutionary distant species}

Using a shorter version of Xpo6 might be of great advantage for crystallization, but one also has to consider the conservation of function. The Xpo6 from D. purpureum and D. fasciculatum are only $30 \%$ identical to the human protein in sequence. In binding assays described in section 3.9.2.2, we could show that Xpo6 from these evolutionary distant organisms still retain their capability to bind profilactin in a RanGTP dependent manner. However, whether these Xpo6 variants still function in actin export in the host organisms remains to be answered. If one looks at the evolutionary conservation of Xpo6, one can see that it is not as nearly conserved as CRM1 or $\operatorname{Imp} \beta$. One can find Xpo6 in all vertebrates, and in some other chordates. Xpo6 sequences are also found in insects and arachnids, but not in nematodes or other lower animals. Xpo6 is also absent in fungi and plants. However, one finds Xpo6 in slime molds, which are evolutionarily more distant to vertebrates than fungi. The question, when Xpo6 was invented during evolution, is a puzzling one. Whether it was invented independently for the distant branches of the phylogenetic tree, or whether the fungi and the lower animals have lost it can not be determined easily. In order to be able to differentiate between these scenarios, one needs more complete sequence information. There are only two exceptional examples of Xpo6 in nematodes and fungi: Trichinella spiralis, a parasitic worm for pigs, rodents and humans and Batrachochytrium dendrobatidis, a parasitic fungus for frogs and fish. It is likely that these species acquired Xpo6 via horizontal gene transfer from their hosts over the course of time. Therefore, it is tempting to think Xpo6 in slime molds might also be a result of horizontal gene transfer. Slime molds are soil organisms that contribute to the decomposition of organic material and feed on bacteria yeasts and other fungi. Decaying animals in the soil might be a likely route for the horizontal gene transfer. If slime molds acquired Xpo6 through horizontal gene transfer, it would be interesting to see whether Xpo6 still retains its function to export actin from the nucleus.

\subsection{Ionic sensitivity of the actin nuclear export complex}

Our results showed that the actin export complex is made of two sub-complexes: profilactin and Xpo6•RanGTP. These sub-complexes are stable in moderate up to high salt concentrations, respectively. However, the interaction between them is very sensitive to ionic strength of the buffer. We showed that the association between Xpo6•RanGTP and profilactin can completely be abolished by increasing the $\mathrm{NaCl}$ concentration to $100 \mathrm{mM}$. Such high sensitivity to ionic strength can be explained if the interface between the sub- 
complexes is stabilized by ionic interactions. Ionic species in the buffer can interact with the charged residues and disrupt the salt bridges between the sub-complexes. An interesting finding along these lines was that the actin export complex tolerated $\mathrm{NaAc}$ better than $\mathrm{NaCl} . \mathrm{Cl}^{-}$ion might be more effective in disrupting the ionic interactions due to its small size, compared to the much larger acetate ion. We also observed a much lower stability of the actin export complex in potassium phosphate buffers. Interestingly, in this buffer, the complex stability also showed a greater $\mathrm{pH}$ dependency. At lower $\mathrm{pH}$, complex was less stable, whereas the stability gradually increased with increasing $\mathrm{pH} . \mathrm{pH}$ variations can also change the ionization states of the amino acid side chains, which in turn will have an effect on the ionic interactions. When our observations are taken into account, it is highly likely that the interface between Xpo6 and actin is maintained by salt bridges.

In addition to the ionic sensitivity, the overall affinity profilactin for the complex seems to be low, implied by the tailing of the complex peak during MALS analysis gel filtration. Also the calculated Mw of $177 \mathrm{kDa}$ for the peak is lower than the expected $\mathrm{Mw}$ for the full-length complex, $205 \mathrm{kDa}$. The discrepancy (28 $\mathrm{kDa}$ ) corresponds to the half of the $\mathrm{Mw}$ of profilactin $(57 \mathrm{kDa})$ and can be explained if the peak population consists of the full export complex and Xpo6•RanGTP. Despite the low affinity, we can say that the on-rate for the complex formation is far greater than the off-rate. We can conclude this from the observation that longer incubation times of the sub-complexes increased the complex yield during our experiments.

\subsection{Phenyl sepharose stabilizes the actin nuclear export complex}

Ionic strength at physiological conditions is already above the limit of what the actin nuclear export complex tolerates in vitro $(100 \mathrm{mM} \mathrm{NaCl})$, yet the complex exists and is functional in vivo. There must be other factors stabilizing the export complex in the cell. Also the molecular crowding within the cell might stabilize the complex. Most importantly, the central channel of the nuclear pore complex interacts with NTRs via the FG repeats of nucleoporins (Ribbeck and Gorlich, 2002). Binding to FG repeats might stabilize Xpo6 in its cargo bound conformation. It was therefore a very interesting observation that the actin export complex was not nearly as sensitive to ionic strength when it was assembled on phenyl sepharose matrix. Phenyl sepharose matrix is known to bind NTRs with high affinity (Ribbeck and Gorlich, 2002). It is possible that phenyl sepharose mimics the NPC environment by occupying multiple FG binding pockets of Xpo6 simultaneously. We tried to reproduce this effect by the addition of phenyl- or 
benzyl- group containing compounds (phenylmalonate, benzylmalonate, sodium benzoate, phenylacetate, etc) in the complex formation reactions. The addition of such compounds did not improve the stability. It is likely that a stabilizing effect is a result of the occupation of multiple binding pockets in a close proximity, which can not be achieved by single phenyl groups.

Another observation that supports this idea came from the thermofluor assays. As shown in section 3.8, we obtained fluorescence signals before the start of the melting, indicating that the fluorophore interacts with Xpo6 even when it is not denatured. However, the more interesting result of this experiment was that the fluorescence signal at $30^{\circ} \mathrm{C}$ for the export complex was significantly higher compared to Xpo6 and RanGTP. This could indicate that upon cargo binding, Xpo6 undergoes conformational changes that further expose the hydrophobic FG-binding pockets. This observation is in accordance with the stabilizing effects of the phenyl sepharose on the export complex.

\subsection{Topological analysis and crystallization of the actin nuclear export complex}

We performed several binding experiments to test the accessibility of profilactin within the export complex. With this we wanted to understand which part of the profilactin molecule might be in direct contact with Xpo6 and possibly RanGTP. The poly-L-Proline binding pocket of profilin was the first region to look at. We could indeed immobilize the full export complex to a Poly-Pro sepharose matrix via the binding pocket of profilin. For this, there should be no steric hindrances and profilin should be freely accessible. Poly-L-Pro binding pocket is localized opposite to the actin-binding interface, and it is therefore likely that profilin faces rather outwards in the complex, whereas actin is directly contacted by Xpo6. Mena, VASP and mDia are formins known to interact with profilin via their proline rich sequences. They were also identified in the initial Ran dependent Xpo6 pull-downs (Stuven et al., 2003). In the light of this finding, one can also speculate that these proteins bind to the actin export complex via the accessible poly-L-pro binding pocket of profilin during the pull-down experiment.

DNaseI binding loop of actin is localized in the subdomain 2, on the opposite side of the profilin binding interface of actin (Figure 4-1). Our results showed that DNaseI efficiently dissociates Xpo6 $\bullet$ RanGTP and bind profilactin, when incubated with a pre-formed export complex. However, it is not straightforward to interpret the result of the experiment. There 
are two possibilities, which may lead to the same outcome. One possibility is that the Dloop of actin is accessible in the context of the export complex, yet the binding of DNaseI to D-loop is sterically not compatible with Xpo6 binding. In this scenario, DNaseI can efficiently displace Xpo6, owing to the fact that its affinity to actin higher than that of Xpo6. Another possibility is that the D-loop contacts Xpo6, and is not accessible for DNaseI. In this case, DNaseI can only bind actin, when Xpo6 6 RanGTP dissociates from actin as a result of the off-rate. DNaseI binding would make actin inaccessible for the rebinding of Xpo6, hence DNaseI will slowly replace Xpo6. Dissociation of Xpo6 $\bullet$ RanGTP from profilactin was also seen in the negative control that was incubated with buffer for one hour. With the information derived from this experiment, we can not discriminate between the two scenarios, but we can say that Xpo6 possibly contacts actin near its pointed end, leaving profilin mostly accessible for other binding partners.

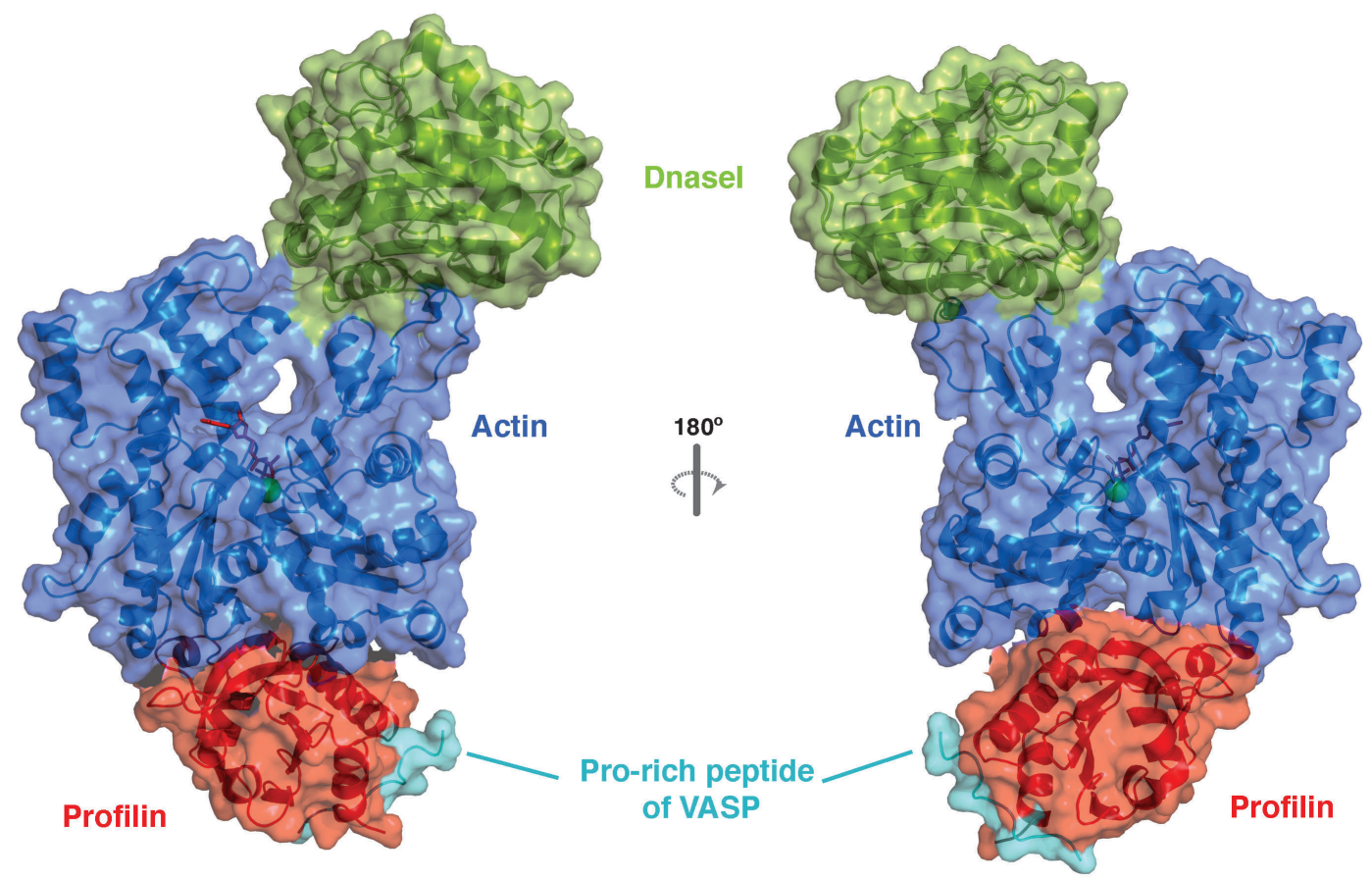

Figure 4-1 Mapping of the binding sites on the actin monomer

Profilin and DNaseI are represented on a single actin monomer. Also, the Pro-rich peptide of VASP is shown in the PolyPro-binding pocket of profilin. In our experiments we found that this pocket was available in the context of the complex, but the binding of DNaseI to profilactin was incompatible with Xpo6 binding. Image is prepared using PyMol using data for actin and DNaseI from the structure with PDB-ID: 2A42 (Chereau et al., 2005), for profilin and the bound Pro-rich VASP peptide from the structure with PDB-ID: 2PBD (Ferron et al., 2007). 
Actin export complex has proven itself not to be an easy target for crystallization. We optimized our purification conditions in the light of our findings to obtain a complex that is mostly stable over gel filtration. Nonetheless, the final product of complex purification may contain a slight heterogeneity due to the dissociation of the complex. An alternative approach to gel filtration is the purification of the complex via two affinity tags. Orthogonal proteases have been established in our lab for sequential affinity purifications, which can be used for purification of stoichiometric complexes (Frey and Gorlich, 2014). Our experiments show that the $\mathrm{N}$-terminus of profilin and N-terminus of Ran are accessible in the context of the assembled complex and can be tagged for a sequential pulldown from each sub-complex.

Despite the possible inhomogeneity of the crystallization sample, we managed to identify a crystallization condition, where all four components of the actin nuclear export complex crystallized. This is a very crucial step forward, proving the potential of the complex to crystallize and showing that crystallization can be the best purification step itself. From this point on, we can only further improve the current condition and the crystals. Besides the usual optimization workflow of crystallization, we can implement the improvements from Xpo6 crystallization to the complex, such as the optimally truncated Xpo6 constructs, or new Xpo6 species. Furthermore, we expect to find nanobodies against the export complex in our nanobody library. If we can identify a nanobody that stabilizes the interface of the two sub-complexes, this will greatly improve the purification and crystallization of the export complex. With the nanobodies, it is even possible that we find many other crystallization conditions for the complex.

\subsection{Exportin 6 and the IRM actin mutants}

We tried to establish an experimental strategy, in order to test our initial hypothesis that Xpo6 fails to recognize the mutant actins seen in IRM cases. The mutant actins have to be produced recombinantly. The most straightforward method is the in vitro translation (IVT) of the wild type and mutant actins with radiolabeled methionine. We designed an experimental set up as follows. Wild type actin and several IRM mutants are translated with rabbit reticulocyte lysate, and the translation reaction is used as a source for actin in our standard complex formation experiments (3.5.1). The actin (wt and mutants) in the input, flow through and in the Xpo6-bound fractions are detected by autoradiography. However, we failed so far to show a RanGTP dependent binding even for the positive control, the wild type $\alpha$ actin. The major problem was the low affinity of $\alpha$ actin towards 
Xpo6. Also the cytoplasmic actin in the rabbit reticulocyte lysate acted as a competitor for Xpo6 binding. We could show Ran-dependent binding of actin to Xpo6, but this was only cytoplasmic actin from the lysate, as judged by the autoradiography.

We will have to use another approach for testing the export capabilities of the IRM mutants. The best alternative is to use the Xenopus oocyte system for an in vivo export assay, which has been successfully used to show that Xpo6 can export different isoforms of actin and yeast actin (Bohnsack et al., 2006). In this experimental setup, radiolabeled actin mutants are produced with IVT, and the translation reaction is microinjected into the nucleus of Xenopus oocytes. Since the oocytes lack Xpo6, actin can only be exported if injected together with Xpo6. The localization of the radiolabeled products is determined by the dissection of the oocytes and the isolation of the individual nuclei. If our hypothesis holds true, we should see that the wild type $\alpha$ actin is exported, whereas the mutants fail to do so. However, Xpo6 will not only export the injected actin, but also the complete intranuclear actin meshwork of the oocytes. This represents the major challenge in this experimental setup. When the nuclear actin is exported, the nuclei become very fragile making the isolation a very tedious task, which is why we wanted to establish an alternative system in the first place. Despite the handling skills and the time it requires, this approach is very likely to give us definite answers about the export behaviors of the IRM actin mutants. 


\section{Outlook}

In this project we were able to biochemically characterize the actin nuclear export complex in detail. We describe the formation of a stable in vitro complex, by analyzing its limitations and drawbacks. Moreover, we describe the conditions for successful crystallization not only for the actin nuclear export complex, but also for human Xpo6 in its free form. With these findings, we set the foundation for structural understanding of cargo recognition by Xpo6. The identified crystallization conditions as well as the crystallization substrates will be improved as described in the result and discussion sections. Obtaining structural information of Xpo6 in its free form, of the Xpo6•RanGTP complex and the complete export complex will provide us with a complete picture of the actin nuclear export. Analyzing the nuclear export of IRM actins by Xpo6 will help us to understand the mechanisms underlying intranuclear rod myopathies. 


\section{Materials and Methods}

\subsection{Materials}

\subsubsection{Chemicals}

All laboratory reagents were purchased from Calbiochem (San Diego, CA, USA), GibcoBRL-Life Technologies (Paisley, UK) Merck (Darmstadt, Germany), MoBiTech (Göttingen, Germany) Pharmacia (Uppsala, Sweden), Promega (Madison, WI, USA), Qiagene (Hilden, Germany), Roche (Mannheim, Germany), Roth (Karlsruhe, Germany), Sigma-Aldrich (St. Louis, MO, USA).

\subsubsection{Instruments}

Table 6-1 Laboratory equipments used in this study

\begin{tabular}{|ll|}
\hline Instrument & Manufacturer \\
\hline EenePulser $^{\mathrm{TM}}$ & BIORAD, Burlington, USA \\
\hline Eppendorf biophotometer & Eppendorf, Germany \\
\hline Sonifier 450 & Branson, UK \\
\hline UV-table & Benda Laborgeräte, Wiesloch \\
\hline Perfection V700 Photo Scanner & Epson \\
\hline Incubator/Climo-shaker ISF1-X & Kuhner Shaker \\
\hline Thermomixer comfort & Eppendorf, Germany \\
\hline Thermo NanoDrop 2000C & peqLab, Germany \\
\hline SensoQuest lab-cycler & SensoQuest, Göttingen \\
\hline Äkta-Explorer, Äkta-Purifier & Pharmacia, Uppsala, Sweden \\
\hline DynaPro NanoStar & Wyatt Technology, Dernbach, Germany \\
\hline MiniDAWN & Treos \\
\hline 1260 Infinity Quaternary LC system & Wyatt Technology, Dernbach, Germany \\
\hline Cartesian 8-channel nanodispenser & Agilent Technologies, Waldbronn, Germany \\
\hline Freedom Evo liquid handling robot & Genomic solutions, Digilab Inc, MA, USA \\
\hline Rock Imager automatic imaging system & Tecan Group Ltd., Männedorf, Switzerland \\
\hline Leica MZ6 microscope & Formulatrix Inc, Walthom, MA, USA \\
\hline
\end{tabular}

Table 6-2 Centrifuges and rotors

\begin{tabular}{|lll|}
\hline Centrifuge & Rotor/Type & Manufacturer \\
\hline Tabletop centrifuge & 5424 & Eppendorf, Germany \\
\hline Refrigerated tabletop centrifuge & $5417 \mathrm{R}$ & Eppendorf, Germany \\
\hline RC6 Plus centrifuge & F9, F10, F21S FiberLite, F42 & Sorvall \\
\hline WX Ultra centrifuge & T647.5, T125.0 & Sorvall \\
\hline Discovery M120 & S45A, AT3 & Sorvall \\
\hline
\end{tabular}

\subsubsection{Bacterial strains}

Different strains of $E$. coli were used for cloning and protein expression purposes. NEB10beta (C3019, New England Biolabs) strain was used for cloning, whereas NEBexpress I ${ }^{\mathrm{q}}$ 
(C3037, New England Biolabs), TOP10F' (Invitrogen, Karlsruhe) and MDS ${ }^{\mathrm{TM}_{4}}$ (Scarab Genomics, LLC) strains were used for recombinant protein expression. TOP10F' was used for expression of Ran constructs, MDS1 for expression of Xpo6 constructs for optimized yield.

\subsubsection{Media for $E$. coli cultures}

Table 6-3 E. coli culture media

\begin{tabular}{|c|c|c|}
\hline LB medium & 2YT medium & TB medium \\
\hline $\begin{array}{l}\text { Used for DNA preparation } \\
\text { cultures }\end{array}$ & $\begin{array}{l}\text { Used for transformant recovery } \\
\text { protein expression pre/cultures }\end{array}$ & $\begin{array}{l}\text { Used for protein expression } \\
\text { cultures }\end{array}$ \\
\hline $\begin{array}{l}10 \text { g Tryptone } \\
5 \text { g Yeast extract } \\
10 \text { g NaCl } \\
\text { dd } \mathrm{H}_{2} \mathrm{O} \text { to } 1 \mathrm{~L} \\
\end{array}$ & $\begin{array}{l}16 \text { g Tryptone } \\
10 \mathrm{~g} \mathrm{Yeast} \text { extract } \\
5 \mathrm{~g} \mathrm{NaCl} \\
\mathrm{ddH} \mathrm{H}_{2} \mathrm{O} \text { to } 1 \mathrm{~L}\end{array}$ & $\begin{array}{l}12 \mathrm{~g} \text { Tryptone, } 24 \mathrm{~g} \text { Yeast extract } \\
10 \mathrm{~mL}(85 \%) \text { Glycerol } \\
2.31 \mathrm{~g} \mathrm{~K}_{2} \mathrm{HPO}_{4}, 16.4 \mathrm{~g} \mathrm{KH}_{2} \mathrm{PO}_{4} \\
\mathrm{ddH}_{2} \mathrm{O} \text { to } 1 \mathrm{~L}\end{array}$ \\
\hline Autoclaved & $\begin{array}{l}\text { Autoclaved, } 2 \% \text { glycerol and } 50 \mathrm{mM} \\
\mathrm{K}_{2} \mathrm{HPO}_{4} \text { is added for protein } \\
\text { expression cultures. } \\
0.5 \% \text { Glucose is added for } \\
\text { transformant recovery cultures. } \\
15 \mathrm{~g} \text { agar/L is added for agar plates. }\end{array}$ & Autoclaved \\
\hline
\end{tabular}

Antibiotic working concentrations: Ampicillin: $100 \mu \mathrm{g} / \mathrm{mL}$ Kanamycin: $\quad 50 \mu \mathrm{g} / \mathrm{mL}$

\subsubsection{Buffers and solutions}

Buffers and solutions for DNA work

50x TAE buffer

242 g Tris Base

$57.1 \mathrm{~mL}$ acetic acid $100 \mathrm{~mL} 0.5 \mathrm{M}$ EDTA pH 8.0 $\mathrm{ddH}_{2} \mathrm{O}$ to $1 \mathrm{~L}$

DNA ladder

$50 \mathrm{ng} / \mu \mathrm{L} 1 \mathrm{~kb}$-Ladder in orange sample buffer 10x T4 DNA Ligase Buffer

$500 \mathrm{mM}$ Tris $\mathrm{pH} 7.5$

$100 \mathrm{mM} \mathrm{MgCl} 2$

$100 \mathrm{mM}$ DTT

$10 \mathrm{mM}$ ATP

$250 \mu \mathrm{g} / \mathrm{mL}$ BSA

Orange G sample buffer

$10 \mathrm{mM}$ Tris- $\mathrm{HCl} \mathrm{pH} 8.0$

10 mM EDTA $\mathrm{pH} 8.0$

$50 \%(\mathrm{w} / \mathrm{v})$ glycerol

$25 \%$ (w/v) Orange G 10x PfuS buffer

$200 \mathrm{mM}$ Tris- $\mathrm{HCl}$

$200 \mathrm{mM} \mathrm{KCl}$

$15 \mathrm{mM} \mathrm{MgSO}_{4}$ $100 \mathrm{mM}\left(\mathrm{NH}_{4}\right)_{2} \mathrm{SO}_{4}$

$1 \%$ Tween-20

$1 \mathrm{mg} / \mathrm{mL}$ BSA

Buffers and solutions for protein work

\author{
10x SDS running buffer \\ 150 g Glycine \\ $30 \mathrm{~g}$ Tris Base \\ $12 \mathrm{~g} \mathrm{SDS}$ \\ $\mathrm{ddH}_{2} \mathrm{O}$ to $1 \mathrm{~L}$
}

Resuspension buffer 1 (RS1)

$500 \mathrm{mM} \mathrm{NaCl}$

$50 \mathrm{mM}$ Tris $\mathrm{pH} 7.5$

5 mM DTT

\author{
SDS sample buffer \\ 125 mM Tris pH 6.8 \\ $3 \%$ SDS \\ $50 \mathrm{mM}$ DTT \\ $1 \mathrm{M}$ Sucrose \\ bromophenol blue
}
Resuspension buffer 2 (RS2) $500 \mathrm{mM} \mathrm{NaCl}$
$50 \mathrm{mM}$ HEPES/KOH $\mathrm{pH} 8.2$
$2 \mathrm{mM} \mathrm{MgCl} 2$
$5 \mathrm{mM}$ DTT

Coomassie stock solution 2\% Coomassie Brilliant Blue $\mathrm{G} 250$ in $50 \%$ ethanol

Protein marker PageRuler Unstained protein ladder, Fermentas

G-actin buffer

$2 \mathrm{mM}$ Tris $\mathrm{pH} 8.0$

$0.2 \mathrm{mM} \mathrm{CaCl}_{2}$

$0.2 \mathrm{mM}$ ATP

$0.1 \mathrm{mM}$ DTT 


\subsubsection{Commercial screens for crystallization}

$\mathrm{AmSO}_{4}$, Classics, Classics II, Classics Lite, JSCG+, MPD, MBClass, MBClass II, ProComplex, PEGs, PEGs II, PACT, ComPAS screens were obtained from Qiagen (Hilden, Germany); MIDAS screen was obtained from Molecular Dimensions (Suffolk, UK); SaltRx and Index screens were obtained from Hampton Research (Aliso Viejo, CA, USA) and Wizard 1+2, Wizard 3+4 screens were obtained from Emerald Biosystems (Bainbridge island, WA, USA)

\subsubsection{Software}

Lasergene Suite 9.0 programs (DNASTAR, Inc. Madison, WI), Oligo 6.8 (Molecular Biology Insights, Cascade, CO, USA) and online tools IDT oligoanalyzer 3.1 (https://www.idtdna.com/analyzer/Applications/OligoAnalyzer/) were used for in silico cloning design and protein analysis. Gene designer 2.0.165 software (DNA2.0, Menlo Park, CA, USA) was used for design of gene synthesis constructs. Adobe Creative Suit 5 (Illustrator, Photoshop) were used for image preparation. Microsoft Office 2011 (for Mac) and Bookends 12.2 were used for writing and reference management. All crystal structure data was obtained from Protein Data Bank (PDB), visualization and analysis of the existing data and image preparation was performed with PyMOL (The PyMOL Molecular Graphics System, Version 1.5.0.4 Schrödinger, LLC.). For secondary structure predictions and sequence analyses, following online tools have been used: HHPred, PSI-BLAST and PSIPRED from bioinformatics toolkit of MPI for developmental biology (toolkit.tuebingen.mpg.de/), COBALT multiple alignment tool from NCBI (www.ncbi.nlm.nih.gov/tools/cobalt/) and Consensus secondary structure prediction tool (http://www.bioinf.manchester.ac.uk/dbbrowser/bioactivity/NPS2.html). Rock Maker software (Formulatrix, MA, USA) was used for the management of crystallographic work: design and pipetting of the crystallization screens, optimization of crystallization conditions, imaging and scoring of the crystallization drops.

\subsection{Methods}

\subsubsection{Standard methods in molecular biology}

All standard methods described here were performed as described by (Sambrook, 2001) 


\subsubsection{DNA purification from $E$. coli}

Plasmid DNA preparations from E. coli were done using NucleoSpin Plasmid kit (Macherey Nagel, Düren) at analytical scale (mini-prep), and using NucleoBond PC 100 (Macherey Nagel, Düren) at preparative scale (midi-prep) according to the manufacturer's instructions.

\subsubsection{Agarose gel electrophoresis}

DNA fragments were separated on agarose gels in 1x TAE running buffer. Agarose concentration of the gels varied between $1-1.5 \%$ in 1 xTAE depending on the size of the fragments to be separated. $0.05 \mu \mathrm{g} / \mathrm{mL}$ ethidium bromide was added to liquid agarose for visualization. Samples were mixed with at least 1/5 volume of Orange G loading buffer.

\subsubsection{DNA isolation from agarose gels}

DNA fragments from agarose gels were extracted using the Zymoclean Gel DNA recovery Kit (Zymo research, Freiburg) according to the manufacturer's instructions. DNA concentration was determined using Thermo NanoDrop 2000C spectrophotometer. (According to extinction at $260 \mathrm{~nm}\left(\mathrm{E}_{260}\right)$ and $\mathrm{E}_{260}=1$ corresponding to $50 \mu \mathrm{g} / \mathrm{mL}$ dsDNA)

\subsubsection{DNA restriction digestion \& ligation}

All restriction enzymes (RE) were purchased from New England Biolabs and the digestions were performed according to the manufacturer's instructions at $37^{\circ} \mathrm{C}$ for $1-2$ hours. Vector preparations were dephosphorylated following the restriction digestion using Fast Alkaline Phosphatase (Fermentas) for $30 \mathrm{mins}$ at $37^{\circ} \mathrm{C}$, in order to prevent reannealing of the vector and in order to reduce background during cloning. Vector (50ng) and insert fragments ( 3 fold molar excess to vector) were ligated using $100 \mathrm{ng} / \mu 1 \mathrm{~T} 4$ DNA ligase (produced by Steffen Frey, department of cellular logistics) and 1x Ligase buffer in a 10 $\mathrm{uL}$ reaction. For sticky-sticky ligations the reaction was incubated $30 \mathrm{mins}$ at $37^{\circ} \mathrm{C}$, for blunt ligations overnight at $16^{\circ} \mathrm{C}$. A control reaction without insert DNA was run in parallel as a negative control. $1 \mu \mathrm{L}$ of the ligation reaction was transformed into electrocompetent E.coli.

\subsubsection{Electroporation of E. coli}

Electrocompetent E. coli cells were prepared by Gabriele Kopp according to (Sambrook, 2001 ) and aliquoted in ready-to use amounts, which are then stored at $-80^{\circ} \mathrm{C}$. An aliquot of cells were slowly thawed and kept on ice. $45 \mathrm{uL}$ of electrocompetent E.coli cells were combined with $1 \mathrm{uL}$ of the ligation reaction (or with $<20 \mathrm{ng}$ plasmid DNA) in an 
electroporation cuvette (BioRad), which is placed on ice. Electroporation was performed using GenePulser (BioRad) according to the manufacturer's instructions. Cells were recovered in $1 \mathrm{~mL} 2 \mathrm{YT}+$ Glucose medium for $1 \mathrm{~h}$ at $37^{\circ} \mathrm{C}$ on a thermo shaker. The transformants are selected on 2YT-agar plates supplemented with corresponding antibiotics, overnight at $37^{\circ} \mathrm{C}$.

\subsubsection{Polymerase Chain Reaction (PCR)}

PCR was performed for the amplification of desired DNA fragments from DNA templates. Restriction sites for the subsequent cloning of the amplified DNA fragments were introduced to the 5' overhangs of the primers, if not already present in the template vector. PfuS triple mix (a mixture of DNA polymerase, pyrophosphatase and dUTPase, prepared by Steffen Frey, department of cellular logistics) was used as the thermostable DNA polymerase, instead of the common Taq polymerase. PfuS is a fusion of Pfu polymerase with the thermostable DNA-binding domain of Sac7d or Sso7d, which provided high speed $(\sim 3.3 \mathrm{~kb} / \mathrm{min})$ and high fidelity proofreading to the DNA polymerase. A typical PCR reaction ingredients and standard PCR conditions used in this study are shown below. For the annealing step, the temperature was adjusted to a lower temperature than the melting temperature of the primers. In most cases, $62^{\circ} \mathrm{C}$ was used as the standard. In problematic cases gradient PCR was performed. The annealing temperature was varied within the same block, to find the optimum conditions for the reaction.

Table 6-4 PCR reaction ingredients

\begin{tabular}{|ll|}
\hline Ingredient & Amount \\
\hline DNA & $50 \mathrm{ng}$ \\
\hline Forward primer $\mathbf{( 1 0 0} \boldsymbol{\mu M})$ & $1 \mu \mathrm{L}$ \\
\hline Reverse primer $\mathbf{( 1 0 0} \boldsymbol{\mu M})$ & $1 \mu \mathrm{L}$ \\
\hline $\mathbf{1 0 x}$ PfuS buffer & $10 \mu \mathrm{L}$ \\
\hline dNTP mix (2.5 mM each) & $10 \mu \mathrm{L}$ \\
\hline DMSO & $2 \mu \mathrm{L}$ \\
\hline PfuS triple mix & $1 \mu \mathrm{L}$ \\
\hline ddH & up to $100 \mu \mathrm{L}$ \\
\hline
\end{tabular}

Table 6-5 PCR conditions

\begin{tabular}{|l|l|l|l|} 
Step & Duration & $98.5^{\circ} \mathrm{C}$ & Repeat \\
\hline Initial denaturation & $2 \mathrm{~min}$ & $98.5^{\circ} \mathrm{C}$ & - \\
\hline Denaturation & $30 \mathrm{sec}$ & Depends on primer $\mathrm{Tm}$ & \multirow{2}{*}{$30-35$ cycles } \\
\hline Annealing & $30 \mathrm{sec}$ & $68^{\circ} \mathrm{C}$ & \\
\hline Elongation & $1 \mathrm{~min} / 2 \mathrm{~kb}$ product & $68^{\circ} \mathrm{C}$ & \\
\hline Final elongation & $10 \mathrm{~min}$ & \\
\hline
\end{tabular}




\subsubsection{Mutagenesis PCR}

Insertions, deletions or point mutations were introduced to a target vector by using mutagenesis PCR. Primers carried the nucleotides to be added to or mutated in the target vector at the 5' ends, for deletions primers were designed to anneal next to the region to be deleted. The mixture for mutagenesis PCR is essentially the same as the normal PCR. The PCR reaction was either purified directly with Zymogen kit, or isopropanol precipitation is performed. 2 volumes isopropanol was added to the PCR reaction at RT and precipitated in a tabletop centrifuge at $14 \mathrm{k}$ rpm for $15^{\prime}$. The pellet was washed in $70 \% \mathrm{EtOH}$, shortly pelleted again and air dried carefully until all EtOH is evaporated. Pellet was resuspended in $88 \mu \mathrm{L}$ of water, and combined with $10 \mu \mathrm{L}$ NEB buffer 4 and $1 \mu \mathrm{L}$ of DpnI $(20 \mathrm{U} / \mu \mathrm{L}$, NEB). The digestion reaction was incubated for $>2 \mathrm{~h}$ at $37^{\circ} \mathrm{C}$. DpnI specifically cleaves methylated and hemimethylated DNA strands, degrading the template DNA and leaving the PCR produced strands intact. DNA was separated on 1\% agarose gel and purified. 50 ng of the DNA was phosphorylated (if primers were not already 5 ' phosphorylated) and ligated as follows. $1 \mu \mathrm{L} \mathrm{10x} \mathrm{T4} \mathrm{ligase} \mathrm{buffer} \mathrm{and} 0.5 \mu \mathrm{L}$ PNK (Fermentas) were added to the PCR product, the volume was brought to $9.5 \mu \mathrm{L}$ with $\mathrm{ddH}_{2} \mathrm{O}$. The reaction was incubated for $30 \mathrm{~min}$ at $37^{\circ} \mathrm{C}$. $0.5 \mu \mathrm{L}$ T4 DNA Ligase was added and incubated for another 60 minutes. $1 \mu \mathrm{L}$ of this mixture was transformed to $45 \mu \mathrm{L}$ electrocompetent $E$. coli as described above.

\subsubsection{Gibson assembly reaction}

Gibson assembly reaction (GAR) (Gibson et al., 2009) was used for seamless cloning. It is especially useful in cases, where a suitable RE site can not be found, or when no additional amino acids are wanted within the construct resulting from a RE site between the tag and the desired protein. The insert template is created by PCR, with primers carrying the insertion sites to the vector at the 5' end flanks. The vector can be just linearized between the insertion sites, or ideally also created by PCR. The insert created now carries overlapping (identical) sequences to the vector ends. Gibson assembly utilizes three enzymatic activities for the insertion of the template to the vector, a 5'-3' T5 exonuclease, Phusion DNA polymerase and Taq DNA ligase, in a one step reaction (Gibson et al., 2009). Exonuclease creates 3' overhangs, which are compatible (by design) and can anneal. The Tm of the overlapping sequence is adjusted carefully for optimal annealing at the incubation temperature, that is $46^{\circ} \mathrm{C}$. After annealing, the gaps are filled by the polymerase, and nicks are annealed by the ligase. Equimolar amounts of vector and insert 
templates $(15$ fmol each, total $2 \mu \mathrm{L})$ is incubated with $2 \mu \mathrm{L}$ of the home-made Gibson assembly mix (prepared by Dirk Görlich, Department of Cellular Logistics) at $46^{\circ} \mathrm{C}$ for 30 mins. Then $0.1-0.5 \mu \mathrm{L}$ of this reaction is transformed to electrocompetent $E$. coli.

\subsubsection{Design and synthesis of oligos, DNA sequencing}

Primers were designed using Seqbuilder (Lasergene Suit) and Oligo 6.8 programs and online tools such as IDT oligoanalyzer 3.1. All oligos were ordered from Sigma-Aldrich DNA sequencing was performed by SeqLab (Göttingen) and results were analyzed by Seqman software (Lasergene Suite). The constructs for gene synthesis were designed using Gene designer 2.0.165 software for removal of possible RE sites and repetitive sequences from the constructs. All gene synthesis constructs were ordered from GenScript USA Inc. (NJ, USA).

\subsubsection{Bacterial expression vectors}

The table below lists the bacterial expression vectors created in this study and used in the experiments that are included in the thesis. The tag of the recombinant protein was either removed during purification, or left depending on the purpose of the further assays.

Table 6-6 Recombinant expression vectors used in this study

\begin{tabular}{|c|c|c|}
\hline Plasmid name & Expressed protein (with tags) & Purpose \\
\hline pKG031 & H14-zz-brSumo-hsRanQ69L(5-180) & Human RanGTP used in all experiments \\
\hline pKG050 & H14-zz-SumoStar-hsProfilin1 & $\begin{array}{l}\text { Tagged human profilin for profilactin / } \\
\text { export complex formation in HeLa lysate }\end{array}$ \\
\hline pKG059 & H14-zz-brSumo-hsProfilin1 & $\begin{array}{l}\text { Untagged human profilin used in complex } \\
\text { formations / binding assays }\end{array}$ \\
\hline pKG061 & H14-zz-SumoStar-hsXpo6 & Tagged human Xpo6 for binding assays \\
\hline pKG063 & H14-brSumo-xlXpo6 & $\begin{array}{l}\text { Xenopus Xpo6 for binding assays \& } \\
\text { alpaca immunizations }\end{array}$ \\
\hline pKG070 & H14-brSumo-hsXpo6 & $\begin{array}{l}\text { Untagged human Xpo6 for complex } \\
\text { formation and crystallization }\end{array}$ \\
\hline pKG091 & H14-brSumo-hsXpo6-Tr1 & Loop truncations for human Xpo6 \\
\hline pKG092 & H14-brSumo-hsXpo6-Tr2 & Loop truncations for human Xpo6 \\
\hline pKG093 & H14-brSumo-hsXpo6-Tr3 & Loop truncations for human Xpo6 \\
\hline pKG094 & H14-brSumo-hsXpo6-Tr4 & Loop truncations for human Xpo6 \\
\hline pKG095 & H14-brSumo-hsXpo6-Tr5 & Loop truncations for human Xpo6 \\
\hline pKG096 & H14-brSumo-hsXpo6-AL1 & Loop truncations for human Xpo6 \\
\hline pKG097 & H14-brSumo-hsXpo6-AL3 & Loop truncations for human Xpo6 \\
\hline pKG099 & H14-brSumo-hsXpo6-Tr6 & Loop truncations for human Xpo6 \\
\hline pKG108 & H14-brSumo-ggXpo6 & Chicken Xpo6 for binding assays \\
\hline pKG109 & H14-brSumo-dpXpo6 & D. purpureum Xpo6 for binding assays \\
\hline pKG111 & H14-brSumo-dfXpo6 & D. fasciculatum Xpo6 for binding assays \\
\hline
\end{tabular}

\subsubsection{SDS PAGE}

The analysis of the recombinant protein expression and purification as well as the binding assays, complex formation and gel filtration was performed with Sodiumdodecylsulfate 
polyacrylamide gel electrophoresis (SDS-PAGE) followed by visualization of the proteins by Coomassie staining. The gradient SDS polyacrylamide gels were prepared by Gabriele Kopp according to the Table 6-7. Gels were run for $1 \mathrm{~h}$ at $50 \mathrm{~mA}$ constant current in $1 \mathrm{x}$ SDS running buffer. Subsequently, proteins were fixed to the gel by heating the gel in 3\% acetic acid and stained with a 1:100 dilution of the Coomassie stock solution. Gels were destained in $\mathrm{H}_{2} \mathrm{O}$ and documented using an EPSON scanner.

Table 6-7 Gradient polyacrylamide gel solutions

\begin{tabular}{|l|l|l|l|}
\hline Ingredient & Heavy gel (16\%) & Light gel (7.5\%) & Stacking gel (4.5\%) \\
\hline app. for 10 gels & $200 \mathrm{~mL}$ & $200 \mathrm{~mL}$ & $100 \mathrm{~mL}$ \\
\hline 2 M Tris pH 8.8 & $40 \mathrm{~mL}$ & $40 \mathrm{~mL}$ & ---- \\
\hline $\mathbf{0 . 5}$ M Tris pH 6.8 & ---- & ---- & $15 \mathrm{~mL}$ \\
\hline $\mathbf{H}_{\mathbf{2}}$ O & $32 \mathrm{~mL}$ & $107 \mathrm{~mL}$ & $68 \mathrm{~mL}$ \\
\hline $\mathbf{2}$ M Sucrose & $10 \mathrm{~mL}$ & ---- & ---- \\
\hline $\mathbf{8 7 \%}$ Glycerol & $8 \mathrm{~mL}$ & ---- & ---- \\
\hline $\mathbf{1 0 \%}$ SDS & $2 \mathrm{~mL}$ & $2 \mathrm{~mL}$ & $2 \mathrm{~mL}$ \\
\hline Rotiphorese Gel 30 & $108 \mathrm{~mL}$ & $51 \mathrm{~mL}$ & $15 \mathrm{~mL}$ \\
\hline TEMED & $120 \mu \mathrm{L}$ & $120 \mu \mathrm{L}$ & $100 \mu \mathrm{L}$ \\
\hline APS (10\%) & $2 \times 580 \mu \mathrm{L}$ & $2 \times 580 \mu \mathrm{L}$ & $1 \mathrm{~mL}$ \\
\hline
\end{tabular}

\subsubsection{Recombinant protein expression and purification}

\subsubsection{Recombinant protein expression in E. coli}

All proteins except actin were expressed in E. coli strains, selected for the optimal yield (6.1.3). The optimal expression conditions were determined for each protein individually in order to maximize the yield. All proteins were purified natively. The common protocol for all recombinant expressions is described below. Variations are indicated for each construct. A pre-culture was inoculated in 2YT medium supplemented with appropriate antibiotics from a single colony, containing the desired construct. Pre-culture was grown overnight at $30-37^{\circ} \mathrm{C}$ with agitation. The next day, pre-cultures were used to inoculate the expression cultures in TB medium. The cultures were grown further at $30-37^{\circ} \mathrm{C}$ and then diluted with more $\mathrm{TB}$ medium to $\mathrm{OD} 600 \approx 2$, and brought to the expression temperature. The dilution step with pre-cooled medium enables a quick cooling of the cultures for cold expressions. For Ran and Xpo6 constructs expression was performed overnight at $18^{\circ} \mathrm{C}$, for profilin constructs $3-6 \mathrm{~h}$ at $25-30^{\circ} \mathrm{C}$. The cultures were grown at least $30 \mathrm{~min}$ at the expression temperature and expression was induced with varying concentrations of Isopropyl- $\beta$-D-thiogalactopyranosid (IPTG) $(100 \mu \mathrm{M}$ for Xpo6 and Ran, $200 \mu \mathrm{M}$ for profilin). Expression was carried out under constant agitation (85-100 rpm). After the expression the density of the culture is measured. Prior to harvesting $1 \mathrm{mM}$ 
phenylmethanesulfonylfluoride (PMSF) was added to the culture as protease inhibitor and the culture is poured to centrifuge tubes containing EDTA to a final concentration of 10mM. Cells were sedimented for 10 mins at $6000 \mathrm{rpm}$ using F9 rotor, Sorvall. Medium was removed and the cells were resuspended to $75-100 \mathrm{OD} / \mathrm{mL}$ concentration in their corresponding resuspension buffers (RS1 for Xpo6 and profilin constructs, RS2 for Ran constructs). The cells were flash frozen in liquid nitrogen. The resuspension can be stored at this stage for extended periods at $-80^{\circ} \mathrm{C}$, or immediately processed further.

\subsubsection{Native protein purification with $\mathrm{Ni}^{2+}$ affinity chromatography}

The cell resuspensions were thawed in warm water and placed on ice. The freeze thaw cycle also contributes to the lysis of the cells. The resuspension was sonified $3 \times 5 \mathrm{~min}$ at $40 \%$ duty cycle with output value of 10 (Branson Sonifier 450, CT, USA). Lysed cell suspension was ultracentrifuged at $37000 \mathrm{rpm}$ at $4^{\circ} \mathrm{C}$ for $1.5 \mathrm{~h}$ in $\mathrm{T} 647.5$ rotor. The supernatant, containing the soluble proteins, was used for affinity purification of the desired recombinant protein. Since all proteins expressed and used in this study have an Nterminal 14-His-Tag, $\mathrm{Ni}^{2+}$ affinity chromatography was performed to enrich the recombinant proteins. The Ni-EDTA matrix (prepared by Dirk Görlich) was equilibrated with the corresponding resuspension buffer. The lysate (supernatant after ultracentrifugation step) was added to the equilibrated matrix and 15-20 mM imidazole was added to repress non-specific binding of bacterial proteins to the matrix. At this imidazole concentration, 14 His tag can still efficiently bind to the matrix, whereas the contamination with bacterial proteins is significantly reduced. The binding to the matrix is done at least for $2 \mathrm{~h}$ in cold room with gentle rotation. Matrix to lysate ratio was optimized for individual proteins for maximum recovery. The mixture was applied to a chromatography column of appropriate size. The flow-through was collected. The now protein-bound resin was washed twice with resuspension buffer containing $25-30 \mathrm{mM}$ imidazole to remove low affinity contaminants. Elution of the protein was performed with resuspension buffer containing $0.5 \mathrm{M}$ imidazole. The collected fractions were analyzed by Amido Black quick staining and peak fractions were pooled together. The protein concentration was determined using NanoDrop. Eluted protein in this case still contained the N-terminal tag. A buffer exchange was performed to remove the imidazole and bring proteins to desired conditions using PD10 desalting columns (GE healthcare).

When the N-terminal tag was not needed any further, proteins were eluted by cleaving at the protease recognition site between the N-terminal affinity tag and the protein. In this 
case after washing step, the corresponding protease $(100 \mathrm{nM}$ for Sumo protease, $>600 \mathrm{nM}$ for SumoStar protease, both prepared by Steffen Frey) in resuspension buffer containing $5 \mathrm{mM}$ imidazole was quickly passed through the resin (by applying pressure) and cleavage was done $>1 \mathrm{~h}$ in cold room. The cleaved protein was collected by addition of more resuspension buffer containing $5 \mathrm{mM}$ imidazole. The collected fractions were analyzed by Amido Black quick staining and peak fractions were pooled together. The protein concentration was determined by measuring absorption at 280nm using NanoDrop, using the extinction coefficients calculated by Protean (Lasergene suit). The purity of the proteins was quite satisfactory with the described procedure. However, if additional purification was needed (for crystallization, or removal of protease or truncation products) protein was subjected to size exclusion chromatography (gel filtration). Purified proteins were aliquoted, supplemented with $250 \mathrm{mM}$ sucrose as cryoprotectant, flash-frozen in liquid nitrogen and stored at $-80^{\circ} \mathrm{C}$. Gel samples were taken at each step of expression/purification in order to follow the induction, solubility, stability and purity of the protein.

\subsection{Purification of Ran constructs}

Purification of Ran was a little more elaborate than the others, due to the bound nucleotide. In this study, a mutant (Q69L) and truncated version of Ran (5-180) was used. Q69L mutation prevents the GTPase activity of Ran (Klebe et al., 1995), hence the mutant Ran can not hydrolyze GTP. The C-terminal switch destabilizes the GTP-bound of Ran and weakens NTR interactions (Richards et al., 1995). In order to keep Ran in GTP bound form, special steps were implemented in its purification. After binding to Ni-matrix $30 \mu \mathrm{M}$ GTP was added in all steps, and an additional wash step with $2 \mathrm{mM}$ ATP was included, to remove bound bacterial chaperones. After the elution from Ni-matrix, protein was applied to SP-sepharose cation exchange chromatography and eluted with increasing salt. This way I could remove a major truncation that occurred during recombinant expression or the sumo protease used during cleavage. But also GTP-bound actin can well be separated on SP-sepharose than other nucleotide forms, such that one obtains a homogenous Ran sample in GTP bound form.

The nucleotide states of Ran constructs were controlled by the following protocol. $500 \mu \mathrm{L}$ of $15 \mu \mathrm{M}$ Ran was buffer exchanged to $50 \mathrm{mM}$ Tris $\mathrm{pH} 7.5$, denatured at $95^{\circ} \mathrm{C}$ for 5 mins. The solution was immediately diluted to $2 \mathrm{~mL}$ with $50 \mathrm{mM}$ Tris $\mathrm{pH}$ 7.5. Precipitants were removed by centrifugation at $14 \mathrm{k} \mathrm{rpm}$ in a cooled tabletop centrifuge. The supernatant was 
applied to a MonoQ HR 5/5 column equilibrated with $50 \mathrm{mM}$ Tris $\mathrm{pH} 7.5$, and the salt concentration was increased in a shallow gradient to $500 \mathrm{mM}$. The bound nucleotide (GTP/GDP) can be well separated with this protocol, and the results are compared to a GDP/GTP standard to determine the nucleotide state.

\subsubsection{Purification of skeletal muscle actin}

Actin was purified from chicken muscle acetone powder as described in (Spudich and Watt, 1971; Pardee and Spudich, 1982) with some minor modifications to the procedure. All steps were carried out in the cold room. All materials and buffers involved in the purification were placed into the cold room the night before use. $1000 \mathrm{~g}$ of very fresh, skinless, boneless chicken breast was purchased on the day of delivery, and brought to the lab in a cool box. All fat or remaining blood vessels were removed from the meat. The meat was cut into smaller pieces and finely ground using a meat grinder. All connecting tissues, that were building up during the grinding procedure were removed. The mince was extracted in five steps using sterile cheesecloth to separate extract and cell mass.

Extraction 1: The mince was stirred for 10 minutes in $1 \mathrm{~L}$ ice-cold extraction buffer I $(0.1$ $\mathrm{M} \mathrm{KCl}, 0.15 \mathrm{M}$ Potassium phosphate; $\mathrm{pH}$ 6.5). The extract was filtered using sterile cheesecloth.

Extraction 2: The mince was further stirred in $2 \mathrm{~L}$ ice-cold extraction buffer II $(0.05 \mathrm{M}$ $\mathrm{NaHCO}_{3}$ ) for 5 minutes. It is important not to exceed the extraction time in this buffer as it reduces the actin yield. The extract was filtered using sterile cheesecloth.

Extraction 3: The mince was stirred 10 minutes in $1 \mathrm{~L}$ ice cold extraction buffer III $(1 \mathrm{mM}$ EDTA, pH 7.0) and filtered using sterile cheesecloth.

Extraction 4: The mince was extracted twice in $1 \mathrm{~L}$ ice-cold water for 5 minutes by constant stirring and filtered using sterile cheesecloth.

Extraction 5 (repeated five times): The final five extractions were each in $1 \mathrm{~L}$ ice cold acetone for 10 minutes by carefully stirring with a glass rod. The extractions were done at room temperature in a fume hood but the acetone was kept ice cold at all times. Each extraction was filtered using sterile cheesecloth.

The final extract was spread in a large tray formed with aluminum foil. The tray was covered with clean cheesecloth, making sure that the cloth did not touch the mince. The 
mince was air dried in a hood over night. The dried acetone powder was filled into $50 \mathrm{ml}$ Falcon tubes and stored at $-80^{\circ} \mathrm{C}$. The acetone powder is stable for several months/years.

$11 \mathrm{~g}$ Acetone Powder was resuspended with $220 \mathrm{~mL}$ G-actin Buffer and stirred slowly at room temperature for 30 minutes. The liquid was collected and the remaining debris was resuspended with $150 \mathrm{~mL}$ more G-actin buffer for 5 more minutes. The liquid collected (approximately $300 \mathrm{~mL}$ ) was centrifuged at $18000 \mathrm{rpm} 4^{\circ} \mathrm{C}$ for $30 \mathrm{~min}$ in $\mathrm{F} 21$ rotor (Sorvall). The supernatant volume was measured and for $300 \mathrm{~mL}$ of supernatant, following ingredients were added while slowly stirring at room temperature:

$\begin{array}{ll}5 \mathrm{~mL} \text { of } 3 \mathrm{M} \mathrm{KCl} & \text { (50 mM final) } \\ 0.6 \mathrm{~mL} \text { of } 1 \mathrm{M} \mathrm{MgCl}_{2} & \text { (2 mM final }) \\ 3 \mathrm{~mL} \text { of } 0.1 \mathrm{M} \mathrm{ATP} & \text { (1 mM final })\end{array}$

Under these conditions, actin was left to polymerize for $1 \mathrm{~h}$ at room temperature, with slow stirring. After $1 \mathrm{~h}$ solution became thick and viscous indicating polymerization of G-actin into $\mathrm{F}$-actin. $\mathrm{KCl}$ concentration was increased to $0.6 \mathrm{M}$ by adding $12 \mathrm{~g}$ of $\mathrm{KCl}$ as powder while stirring. The solution was stirred for 20-30 more minutes at room temperature. The solution was centrifuged at $42000 \mathrm{rpm} 4^{\circ} \mathrm{C}$ for $1 \mathrm{~h}$ in $\mathrm{T} 1250$ rotor. The pellet containing the F-actin was resuspended with $\mathrm{G}$-actin buffer up to $15 \mathrm{~mL}$ volume. The resuspension was dialyzed against G-actin buffer for 48 hours in a dialysis bag with a cutoff value 6-8000 Da. The buffer was changed three times in 48 hours. The solution afterwards was centrifuged at $95000 \mathrm{rpm} 4{ }^{\circ} \mathrm{C}$ for $30 \mathrm{~min}$ in $\mathrm{S} 55 \mathrm{~A}$ rotor in order to get rid of precipitates and impurities.

G-actin concentration determination: $[\mathrm{G}-\operatorname{actin}(\mathrm{mg} / \mathrm{ml})]=\left(\mathrm{Abs}_{290} / 0.62\right) \times$ dilution factor

\subsubsection{HeLa cytoplasmic extract preparation}

HeLa cytoplasmic extracts were a courtesy of Dr. Berthold Kastner, Department of cellular biochemistry, MPI-BPC. The protocol is based on (Mayeda and Krainer, 1999), with some modifications. 4 liters of HeLa resuspension culture was pelleted and the pellet was washed 3x with 1x PBS (10x PBS: $\left.130 \mathrm{mM} \mathrm{NaCl}, 20 \mathrm{mM} \mathrm{KPO}_{4}\right) 10 \mathrm{~min}$ each. Pellet is weighed and weight (in g) was multiplied by 0.96 to get the packed cell volume in $\mathrm{ml}$. This value was then multiplied by 0.03 to get the cell number (in $10^{10}$ ). The cells were then resuspended in 1.25x volume MC buffer (10 mM HEPES KOH pH 7.6, $10 \mathrm{mM} \mathrm{KOAc,} 0.5$ $\mathrm{mM}$ MgOAc, $5 \mathrm{mM}$ DTT, 1x complete EDTA free proteinase inhibitor). The resuspension was incubated $5 \mathrm{~min}$ on ice and homogenized with 18 strokes using cell homogenizer. The 
homogenate was pelleted in Corex tubes at $13000 \mathrm{RCF}$ in SS34 rotor for $5 \mathrm{~min}$. The supernatant was the S10 cytoplasmic extracts used in this study. The S10 extract was aliquoted and frozen in liquid nitrogen. The extract is stable at $-80^{\circ} \mathrm{C}$. The $\mathrm{S} 10$ extract was further cleared at $90000 \mathrm{xg}$ before use in the binding experiments.

\subsubsection{DNasel coupling to cyanogen bromide activated sepharose}

In order to test the accessibility of actin's D-loop in the export complex, DNaseI matrix was prepared by coupling DNaseI from bovine pancreas (Sigma, D-5025) to cyanogen bromide-activated-Sepharose ${ }^{\circledR}$ 4B (Sigma, C9142). $500 \mathrm{mg}$ matrix was washed with $1 \mathrm{mM}$ $\mathrm{HCl}$ and let to swell for $30 \mathrm{~min}$ at room temperature. After swelling, the matrix was washed with $100 \mathrm{~mL}$ of $1 \mathrm{mM} \mathrm{HCl}, 10 \mathrm{~mL}$ water and with $2 \mathrm{~mL}$ coupling buffer (CB: $0.1 \mathrm{M}$ $\mathrm{Na} \mathrm{HCO}_{3} 50 \mathrm{mM} \mathrm{NaCl} \mathrm{pH} 8.3$ ) immediately before the coupling reaction. In the meantime, $5 \mathrm{mg}$ DNaseI was dissolved in $3 \mathrm{~mL}$ CB $(1.6 \mathrm{mg} / \mathrm{mL})$ and was incubated with $100 \mathrm{uL}$ Aprotinin matrix $(16 \mathrm{mg} / \mathrm{mL})$ for $15 \mathrm{~min}$ to remove the trypsin from the sample. The matrix was removed by centrifugation at $500 \mathrm{rpm}$ for $1 \mathrm{~min}$ on a tabletop centrifuge. Optionally, DNaseI can be incubated with protease inhibitors like PMSF for another 30-60 min. The concentration of the protein was measured with absorbance at $280 \mathrm{~nm}$. The $\mathrm{NaCl}$ concentration was increased to $500 \mathrm{mM}$. The washed matrix was split into two. One part was incubated with DNaseI and the other part with $\mathrm{CB}+500 \mathrm{mM} \mathrm{NaCl}$ over night in cold room. Next day, the flow through was taken and the protein concentration was measured. Absorbance zero at $280 \mathrm{~nm}$, means that all DNaseI was coupled to the sepharose matrix. Both matrices were washed with $\mathrm{CB}$ and quenched with $1 \mathrm{M}$ Tris $\mathrm{pH} 8.0$ in $1: 1 \mathrm{CB}$ for $2 \mathrm{~h}$ at room temperature. The matrices were extensively washed with $\mathrm{CB}$ and with $0.1 \mathrm{M}$ Acetate buffer $\mathrm{pH} 4.0,0.5 \mathrm{M} \mathrm{NaCl}$. The wash was repeated 4-5 times. The matrix was stored in $1 \mathrm{M} \mathrm{NaCl}$ at $4^{\circ} \mathrm{C}$.

\subsubsection{Binding assays for complex formation}

Binding assays were performed with purified components to test various conditions for their effect on complex formation. HeLa S10 extract was used as a source of cytoplasmic actin. Buffer conditions and additives varied depending on the purpose of the experiment, but the following conditions were kept constant. Binding assays were carried out in small mobicol columns plugged with $35 \mu \mathrm{m}$ pore size filters (MoBiTech, Göttingen). For most of the assays shown in this work, profilin was used as the tagged component, where all the other members of the complex were assembled. $50 \mu \mathrm{L}$ of undiluted cleared S10 extract and 250 pmol profilin was incubated in the presence of $5 \mathrm{mM}$ EDTA for $1 \mathrm{~h}$ in cold room. This 
was then diluted to $500 \mu \mathrm{L}$ with binding buffer with the addition of $20 \mu \mathrm{L}$ affinity matrix. The complex was immobilized on Ni-matrix via the N-terminal 14-His of the tagged component for $1-2 \mathrm{~h}$ in cold room with gentle rotation. The flow through (FT) was taken and the matrix was washed with $2 \times 200 \mu \mathrm{L}$ binding buffer. Then again in $500 \mu \mathrm{L}$ binding buffer, Xpo6 $(1 \mu \mathrm{M})$ and RanGTP $(1.2 \mu \mathrm{M})$ were added to the matrix and incubated for $3 \mathrm{~h}$ in cold room with gentle rotation. Xpo6 and Ran were pre-mixed for 1-2h without dilution, prior to addition to the matrix. FT was taken and the matrix was washed with $2 \times 200 \mu \mathrm{L}$ binding buffer. The complex was then eluted by cleavage of the tag with protease. SumoStar tagged components and respectively SumoStar protease were used in the binding assays, because the SumoStar substrate could not be cleaved by the eukaryotic proteases present in the S10 extract.

Another set of binding assays were performed on phenyl sepharose (PS) matrix (Phenyl Sepharose $^{\mathrm{TM}} 6$ Fast Flow (low subst.), Amersham Bioscience), which binds to nuclear transport receptors. In this case, all components were untagged. S10 extract and profilin were pre-incubated for 1h, which was followed by the addition of Xpo6 and RanGTP and $10 \mu \mathrm{L}$ PS matrix. FT was taken and the matrix was washed with $2 \times 200 \mu \mathrm{L}$ binding buffer. Xpo6 and all the other proteins that were bound to Xpo6 were retrieved from the matrix by SDS elution. The control experiments were performed without Xpo6, to ensure that the binding of the other components to PS matrix is specifically via Xpo6.

\subsubsection{Actin export complex formation for crystallization}

The export complex was formed in a two-step procedure in large scale for crystallization. $50 \mathrm{~mL}$ of undiluted, ultracentrifugation cleared S10 HeLa cytoplasmic extract was combined with $600 \mu \mathrm{L}$ of $500 \mu \mathrm{M}$ tagged profilin in the presence of $5 \mathrm{mM}$ EDTA in cold room with gentle rotation for $1 \mathrm{~h}$. This was diluted to $250 \mathrm{~mL}$ with binding buffer $(10 \mathrm{mM}$ Tris $\mathrm{pH}$ 7.5, $20 \mathrm{mM} \mathrm{NaAc}$ (sodium acetate, $\mathrm{CH}_{3} \mathrm{COONa}$, $\mathrm{pH}$ adjusted to 7.5), $1 \mathrm{mM}$ DTT) and $6 \mathrm{~mL} \mathrm{Ni-matrix} \mathrm{(equilibrated} \mathrm{with} \mathrm{binding} \mathrm{buffer)} \mathrm{was} \mathrm{added} \mathrm{in} \mathrm{the} \mathrm{presence} \mathrm{of} 10$ $\mathrm{mM}$ imidazole, to reduced unspecific binding from S10 extract to Ni-matrix. This was incubated in cold room for $2-2.5 \mathrm{~h}$ with gentle rotation. FT was taken and the matrix was washed with $10 \mathrm{~mL}$ binding buffer with $10 \mathrm{mM}$ imidazole and $10 \mathrm{~mL}$ of complex buffer (10 mM Tris $\mathrm{pH}$ 7.5, $20 \mathrm{mM} \mathrm{NaAc,} 2 \mathrm{mM} \mathrm{MgAc}$ (magnesium acetate, $\mathrm{Mg}\left(\mathrm{C}_{2} \mathrm{H}_{3} \mathrm{O}_{2}\right)_{2}$ ), 1 $m M D T T$ ) with $5 \mathrm{mM}$ imidazole. $7 \mathrm{~mL}$ of $56 \mu \mathrm{M}$ Xpo6 and $4.5 \mathrm{~mL}$ of $93 \mu \mathrm{M}$ RanQ69L(5180) were mixed and incubated $2 \mathrm{~h}$ in cold room without dilution. This mixture was buffer exchanged to complex buffer using PD10 desalting columns (GE healthcare). It was added 
to the Ni-matrix in $30 \mathrm{~mL}$ total volume in $5 \mathrm{mM}$ imidazole. Binding was performed $>3 \mathrm{~h}$, preferentially overnight. The longer incubation times increased the final yield of export complex. FT was taken, the matrix was washed with $10 \mathrm{~mL}$ complex buffer with $5 \mathrm{mM}$ imidazole. The tag of profilin was cleaved with $200 \mathrm{nM}$ SumoStar protease in $6 \mathrm{~mL}$ complex buffer $+5 \mathrm{mM}$ imidazole. The complex was eluted with complex buffer $+5 \mathrm{mM}$ imidazole. Fractions were analyzed by amido black quick staining, pooled and concentrated. The sample was ultra centrifuged $(90000 \mathrm{x}$ g) to remove aggregates and was applied to a Superdex ${ }^{\mathrm{TM}} 200$ 16/60 gel filtration column (GE healthcare) equilibrated with complex buffer. The gel filtration fractions were analyzed, pooled and concentrated to $\sim 15 \mathrm{mg} / \mathrm{mL}$ for crystallization purposes. Sample that was not immediately used for crystallization was aliquoted and snap-frozen in liquid nitrogen and stored at $-80^{\circ} \mathrm{C}$. This procedure yielded approximately $30 \mathrm{mg}$ of high purity actin export complex.

For Xpo6 6 RanGTP complex crystallization, purified and untagged proteins were mixed in a 1:1.2 ratio (Xpo6:RanGTP), incubated $2-3 \mathrm{~h}$ at $4^{\circ} \mathrm{C}$, buffer exchanged into $10 \mathrm{mM}$ Tris pH 7.5, $20 \mathrm{mM} \mathrm{NaAc}$ 20, $2 \mathrm{mM} \mathrm{MgAc} 2,1 \mathrm{mM}$ DTT and applied to Superdex 200 16/60 size exclusion column. The sample was concentrated to $15 \mathrm{mg} / \mathrm{mL}$.

For Xpo6 crystallization, affinity purified Xpo6 was concentrated, buffer exchanged to 10 $\mathrm{mM}$ Tris 7.5, $50 \mathrm{mM} \mathrm{NaCl}, 1 \mathrm{mM}$ DTT, applied to Superdex 200 16/60 size exclusion column. The sample was concentrated to $17 \mathrm{mg} / \mathrm{mL}$.

\subsubsection{Thermal stability analysis with Thermofluor}

Thermofluor experiments were performed to test the thermal stability of Xpo6, Xpo6•RanGTP and the actin export complex. Each sample was diluted to $5 \mu \mathrm{M}$ in $20 \mu \mathrm{L}$ of complex buffer (10 mM Tris pH 7.5, $20 \mathrm{mM} \mathrm{NaAc,} 2 \mathrm{mM} \mathrm{MgAc}$, 1mM DTT) containing 1x Sypro-Orange (Life Technologies). Three replicates of each sample $(20 \mu \mathrm{L})$ were pipetted in a Hard-Shell ${ }^{\circledR}$ 96-well plate (Bio-Rad). The plate was sealed with transparent MicroSeal ${ }^{\circledR}$ 'B' Seal (Bio-Rad) and briefly centrifuged to remove air bubbles from the wells. For the assay, CFX96 Real-Time System (C1000 Thermal Cycler, BioRad) was used. The temperature was gradually increased (with $1^{\circ} \mathrm{C}$ increments per cycle) from $30^{\circ} \mathrm{C}$ up to $95^{\circ} \mathrm{C}$. The Sypro-Orange fluorescence was measured after each temperature increment using the HEX channel. The raw data of the measurements were exported as excel sheets for further analysis. Graphical representations were prepared using Microsoft Excel 2011 for Mac. 


\subsubsection{Molecular weight analysis with static light scattering}

To determine the molecular weight of the complex, multi angle light scattering (MALS) was employed. The technique is based on two main principles: First, the amount of light that is scattered by a particle (protein, in this case) is directly proportional to the product of the molar mass and the molecular concentration. Second, the variation of scattered light with the scattering angle is proportional to the average size (radius) of scattering molecules (for particles with diameter $>\lambda / 20$ ). In the system we employ (miniDAWN TREOS), a high intensity polarized light $(659 \mathrm{~nm})$ is shot onto sample and the scattered light is detected at three different angles. This information together with measurements of concentration (UV and refractive index measurements) is used for the calculation of the molecular weight of the sample. The physical equations are not described here. The device is coupled to a gel filtration system, such that the sample is size separated before being analyzed. Calculations and analysis of results were performed by Astra ${ }^{\circledR} 6$ software (Wyatt Technology Europe).

\subsubsection{Polydispersity analysis by dynamic light scattering}

Dynamic light scattering is used to determine the size distribution profile of a given particle solution. Particles scatter light, and the intensity of the scattered light at a given angle fluctuates over time, because the particles undergo Brownian motion. The rate at which particles diffuse in a solution is related to their size (hydrodynamic radius $R_{h}$ ), when all other parameters are constant. DLS measures the fluctuations in light scattered by the sample over time in order to determine the size distribution of the particles in the sample. Thus, DLS can be used as a measure of sample heterogeneity (dispersity). A non-uniform (polydisperse) collection of particles is not ideal for crystallization; rather a homogenous (monodisperse) sample is desired. We used DynaPro NanoStar (Wyatt Technology Europe) for DLS measurements of the crystallization samples as a quality control. $10 \mu \mathrm{L}$ of sample is used per measurement with 20 acquisitions of 5 second each. DLS can also be used to analyze the size distribution of a given sample over a temperature range. This method was applied as an alternative to thermofluor assays to determine the thermostability of crystallization substrates.

\subsubsection{Limited proteolysis of crystallization substrates}

In order to identify flexible regions in the crystallization substrates, limited proteolysis was applied. $3 \mu \mathrm{g}$ protein was incubated with a dilution series of proteases (Trypsin Chymotrypsin, Promega, Germany / GluC, Roche, Germany) for $1 \mathrm{~h}$ at $22^{\circ} \mathrm{C}$. The 
concentration of the protease varied between $10 \mathrm{ng} / \mu \mathrm{L}$ and $8 \mathrm{pg} / \mu \mathrm{L} .15 \mu \mathrm{L}$ of SDS sample buffer containing $5 \mathrm{mM}$ EDTA and 5mM PMSF was added and the reaction was incubated at $95^{\circ} \mathrm{C}$ for 5 minutes to stop the reaction. $10 \mathrm{uL}$ of this sample was analyzed with SDSPAGE and bands of interest were cut and analyzed by mass spectrometry.

\subsubsection{Mass spectrometry}

The MS-MS analysis of the fragments created by limited proteolysis was done by Mass spectrometry in Henning Urlaub Lab, MPI-BPC. A more detailed analysis of the identified peptides and the identification of the protease cut sites was kindly done by Samir Karaca, using Maxquant software.

\subsubsection{Protein crystallization}

Proteins and protein complexes were purified to highest possible degree with affinity and size exclusion chromatography techniques and brought to high concentrations for crystallization. We used an automated system for large scale screening of crystallization conditions at the crystallization facility of MPI-BPC, run by Dr. Vlad Pena. As a general approach, vapor-diffusion method was used for crystallization. In this method, a small volume of the concentrated protein sample is mixed with the same volume of crystallization solution (reservoir, mother liquor), which consists of buffer, salt or precipitant; generally a combination of all three. The drop is incubated with the reservoir in an air tight sealed chamber, but with no physical contact to the reservoir. Two types of vapor diffusion techniques are used: the drop is either placed on a coverslip which seals the chamber from the top (hanging drop), or on a well above the reservoir level in the chamber, which is again tightly sealed (sitting drop). The difference in the concentration of components (mainly precipitant) between the drop and reservoir solution causes vapor diffusion of the solvent until equilibrium is reached. Shrinking of the drop due to water loss, leads to super saturation of the protein, which favors crystallization, under optimal conditions. With the robotic system, sitting drop vapor diffusion method was utilized in 96-well MRC plates (Molecular Dimensions, UK). In manual crystallization experiments, we employed hanging drop method.

\subsubsection{Sparse matrix screening}

There are a number of commercially available crystallization screens based on previous successful crystallization conditions and their derivations (sparse matrix screens). This is today the most commonly employed approach for initial crystallization trials. A broad 
spectrum of conditions (randomly) screened for hits. Crystallization drops were set up in 96 well sitting drop MRC plates (Molecular dimensions) using Cartesian Microsys Nano dispenser robot. $60 \mathrm{nl}$ of reservoir was pipetted followed by $60 \mathrm{nl}$ of protein $(15-17 \mathrm{mg} / \mathrm{ml})$ into the round wells of MRC plates. After pipetting, the plates were sealed tightly with a clear sealing tape and stored at $20^{\circ} \mathrm{C}$ or $4^{\circ} \mathrm{C}$ (both temperatures were screened for each condition) in automated robotic imager (Formulatrix). Crystal growth was monitored over time using Rock Maker software (Formulatrix), which was used to take images of the crystallization drops at regular intervals.

\subsubsection{Grid screening}

When an initial crystallization hit was obtained from sparse matrix screens, the corresponding condition was further optimized for increased crystal quality and size by screening around the initial crystallization conditions (grid screening). Grid screens were designed using RockMaker software (Formulatrix) by varying the concentration and $\mathrm{pH}$ of components in the initial hit condition around their original concentration and $\mathrm{pH}$. The plates were pipetted as described for sparse matrix screens and monitored on regular intervals.

\subsubsection{Manual drops}

For crystallization experiments such as in-situ proteolysis, seeding, or just to obtain larger crystals manual drops were set in microliter scale. Hanging drop method was employed, using 24-well EasyXtal tool (QIAGEN) plates with grease-free screw lids. $400 \mu \mathrm{L}$ of reservoir was placed in the well; $1-2 \mu \mathrm{L}$ of reservoir was mixed with $1-2 \mu \mathrm{L}$ of protein solution on the cover slip/lid. The lid is tightly screwed, and the plate is incubated in cold room / at room temperature. The dropswere monitored at regular time intervals by visual examination under Leica MZ6 microscope (Leica Microsystems).

\subsubsection{Seeding}

Seeding is a crystallographic technique where imperfect crystals are used to grow better diffracting, bigger crystals. The logic is to overcome the rate limiting nucleation step of crystallization. It is especially important, when the identified first hits can not easily be reproduced. The conditions required for nucleation and crystal growth might not always be identical. We used microseeding, to reproduce or improve crystals. The initial crystals were taken to a loop and washed in a fresh reservoir solution, and eventually left in the reservoir condition, where they grew. In $20 \mu \mathrm{L}$ reservoir in an eppendorf tube, the crystals 
were crushed by vigorous vortexing and subsequent sonication in a waterbath. This seed stock is then further diluted and the dilution series are mixed 1:1 with new protein sample for crystallization.

\subsubsection{Cryoprotection, crystal fishing and data collection}

When a crystallization drop was to be harvested, the solution was slowly exchanged to a "cryo-condition" without disturbing the crystals. This prevented ice formation inside or surrounding the protein crystal during freezing The cryo-protectant solution had the identical buffer and salt conditions as the reservoir, the precipitant (in this work, mostly PEG) concentration was increased to $25 \%$, and another suitable cryo-protectant (glycerol, ethylene glycol, xylitol, sucrose, PEG 400) was added if precipitant increase wasn't enough. After the drop solution was replaced with the cryo-colution, crystals were carefully fished with Nylon-loops mounted on a magnetic head (mounted CryoLoops, Hampton Research) of $0.06-0.5 \mu \mathrm{m}$ in size. Fished crystal was immediately in liquid nitrogen for freezing. Diffraction data from cryo-cooled crystals were collected at $100 \mathrm{~K}$ on a Pilatus detector at the Swiss Light Source (Switzerland) beamline PXII. 


\section{Abbreviations}

aa

ABP

ADP

ATP

ATPase

CAS

CRM1

C-terminus

DMSO

DNA

DTT

E. coli

EDTA

Exp/Xpo

F-actin

FG

G-actin

GDP

GFP

GTP

h

HEPES

His-tag

Imp

IPTG

$\mathrm{kDa}$

LB

MALS

MDa

$\min$

$\mathrm{N}$-terminus

$\mathrm{NE}$

NES

NLS

nmol
Amino acid

Actin binding protein

Adenosine 5'-diphosphate

Adenosine 5'-triphosphate

ATP hydrolase

cellular apoptosis susceptibility (a.k.a. Exportin 2)

Chromosomal region maintanence-1 (a.k.a. Exportin 1)

Carboxy-terminus

Dimethyl sulfoxide

Deoxyribonucleic acid

Dithiothreitol

Escherichia coli

Ethylene diamine tetraacetic acid

Exportin

Filamentous actin

Phenylalanine/Glycine

Globular actin

Guanosine 5'-diphosphate

Green fluorescent protein

Guanosine 5'-triphosphate

hours

4-(2-hydroxyethyl)-1-piperazineethanesulfonic acid

(in this study) amino terminal tag of 14 Histidine residues

Importin

Isopropyl- $\beta$-D-thiogalactoside

kilo Dalton

Luria Bertani medium

Multi angle light scattering

mega Dalton

minutes

Amino-terminus

Nuclear envelope

nuclear export signal

nuclear localization signal

nanomole 


$\begin{array}{ll}\text { Nup } & \text { Nucleoporin } \\ \text { NPC } & \text { nuclear pore complex } \\ \text { PEG } & \text { polyethylene glycol } \\ \text { PIP }_{2} & \text { Phosphatidylinositol 4,5-bisphosphate } \\ \text { PS } & \text { Phenyl sepharose } \\ \text { RanGTP } & \text { (GTP-bound)Ras related nuclear antigen } \\ \text { rpm } & \text { rotation per minute } \\ \text { SDS-PAGE } & \text { Sodium dodecyl sulfate gel electrophoresis } \\ \text { Sumo } & \text { Small ubiquitin like modifier } \\ \text { Tris } & \text { Tris(hydroxymethyl)aminomethane } \\ \text { Xpo6 } & \text { Exportin 6 } \\ \text { w/v } & \text { weight per volume } \\ \mu \mathrm{m} & \text { micrometer } \\ \mu \mathrm{M} & \text { micromolar }\end{array}$




\section{Acknowledgements}

In the first place, I thank my supervisor Dirk Görlich for trusting me with this challenging and exciting project, for his input and help throughout my $\mathrm{PhD}$. I still haven't reached the borders of your scientific knowledge, despite all my various questions all these years. I don't think I will ever meet another person as passionate about science as you.

I thank my thesis committee members Jörg Großhans and Reinhard Jahn for the valuable comments, criticism and discussions. I also express my sincere gratitude to my mentor Prof. Thomas Pieler, for the valuable advice and insights.

Crystallization was a big part of this study, and it would have been impossible without the excellent technical support of our crystallization facility; Jürgen Wawrzinek and Ulrich Steuerwald. I sincerely thank Uli for his help, ideas and suggestions in protein crystallization, sharing my enthusiasm, even fishing my first crystals. I thank Sergei Trakhanov for his help in X-ray data collection and the indexing of diffraction data from Xpo6 crystals.

I would like to thank Dirk Görlich, Steffen Frey and Koray Kırlı for the critical reading of the various parts of this thesis, Steffen Frey for being the first address in the case of "unsolvable" questions, and always bringing a new and better perspective.

This project was generously funded through GGNB Excellence Stipend and Boehringer Ingelheim Fonds $\mathrm{PhD}$ fellowship. I want to especially thank the BIF team for their support and the nice time we had in Cold Spring Harbor.

Sincere thanks go to,

Jürgen and Renate for the help with chromatography and clonings, and for all the pleasant conversations we had.

Gabi and Uwe for taking care of us in every possible way. It is priceless to have your gels, agar plates, culture media, competent cells, DNA preparations, chemicals and stock solutions ready to use whenever you need.

Steffen Burkhardt and Kerstin Grüniger in the name of the MolBio family, for their support and guidance throughout the MSc and after; for making the past 5 years in Göttingen more than just a PhD. 
Connie, for being such a helping hand when it came to the German bureaucracy and paperwork, for finding the easiest way out!

Hema Chug for the friendly motivational support, when crystallization seemed like a dead end street.

Heinz-Jürgen for all the excellent doctor-hats so far (in advance, also for my hat to come), for his excellent cakes and lovely conversations we had.

All members of the lab; for the discussions during seminars, for making the lab a hospitable place!

I especially thank Koray Kırlı, Metin Aksu and Samir Karaca, for their friendship, scientific discussions and motivation, for all the fun we have, for not letting me forget my mother language in a foreign country.

My heartfelt thanks go to my parents and family for the long-distance motivational support, and for their unconditional love. I love you all.

Finally, I would like to thank Maximilian; for holding my hand through the roughest times as well as in joy and happiness. I am so lucky to have you in my life.

Kevser 


\section{References}

Alberts B, Johnson A, Lewis J, Raff M, Roberts K, Walter P. (2002) Molecular Biology of the Cell, Fourth Edition. Garland Science,

Alberts B, Johnson A, Lewis J, Raff M, Roberts K, Walter P. (2007) Molecular Biology of the Cell. Garland Science,

Allen TD, Rutherford SA, Murray S, Sanderson HS, Gardiner F, Kiseleva E, Goldberg MW, Drummond SP (2007) A protocol for isolating Xenopus oocyte nuclear envelope for visualization and characterization by scanning electron microscopy (SEM) or transmission electron microscopy (TEM). Nat Protoc, 2: 1166-1172

Allingham JS, Klenchin VA, Rayment I (2006) Actin-targeting natural products: structures, properties and mechanisms of action. Cell Mol Life Sci, 63: 2119-2134

Andrade MA, Bork P (1995) HEAT repeats in the Huntington's disease protein. Nat Genet, 11: 115-116

Arai A, Mitsuhashi S, Saito Y, Komaki H, Sakuma H, Nakagawa E, Sugai K, Sasaki M, Robertson SP, Nishimura G, Yamamoto T, Nonaka I, Nishino I (2009) Nemaline (actin) myopathy with myofibrillar dysgenesis and abnormal ossification. Neuromuscul Disord, 19: 485-488

Bayliss R, Littlewood T, Stewart M (2000) Structural basis for the interaction between FxFG nucleoporin repeats and importin-beta in nuclear trafficking. Cell, 102: 99-108

Bednenko J, Cingolani G, Gerace L (2003) Importin beta contains a COOH-terminal nucleoporin binding region important for nuclear transport. J Cell Biol, 162: 391-401

Birbach A (2008) Profilin, a multi-modal regulator of neuronal plasticity. Bioessays, 30: 994-1002

Bischoff FR, Gorlich D (1997) RanBP1 is crucial for the release of RanGTP from importin beta-related nuclear transport factors. FEBS Lett, 419: 249-254

Bischoff FR, Klebe C, Kretschmer J, Wittinghofer A, Ponstingl H (1994) RanGAP1 induces GTPase activity of nuclear Ras-related Ran. Proc Natl Acad Sci U S A, 91: 2587-2591

Bischoff FR, Ponstingl H (1991a) Mitotic regulator protein RCC1 is complexed with a nuclear ras-related polypeptide. Proc Natl Acad Sci U S A, 88: 10830-10834

Bischoff FR, Ponstingl H (1991b) Catalysis of guanine nucleotide exchange on Ran by the mitotic regulator RCC1. Nature, 354: 80-82

Bohnsack MT, Czaplinski K, Gorlich D (2004) Exportin 5 is a RanGTP-dependent dsRNA-binding protein that mediates nuclear export of pre-miRNAs. $R N A, \mathbf{1 0}: 185-191$

Bohnsack MT, Regener K, Schwappach B, Saffrich R, Paraskeva E, Hartmann E, Gorlich D (2002) Exp5 exports eEF1A via tRNA from nuclei and synergizes with other transport pathways to confine translation to the cytoplasm. EMBO J, 21: 6205-6215

Bohnsack MT, Stuven T, Kuhn C, Cordes VC, Gorlich D (2006) A selective block of nuclear actin export stabilizes the giant nuclei of Xenopus oocytes. Nat Cell Biol, 8: 257-263

Boivin S, Kozak S, Meijers R (2013) Optimization of protein purification and characterization using Thermofluor screens. Protein Expr Purif, 91: 192-206

Bono F, Cook AG, Grunwald M, Ebert J, Conti E (2010) Nuclear import mechanism of the EJC component Mago-Y14 revealed by structural studies of importin 13. Mol Cell, 37: 211-222

Bork P, Sander C, Valencia A (1992) An ATPase domain common to prokaryotic cell cycle proteins, sugar kinases, actin, and hsp70 heat shock proteins. Proc Natl Acad Sci U S A, 89: 7290-7294

Brownawell AM, Macara IG (2002) Exportin-5, a novel karyopherin, mediates nuclear export of doublestranded RNA binding proteins. J Cell Biol, 156: 53-64 
Cansizoglu AE, Chook YM (2007) Conformational heterogeneity of karyopherin beta2 is segmental. Structure, 15: 1431-1441

Carlier MF (1990) Actin polymerization and ATP hydrolysis. Adv Biophys, 26: 51-73

Carlsson L, Nystrom LE, Lindberg U, Kannan KK, Cid-Dresdner H, Lovgren S (1976) Crystallization of a non-muscle actin. $J$ Mol Biol, 105: 353-366

Carlsson L, Nystrom LE, Sundkvist I, Markey F, Lindberg U (1977) Actin polymerizability is influenced by profilin, a low molecular weight protein in non-muscle cells. J Mol Biol, 115: 465-483

Chereau D, Kerff F, Graceffa P, Grabarek Z, Langsetmo K, Dominguez R (2005) Actin-bound structures of Wiskott-Aldrich syndrome protein (WASP)-homology domain 2 and the implications for filament assembly. Proc Natl Acad Sci U S A, 102: 16644-16649

Choi S, Yamashita E, Yasuhara N, Song J, Son SY, Won YH, Hong HR, Shin YS, Sekimoto T, Park IY, Yoneda Y, Lee SJ (2014) Structural basis for the selective nuclear import of the $\mathrm{C} 2 \mathrm{H} 2$ zinc-finger protein Snail by importin beta. Acta Crystallogr D Biol Crystallogr, 70: 1050-1060

Chou PC, Liang WC, Nonaka I, Mitsuhashi S, Nishino I, Jong YJ (2013) Intranuclear rods myopathy with autonomic dysfunction. Brain Dev, 35: 686-689

Clark TG, Merriam RW (1977) Diffusible and bound actin nuclei of Xenopus laevis oocytes. Cell, 12: 883891

Conti E, Muller CW, Stewart M (2006) Karyopherin flexibility in nucleocytoplasmic transport. Curr Opin Struct Biol, 16: 237-244

Cook AG, Fukuhara N, Jinek M, Conti E (2009) Structures of the tRNA export factor in the nuclear and cytosolic states. Nature, 461: 60-65

Cooper JA (1987) Effects of cytochalasin and phalloidin on actin. J Cell Biol, 105: 1473-1478

De Meyer T, Muyldermans S, Depicker A (2014) Nanobody-based products as research and diagnostic tools. Trends Biotechnol, 32: 263-270

De Robertis EM, Longthorne RF, Gurdon JB (1978) Intracellular migration of nuclear proteins in Xenopus oocytes. Nature, 272: 254-256

Dean KA, von Ahsen O, Gorlich D, Fried HM (2001) Signal recognition particle protein 19 is imported into the nucleus by importin 8 (RanBP8) and transportin. J Cell Sci, 114: 3479-3485

Denning DP, Patel SS, Uversky V, Fink AL, Rexach M (2003) Disorder in the nuclear pore complex: the FG repeat regions of nucleoporins are natively unfolded. Proc Natl Acad Sci U S A, 100: 2450-2455

Denning DP, Uversky V, Patel SS, Fink AL, Rexach M (2002) The Saccharomyces cerevisiae nucleoporin Nup2p is a natively unfolded protein. $J$ Biol Chem, 277: 33447-33455

Domazetovska A, Ilkovski B, Cooper ST, Ghoddusi M, Hardeman EC, Minamide LS, Gunning PW, Bamburg JR, North KN (2007) Mechanisms underlying intranuclear rod formation. Brain, 130: 3275-3284

Dominguez R (2004) Actin-binding proteins--a unifying hypothesis. Trends Biochem Sci, 29: 572-578

Dominguez R, Holmes KC (2011) Actin structure and function. Annu Rev Biophys, 40: 169-186

Dopie J, Skarp KP, Rajakyla EK, Tanhuanpaa K, Vartiainen MK (2012) Active maintenance of nuclear actin by importin 9 supports transcription. Proc Natl Acad Sci U S A, 109: E544-E552

dos Remedios CG, Chhabra D, Kekic M, Dedova IV, Tsubakihara M, Berry DA, Nosworthy NJ (2003) Actin binding proteins: regulation of cytoskeletal microfilaments. Physiol Rev, 83: 433-473

Erickson HP (2007) Evolution of the cytoskeleton. Bioessays, 29: 668-677

Fawcett DW. (1981) The Cell. W B Saunders Co, 
Feldherr CM (1962) The nuclear annuli as pathways for nucleocytoplasmic exchanges. J Cell Biol, 14: 65-72

Feng JJ, Marston S (2009) Genotype-phenotype correlations in ACTA1 mutations that cause congenital myopathies. Neuromuscul Disord, 19: 6-16

Ferron F, Rebowski G, Lee SH, Dominguez R (2007) Structural basis for the recruitment of profilin-actin complexes during filament elongation by Ena/VASP. EMBO J, 26: 4597-4606

Fischer U, Huber J, Boelens WC, Mattaj IW, Luhrmann R (1995) The HIV-1 Rev activation domain is a nuclear export signal that accesses an export pathway used by specific cellular RNAs. Cell, 82: 475-483

Fomproix N, Percipalle P (2004) An actin-myosin complex on actively transcribing genes. Exp Cell Res, 294: $140-148$

Fornerod M, Ohno M, Yoshida M, Mattaj IW (1997) CRM1 is an export receptor for leucine-rich nuclear export signals. Cell, 90: 1051-1060

Frey S, Gorlich D (2007) A saturated FG-repeat hydrogel can reproduce the permeability properties of nuclear pore complexes. Cell, 130: 512-523

Frey S, Gorlich D (2014) Purification of protein complexes of defined subunit stoichiometry using a set of orthogonal, tag-cleaving proteases. $J$ Chromatogr A, 1337: 106-115

Fukuda M, Asano S, Nakamura T, Adachi M, Yoshida M, Yanagida M, Nishida E (1997) CRM1 is responsible for intracellular transport mediated by the nuclear export signal. Nature, 390: 308-311

Fukui Y, Katsumaru H (1979) Nuclear actin bundles in Amoeba, Dictyostelium and human HeLa cells induced by dimethyl sulfoxide. Exp Cell Res, 120: 451-455

Galkin VE, Orlova A, Salmazo A, Djinovic-Carugo K, Egelman EH (2010) Opening of tandem calponin homology domains regulates their affinity for F-actin. Nat Struct Mol Biol, 17: 614-616

Geerlof A, Brown J, Coutard B, Egloff MP, Enguita FJ, Fogg MJ, Gilbert RJ, Groves MR, Haouz A, Nettleship JE, Nordlund P, Owens RJ, Ruff M, Sainsbury S, Svergun DI, Wilmanns M (2006) The impact of protein characterization in structural proteomics. Acta Crystallogr D Biol Crystallogr, 62: 1125-1136

Gibson DG, Young L, Chuang RY, Venter JC, Hutchison CA, Smith HO (2009) Enzymatic assembly of DNA molecules up to several hundred kilobases. Nat Methods, 6: 343-345

Goebel HH, Warlo I (1997) Nemaline myopathy with intranuclear rods--intranuclear rod myopathy. Neuromuscul Disord, 7: 13-19

Gontan C, Guttler T, Engelen E, Demmers J, Fornerod M, Grosveld FG, Tibboel D, Gorlich D, Poot RA, Rottier RJ (2009) Exportin 4 mediates a novel nuclear import pathway for Sox family transcription factors. $J$ Cell Biol, 185: 27-34

Gordon DJ, Boyer JL, Korn ED (1977) Comparative biochemistry of non-muscle actins. J Biol Chem, 252: $8300-8309$

Gordon DJ, Eisenberg E, Korn ED (1976) Characterization of cytoplasmic actin isolated from Acanthamoeba castellanii by a new method. $J$ Biol Chem, 251: 4778-4786

Gorlich D, Dabrowski M, Bischoff FR, Kutay U, Bork P, Hartmann E, Prehn S, Izaurralde E (1997) A novel class of RanGTP binding proteins. J Cell Biol, 138: $65-80$

Gorlich D, Henklein P, Laskey RA, Hartmann E (1996a) A 41 amino acid motif in importin-alpha confers binding to importin-beta and hence transit into the nucleus. EMBO J, 15: 1810-1817

Gorlich D, Kostka S, Kraft R, Dingwall C, Laskey RA, Hartmann E, Prehn S (1995) Two different subunits of importin cooperate to recognize nuclear localization signals and bind them to the nuclear envelope. Curr Biol, 5: 383-392

Gorlich D, Kutay U (1999) Transport between the cell nucleus and the cytoplasm. Annu Rev Cell Dev Biol, 15: $607-660$ 
Gorlich D, Pante N, Kutay U, Aebi U, Bischoff FR (1996b) Identification of different roles for RanGDP and RanGTP in nuclear protein import. EMBO J, 15: 5584-5594

Gorlich D, Prehn S, Laskey RA, Hartmann E (1994) Isolation of a protein that is essential for the first step of nuclear protein import. Cell, 79: 767-778

Gorlich D, Seewald MJ, Ribbeck K (2003) Characterization of Ran-driven cargo transport and the RanGTPase system by kinetic measurements and computer simulation. EMBO J, 22: 1088-1100

Gorlich D, Vogel F, Mills AD, Hartmann E, Laskey RA (1995) Distinct functions for the two importin subunits in nuclear protein import. Nature, 377: 246-248

Guttler T, Gorlich D (2011) Ran-dependent nuclear export mediators: a structural perspective. EMBO J, 30: $3457-3474$

Guttler T, Madl T, Neumann P, Deichsel D, Corsini L, Monecke T, Ficner R, Sattler M, Gorlich D (2010) NES consensus redefined by structures of PKI-type and Rev-type nuclear export signals bound to CRM1. Nat Struct Mol Biol, 17: 1367-1376

Herman IM (1993) Actin isoforms. Curr Opin Cell Biol, 5: 48-55

Hofmann WA, de Lanerolle P (2006) Nuclear actin: to polymerize or not to polymerize. J Cell Biol, 172: 495-496

Hofmann WA, Stojiljkovic L, Fuchsova B, Vargas GM, Mavrommatis E, Philimonenko V, Kysela K, Goodrich JA, Lessard JL, Hope TJ, Hozak P, de Lanerolle P (2004) Actin is part of pre-initiation complexes and is necessary for transcription by RNA polymerase II. Nat Cell Biol, 6: 1094-1101

Holt MR, Koffer A (2001) Cell motility: proline-rich proteins promote protrusions. Trends Cell Biol, 11: 3846

Hu P, Wu S, Hernandez N (2004) A role for beta-actin in RNA polymerase III transcription. Genes Dev, 18: 3010-3015

Huber J, Cronshagen U, Kadokura M, Marshallsay C, Wada T, Sekine M, Luhrmann R (1998) Snurportin1, an $\mathrm{m} 3 \mathrm{G}$-cap-specific nuclear import receptor with a novel domain structure. EMBO J, 17: 4114-4126

Hutchinson DO, Charlton A, Laing NG, Ilkovski B, North KN (2006) Autosomal dominant nemaline myopathy with intranuclear rods due to mutation of the skeletal muscle ACTA1 gene: clinical and pathological variability within a kindred. Neuromuscul Disord, 16: 113-121

Ilkovski B, Nowak KJ, Domazetovska A, Maxwell AL, Clement S, Davies KE, Laing NG, North KN, Cooper ST (2004) Evidence for a dominant-negative effect in ACTA1 nemaline myopathy caused by abnormal folding, aggregation and altered polymerization of mutant actin isoforms. Hum Mol Genet, 13: $1727-1743$

Iovine MK, Watkins JL, Wente SR (1995) The GLFG repetitive region of the nucleoporin Nup116p interacts with Kap95p, an essential yeast nuclear import factor. J Cell Biol, 131: 1699-1713

Izaurralde E, Kutay U, von Kobbe C, Mattaj IW, Gorlich D (1997) The asymmetric distribution of the constituents of the Ran system is essential for transport into and out of the nucleus. EMBO J, 16: 6535-6547

Jakel S, Albig W, Kutay U, Bischoff FR, Schwamborn K, Doenecke D, Gorlich D (1999) The importin beta/importin 7 heterodimer is a functional nuclear import receptor for histone H1. EMBO J, 18: 2411-2423

Jakel S, Gorlich D (1998) Importin beta, transportin, RanBP5 and RanBP7 mediate nuclear import of ribosomal proteins in mammalian cells. EMBO J, 17: 4491-4502

Jakel S, Mingot JM, Schwarzmaier P, Hartmann E, Gorlich D (2002) Importins fulfil a dual function as nuclear import receptors and cytoplasmic chaperones for exposed basic domains. EMBO J, 21: 377-386

Jullien D, Gorlich D, Laemmli UK, Adachi Y (1999) Nuclear import of RPA in Xenopus egg extracts requires a novel protein XRIPalpha but not importin alpha. EMBO J, 18: 4348-4358 
Kaimaktchiev V, Goebel H, Laing N, Narus M, Weeks D, Nixon R (2006) Intranuclear nemaline rod myopathy. Muscle Nerve, 34: 369-372

Kalderon D, Richardson WD, Markham AF, Smith AE (1984a) Sequence requirements for nuclear location of simian virus 40 large-T antigen. Nature, 311: 33-38

Kalderon D, Roberts BL, Richardson WD, Smith AE (1984b) A short amino acid sequence able to specify nuclear location. Cell, 39: 499-509

Kang H, Bradley MJ, Elam WA, De La Cruz EM (2013) Regulation of actin by ion-linked equilibria. Biophys J, 105: 2621-2628

Kataoka N, Bachorik JL, Dreyfuss G (1999) Transportin-SR, a nuclear import receptor for SR proteins. $J$ Cell Biol, 145: 1145-1152

Kinosian HJ, Selden LA, Gershman LC, Estes JE (2000) Interdependence of profilin, cation, and nucleotide binding to vertebrate non-muscle actin. Biochemistry, 39: 13176-13188

Klebe C, Bischoff FR, Ponstingl H, Wittinghofer A (1995) Interaction of the nuclear GTP-binding protein Ran with its regulatory proteins RCC1 and RanGAP1. Biochemistry, 34: 639-647

Korotkov KV, Pardon E, Steyaert J, Hol WG (2009) Crystal structure of the N-terminal domain of the secretin GspD from ETEC determined with the assistance of a nanobody. Structure, 17: 255-265

Kurisaki A, Kurisaki K, Kowanetz M, Sugino H, Yoneda Y, Heldin CH, Moustakas A (2006) The mechanism of nuclear export of Smad3 involves exportin 4 and Ran. Mol Cell Biol, 26: 1318-1332

Kutay U, Bischoff FR, Kostka S, Kraft R, Gorlich D (1997) Export of importin alpha from the nucleus is mediated by a specific nuclear transport factor. Cell, 90: 1061-1071

Kutay U, Lipowsky G, Izaurralde E, Bischoff FR, Schwarzmaier P, Hartmann E, Gorlich D (1998) Identification of a tRNA-specific nuclear export receptor. Mol Cell, 1: 359-369

Laing NG, Dye DE, Wallgren-Pettersson C, Richard G, Monnier N, Lillis S, Winder TL, Lochmuller H, Graziano C, Mitrani-Rosenbaum S, Twomey D, Sparrow JC, Beggs AH, Nowak KJ (2009) Mutations and polymorphisms of the skeletal muscle alpha-actin gene (ACTA1). Hum Mutat, 30: 1267-1277

Lam AY, Pardon E, Korotkov KV, Hol WG, Steyaert J (2009) Nanobody-aided structure determination of the EpsI:EpsJ pseudopilin heterodimer from Vibrio vulnificus. $J$ Struct Biol, 166: 8-15

Larsson H, Lindberg U (1988) The effect of divalent cations on the interaction between calf spleen profilin and different actins. Biochim Biophys Acta, 953: 95-105

Lassing I, Lindberg U (1985) Specific interaction between phosphatidylinositol 4,5-bisphosphate and profilactin. Nature, 314: 472-474

Lee SJ, Sekimoto T, Yamashita E, Nagoshi E, Nakagawa A, Imamoto N, Yoshimura M, Sakai H, Chong KT, Tsukihara T, Yoneda Y (2003) The structure of importin-beta bound to SREBP-2: nuclear import of a transcription factor. Science, 302: 1571-1575

Lindberg U (1967) Purification from calf spleen of two inhibitors of deoxyribonuclease. I. Physical and chemical characterization of the inhibitor II. Biochemistry, 6: 323-335

Lipowsky G, Bischoff FR, Schwarzmaier P, Kraft R, Kostka S, Hartmann E, Kutay U, Gorlich D (2000) Exportin 4: a mediator of a novel nuclear export pathway in higher eukaryotes. EMBO J, 19: 4362-4371

Loris R, Marianovsky I, Lah J, Laeremans T, Engelberg-Kulka H, Glaser G, Muyldermans S, Wyns L (2003) Crystal structure of the intrinsically flexible addiction antidote MazE. J Biol Chem, 278: 28252-28257

Mahajan R, Delphin C, Guan T, Gerace L, Melchior F (1997) A small ubiquitin-related polypeptide involved in targeting RanGAP1 to nuclear pore complex protein RanBP2. Cell, 88: 97-107

Matsuura Y, Stewart M (2004) Structural basis for the assembly of a nuclear export complex. Nature, 432: 872-877 
Matunis MJ, Coutavas E, Blobel G (1996) A novel ubiquitin-like modification modulates the partitioning of the Ran-GTPase-activating protein RanGAP1 between the cytosol and the nuclear pore complex. J Cell Biol, 135: $1457-1470$

Mayeda A, Krainer AR (1999) Preparation of HeLa cell nuclear and cytosolic S100 extracts for in vitro splicing. Methods Mol Biol, 118: 309-314

McDonald D, Carrero G, Andrin C, de Vries G, Hendzel MJ (2006) Nucleoplasmic beta-actin exists in a dynamic equilibrium between low-mobility polymeric species and rapidly diffusing populations. J Cell Biol, 172: $541-552$

Mingot JM, Bohnsack MT, Jakle U, Gorlich D (2004) Exportin 7 defines a novel general nuclear export pathway. EMBO J, 23: 3227-3236

Mingot JM, Kostka S, Kraft R, Hartmann E, Gorlich D (2001) Importin 13: a novel mediator of nuclear import and export. EMBO J, 20: 3685-3694

Mitrousis G, Olia AS, Walker-Kopp N, Cingolani G (2008) Molecular basis for the recognition of snurportin 1 by importin beta. J Biol Chem, 283: 7877-7884

Mockrin SC, Korn ED (1980) Acanthamoeba profilin interacts with G-actin to increase the rate of exchange of actin-bound adenosine 5'-triphosphate. Biochemistry, 19: 5359-5362

Mogridge J (2004) Using light scattering to determine the stoichiometry of protein complexes. Methods Mol Biol, 261: 113-118

Mohr D, Frey S, Fischer T, Guttler T, Gorlich D (2009) Characterisation of the passive permeability barrier of nuclear pore complexes. EMBO J, 28: 2541-2553

Monecke T, Guttler T, Neumann P, Dickmanns A, Gorlich D, Ficner R (2009) Crystal structure of the nuclear export receptor CRM1 in complex with Snurportin1 and RanGTP. Science, 324: 1087-1091

Monecke T, Haselbach D, Voss B, Russek A, Neumann P, Thomson E, Hurt E, Zachariae U, Stark H, Grubmuller H, Dickmanns A, Ficner R (2013) Structural basis for cooperativity of CRM1 export complex formation. Proc Natl Acad Sci U S A, 110: 960-965

Moore MS, Blobel G (1994) Purification of a Ran-interacting protein that is required for protein import into the nucleus. Proc Natl Acad Sci U S A, 91: 10212-10216

Morton WM, Ayscough KR, McLaughlin PJ (2000) Latrunculin alters the actin-monomer subunit interface to prevent polymerization. Nat Cell Biol, 2: 376-378

Muhlhausser P, Muller EC, Otto A, Kutay U (2001) Multiple pathways contribute to nuclear import of core histones. EMBO Rep, 2: 690-696

Muyldermans S (2013) Nanobodies: natural single-domain antibodies. Annu Rev Biochem, 82: 775-797

Nowak KJ, Ravenscroft G, Laing NG (2013) Skeletal muscle alpha-actin diseases (actinopathies): pathology and mechanisms. Acta Neuropathol, 125: 19-32

Ohnishi T, Kawamura H, Tanaka Y (1964) Actin and myosin-like proteins in the calf thymus cell nucleus. $J$ Biochem, 56: 6-15

Ohshima S, Abe H, Obinata T (1989) Isolation of profilin from embryonic chicken skeletal muscle and evaluation of its interaction with different actin isoforms. J Biochem, 105: 855-857

Ori A, Banterle N, Iskar M, Andres-Pons A, Escher C, Khanh Bui H, Sparks L, Solis-Mezarino V, Rinner O, Bork P, Lemke EA, Beck M (2013) Cell type-specific nuclear pores: a case in point for context-dependent stoichiometry of molecular machines. Mol Syst Biol, 9: 648

Osborn M, Weber K (1980) Dimethylsulfoxide and the ionophore A23187 affect the arrangement of actin and induce nuclear actin paracrystals in PtK2 cells. Exp Cell Res, 129: 103-114

Paraskeva E, Izaurralde E, Bischoff FR, Huber J, Kutay U, Hartmann E, Luhrmann R, Gorlich D (1999) CRM1-mediated recycling of snurportin 1 to the cytoplasm. J Cell Biol, 145: 255-264 
Pardee JD, Spudich JA (1982) Purification of muscle actin. Methods Enzymol, 85 Pt B: 164-181

Park SH, Park TJ, Lim IK (2011) Reduction of exportin 6 activity leads to actin accumulation via failure of RanGTP restoration and NTF2 sequestration in the nuclei of senescent cells. Exp Cell Res, 317: 941-954

Partridge JR, Schwartz TU (2009) Crystallographic and biochemical analysis of the Ran-binding zinc finger domain. J Mol Biol, 391: 375-389

Perrimon N, Engstrom L, Mahowald AP (1989) Zygotic lethals with specific maternal effect phenotypes in Drosophila melanogaster. I. Loci on the X chromosome. Genetics, 121: 333-352

Perrin BJ, Ervasti JM (2010) The actin gene family: function follows isoform. Cytoskeleton (Hoboken), 67: $630-634$

Philimonenko VV, Zhao J, Iben S, Dingova H, Kysela K, Kahle M, Zentgraf H, Hofmann WA, de Lanerolle P, Hozak P, Grummt I (2004) Nuclear actin and myosin I are required for RNA polymerase I transcription. Nat Cell Biol, 6: 1165-1172

Plafker SM, Macara IG (2000) Importin-11, a nuclear import receptor for the ubiquitin-conjugating enzyme, UbcM2. EMBO J, 19: 5502-5513

Plafker SM, Macara IG (2002) Ribosomal protein L12 uses a distinct nuclear import pathway mediated by importin 11. Mol Cell Biol, 22: 1266-1275

Pollard TD (1986) Assembly and dynamics of the actin filament system in nonmuscle cells. $J$ Cell Biochem, 31: $87-95$

Pollard TD, Borisy GG (2003) Cellular motility driven by assembly and disassembly of actin filaments. Cell, 112: $453-465$

Pollard TD, Cooper JA (1986) Actin and actin-binding proteins. A critical evaluation of mechanisms and functions. Annu Rev Biochem, 55: 987-1035

Pollard TD, Cooper JA (2009) Actin, a central player in cell shape and movement. Science, 326: 1208-1212

Pollard VW, Michael WM, Nakielny S, Siomi MC, Wang F, Dreyfuss G (1996) A novel receptor-mediated nuclear protein import pathway. Cell, 86: 985-994

Ravenscroft G, Wilmshurst JM, Pillay K, Sivadorai P, Wallefeld W, Nowak KJ, Laing NG (2011) A novel ACTA1 mutation resulting in a severe congenital myopathy with nemaline bodies, intranuclear rods and type I fibre predominance. Neuromuscul Disord, 21: 31-36

Reichelt R, Holzenburg A, Buhle ELJ, Jarnik M, Engel A, Aebi U (1990) Correlation between structure and mass distribution of the nuclear pore complex and of distinct pore complex components. J Cell Biol, 110: 883-894

Reichstein E, Korn ED (1979) Acanthamoeba profilin. A protein of low molecular weight from Acanpthamoeba castellanii that inhibits actin nucleation. J Biol Chem, 254: 6174-6179

Ribbeck K, Gorlich D (2001) Kinetic analysis of translocation through nuclear pore complexes. EMBO J, 20: $1320-1330$

Ribbeck K, Gorlich D (2002) The permeability barrier of nuclear pore complexes appears to operate via hydrophobic exclusion. EMBO J, 21: 2664-2671

Ribbeck K, Lipowsky G, Kent HM, Stewart M, Gorlich D (1998) NTF2 mediates nuclear import of Ran. EMBO J, 17: 6587-6598

Richards SA, Lounsbury KM, Carey KL, Macara IG (1996) A nuclear export signal is essential for the cytosolic localization of the Ran binding protein, RanBP1. J Cell Biol, 134: 1157-1168

Richards SA, Lounsbury KM, Macara IG (1995) The C terminus of the nuclear RAN/TC4 GTPase stabilizes the GDP-bound state and mediates interactions with RCC1, RAN-GAP, and HTF9A/RANBP1. J Biol Chem, 270: $14405-14411$ 
Robbins J, Dilworth SM, Laskey RA, Dingwall C (1991) Two interdependent basic domains in nucleoplasmin nuclear targeting sequence: identification of a class of bipartite nuclear targeting sequence. Cell, 64: 615-623

Roeder AD, Gard DL (1994) Confocal microscopy of F-actin distribution in Xenopus oocytes. Zygote, 2: $111-124$

Rohn JL, Sims D, Liu T, Fedorova M, Schock F, Dopie J, Vartiainen MK, Kiger AA, Perrimon N, Baum B (2011) Comparative RNAi screening identifies a conserved core metazoan actinome by phenotype. $J$ Cell Biol, 194: 789-805

Rout MP, Blobel G (1993) Isolation of the yeast nuclear pore complex. J Cell Biol, 123: 771-783

Safer D, Elzinga M, Nachmias VT (1991) Thymosin beta 4 and Fx, an actin-sequestering peptide, are indistinguishable. J Biol Chem, 266: 4029-4032

Sambrook J. (2001) Molecular Cloning: A Laboratory Manual, Third Edition (3 volume set). Cold Spring Harbor Laboratory Press,

Samwer M, Dehne HJ, Spira F, Kollmar M, Gerlich DW, Urlaub H, Gorlich D (2013) The nuclear F-actin interactome of Xenopus oocytes reveals an actin-bundling kinesin that is essential for meiotic cytokinesis. EMBO J, 32: 1886-1902

Sanger JW, Sanger JM, Kreis TE, Jockusch BM (1980) Reversible translocation of cytoplasmic actin into the nucleus caused by dimethyl sulfoxide. Proc Natl Acad Sci U S A, 77: 5268-5272

Seewald MJ, Korner C, Wittinghofer A, Vetter IR (2002) RanGAP mediates GTP hydrolysis without an arginine finger. Nature, 415: 662-666

Segura M, Lindberg U (1984) Separation of non-muscle isoactins in the free form or as profilactin complexes. J Biol Chem, 259: 3949-3954

Shaevitz JW, Gitai Z (2010) The structure and function of bacterial actin homologs. Cold Spring Harb Perspect Biol, 2: a000364

Sjolinder M, Bjork P, Soderberg E, Sabri N, Farrants AK, Visa N (2005) The growing pre-mRNA recruits actin and chromatin-modifying factors to transcriptionally active genes. Genes Dev, 19: 1871-1884

Sohn RH, Goldschmidt-Clermont PJ (1994) Profilin: at the crossroads of signal transduction and the actin cytoskeleton. Bioessays, 16: 465-472

Sparrow JC, Nowak KJ, Durling HJ, Beggs AH, Wallgren-Pettersson C, Romero N, Nonaka I, Laing NG (2003) Muscle disease caused by mutations in the skeletal muscle alpha-actin gene (ACTA1). Neuromuscul Disord, 13: 519-531

Spudich JA, Watt S (1971) The regulation of rabbit skeletal muscle contraction. I. Biochemical studies of the interaction of the tropomyosin-troponin complex with actin and the proteolytic fragments of myosin. J Biol Chem, 246: 4866-4871

Stuven T, Hartmann E, Gorlich D (2003) Exportin 6: a novel nuclear export receptor that is specific for profilin.actin complexes. EMBO J, 22: 5928-5940

Subramanian K, Meyer T (1997) Calcium-induced restructuring of nuclear envelope and endoplasmic reticulum calcium stores. Cell, 89: 963-971

Tanaka M, Shibata H (1985) Poly(L-proline)-binding proteins from chick embryos are a profilin and a profilactin. Eur J Biochem, 151: 291-297

Tilney LG, Bonder EM, Coluccio LM, Mooseker MS (1983) Actin from Thyone sperm assembles on only one end of an actin filament: a behavior regulated by profilin. J Cell Biol, 97: 112-124

Tobacman LS, Korn ED (1982) The regulation of actin polymerization and the inhibition of monomeric actin ATPase activity by Acanthamoeba profilin. J Biol Chem, 257: 4166-4170 
Trotta CR, Lund E, Kahan L, Johnson AW, Dahlberg JE (2003) Coordinated nuclear export of 60S ribosomal subunits and NMD3 in vertebrates. EMBO J, 22: 2841-2851

Truant R, Cullen BR (1999) The arginine-rich domains present in human immunodeficiency virus type 1 Tat and Rev function as direct importin beta-dependent nuclear localization signals. Mol Cell Biol, 19: 12101217

Vartiainen MK, Guettler S, Larijani B, Treisman R (2007) Nuclear actin regulates dynamic subcellular localization and activity of the SRF cofactor MAL. Science, 316: 1749-1752

Vetter IR, Arndt A, Kutay U, Gorlich D, Wittinghofer A (1999) Structural view of the Ran-Importin beta interaction at 2.3 A resolution. Cell, 97: 635-646

Vinson VK, De La Cruz EM, Higgs HN, Pollard TD (1998) Interactions of Acanthamoeba profilin with actin and nucleotides bound to actin. Biochemistry, 37: 10871-10880

Wada A, Fukuda M, Mishima M, Nishida E (1998) Nuclear export of actin: a novel mechanism regulating the subcellular localization of a major cytoskeletal protein. EMBO J, 17: 1635-1641

Watson ML (1954) Pores in the mammalian nuclear membrane. Biochim Biophys Acta, 15: 475-479

Watson ML (1955) The nuclear envelope; its structure and relation to cytoplasmic membranes. J Biophys Biochem Cytol, 1: 257-270

Watson ML (1959) Further observations on the nuclear envelope of the animal cell. J Biophys Biochem Cytol, 6: $147-156$

Wegner A (1982) Treadmilling of actin at physiological salt concentrations. An analysis of the critical concentrations of actin filaments. J Mol Biol, 161: 607-615

Weinmann L, Hock J, Ivacevic T, Ohrt T, Mutze J, Schwille P, Kremmer E, Benes V, Urlaub H, Meister G (2009) Importin 8 is a gene silencing factor that targets argonaute proteins to distinct mRNAs. Cell, 136: 496-507

Wen W, Harootunian AT, Adams SR, Feramisco J, Tsien RY, Meinkoth JL, Taylor SS (1994) Heat-stable inhibitors of cAMP-dependent protein kinase carry a nuclear export signal. J Biol Chem, 269: 32214-32220

Wen W, Meinkoth JL, Tsien RY, Taylor SS (1995) Identification of a signal for rapid export of proteins from the nucleus. Cell, 82: 463-473

Wulf E, Deboben A, Bautz FA, Faulstich H, Wieland T (1979) Fluorescent phallotoxin, a tool for the visualization of cellular actin. Proc Natl Acad Sci U S A, 76: 4498-4502

Wyatt PJ (1993) Light scattering and the absolute characterization of macromolecules. Analytica chimica acta, 272: $1-40$

Yang Q, Rout MP, Akey CW (1998) Three-dimensional architecture of the isolated yeast nuclear pore complex: functional and evolutionary implications. Mol Cell, 1: 223-234

Yokoyama N, Hayashi N, Seki T, Pante N, Ohba T, Nishii K, Kuma K, Hayashida T, Miyata T, Aebi U, et A (1995) A giant nucleopore protein that binds Ran/TC4. Nature, 376: 184-188

Yoshida K, Blobel G (2001) The karyopherin Kap142p/Msn5p mediates nuclear import and nuclear export of different cargo proteins. J Cell Biol, 152: 729-740

Zhao K, Wang W, Rando OJ, Xue Y, Swiderek K, Kuo A, Crabtree GR (1998) Rapid and phosphoinositoldependent binding of the SWI/SNF-like BAF complex to chromatin after T lymphocyte receptor signaling. Cell, 95: 625-636 


\section{Curriculum vitae}

$\begin{array}{ll}\text { Name } & \text { Kevser Gencalp } \\ \text { Date of birth } & \mathbf{1 7 . 0 4 . 1 9 8 6} \\ \text { Place of birth } & \text { Izmir / Turkey } \\ \text { Nationality } & \text { Turkish }\end{array}$

\section{Education}

$2010-2014$

$2009-2010$

MSc in Molecular Biology

Max Planck Institute for Biophysical Chemistry, Göttingen, Germany Department of cellular Logistics

International Max Planck Research School, Molecular Biology

2004- 2009

BSc in Molecular Biology and Genetics

Middle East Technical University, Ankara, Turkey

$1997-\mathbf{2 0 0 4}$

Bornova Anadolu Lisesi (High School), Izmir / Turkey 